\title{
NMR STRUCTURAL STUDIES ON THE PERIPLASMIC DOMAIN OF CitA and DcuS
}

\author{
Dissertation \\ zur Erlangung des Doktorgrades \\ der Mathematisch-Naturwissenschaftlichen Fakultäten \\ der Georg-August-Universität zu Göttingen
}

\author{
vorgelegt von \\ Vinesh Vijayan \\ aus VAIKOM, INDIA
}

Göttingen 2007 
D7

Referent: Prof. Dr. Christian Griesinger

Korreferent: Prof. Dr. Martin Suhm

Tag der mündlichen Prüfung: 


\section{Publication lists}

This thesis is based on the following papers:

Chapter 3

\{1\} V. Vijayan and M. Zweckstetter, Simultaneous measurement of protein one-bond residual dipolar couplings without increased resonance overlap, Journal of Magnetic Resonance, 174(2): 245-253, 2005.

Chapter 4

$\{2\}$ L. Pappalardo, and I.G. Janausch, V. Vijayan, E. Zientz, J. Junker, W. Peti, M. Zweckstetter, G. Unden, and C. Griesinger, The NMR structure of the sensory domain of the membranous Two-Component fumarate sensor (histidine protein kinase) Dcus of Escherichia Coli, Journal of biological chemistry, 278(40): 39185-38188, 2003.

$\{3\}$ H. Kneuper, I.J. Janausch, V. Vijayan, M. Zweckstetter, V. Bock, C. Griesinger, and G. Unden, The nature of the stimulus and of the fumarate binding site of the fumarate sensor Dcus of Escherichia Coli, Journal of biological chemistry, 280(21): 20596-20603, 2005.

\{4\} M. Sevvana, V. Vijayan, M. Zweckstetter, S. Reinelt, D.R. Madden, R. Herbst-Immer, G.M. Sheldrick, M. Bott, C. Griesinger and S. Becker, Ligand-induced switch mechanism regulates signal transduction in sensor histidine kinase CitA, submitted, 2007.

\section{Related papers :}

\{5\} S. Rumpel, A. Razeto, C. M. Pillar, V. Vijayan, A. Taylor, K. Giller, M.S. Gilmore, S. Becker, and M. Zweckstetter, Structure and DNA-binding properties of the cytolysin regulator Cylr2 from Enterococcus Faecalis, EMBO journal, 23(18): 3632-3642, 2004. 
\{6\} M. Bayrhuber, V. Vijayan, M. Ferber, R. Graf, J. Korukottu, J. Imperial, J.E. Garrett, B.M. Olivera, H. Terlau, M. Zweckstetter, and S. Becker, Conkunitzin-S1 ss the first member of a new Kunitz-type neurotoxin family. Structural and functional characterization, Journal of biological chemistry, 280(25) : 23766-23770, 2005.

\{7\} S. Rumpel, H.Y. Kim, V.Vijayan, S. Becker, and M. Zweckstetter, Backbone resonance assignment of the homodimeric, $35 \mathrm{Kda}$ chaperone Cest from enteropathogenic Escherichia Coli, Journal of Biomolecular NMR, 31(4) : 377-378, 2005.

$\{8\}$ P. Montaville, H.Y. Kim, V. Vijayan, S. Becker, and M. Zweckstetter, ${ }^{1} \mathrm{H}^{N}$, ${ }^{15} \mathrm{~N}$, and ${ }^{13} \mathrm{C}$ Resonance assignment of the C2a Domain of Rabphilin3a, Journal of Biomolecular NMR, 36(5) : 20, 2006.

\{9\} J. Korukottu, M. Bayrhuber, P. Montaville, V. Vijayan, Y.S. Jung, S. Becker, M. Zweckstetter, Fast high-resolution protein structure determination by using unassigned NMR data, Angewandte Chemie International Edition English, 46(7) : 1176-1179, 2007.

$\{10\}$ J. Korukottu, A. Lange, V. Vijayan, R. Schneider, O. Pongs, S Becker, M. Baldus, and M. Zweckstetter, Conformational plasticity in ion channel recognition of a peptide toxin. to be communicated, 2007. 


\section{Acknowledgments}

Now that I have reached the end of a long journey in crafting this thesis, its time to express my gratitude to all those who have been a part of this travel. I have constantly wondered as to how I would feel at this exact moment ever since I landed in Germany in the fall of 2002 (I was then naive, full of hope, and optimistic). A rather quick four and half years later, as I write this, I am filled with mixed emotions. In retrospect, the last four and a half years in my life has been credible in that it has been intellectually satisfying. Thanks to all the help I received. On the other hand, I would have been furthermore at ease if I had been little more systematic. It should have definitely helped me overcome the sleepless nights I have had owing to the incredible stress.

I express my profound gratitude to Prof. Christian Griesinger, for his excellent guidance and support throughout the course of my thesis. The discussions we had, were immensely stimulating and thought provoking. I am indebted to him for the affection he showered on me and it was a great pleasure to work with him.

I would like to thank Dr. Markus Zweckstetter, my group leader, for his help with many of my projects, for his immense faith and confidence in me, in spite of all my slip-ups.

I thank my collaborators, Prof. Gotfried Unden, Prof. Micheal Bott and their group,in DcuS and CitA projects. I owe my thanks to Dr. Stefan Becker of our molecular biology lab for his outstanding guidance in preparing excellent protein samples for my NMR measurements. A thanks doesn't seem sufficient to Dr. Stefan Becker and Karin Giller for the stable CitAP protein they produced. But it is said with lots of appreciation.

The road to my graduation has been long and winding. There are quite a list of people with whom I have had the privilege to work during this 4.5 years. I am grateful to all the 
present and former members of our group, who have contributed in every minute way to make my stay in the group a very memorable one. Mere words are insufficient to express my thanks to Pierre Montaville, who is my trusted friend, my counselor and a brotherly figure, all rolled into one. It was a pleasure working with him throughout the entire tenure of my Ph.D. It was through his great assistance that I managed to overcome my inhibition for biological systems. A special thanks to Pierre for having taken a close look at both the english grammar and scientific content, correcting both and offering suggestions for improvement. Many thanks to Jung Sang Jung. He made my first three years of stay in Göttingen a pleasant and memorable one. The valuable time he spent with me for long chocolate and coffee breaks especially during the weekends will always be treasured.

During my entire stay, I was fortunate enough to share my work space with wonderful people: Dirk Lennartz, my first room mate. If not for his extended support and help, getting myself adapted to German environment would have been more difficult. Sigrun Rumpel, for her timely coffee breaks that drove away my boredom. Monika Bayrhuber, along with Sigrun for the innumerable translations they patiently did. Daniela Fischer and Julien Orts for their pleasant company. Many thanks to all of them. I had the great pleasure of working together with them in few of their projects and I appreciate their feed backs in my projects as well.

And then there are all the people who have made Göttingen a very special place over this four years. Nils, Marco, Fernando, Kerstin, Minkyu, Hai-Young, Pinar, Dirk Bockelmann, Christophe, Nicolas, Jochen, Devan, Raghav, Jegannath, Peter, Valerie, Lukasz, Karel, Jörg, Edith, Melanie and the rest of my friends in our group. I acknowledge their friendly affection, especially Nils, Marco, Fernando, Kerstin, Minkyu and Hai-Young, for lending a patient ear to all my problems and for their warm support all along the way. Thanks to Dr. Donghan Lee for his interesting discussions on NMR and other topics. I also like to specifically thank, Dirk Bockelmann, Christophe, Nils, Minkyu and Lukasz for their kind help in spectrometer maintenance.

My sincere thanks to Mrs. Silberer, secretary to Prof. Griesinger for her ever helping attitude in dealing with bureaucratic and other paper works. 
I am indebted to Prof. Dr. Martin Suhm for being the Korreferent of this thesis work. In addition I would like to thank Prof Grubmüller for kindly accepting the examiner role in my Rigorosum examination. I would also like to thank Prof. Diederichsen, Prof. Buback and Prof. Sheldrick for their kind consent to be a member of my Prüfungskommission.

I sincerely thank Dr. Thomas Jovin and Dr. Donna Arndt-Jovin for the engaging discussions, we have had, although few in numbers. I gratefully acknowledge Dr. Reinhard Klement at the Department of Molecular biology, for his well-timed help in fixing my computer, when it failed to cooperate just a week before the D-day. Many heartfelt thanks to Prof. Elizabeth Jares-Erijman. It was through her that my wife and I got introduced to the delicious world of "Dulce de Leche". Eversince, I have been an addict to it, which I don't regret.

My heartfelt thanks to all my buddies outside NMR department Rebecca, Harshad, Guy and especially to my Argentinean "amigos dulces" Fito, Valeria, Carlos, Marianela, Claudio, Sole C, Sole G for the copious supplies of Dulce de Leche they provided.

I would like to thank my cousin Nisanth for his encouragement during my PhD, Shankaragayathri for her timely help, particularly during the drafting of my thesis. A special word of thanks to my junior C.Subbu, for the unconditional love he showers on me, and whom I consider as my younger brother.

I dedicate this thesis to my father and my mother whose unlimited love, patience has seen me through all the frustrations of being alone during the first half of my $\mathrm{PhD}$ tenure. I thank my sister Veena and her husband Shyam for their understanding, support and affection. Last but not least, my wife Shyamala, love of my life. Without her, I would not be where I am today. 


\section{Zusammenfassung}

Zwei-Komponenten regulatorische Systeme sind die häufigsten Systeme für transmembrane Signaltransduktion in Bakterien und spielen eine Hauptrolle bei der zellulären Adaptation an die Bedingungen der Umwelt und Stress. Sie bestehen aus zwei verschiedenen Proteinen, einer sensorischen Histidinkinase, die normalerweise in der Membran lokalisiert ist, und einem verwandten Antwortregulator im Cytoplasma. Für diese Systeme gibt es eine Fülle von molekularbiologischen Studien. Trotzdem sind keine Strukturinformationen über die transmembrane Signaltransduktion vorhanden. Das Ziel dieser Untersuchung war es, Informationen über die Struktur und Dynamik der Signalerkennung und -transduktion von der periplasmatischen sensorischen Domäne über den membranständigen zwei-Komponenten Sensor in die cytoplasmatische Domäne zu erhalten. In dieser Arbeit werden Strukturuntersuchungen mittels NMR an der periplasmatischen Domäne von zwei Histidinkinasen präsentiert.

DcuS und CitA sind bakterielle Sensorhistidinkinasen, die eine transmembrane Domäne besitzen. Sie sind Teil eines zwei-Komponenten Signaltransduktionssystems, das den Transport und Metabolismus von Di- und Tri-Carboxylaten in Abhängigkeit ihrer Konzentration in der Umgebung regulieren. Ihre periplasmitischen Domänen (DcuS-PD und CitAP) sind homolog, haben eine PAS-Domäne und eine Bindungsstelle für die Carboxylate. CitA fungiert als ein hochspezifischer Citratrezeptor während DcuS von einer Reihe von C4Dicarboxylaten wie Fumarat und Succinat stimuliert wird. Als ein erster Schritt in Hinblick auf die Aufklärung des Signaltransduktionsprozesses wurde die Lösungs-NMR-Struktur der periplasmatischen Domäne von DcuS gelöst. Die Struktur wurde mit residualen dipolaren Kopplungen (RDCs), die über eine neuartige Strategie zur simultanen Messung von RDCs mit minimalem Resonanzüberlap gemessen wurden, verfeinert. Die Bindungstasche von DcuS-PD für einige C4 Di-Carboxylate wurde mittels ${ }^{15} \mathrm{~N}-{ }^{1} \mathrm{H}$ HSQC basierter Titrationen definiert. Der Einfluss der Ligandenbindung an DcuS-PD war schwach. Weder Veränderungen 
der chemischen Verschiebungen noch Anstieg der Signalintensitäten für Reste außerhalb der Bindungstasche wurden beobachtet. Deshalb blieb der Mechanismus der Signalransduktion ungewiss.

Lösungs-NMR-Strukturen von CitAP konnten aufgrund starker Linienverbreiterung, die in den NMR-Spektren beobachtet wurde, nicht gelöst werden. Konformationeller Austausch war der Hauptgrund der Linienverbreiterung. Die Kristallstrukturen der citrat-freien und -gebundenen Form von CitAP konnten aufgeklärt werden. Hauptunterschiede wurden in der Citratbindungsregion und in der C-terminalen Region des Proteins beobachtet. Zusätzlich veränderten sich die chemischen Verschiebungen und die HetNOE-Werte in diesen Teilen des Proteins stark. In der citrat-gebundenen Struktur wurde ein $\mathrm{Na}^{+}$-Ion zwischen die Nterminale Helix und die $\beta$-Faltblätter gesetzt. Das wurde auch durch NMR-Titrationen bestätigt. Damit könnte CitAP in Lösung sowohl an der Erkennung von Citrat als auch von $\mathrm{Na}^{+}$beteiligt sein. Überraschenderweise passen die für cirtatfreies CitAP gemessenen RDCs besser zu der citratgebundenen Struktur von CitAP. Das deutet darauf hin, dass in Lösung eine vorgeformte Bindungstasche von CitAP vorliegt. Nichtsdestotrotz ermöglichten die spezifische strukturellen Unterschiede zwischen der citratfreien und den -gebundenen Strukturen den Vorschlag eines Modells für den Mechanismus der Signaltransduktion. Dieses Modell passt zu den verfügbaren NMR-Daten und ist auch ähnlich zu dem für Aspartatsensoren beschriebenen Mechanismus der Signaltransduktion . 


\section{Abstract}

Two-component regulatory system represent the most frequent system for transmembrane signaling in bacteria and play a major role in the cellular adaptation to environmental conditions and stress. They consist of two separate proteins, a sensory histidine protein kinase which is located typically in the membrane, and a cognate response regulator in the cytoplasm. Despite the wealth of molecular biological studies in these systems, no structural informations are available on the signal transduction by these systems across the membrane. The aim of the study was to gain structural and dynamic information on signal perception and signal transduction from the periplasmic sensor domain of the two component membraneous sensor into the cytoplasmic domain. In this thesis NMR structural studies on the periplasmic domain of two histidine kinase are presented.

DcuS and CitA are bacterial membraneous sensory histidine kinases. They are part of a two component signal transduction systems that regulate the transport and metabolism of di- and tri-carboxylates in response to their environmental concentration. Their periplasmic domains (DcuS-PD and CitAP), are homologous, share a PAS fold, and contain the binding site for the carboxylates. CitA works as a highly specific citrate receptor whereas DcuS uses a wider range of $\mathrm{C} 4$ dicarboxylates like fumarate, succinate etc as stimulus. As a first step to understand the signal transfer process, the NMR solution structure of periplasmic domain of DcuS was determined. The structure was refined with residual dipolar couplings (RDCs), measured using a novel strategy for simultaneous measurement of RDCs with minimum resonance overlap. The binding pocket of DcuS-PD for C4 di-carboxylates was defined using ${ }^{15} \mathrm{~N}-{ }^{1} \mathrm{H}$ HSQC based titrations. The effect of the ligand binding to DcuS-PD was weak. No chemical shift changes or intensity increase for residues were observed outside the binding pocket and hence the signal transduction mechanism remained undetermined. Therefore the 
sensory domain of CitAP which binds citrate more specifically was studied to obtain a better understanding of the conformational changes that lead to signal transduction.

The NMR solution structures of CitAP could not be determined because of the large number of missing peaks due to severe line broadening observed in the NMR spectra. Conformational exchange was the major cause of line broadening. However the X-ray structures of citrate free and bound form of CitAP could be determined. The major conformational changes were observed in the citrate binding region and in the C-terminal region of the protein. Large chemical shift changes and Het-NOE values were also observed in these parts of the protein. In the citrate bound structure, a $\mathrm{Na}^{+}$ion was tentatively localized between $\mathrm{N}$ terminal helix and the $\beta$ sheets. This was also confirmed by NMR titrations. Hence CitAP may be involved in sensing both citrate and $\mathrm{Na}^{+}$ion in solution. Surprisingly the RDCs measured for citrate free CitAP fit better with citrate bound structure of CitAP. This indicates a pre-formed binding pocket of CitAP in solution. Nevertheless, the specific structural differences between the citrate free and bound structures allowed to formulate a model for the mechanism of signal transduction. This model is consistent with available NMR data and also very similar to the signal transduction mechanism described for aspartate sensors. 


\section{Table of Contents}

Page

Publication lists ..................... i i

Zusammenfassung ...................... vii

Abstract ....................... ix

List of Abbreviations . . . . . . . . . . . . . . . . xxi

CHAPTER

1 Introduction . . . . . . . . . . . . . . . 1

1.1 General Introduction . . . . . . . . . . . . . . . . . . . . . 1

1.2 Backbone chemical shift assignment . . . . . . . . . . . . . . . . . . . 2

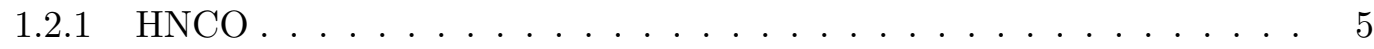

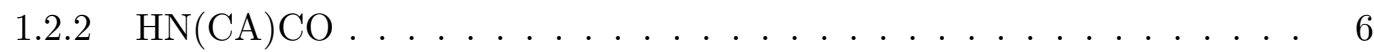

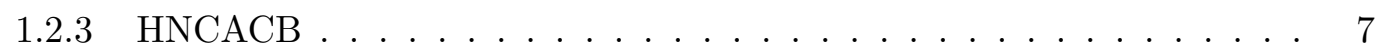

$1.2 .4 \mathrm{CBCA}(\mathrm{CO}) \mathrm{NH} \ldots \ldots \ldots \ldots \ldots$

$1.2 .5 \quad$ Assignment strategy $\ldots \ldots \ldots \ldots \ldots$

1.3 Residual Dipolar Couplings (RDC) . . . . . . . . . . . . . . . . . 9

$1.3 .1 \quad$ Alignment tensor determination for a rigid molecule . . . . . . . . . . 11

1.3 .2 Measurement of the RDCs . . . . . . . . . . . . . . . 14

2 Materials and Methods ............... 15

2.1 Materials . . . . . . . . . . . . . . . . . . . . . . . . . . . . . . . . . 15

$2.1 .1 \quad$ Ubiquitin . . . . . . . . . . . . . . . . . . . . 15

$2.1 .2 \quad$ Periplasmic domain of DcuS (DcuS-PD) . . . . . . . . . . . 15

$2.1 .3 \quad$ Periplasmic domain of CitA (CitAP) $\ldots \ldots \ldots \ldots$ 
2.1 .4 Equipment $\ldots \ldots \ldots \ldots \ldots \ldots$

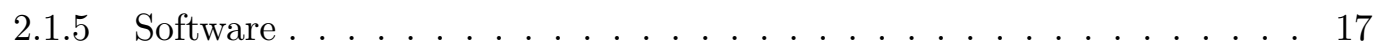

2.2 NMR spectroscopy $\ldots \ldots \ldots \ldots$. . . . . . . . . . . . . . . . . . . 17

$2.2 .1 \quad$ NMR experiments $\ldots \ldots \ldots \ldots \ldots$

$2.2 .2 \quad$ Chemical shift assignment $\ldots \ldots \ldots \ldots \ldots$

$2.2 .3 \quad$ Backbone chemical shift assignment . . . . . . . . . . . . . . 18

2.2 .4 Secondary structure determination . . . . . . . . . . . . . 21

$2.2 .5 \quad$ Residual dipolar couplings (RDCs) . . . . . . . . . . . . . . 21

2.2 .6 NMR relaxation data $\ldots \ldots \ldots \ldots \ldots$

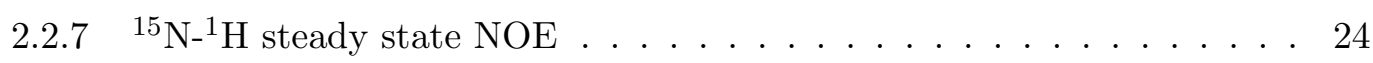

2.2 .8 Measurement of rotational correlation time $\left(\tau_{c}\right) \ldots \ldots$. . . . . 24

$2.2 .9 \quad$ Chemical shift mapping of binding surfaces $\ldots \ldots \ldots$. . . . . . 27

\section{$3 \quad$ Simultaneous measurement of protein one-bond residual} dipolar couplings without increased resonance overlap . 29

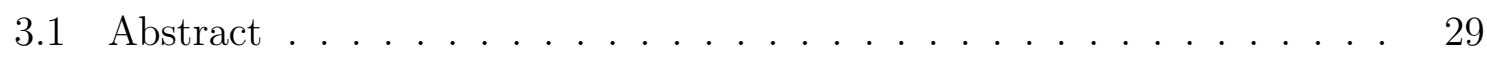

3.2 Introduction . . . . . . . . . . . . . . . . . . . . . . . . 30

$3.3 \quad$ Description of the pulse sequences $\ldots \ldots \ldots$. . . . . . . . . . . . . . . . 31

$3.3 .1 \quad$ TROSY-HNCO experiment . . . . . . . . . . . . . . . . . . . 32

$3.3 .2 \mathrm{CBCA}(\mathrm{CO}) \mathrm{NH}$ experiment $\ldots \ldots \ldots \ldots \ldots$

3.4 Data collection . . . . . . . . . . . . . . . . . . . . . 36

3.5 Results and discussion $\ldots \ldots \ldots$. . . . . . . . . . . . . . . 37

3.5.1 Sensitivity consideration for CBCA(CO)NH experiment . . . . . . . . 37

$3.5 .2 \quad$ Sensitivity consideration for TROSY-HNCO experiment . . . . . . . . 39

3.5 .3 Error estimation . . . . . . . . . . . . . . . . . . . 41

3.5.4 Correlation of measured RDCs with structure . . . . . . . . . . . . . . 43

3.6 Conclusions $\ldots \ldots \ldots$. . . . . . . . . . . . . . . . . . . . . . . . . . . . . . . 43

4 NMR structural studies on the periplasmic domain of DcuS and CitA . . . . . . . . . . . . 45

4.1 Introduction . . . . . . . . . . . . . . . . . . . . . . . . . . . 45 
$4.1 .1 \quad$ Histidine Kinase $(\mathrm{HK})$

4.2 Periplasmic domain of the sensory domain of the two component fumarate sensor DcuS . . . . . . . . . . . . . . . . . . . . . . . . . . 51

4.2 .1 Material and Methods . . . . . . . . . . . . . . . . . . . 52

4.2 .2 Results and discussions $\ldots \ldots \ldots \ldots \ldots$. . . . . . . . . . . 54

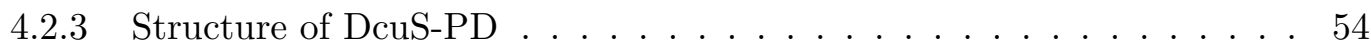

$4.2 .4 \quad$ Binding studies on DcuS-PD $\ldots \ldots \ldots \ldots$. . . . . . . . . . . 57

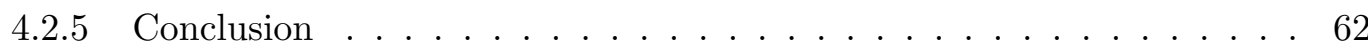

$4.3 \quad$ Periplasmic domain of the sensory domain of the two component citrate sensor CitA . . . . . . . . . . . . . . . . . . . . . 64

4.3 .1 Introduction . . . . . . . . . . . . . . . . . . . . . 64

4.3 .2 Materials and Methods . . . . . . . . . . . . . . . . . 66

4.3 .3 Results and Discussion $\ldots \ldots \ldots$. . . . . . . . . . . . . . 69

4.3 .4 X-ray structure of citrate bound form of CitAP without molybdate . 72

$4.3 .5 \quad$ X-ray structure of citrate free form of CitA $\ldots \ldots \ldots 76$

4.3 .6 NMR studies on the citrate free form of CitAP $\ldots \ldots$. . . . . . . 77

4.3 .7 NMR studies on the citrate bound form of CitAP . . . . . . . . 80

$4.3 .8 \quad$ Sodium binding to CitAP $\ldots \ldots \ldots$. . . . . . . . . . 82

$4.3 .9 \quad$ Residual dipolar coupling analysis $\ldots \ldots$. . . . . . . . . . . . 83

4.3 .10 Comparison of citrate free CitAP and citrate bound CitAP using Xray and NMR . . . . . . . . . . . . . . . . . . . . 86

4.3 .11 Mechanism of Signal Transduction . . . . . . . . . . . . . . . . . . . . 91

4.3 .12 Comparison of structures of DcuS-PD and CitAP . . . . . . . . 93

4.3 .13 Conclusion $\ldots \ldots \ldots \ldots \ldots$

5 Summary and outlook . . . . . . . . . . . . 99

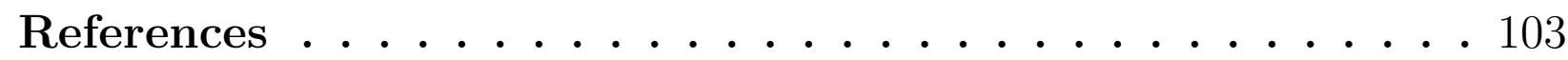

Appendix

A Time evolution of product operators . . . . . . . . 121 
xiv

B Chemical shift assignments and residual dipolar couplings. . . . . . . . . . . . . . . . . 125

B.1 Chemical shift Assignments . . . . . . . . . . . . . . . 125

B.2 Residual dipolar coupling . . . . . . . . . . . . . . . . . . . . . . . . . 130

C Bruker Pulseprogramme and MATHEMATICA scripts 151 C.1 MATHEMATICA scripts . . . . . . . . . . . . . . . . . . . . 159 


\section{List of Figures}

1.1 Examples of coherence transfer pathways . . . . . . . . . . . . . . . 3

1.2 Spin system of a peptide backbone . . . . . . . . . . . . . . . . . . . . . 4

1.3 Pulse sequence for HNCO experiment . . . . . . . . . . . . . . . . 5

1.4 Magnetization transfer pathway for $\mathrm{HNCO}$ experiment . . . . . . . . . . . 6

1.5 Magnetization transfer pathway for $\mathrm{HN}(\mathrm{CA}) \mathrm{CO}$ experiment $\ldots$. . . . . . . 7

1.6 Magnetization transfer pathway for HNCACB experiment . . . . . . . . . 8

$1.7 \quad$ Magnetization transfer pathway for $\mathrm{CBCA}(\mathrm{CO}) \mathrm{NH}$ experiment. . . . . . . . 9

1.8 Coordinate system for internuclear vector orientation with external magnetic field . . . . . . . . . . . . . . . . . . . . . . . 12

$2.1 \quad$ Pulse sequence for measuring cross correlated relaxation rate of ${ }^{15} \mathrm{~N}$. . . . $\quad 25$

3.1 Pulse scheme of the modified 3D TROSY-HNCO experiment for simultaneous measurement of ${ }^{1} \mathrm{~J}_{C^{\prime} N}$ and ${ }^{1} J_{N H}$ couplings . . . . . . . . . . . . . . . 34

3.2 Pulse scheme of the 3D CBCA(CO)NH quantitative $J_{C H}$ and $J_{C \alpha C^{\prime}}$ experiment 35

3.3 Selected region of TROSY-HNCO spectrum . . . . . . . . . . . . . . . 37

3.4 Selected region of $\mathrm{CBCA}(\mathrm{CO}) \mathrm{NH}$ spectrum $\ldots \ldots \ldots$. . . . . . . . 38

3.5 Correlation between observed dipolar couplings and values back-calculated from the crystal structure of ubiquitin . . . . . . . . . . . . . . . . 42

3.6 Correlation of dipolar and J couplings measured for analysing systematic errors 42

4.1 Two component system $\ldots \ldots \ldots \ldots$

4.2 Histidine kinase $\ldots \ldots \ldots$. . . . . . . . . . . . . . . . . . . . . . 47

4.3 The kinase core of Histidine Kinases $\ldots \ldots \ldots$. . . . . . . . . . . . . . 47

4.4 Structure of PYP PAS domain . . . . . . . . . . . . . . . . . . . . . 49

4.5 DcuS-DcuR system . . . . . . . . . . . . . . . . . . . . . . . . . . 52

$4.6 \quad$ Secondary structure of DcuS-PD . . . . . . . . . . . . . . . . . . . . 54 
4.7 Solution structure of DcuS-PD $\ldots \ldots \ldots \ldots \ldots \ldots$

4.8 Comparison of the secondary structure of DcuS-PD and PYP . . . . . . . . 56

4.9 Plot of Het-NOE values measured for DcuS-PD . . . . . . . . . . . 57

4.10 Ratio of peak intensities in fumarate titration with DcuS-PD . . . . . . . . . 58

4.11 Chemical shift changes in tartrate titrations with DcuS . . . . . . . . . . 58

4.12 Structure of DcuS-PD with residues most affected by fumarate binding and tartrate binding. . . . . . . . . . . . . . . . . . . . . 59

4.13 Electrostatic surface potential of DcuS-PD . . . . . . . . . . . . . . . . 59

4.14 Comparison of the amino acid sequences of the periplasmic sensor domains of C4-dicarboxylate or citrate sensory histidine kinases . . . . . . . . . . . . . . 61

4.15 HSQCs of DcuS-PD mutants . . . . . . . . . . . . . . . . . . 63

4.16 The cit regulon of Klebsiella pneumoniae . . . . . . . . . . . . . . . . . . 64

4.17 X-ray structure of GJ dimer of CitAP in complex with citrate and molybdate 65

$4.18{ }^{15} \mathrm{~N}^{1} \mathrm{H}$ HSQC spectra of different construct of CitAP used in the study and the ${ }^{15} \mathrm{~N}-{ }^{1} \mathrm{H}$ HSQC spectrum of the CitAP shorter construct at a salt concentration of $300 \mathrm{mM} \ldots \ldots \ldots \ldots \ldots$

4.19 Overlay of ${ }^{15} \mathrm{~N}-^{1} \mathrm{H}$ HSQC spectra of molybdate titration. . . . . . . . . . . . 71

$4.20{ }^{15} \mathrm{~N}-{ }^{1} \mathrm{H}$ HSQC spectrum of citrate free CitAP with assignments . . . . . . . . 71

$4.21{ }^{15} \mathrm{~N}^{-1} \mathrm{H}$ HSQC spectrum of citrate bound CitAP with assignments . . . . . . 72

4.22 CitAP bound structure . . . . . . . . . . . . . . . . . . . . . . . . . . 73

4.23 X-ray structure of citrate bound CitAP . . . . . . . . . . . . . . . 73

4.24 Superposition of GJ type dimer of 1P0Z and the dimer of new citrate bound CitAP structure . . . . . . . . . . . . . . . . . . . . . . . 75

4.25 X-ray structure of Citrate-free CitAP . . . . . . . . . . . . . . . . 76

$4.26{ }^{13} \mathrm{C}-{ }^{1} \mathrm{H}$ HSQC spetrum of the methyl region and the ${ }^{13} C_{\alpha^{-}}{ }^{1} \mathrm{H}_{\alpha}$ projection of HACACO experiment . . . . . . . . . . . . . . . . . . 78

4.27 Plot of measured correlation time of individual residues of citrate free CitAP 79

4.28 Plot of Heteronuclear ${ }^{15} \mathrm{~N}^{1} \mathrm{H}$ NOE values of citrate free CitAP . . . . . . . . 80

4.29 Secondary structure of citrate bound-CitAP $\ldots \ldots \ldots$. . . . . . . . . . 81

4.30 Citrate bound CitAP dimer with the unassigned residues . . . . . . . . . . . 81 
4.31 Effect of $\mathrm{Na}^{+}$on citrate bound-CitAP $\ldots \ldots \ldots \ldots$. . . . . . . 83

4.32 Correlation between measured H-N RDCs of citrate bound CitAP with back calculated RDCs from different crystal structures of CitAP . . . . . . . . . 84

4.33 Correlation of measured RDCs of citrate free-CitAP with back calculated RDCs from different Xray structures of CitAP and their combinations . . . . 87

4.34 Conformational differences between citrate-bound and citrate-free CitAP . . 88

4.35 Difference between citrate free CitAP and citrate bound CitAP . . . . . . 89

4.36 Structural and dynamic changes in CitAP upon binding to citrate as monitored by multidimensional NMR spectroscopy $\ldots \ldots \ldots$. . . . . . . . . . 90

4.37 Signal transduction mechanism in CitA . . . . . . . . . . . . . . . . . 92

4.38 Superposition of DcuS-PD structure with citrate-free and bound structures of

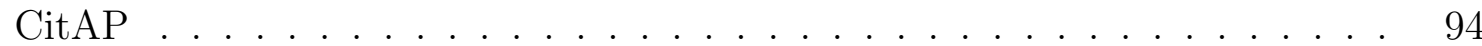

4.39 Conformational exchange line broadening of DcuS-PD and CitAP . . . . . 95 


\section{List of Tables}

2.1 Table of software used in the study . . . . . . . . . . . . . . . . . 17

2.2 Table of experiments measured for the citrate free CitAP sample that was produced in Jülich . . . . . . . . . . . . . . . . . . . . . . . . . . . . 19

2.3 Table of experiments measured for the citrate bound CitAP sample that was produced in Jülich . . . . . . . . . . . . . . . . . . . . . . . . . . . 19

2.4 Table of experiments measured for the citrate free CitAP sample that was produced in our laboratory . . . . . . . . . . . . . . . . . . . . . . 20

2.5 Table of experiments measured for the citrate bound CitAP sample that was produced in our laboratory . . . . . . . . . . . . . . . . . . . . 20

2.6 Compilation of physical constants . . . . . . . . . . . . . . . . 26

A.1 Basis operators for $q=0,1$ or 2 for an $I S$ system of spin $1 / 2 \ldots$. . . . . . . . 122

A.2 Time evolution of common product operators for a two spin systems under different spin hamiltonians . . . . . . . . . . . . . . . . . . . . . . 122

B.1 Backbone chemical shifts of citrate free CitAP at 310K. . . . . . . . . . . . . 125

B.2 Backbone chemical shifts of citrate bound CitAP at 310K. . . . . . . . . . 127

B.3 Residual dipolar couplings measured for DcuS-PD in phages . . . . . . . . . 130

B.4 Residual dipolar couplings measured for Ubiquitine in Otting phase . . . . . 139

B.5 Residual dipolar couplings measured for citrate bound form of CitAP in phages 145

B.6 Residual dipolar couplings measured for citrate free form of CitAP in phages 147 


\section{List of Abbreviations}

ADP

ADP

$\mathrm{CBCA}(\mathrm{CO}) \mathrm{NH}$

CCR

COS-CT

CPMG

CSA

FID

HACACO

HCCH-TOCSY

HK

HNCA

HNCACB

$\mathrm{HN}(\mathrm{CA}) \mathrm{CO}$

HNCO

HSQC

INEPT

IPAP

NMR

NOE

NOESY
Adenosine di-phosphate

Adenosine tri-phosphate

NMR experiment observing peptide ${ }^{15} \mathrm{~N},{ }^{1} \mathrm{H}^{N},{ }^{13} C_{\alpha}$ and ${ }^{13} C_{\beta}$

Cross correlated relaxation

Coherence order transfer

Carr-Purcell-Meiboom and Gill experiment for relaxation studies

Chemical shift anisotropy

Free induction decay

NMR experiment observing correlations between peptide ${ }^{1} \mathrm{H}_{\alpha}$, ${ }^{13} C_{\alpha}$ and $\mathrm{CO}$

TOCSY experiment using $\mathrm{H}-\mathrm{C}-\mathrm{C}-\mathrm{H}$ magnetization transfer pathway

Histidine Kinase

NMR experiment observing peptide ${ }^{15} \mathrm{~N},{ }^{1} \mathrm{H}^{N}$ and ${ }^{13} C_{\alpha}$

NMR experiment observing peptide ${ }^{15} \mathrm{~N},{ }^{1} \mathrm{H}^{N}{ }^{13} C_{\alpha}$ and ${ }^{13} C_{\beta}$

NMR experiment observing peptide ${ }^{15} \mathrm{~N},{ }^{1} \mathrm{H}^{N}$ and ${ }^{13} \mathrm{C}^{\prime}$

NMR experiment observing peptide ${ }^{15} \mathrm{~N},{ }^{1} \mathrm{H}^{N}$ and previous $\mathrm{CO}$

Heteronuclear single-quantum correlation

Insensitive nuclei enhanced by polarization transfer

Inphase-Antiphase experiment to measure $\mathrm{J}$ couplings

Nuclear Magnetic Resonance

Nuclear Overhauser Effect

NOE Spectroscopy 
PAS

PD

$\mathrm{pdb}$

PYP

RDC

rf

ROESY

SVD

TROSY
Principal axis system and also Per-Arnt Sim domain

Periplasmic domain

Protein data bank

Photoactive yellow protein

Residual dipolar coupling

Radio frequency

Rotational frame NOE Spectroscopy

Singular Value Decomposition

Transverse relaxation optimized spectroscopy 


\section{Chapter 1}

\section{Introduction}

\subsection{General Introduction}

Nuclear Magnetic Resonance (NMR) that originated approximately 60 years ago primarily as a potentially accurate method for measuring nuclear magnetogyric ratios (Felix Bloch and E.M Purcell, Nobel prize in physics, 1952) [1, 2]. But it turned out to be a drawback, when it transpired that the rf magnetic susceptibility measured could be a quite a complicated function, exhibiting many sharp, close lying resonances.

However, when it was realized that this complexity rather subtly reflected exceedingly fine characteristics of the electronic environment in which the nuclei are embedded [3], NMR began being developed as a high resolution spectroscopic technique for the elucidation of molecular structure, dynamics and spatial distribution (i.e NMR imaging).

Development of NMR for biological application, mainly protein structural studies were hampered by the poor sensitivity and crowding of signals in the NMR spectra due to the multitude of resonances arising from the protein. The first, three dimensional structure of a small protein was solved in the lab of Kurt Wuthrich ( Nobel prize in chemistry, 2002) [4] in 1985. This was made possible because of the invention of two dimensional fourier transform spectroscopy ( Richard R Ernst, Noble prize in chemistry 1991) [5, 6]. Since then in last two decades major improvements in NMR hardware (magnetic field strength, cryo-probes) and NMR methodology, combined with the availability of molecular biology and biochemical methods for the preparation, and isotope labeling of recombinant proteins have dramatically increased the application of NMR spectroscopy for the characterization of structure and 
dynamics of biological molecules in solution. These improvements were designed to overcome the main problems with NMR of biomolecules, namely the signal to noise ratio and spectral overlap. Isotope labeling (incorporating magnetically active spin like ${ }^{13} \mathrm{C}$ and ${ }^{15} \mathrm{~N}$ into biomolecules) [7, 8, 9, 10, 11, 12, 13, 14, 15, 16], and multi dimensional NMR experiments [17, 18, 19] have become a common procedure for protein NMR analysis. A suite of multidimensional NMR experiments have become routine experiments for the spectroscopic characterization of molecules [20]. Experiments have been developed for the determination of distance restraints derived from quantification of NOESY, and ROESY spectra as well as torsional angle restraints from measurement of scalar ${ }^{3} J$ coupling constants [21]. These traditional NMR parameters provide, short to medium range structural restraints for structural calculation of proteins. During the last decade these traditional NMR parameters were augmented by measurement and interpretation of anisotropic parameters such as residual dipolar couplings (RDCs) and chemical shift anisotropy for structural studies and structural refinement [22].

In this chapter an introduction to three dimensional experiments measured for protein back bone assignment is given. A brief description of RDCs is also presented with emphasis on the determination of the alignment tensor for proteins with already a known structure.

\subsection{Backbone chemical shift assignment}

The first step in NMR investigation of biomolecules is to assign the frequencies of all atoms in a molecule. Backbone chemical shift assignments of protein serves as a starting point for studies of its structure, dynamics and binding properties. Powerful multidimensional experiments have been developed by which the resonance position of all the NMR active, spin half atoms, namely ${ }^{1} \mathrm{H},{ }^{15} \mathrm{~N}$ and ${ }^{13} \mathrm{C}$ for a protein can be assigned. The underlying principles for these experiments are correlation experiments that employ the transfer of coherences.

Coherence transfer is realized using modules in pulse sequence. The most common coherence transfer module used in multidimensional NMR experiment for backbone assignment is 


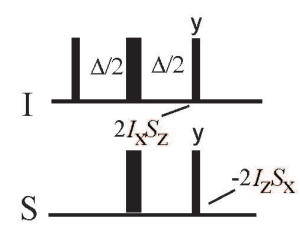

INEPT

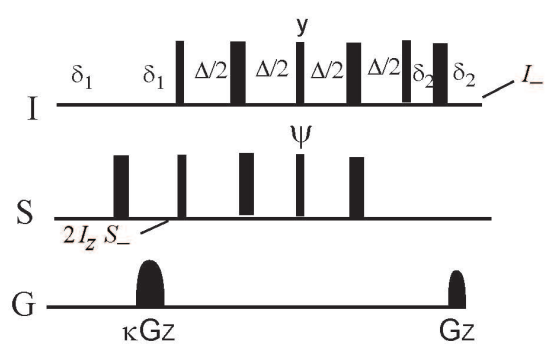

COS-CT

Figure 1.1: INEPT(left) and COS-CT(right) pulse sequence modules that realize coherence transfers, that are incorporated into many multidimensional NMR experiments. In the COS-CT module, concomitant with the change of the pulse with phase $\psi(\mathrm{y}$ to $-\mathrm{y})$, sign of either one of the gradient is changed to give two specific coherence transfer scheme (see Equation 1.2 and 1.3). The value of $\kappa$ depends on the ratio of gyromagnetic ratios of I and S spins. $\Delta=1 / 2 \mathrm{~J}_{I S}$

the INEPT (Insensitive Nuclei Enhanced by Polarization Transfer) [23] coherence transfer scheme (refer Figure 1.1). This can be written in product operator term [24] as

$$
2 I_{x} S_{z} \stackrel{90_{y}^{(I, S)}}{\longrightarrow}-2 I_{z} S_{x}
$$

where $I$ and $S$ are the heteronuclear (having different frequencies; different gyromagnetic ratios) spin half particles. $90_{y}^{(I, S)}$ is the radio frequency pulse(rf) applied along the y axis on both the spins simultaneously with a power corresponding to the rotation of initial magnetization by $90^{\circ}$.

Another type of coherence transfer used extensively for signal enhancement in multidimensional NMR, makes use of a module which changes the coherence order (denoted by $p$ ) of the initial and final operator in a specific (designed) way.

$$
\begin{aligned}
& \text { Antiecho: } I_{z} S_{-} e^{i \Omega_{S} t_{1}} \stackrel{C O S, t_{2}}{\longrightarrow} I_{-} e^{i \Omega_{S} t_{1}} e^{i \Omega_{I} t_{2}} \\
& \text { Echo: } I_{z} S_{+} e^{-i \Omega_{S} t_{1}} \stackrel{\text { COS, t }}{\longrightarrow} I_{-} e^{-i \Omega_{S} t_{1}} e^{i \Omega_{I} t_{2}}
\end{aligned}
$$

where $S_{ \pm}=S_{x} \pm i S_{y}$ and $I_{ \pm}=I_{x} \pm i I_{y}$ and $\Omega_{I, S}$ is the chemical shift (frequency) of corresponding nucleus its associated with. In equation 1.2 coherence order transfer is from 


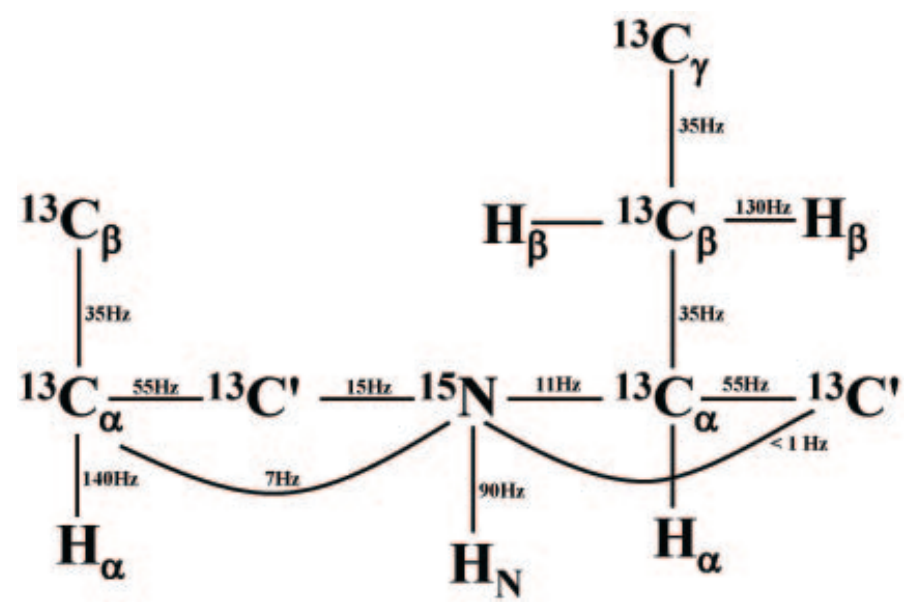

Figure 1.2: Spin system of a peptide backbone and the size of ${ }^{1} J$ and ${ }^{2} J$ coupling constants that are used for magnetization transfer in ${ }^{13} \mathrm{C}-,{ }^{15} \mathrm{~N}$-labeled proteins

$p=-1$ to $p=-1$ and for equation 1.3 from $p=+1$ to $p=-1$. This type of transfer is commonly used in three dimensional experiments to increase signal sensitivity. Such modules are called PEP ( Preservation of Equivalent Pathways) or COS (Coherence Order Selective transfer) [25, 26, 27, 28]. Usually these modules are combined with gradient selection for maximum sensitivity and water suppression (Figure 1.1). For multi-dimensional experiments with only one COS transfer, a gain of $\sqrt{2}$ in $\mathrm{S} / \mathrm{N}$ is obtained with gradient selection [26].

Assignment strategies for non isotopically labeled proteins realizes on the transfer of magnetization in the homonuclear spins (spins of same $\gamma^{\prime}$ s, here ${ }^{1} \mathrm{H}$ ) [29]. The sequential connectivity relies on the occurrence of resonance frequency of $\mathrm{H}_{\alpha}(i)$ proton of amino acid $(i)$ in the ${ }^{1} \mathrm{H}^{N}(i), \mathrm{H}_{\alpha}(i)$ cross peak in the COSY spectrum and in ${ }^{1} \mathrm{H}^{N}(i+1), \mathrm{H}_{\alpha}(i)$ cross peak in NOESY spectrum. Such an assignment strategy is limited to small proteins because of the poor resolution of $\mathrm{H}_{\alpha}$ spins (2 ppm), and the conformational dependence of the NOESY spectra. These difficulties are overcome in the assignment strategies for ${ }^{13} \mathrm{C}-,{ }^{15} \mathrm{~N}$-labeled proteins, since coherence is transfered via the one bond coupling $\left({ }^{1} J\right)$ which is considerably larger and less prone to conformational dependency than the ${ }^{3} J$ coupling used in the 
homonuclear approach. Signals can also be dispersed in multi dimensions, hence providing lesser crowding in the spectra. Larger ${ }^{1} J$ coupling also shortens the coherence transfer delays, making it favorable for the measurement of larger system in which the magnetization relaxes relatively faster. Figure 1.2 shows the spin system of peptide backbone and indicates the size of coupling constants used for magnetization transfer in a doubly ${ }^{13} \mathrm{C}-,{ }^{15} \mathrm{~N}-$ labeled proteins. For small to medium size proteins $(4 \mathrm{kDa}-20 \mathrm{kDa})$, a set of four experiments are to be measured for the backbone assignments. These are briefly described in the following subsections.

\subsubsection{HNCO}

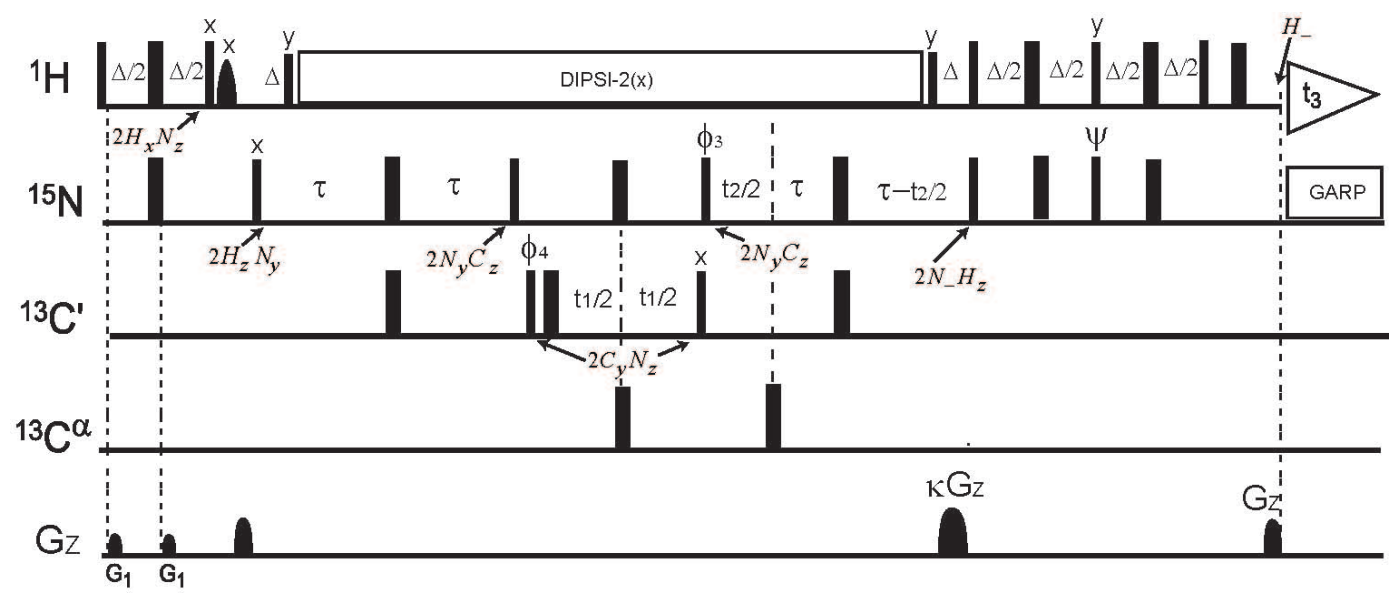

Figure 1.3: Pulse scheme for a sensitivity enhanced three dimensional HNCO experiment with relevant product operators given in different time point of the sequence. $180^{\circ}$ and $90^{\circ}$ pulses are represented by broad and narrow bars. $\Delta=1 / 2^{1} \mathrm{~J}_{N H}$ and $2 \tau=1 / 2{ }^{1} \mathrm{~J}_{C^{\prime} N}$. The phases of all pulses are $x$ unless specified. $\phi 3=2(x), 2(-x), \phi 4=x,-x, \psi=\mathrm{y}$ and receiver $=x, 2(-x), x$. Quadrature detection in ${ }^{13} \mathrm{C}$ ' dimension is obtained by STATES-TPPI method by incrementing the phase of $\phi 4$. Echo-anti echo method is used in the ${ }^{15} \mathrm{~N}$ dimension by shifting the phase of $\psi$ from $y$ to $-y$ with the inversion of the sign of one of the gradient $G_{z} \cdot G_{z}$ is the gradient strength in $\mathrm{G} / \mathrm{cm}$ with $\kappa=\gamma_{H} / \gamma_{N}= \pm 10$

The HNCO experiment is the prototype of all triple resonance experiments. It correlates the ${ }^{13} \mathrm{C}^{\prime}$ resonances of an amino acid residue with the ${ }^{1} \mathrm{H}^{N}$ and ${ }^{15} \mathrm{~N}$ resonances of the following residue. Schematic representation of the pulse sequence for the HNCO experiment is given in the Figure 1.3. Magnetization transfer pathway is shown in Figure 1.4. Starting at an 


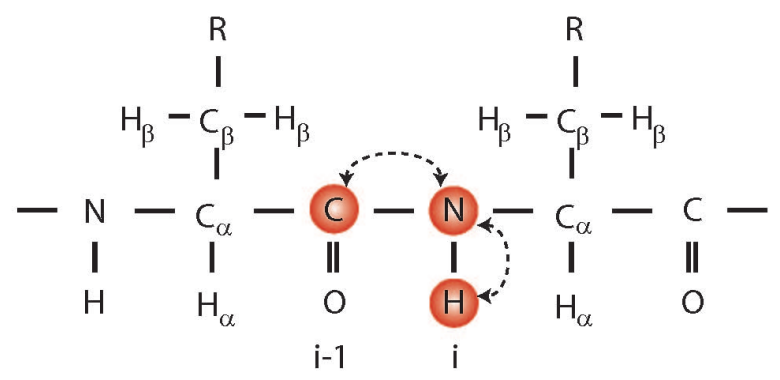

Figure 1.4: HNCO experiment: The magnetization is transferred from the ${ }^{1} \mathrm{H}^{N}(i) \rightarrow{ }^{15} \mathrm{~N}(i) \rightarrow$ ${ }^{13} \mathrm{C}^{\prime}(i-1)$ and then comes back to ${ }^{1} \mathrm{H}^{N}(i)$ along the same path. The frequencies of ${ }^{1} \mathrm{H}^{N}(i),{ }^{15} \mathrm{~N}(i)$ and ${ }^{13} \mathrm{C}^{\prime}(i-1)$ (red) are observed.

${ }^{1} \mathrm{H}^{N}$, the magnetization is transferred to the directly attached ${ }^{15} \mathrm{~N}$ through an INEPT step. The initial operator for the INEPT transfer is obtained by the evolution of the proton magnetization under the heteronuclear weak scalar coupling hamiltonian $\left(2^{1} J_{N H} H_{z} N_{z}\right.$ ) (refer appendix A). After the INEPT transfer, ${ }^{15} \mathrm{~N}$ magnetization evolves under the ${ }^{1} \mathrm{~J}_{C^{\prime} N}$ coupling to the $N_{x} C_{z}^{\prime}$ term, which is converted to $N_{z} C_{y}^{\prime}$ with the next INEPT transfer. The C' magnetization $\left(N_{z} C_{y}^{\prime}\right)$ is frequency labeled and transferred back to ${ }^{15} \mathrm{~N}$ using a reverse INEPT step. During the subsequent constant time delay the ${ }^{1} \mathrm{~J}_{C^{\prime} N}$ coupling is refocused together with the frequency labeling of ${ }^{15} \mathrm{~N}$. During the following delays and pulses the ${ }^{15} \mathrm{~N}$ anti-phase magnetization $\left(H_{z} N_{-}\right)$is transferred to its directly attached ${ }^{1} \mathrm{H}^{N}$ single quantum coherence $\left(H_{-}\right)$using the COS or PEP transfer. This is an out and back experiment [30] with the magnetization starting on ${ }^{1} \mathrm{H}^{N}$ and being detected also in the same ${ }^{1} \mathrm{H}^{N}$. This scheme uses echo-antiecho [31] with sensitivity enhancement for quadrature detection in the ${ }^{15} \mathrm{~N}$ dimension.

\subsection{2 $\mathrm{HN}(\mathrm{CA}) \mathrm{CO}$}

The $\mathrm{HN}(\mathrm{CA}) \mathrm{CO}$ experiment provides sequential correlations between the ${ }^{1} \mathrm{H}^{N}$ and ${ }^{15} \mathrm{~N}$ chemical shifts of one amino acid residue and the ${ }^{13} \mathrm{C}$ ' chemical shift of the same as well as preceding residue by transferring coherence via the intervening ${ }^{13} C_{\alpha}$ spin (Figure 1.5). In this 
experiment, $C^{\prime}(i), C^{\prime}(i-1), N(i)$, and $H^{N}(i)$ resonances are observed. Just like the HNCO experiment, the ${ }^{1} \mathrm{H}^{N}$ magnetization is transferred to its directly attached ${ }^{15} \mathrm{~N}$ using an INEPT step. The ${ }^{15} \mathrm{~N}$ magnetization evolves with ${ }^{1} J_{C_{\alpha} N}$ and ${ }^{2} J_{C_{\alpha} N}$ coupling constants to its directly attached ${ }^{13} C_{\alpha}$ and ${ }^{13} C_{\alpha}(i-1)$ of preceding amino acids respectively, and from there to their directly attached ${ }^{13} \mathrm{C}^{\prime}$ spins. After frequency labeling the ${ }^{13} \mathrm{C}^{\prime}$ resonances, the magnetization is transferred back to ${ }^{13} C_{\alpha}$ resonances and then back to ${ }^{15} \mathrm{~N}$ using reverse INEPT transfer steps using the ${ }^{1} J_{C_{\alpha} N}$ and ${ }^{2} J_{C_{\alpha} N}$ couplings. Like in HNCO experiment the ${ }^{15} \mathrm{~N}$ anti-phase magnetization is transferred to its directly attached ${ }^{1} \mathrm{H}^{N}$ spins using the COS-CT transfer step.

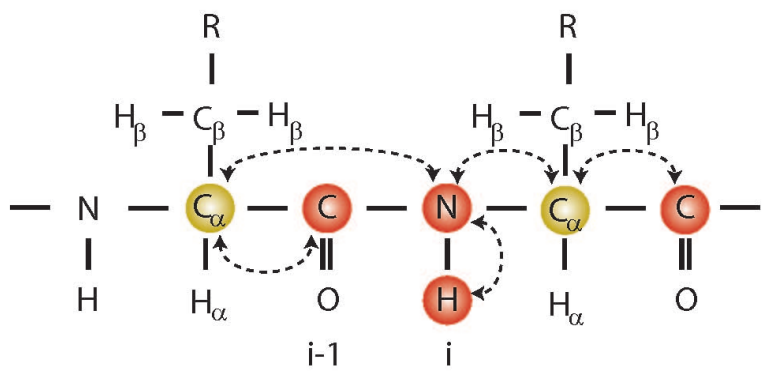

Figure 1.5: $\mathrm{HN}(\mathrm{CA}) \mathrm{CO}$ experiment: The magnetization is transferred from the ${ }^{1} \mathrm{H}^{N}(i) \rightarrow{ }^{15} \mathrm{~N}(i)$ $\rightarrow{ }^{13} C_{\alpha}(i) \rightarrow{ }^{13} \mathrm{C}^{\prime}(i-1) /{ }^{13} \mathrm{C}^{\prime}(i)$ and then comes back to ${ }^{1} \mathrm{H}^{N}(i)$ along the same pathway. The ${ }^{13} C_{\alpha}$ (yellow) acts only as relay nucleus, its frequency is not detected. The frequencies of ${ }^{1} \mathrm{H}^{N},{ }^{15} \mathrm{~N}$ and ${ }^{13} \mathrm{C}^{\prime}$ (red) are observed.

\subsubsection{HNCACB}

The HNCACB experiment correlates the ${ }^{13} C_{\alpha}$ and ${ }^{13} C_{\beta}$ resonances with the ${ }^{1} \mathrm{H}^{N}$ and ${ }^{15} \mathrm{~N}$ resonances of the same residue and the ${ }^{1} \mathrm{H}^{N}$ and ${ }^{15} \mathrm{~N}$ resonances of the neighboring residue (Figure 1.6 ). The magnetization transfer is similar to the HNCACO experiment till the ${ }^{13} C_{\alpha}$ resonance, after which the $C_{\alpha}(i)$ and $C_{\alpha}(i-1)$ resonances are partially transferred to their attached ${ }^{13} C_{\beta}$ resonances via the ${ }^{1} \mathrm{~J}_{C_{\alpha} C_{\beta}}$ coupling. ${ }^{13} C_{\alpha}$ and ${ }^{13} C_{\beta}$ resonances are frequency labeled and then transferred to the attached ${ }^{15} \mathrm{~N}$ spins and from there to ${ }^{1} \mathrm{H}^{N}$ spins using similar magnetization scheme to HNCO. In this experiment, $\mathrm{C}^{\beta}(i), \mathrm{C}^{\beta}(i-1)$, $\mathrm{C}^{\alpha}(i), \mathrm{C}^{\alpha}(i-1), \mathrm{N}(i)$, and $\mathrm{H}^{N}(i)$ resonances are observed. For a medium-sized protein $(\sim$ 
$15 \mathrm{kDa}$ ), this experiment alone can provide virtually complete sequential assignment of the ${ }^{1} \mathrm{H}^{N},{ }^{15} \mathrm{~N},{ }^{13} C_{\alpha}$, and ${ }^{13} C_{\beta}$ resonances, because in addition to the sequential connectivities, the ${ }^{13} C_{\alpha}$ and ${ }^{13} C_{\beta}$ chemical shifts provide information on the amino acid type.

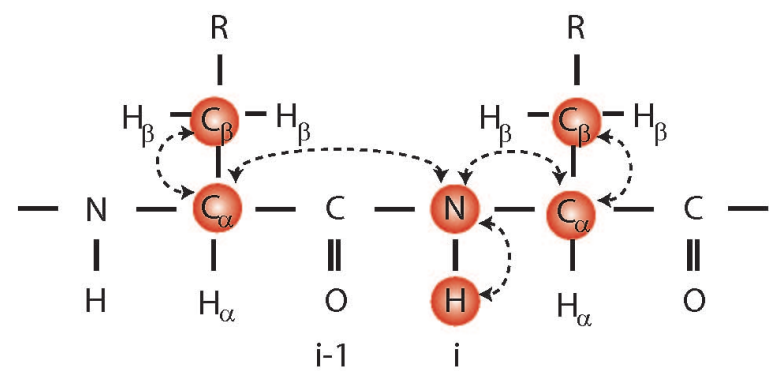

Figure 1.6: HNCACB experiment: The magnetization is transferred from the ${ }^{1} \mathrm{H}^{N}(i) \rightarrow{ }^{15} \mathrm{~N}(i)$ $\rightarrow{ }^{13} C_{\alpha}(i) /{ }^{13} C_{\alpha}(i-1)$ and then from there to ${ }^{13} C_{\beta}(i) /{ }^{13} C_{\beta}(i-1)$. After which it comes back to ${ }^{1} \mathrm{H}^{N}(i)$ along the same path. The frequencies of ${ }^{1} \mathrm{H}^{N}(i),{ }^{15} \mathrm{~N}(i),{ }^{13} C_{\alpha}(i),{ }^{13} C_{\beta}(i),{ }^{13} C_{\alpha}(i-1)$ and ${ }^{13} C_{\beta}(i-1)$ (red) are observed.

\subsection{4 $\mathrm{CBCA}(\mathrm{CO}) \mathrm{NH}$}

The $\mathrm{CBCA}(\mathrm{CO}) \mathrm{NH}$ experiment correlates both the ${ }^{13} C_{\alpha}$ and ${ }^{13} C_{\beta}$ resonances of an amino acid residue with the ${ }^{1} \mathrm{H}^{N}$ and ${ }^{15} \mathrm{~N}$ resonances of the proceeding residue (Figure 1.7). In this experiment instead of starting on the ${ }^{1} \mathrm{H}^{N}$, magnetization transfer starts with ${ }^{1} \mathrm{H}_{\alpha}$ and ${ }^{1} \mathrm{H}_{\beta}$ spins. Magnetization is transferred to its directly attached ${ }^{13} C_{\alpha}$ and ${ }^{13} C_{\beta}$ with an initial INEPT step followed by chemical shift evolution and then using another INEPT transfer to directly attached ${ }^{13} \mathrm{C}^{\prime}$ spins. The ${ }^{13} \mathrm{C}^{\prime}$ spins evolves with the ${ }^{1} \mathrm{~J}_{N C^{\prime}}$ to its directly attached ${ }^{15} \mathrm{~N}$ which is then frequency labeled and then transferred to the ${ }^{1} \mathrm{H}^{N}$ magnetization using the COS-CT step.

\subsubsection{Assignment strategy}

From the combination of $\mathrm{CBCA}(\mathrm{CO}) \mathrm{NH}$ and $\mathrm{HNCACB}$ experiments backbone resonance assignments and the sequential connectivities can be obtained. These experiments are sensitive enough for medium size proteins ( $\sim 15 \mathrm{kDa}, 130$ amino acids) and provide the ${ }^{13} C_{\alpha}$ and 


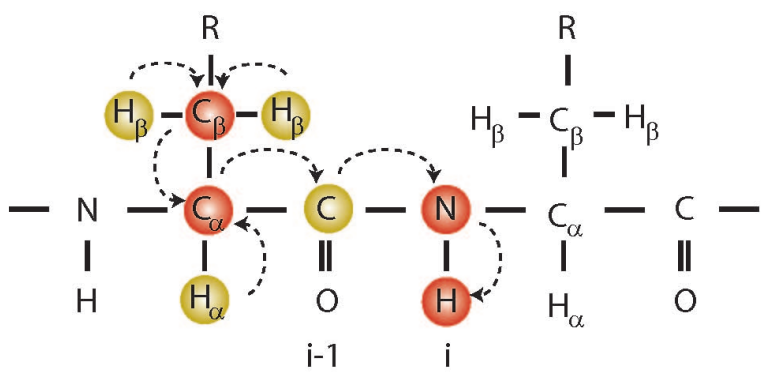

Figure 1.7: $\mathrm{CBCA}(\mathrm{CO}) \mathrm{NH}$ experiment: The magnetization is transferred from the ${ }^{1} \mathrm{H}_{\alpha}(i-$ 1) $/{ }^{1} \mathrm{H}_{\beta}(i-1) \rightarrow{ }^{13} C_{\alpha}(i-1) /{ }^{13} C_{\beta}(i-1) \rightarrow{ }^{13} \mathrm{C}^{\prime}(i-1) \rightarrow{ }^{15} \mathrm{~N}(i) \rightarrow{ }^{1} \mathrm{H}^{N}(i)$. The ${ }^{1} \mathrm{H}_{\alpha},{ }^{1} \mathrm{H}_{\beta}$ and ${ }^{13} \mathrm{C}^{\prime}$ (yellow) act only as relay nucleus, their frequency are not detected. The frequencies of ${ }^{1} \mathrm{H}^{N}(i)$, ${ }^{15} \mathrm{~N}(i),{ }^{13} C_{\alpha}(i-1)$ and ${ }^{13} C_{\beta}(i-1)$ (red) are observed.

${ }^{13} C_{\beta}$ chemical shifts to establish the sequential link between neighboring residues. Furthermore, when both the ${ }^{13} C_{\alpha}$ and ${ }^{13} C_{\beta}$ chemical shifts are provided at the same time, it gives important information about the amino acid type and secondary structure (e.g. $\alpha$-helix and $\beta$-strand). ${ }^{13} \mathrm{C}$ ' resonances from $\mathrm{HNCO}$ and $\mathrm{HN}(\mathrm{CA}) \mathrm{CO}$ experiments are used to resolve the assignment ambiguities for residues for which ${ }^{13} C_{\alpha}{ }^{13} C_{\beta}$ degeneracies exist.

This type of assignment strategy is used extensively in the proteins studied in this thesis.

\subsection{Residual Dipolar Couplings (RDC)}

One of the major break through in the last decade has been the use of anisotropic interactions like RDC's, CSAs and pseudo contact shifts in solution state NMR for structural studies of biological molecules [22, 32, 33, 34]. This was made possible with the discovery of tunable alignment of macromolecules as solutes in a dilute aqueous liquid crystal [35, 36, 37, 38, 39, 40]. In these media the anisotropic interaction do not average to zero and the residual effect could be measured easily through NMR.

Anisotropic interactions are magnetic properties that depend on the orientation of the molecule with respect to external magnetic field. Hence valuable information can be obtained 
about the shape and orientation of the molecule if one could exploit these anisotropic interaction. RDCs are one such parameter which is comparatively very easy to measure once the molecules are partially aligned with the external magnetic field. A brief description on RDC is given below. Equations can be formulated for the calculation of alignment tensor from a known molecule structure and measured residual dipolar couplings. Equations can also be obtained for predicting dipolar coupling if the three principle component of the alignment tensor are known. The order matrix approach described below is commonly used in the structural validation using RDCs. A good correlation of RDCs measured for a protein in solution and those calculated from a known structure (X-ray or NMR) would indicate a similar structure for molecule in solution also.

Hamiltonian for dipolar coupling between two spins $\left(I_{1}, I_{2}\right)$ is given by

$$
H_{I_{1} I_{2}}^{D D}=D_{\max }\left\{3 \frac{1}{\mathbf{r}_{I_{1} I_{2}}}\left(\mathbf{I}_{1} \cdot \mathbf{r}_{I_{1} I_{2}}\right)\left(\mathbf{I}_{2} \cdot \mathbf{r}_{I_{1} I_{2}}\right)-\mathbf{I}_{1} \cdot \mathbf{I}_{2}\right\}
$$

where $D_{\max }=-\mu_{0} \frac{\gamma_{I} \gamma_{S} \hbar}{4 \pi r_{I_{1} I_{2}}^{3}}$ (Refer Table 2.6 in materials and methods chapter 2 for the values of all the physical constants). Expressing $\mathbf{r}$ in equation 1.4 in spherical coordinates and taking equation 1.4 to a rotating frame, and using the secular approximation, the dipolar hamiltonian for two homonuclear spins will be

$$
H_{I_{1} I_{2}}^{\mathrm{hom}}=D_{\max }\left\{\left[2 I_{1 z} I_{2 z}-\frac{1}{2}\left(I_{1}^{+} I_{2}^{-}+I_{1}^{-} I_{2}^{+}\right)\right] \frac{\left(3 \cos ^{2} \theta-1\right)}{2}\right\}
$$

where $\theta$ is the angle between the magnetic field $B_{0}$ and the internuclear vector connecting spins $I_{1}$ and $I_{2}$. For a weak coupling limit, only the first term in equation 1.4 need to be considered. For two heteronuclear $\operatorname{spins}\left(\mathrm{I}_{1}=\mathrm{I}, \mathrm{I}_{2}=\mathrm{S}\right)$ the dipolar hamiltonian in the rotating frame would be

$$
H_{I S}^{\text {het }}=D_{\max }\left\{\left(2 I_{z} S_{z}\right) P_{2}(\cos \theta)\right\}
$$

where $\frac{\left(3 \cos ^{2} \theta-1\right)}{2}=P_{2}(\cos \theta)$ is the second order Legendre Polynomial.

In solution, $P_{2}(\cos \theta)$ is time averaged due to fast tumbling of the molecule $\left(\left\langle P_{2}(\cos \theta)\right\rangle\right.$ angular brackets denote time averaging). In an isotropic solution all possible orientation of the dipolar couplings are possible (probability of all orientations in a sphere are same) and hence the orientational dependence drops to zero $\left(\int_{0}^{2 \pi} d \phi \int_{0}^{\pi} P_{2}(\cos \theta) d(\cos \theta)=0\right)$. In an 
alignment media, the orientational dependence does not average to zero and hence could be measured with NMR.

Including the time average for orientational dependence in equation 1.6, the RDC for two heteronuclear spins is,

$$
D_{I S}^{r d c}=D_{\max }\left[\left\langle P_{2}(\cos \theta)\right\rangle\right]
$$

\subsubsection{Alignment tensor determination for a rigid molecule}

For a rigid molecule for which the atom positions are already known, the alignment tensor for the molecule can be readily derived [41]. The direction of the internuclear vector $\mathbf{r}_{I S}$ can be given with respect to a cartesian molecular coordinate system by the angles $\phi_{x}, \phi_{y}$ and $\phi_{z}$ (Figure 1.8). In the case of rigid object, the projections $c_{x}=\cos \phi_{x}, c_{y}=\cos \phi_{y}$ and $c_{z}=\cos _{z}$ to axes $\mathrm{x}, \mathrm{y}$ and $\mathrm{z}$ do not change with time and are identical for every molecule with identical structure. This coordinate system is fixed in the molecule and this reorients as molecule tumble in solution. Consequently, the axes $\mathrm{x}, \mathrm{y}$ and $\mathrm{z}$ makes instantaneous angles $\beta_{x}(t), \beta_{y}(t)$ and $\beta_{z}(t)$ with the magnetic field. The time dependence is associated with corresponding projections $\left\langle C_{x}\right\rangle=\cos \beta_{x},\left\langle C_{y}\right\rangle=\cos \beta_{y}$ and $\left\langle C_{z}\right\rangle=\cos \beta_{z}$ along $\mathbf{B}$.

Remembering that the scalar product between two unit vectors is identical to the cosine of the angle $\theta$ between the two vectors we $\operatorname{can}$ write $\cos \theta$ in equation 1.7 as:

$$
\langle\cos \theta\rangle=\mathbf{B}^{\mathrm{T}} \cdot \mathbf{r}=\left(\begin{array}{lll}
\left\langle C_{x}\right\rangle & \left\langle C_{y}\right\rangle & \left\langle C_{z}\right\rangle
\end{array}\right)\left(\begin{array}{c}
c_{x} \\
c_{y} \\
c_{z}
\end{array}\right)
$$

Hence the orientational dependence of the dipolar coupling takes the form

$$
\left\langle P_{2}(\cos \theta)\right\rangle=\frac{\left(3\left\langle\cos ^{2} \theta\right\rangle-1\right)}{2}=\frac{1}{2}\left[\begin{array}{l}
3\left(\left\langle C_{x}\right\rangle^{2} c_{x}^{2}+\left\langle C_{y}\right\rangle^{2} c_{y}^{2}+\left\langle C_{z}\right\rangle^{2} c_{z}^{2}+2\left\langle C_{x} C_{y}\right\rangle c_{x} c_{y}\right. \\
\left.+2\left\langle C_{y} C_{z}\right\rangle c_{y} c_{z}+2\left\langle C_{z} C_{x}\right\rangle c_{z} c_{x}\right)-1
\end{array}\right]
$$

Separating the time dependent and time independent variables, a concise notation is reached by defining a $3 \times 3$ matrix known as Saupe order matrix 42 S such as

$$
\left\langle P_{2}(\cos \theta)\right\rangle=\sum_{i j=\{x, y, z\}} \frac{1}{2}\left[3\left\langle C_{i} C_{j}\right\rangle-\delta_{i j}\right] c_{i} c_{j}=\sum_{i j=\{x, y, z\}} S_{i j} c_{i} c_{j}
$$


where $\delta_{i j}$ is the kronecker delta function. Since $\left\langle C_{i} C_{j}\right\rangle=\left\langle C_{j} C_{i}\right\rangle$ and $\left\langle C_{x}\right\rangle^{2}+\left\langle C_{y}\right\rangle^{2}+\left\langle C_{z}\right\rangle^{2}=1$ the order matrix is symmetric and traceless. Consequently, there are only five independent matrix elements which means that $\mathbf{S}$ can be determined when at least five non-redundant RDCs are available. Substituting $P_{2}(\cos \theta)$ of equation 1.9 in equation 1.7 , the measured RDC will be

$$
D_{I S}^{r d c}=D_{\max } \sum_{i j=\{x, y, z\}} S_{i j} c_{i} c_{j}=D_{\max } \sum_{i j=\{x, y, z\}} S_{i j} \cos \phi_{i} \cos \phi_{j}
$$

Order matrix analysis is basically setting up and solving a system of linear equation of the

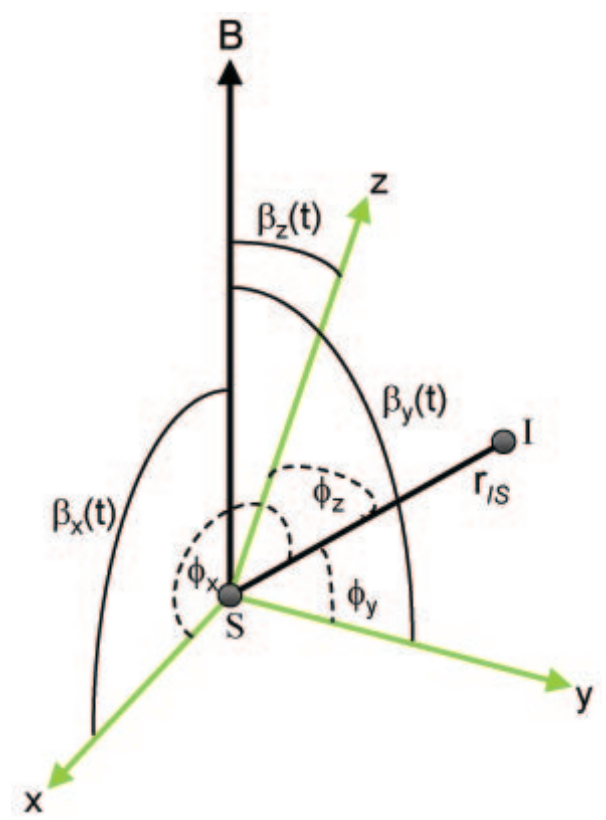

Figure 1.8: Internuclear vector $\mathbf{r}_{I S}$ makes fixed angle $\phi_{x}, \phi_{y}$ and $\phi_{z}$ with the molecular coordinate system (green). As the molecule tumble in solution, the angles of the axes $\mathrm{x}, \mathrm{y}$, and $\mathrm{z}$ denotes by $\beta_{x}, \beta_{y}$ and $\beta_{z}$ with respect to field $\mathbf{B}$ change with time.

type $\mathbf{A X}=\mathbf{b}$ which can be explicitly written in matrix form.

$$
\left(\begin{array}{ccccc}
c_{1, z}^{2}-c_{1, x}^{2} & c_{1, y}^{2}-c_{1, x}^{2} & 2 c_{1, x} c_{1, y} & 2 c_{1, y} c_{1, z} & 2 c_{1, x} c_{1, z} \\
c_{2, z}^{2}-c_{2, x}^{2} & c_{2, y}^{2}-c_{2, x}^{2} & 2 c_{2, x} c_{2, y} & 2 c_{2, y} c_{2, z} & 2 c_{2, x} c_{2, z} \\
\cdot & \cdot & \cdot & \cdot & \cdot \\
\cdot & \cdot & \cdot & \cdot & \cdot \\
\cdot & \cdot & \cdot & \cdot & \cdot \\
c_{n-1, z}^{2}-c_{n-1, x}^{2} & c_{n-1, y}^{2}-c_{n-1, x}^{2} & 2 c_{n-1, x} c_{n-1, y} & 2 c_{n-1, y} c_{n-1, z} & 2 c_{n-1, x} c_{n-1, z} \\
c_{n, z}^{2}-c_{n, x}^{2} & c_{n, y}^{2}-c_{n, x}^{2} & 2 c_{n, x} c_{n, y} & 2 c_{n, y} c_{n, z} & 2 c_{n, x} c_{n, z}
\end{array}\right)\left(\begin{array}{c}
S_{y y} \\
S_{z z} \\
S_{x y} \\
S_{x z} \\
S_{y z}
\end{array}\right)=\left(\begin{array}{c}
D_{\text {red }}^{1} \\
D_{r e d}^{2} \\
\cdot \\
\cdot \\
\cdot \\
D_{\text {red }}^{n-1} \\
D_{\text {red }}^{n}
\end{array}\right)
$$

where $D_{\text {red }}$ is obtained by the division of the measured dipolar coupling with $\mathrm{D}_{\max }$. 
Hence using equation 1.11 elements of the Saupe matrix can be obtained with the knowledge of dipolar coupling and the directional cosines associated with it. Elements of the overdetermined $\mathbf{S}$ are best determined by singular value decomposition (SVD) since the order matrix is symmetric and real [43. Alternatively it can also be obtained by a gradient search or by a fitting program that minimizes the difference between the observed and predicted coupling (details are given in chapter 2).

By diagonalizing the order matrix, it is possible to reduce order parameter description to a principal order parameter, $\mathrm{S}_{z z}$ and an asymmetric parameter $\eta=S_{y y}-S_{x x} / S_{z z}$ where $\left|S_{z z}\right|>\left|S_{y y}\right|>\left|S_{x x}\right|$. The coordinate system in which the Saupe matrix is diagonal is called principal axis system or PAS. In PAS the RDCs are given by

$$
D\left(\phi_{x}, \phi_{y}, \phi_{z}\right)=D_{I S}^{\max } \sum_{i=\{x, y, z\}} S_{i i} c_{i}^{2}
$$

By converting the vector direction $\left(c_{i}\right.$ 's) to polar coordinate; $c_{x}^{2}=\sin ^{2} \vartheta \cos ^{2} \varphi, c_{y}^{2}=$ $\sin ^{2} \vartheta \sin ^{2} \varphi$ and $c_{y}^{2}=\cos ^{2} \vartheta$, RDCs can be written as a function of $\vartheta$ and $\varphi$

$$
D(\vartheta, \varphi)=D_{I S}^{\max }\left[S_{z z} \cos ^{2} \vartheta+S_{x x} \sin ^{2} \vartheta \cos ^{2} \varphi+S_{y y} \sin ^{2} \vartheta \sin ^{2} \varphi\right]
$$

Making use of the definition of $\eta$ and using trigonometric identities like $2 \sin ^{2} \varphi=1-\cos 2 \varphi$ and $2 \cos ^{2} \varphi=1+\cos 2 \varphi$ equation 1.12 can be recast into

$$
D(\vartheta, \varphi)=D_{I S}^{\max } S_{z z}\left[\frac{1}{2}\left(3 \cos ^{2} \vartheta-1\right)+\frac{1}{2} \eta \sin ^{2} \vartheta \cos 2 \varphi\right]
$$

Equation 1.13 can be expressed in terms of alignment matrix $\mathbf{A}$ which is simply Saupe matrix S scaled by $2 / 3$. Writing $A_{a}=S_{z z}$ as the axial component and $\eta=S_{x x}-S_{y y} / S_{z z}=3 / 2 R$ equation 1.13 can be written as

$$
D(\vartheta, \varphi)=D_{I S}^{\max } \frac{A_{a}}{2}\left[\left(3 \cos ^{2} \vartheta-1\right)+\frac{3}{2} R \sin ^{2} \vartheta \cos 2 \varphi\right]
$$

where $\mathrm{R}$ is the measure of non axiality or rhombicity of the alignment tensor. Many proteins are asymmetric in their shape and, thus, their alignment is more or less rhombic, where as stretch of double stranded DNA is nearly axial. 
From equation 1.14 it is clear that one cannot obtain specific directions of an internuclear vector but only possible combinations of $\vartheta$ and $\varphi$ values along the surface of a cone is obtained. Furthermore since the form of D is an even function of $\vartheta$ and $\varphi$ it is impossible to distinguish the symmetry alternative $( \pm \vartheta, \pm \varphi)$. Multivalued $\mathrm{D}(\vartheta, \varphi)$ can be overcome by measuring RDCs in two different alignment media. Writing $D_{I S}^{\max } \frac{A_{a}}{2}=D_{a}$ as the magnitude of the residual dipolar coupling, the measured RDCs can be expressed in the most popular form.

$$
D(\vartheta, \varphi)=D_{a}\left[\left(3 \cos ^{2} \vartheta-1\right)+\frac{3}{2} R \sin ^{2} \vartheta \cos 2 \varphi\right]
$$

\subsubsection{Measurement of the RDCs}

RDCs are measured in the same way as scalar coupling of comparable strength. Because of their larger size, heteronuclear one bond dipolar couplings are usually measured in biomolecules. For heteronculear spins the hamiltonian for evolution of dipolar couplings (see equation 1.6 is very similar to hamiltonian of weak coupling $2 \pi J I_{z} S_{z}$. Hence for biological macromolecules, the total evolution of $J+D$ interaction under the operator $2 I_{z} S_{z}$ is conveniently obtained from frequency resolved or intensity modulated heteronuclear multidimensional correlation spectra. A simple strategy for simultaneous measurement of RDC is given in chapter 3. These pulse sequences have been used for proteins studied in this thesis and also for a number of other proteins. 


\section{Chapter 2}

\section{Materials and Methods}

In this chapter, a general description for the materials and methods employed in this work is given.

\section{$2.1 \quad$ Materials}

Protein samples used in the study were mostly prepared by our collaborators or prepared in the molecular biology lab supervised by Dr. Stefan Becker in our department.

\subsubsection{Ubiquitin}

${ }^{15} \mathrm{~N}-{ }^{13} \mathrm{C}$ ubiquitin sample was used in the measurement of residual dipolar couplings for the evaluation of the pulse sequence described in chapter $3 .{ }^{15} \mathrm{~N}-{ }^{13} \mathrm{C}$ ubiquitin was initially bought as a lyophilized powder from VLI research Philadelphia, USA (http://www.vli-research.com). Around $0.8 \mathrm{mM}$ of sample solution was obtained by mixing $1 \mathrm{mg}$ of the lyophilized ubiquitin in $250 \mu \mathrm{l}$ of buffer solution. $50 \mathrm{mM}$ of sodium phosphate buffer containing $95 \% \mathrm{H}_{2} \mathrm{O}$ and $5 \%$ $\mathrm{D}_{2} \mathrm{O}$ at $\mathrm{pH} 6.5$ was used for this study. To obtain anisotropic condition for the measurement of RDCs, lyophilized ubiquitin was directly dissolved into the above mentioned buffer solution, additionally containing 5\% C12E5/n-hexanol $(\mathrm{r}=0.96)$ mixture 38].

\subsubsection{Periplasmic domain of DcuS (DcuS-PD)}

${ }^{15} \mathrm{~N}-{ }^{13} \mathrm{C}$ labeled samples of periplasmic domain of DcuS (DcuS-PD) was produced in the lab of Prof. Gottfried Unden in University of Mainz, Germany. It was expressed in E-coli strain carrying the DcuS $_{45-180}$ plasmid [44]. ${ }^{15} \mathrm{~N}$ labeled mutants namely H110A, F120A and R147A were also produced in the same lab. The buffer condition for the wild type and mutants were 
50 mM Sodium Phosphate at pH 7.0 containing $200 \mathrm{mM} \mathrm{NaCl}, 0.8 \mathrm{mM}$ CHAPS(a detergent), $50 \mathrm{mM}$ Glycine, 50 pM PefablocSC, $0.01 \% \mathrm{NaN}_{3}$ and $\mathrm{H}_{2} \mathrm{O} / \mathrm{D}_{2} \mathrm{O}$ in $90 / 10 \%$. Properly buffered di-sodium salt of Fumaric Acid (Fluka) and D-Tartaric acid (Aldrich) were used for ligand titration. The ligands were dissolved in the same buffer condition as the protein. The pH was adjusted by drop wise addition of $\mathrm{NaOH}(0.1 \mathrm{mM})$ to the buffer containing the ligand. Anisotropic condition for measurement of RDCs for wild type DcuS-PD was obtained by the addition of around $10 \mathrm{mg} / \mathrm{ml}$ of Pf1 phage(Asla) to the protein solution. The quadrapolar splitting of Deuterium was $9.2 \mathrm{~Hz}$.

\subsubsection{Periplasmic domain of CitA (CitAP)}

${ }^{15} \mathrm{~N}$ and ${ }^{15} \mathrm{~N}-{ }^{13} \mathrm{C}$ labeled protein was initially produced in the lab of Prof. Michael Bott in the Institut für Biotechnologie, Forschungszentrum Jülich. ${ }^{15} \mathrm{~N}$ labeled samples were used to optimize the NMR experimental condition for the protein. Two constructs were made: $\mathrm{Cit}_{39-185}$ and $\mathrm{Cit}_{45-176}$. Both the construct had a 6 membered Histidine tag in the $\mathrm{C}$ terminal. Shorter construct, $\mathrm{Cit}_{45-176}$, was used for the assignment experiments as it was more stable, less prone to aggregation and the peaks in the HSQC spectrum of that construct had more favorable line widths (see Chapter 4 Figure 4.18). The NMR buffer was: $50 \mathrm{mM}$ Sodium Phosphate at pH 6.00 containing $\mathrm{H}_{2} \mathrm{O} / \mathrm{D}_{2} \mathrm{O}$ in 90/10\%. Properly buffered tri-sodium citrate di-hydrate(Fluka) and sodium molybdate $\left(\mathrm{Na}_{2} \mathrm{MoO}_{4}\right)$ (Aldrich) were used for ligand titration. These ligands were dissolved in the same NMR buffer. The histidine tag on the protein was cleaved for the double labeled ${ }^{15} \mathrm{~N}-{ }^{13} \mathrm{C}$ CitAP protein. All three dimensional experiments used for the backbone assignments were measured at $298 \mathrm{~K}$. Measurements had to be repeated for the citrate bound form of CitAP.

Later ${ }^{15} \mathrm{~N}-{ }^{13} \mathrm{C}$ labeled CitAP protein was prepared in our molecular biology lab. These samples were more stable than the ones prepared in Jülich. The new double labeled samples were used to re-measure the three dimensional experiments for protein backbone assignment with and without the ligand. Owing to the higher stability, the temperature for the measurements was set to $310 \mathrm{~K}$. The new double labeled samples were also used to measure the RDCs in anisotropic media. Citrate free and citrate bound CitAP samples were aligned separately in 
filamentous bacterial phages (Pf1). Concentration of Pf1 phage in citrate free CitAP sample was $10 \mathrm{mg} / \mathrm{ml}$ and in citrate bound CitAP was $14 \mathrm{mg} / \mathrm{ml}$.

\subsubsection{Equipment}

All NMR spectra were measured in Bruker spectrometers (Bruker Karlsruhe). Following instruments were used to measure the spectra: AVANCE 400, AVANCE 600, DRX 600, AVANCE 700, DRX 800 and AVANCE 900. AVANCE 600, DRX 800 and AVANCE 900 was equipped with a cryo probe for increased signal sensitivity in proton dimension.

\subsubsection{Software}

The software used for processing and analysing NMR spectra, for calculating and analysing structures and for preparing figures is summarized in Table 2.1

\begin{tabular}{|c|c|}
\hline \hline PROGRAM & REFERENCE/ORIGIN \\
\hline DALI & {$[45]$} \\
MARS & {$[46]$} \\
MOLMOL & {$[47]$} \\
MATHEMATICA & Wolfram Research, http://www.wolfram.com \\
NMRPipe/NMRDraw & {$[48]$} \\
PALES & {$[49]$} \\
PyMOL & http://www.pymol.org \\
Sparky 3 & T. D. Goddard and D. G. Kneller, University of California, \\
& San Francisco \\
X-WINNMR 3.5 & Bruker, Karlsruhe, Germany \\
\hline \hline
\end{tabular}

Table 2.1: Table of software used in the study

\subsection{NMR spectroscopy}

Here a brief description of all the methods and the NMR experiments measured are presented. 


\subsubsection{NMR experiments}

All NMR experiments were acquired using Bruker AVANCE 600, 700, and 900 or DRX 600 and 800 spectrometers running under X-WINNMR 3.5. NMR spectra were processed using X-WINNMR 3.5 or NMRPipe/NMRDraw [48] and analysed using Sparky 3 (T. D. Goddard and D. G.Kneller, University of California, San Francisco).

\subsubsection{Chemical shift assignment}

All NMR experiments performed for chemical shift assignments of the different protein samples are summarized in the Tables $2.2,2.3,2.4,2.5$.

\subsubsection{Backbone chemical shift assignment}

Backbone $\mathrm{C}_{\alpha}, \mathrm{C}^{\prime}, \mathrm{N}$ and ${ }^{1} \mathrm{H}^{N}$ assignments were obtained using standard triple resonance experiments used for proteins smaller than $30 \mathrm{kDa}$ [50]. The assignments were, derived from 3D HNCA, CBCA(CO)NH, HNCACB, HNCO and $\mathrm{HN}(\mathrm{CA}) \mathrm{CO}$ experiments. Relaxation optimized experiments like TROSY [51] was also measured for citrate free CitAP protein. The manual assignment of backbone resonances was assisted by using the automatic assignment program MARS [46]. Experiment for the assignments of side chain resonances like 3D HCCH-TOCSY, C(CO)NH-TOCSY and HC(CO)NH-TOCSY spectra were also used for the backbone assignment of CitAP. Refocusing and mixing of ${ }^{13} \mathrm{C}$ magnetization in the HCCHTOCSY experiment was performed using adiabatic pulses [52, 53]. Additional data provided by $3 \mathrm{D}\left[{ }^{15} \mathrm{~N},{ }^{1} \mathrm{H}\right]$-NOESY-HSQC and $\left[{ }^{13} \mathrm{C},{ }^{1} \mathrm{H}\right]$-NOESY-HSQC experiments were used for further assignment as well as confirmation of the through-bond data. For citrate bound form of CitAP, simultaneous ${ }^{13} \mathrm{C},{ }^{15} \mathrm{~N}$ edited NOESY experiment were measured [54, 55]. Following specific experiments were also measured with the citrate free CitAP.

\section{HSQC-CPMG}

It is a ${ }^{15} \mathrm{~N}-{ }^{1} \mathrm{H}$ HSQC experiment designed to reduce chemical exchange broadening of amide proton signals. It was recognized that dephasing of spin by chemical exchange processes can be reduced by CPMG(Carr-Purcell-Meiboom and Gill)-like pulse trains if the rate of the 
$180^{\circ}$ spin-echo is on the order of or exceeds the rate of the exchange process. In this HSQC the INEPT and reverse-INEPT periods for coherence transfer through scalar coupling are modified by the inclusion of space modulated spin echo pulse train [56]. A Bruker pulse sequence (hsqcetf3gpxy.2) was used for the measurement of this experiment.

\section{HACACO}

The three dimensional HACACO experiment was an "out and back" experiment with $\mathrm{H}_{\alpha}$ detection in the direct dimension. This experiment connects the ${ }^{13} C_{\alpha}$ and ${ }^{13} \mathrm{C}^{\prime}$ of the $\mathrm{i}^{\text {th }}$ residue to its $\mathrm{H}_{\alpha} \cdot \mathrm{H}_{\alpha}$ magnetization is first transferred to its one bond coupled ${ }^{13} C_{\alpha}$ using the ${ }^{1} \mathrm{~J}_{\mathrm{C} \alpha H \alpha}$ coupling. The ${ }^{13} C_{\alpha}$ magnetization is transferred to its directly attached ${ }^{13} \mathrm{C}$ ' using the ${ }^{1} \mathrm{~J}_{C \alpha C^{\prime}}$ coupling. After frequency labeling in ${ }^{13} \mathrm{C}^{\prime}$, the magnetization is transferred back to ${ }^{13} C_{\alpha}$. It is frequency labeled in ${ }^{13} C_{\alpha}$ in constant time manner and magnetization is transferred back to directly attached $\mathrm{H}^{\alpha} . \mathrm{H}^{\alpha}$ is detected in the direct dimension. Citrate free CitAP protein was used for the measurement of this pulse sequence. The pulse sequence used was hacacogp3d.vi.

\begin{tabular}{|c|c|c|}
\hline \hline Experiment & Spectrometer & Pulse program \\
\hline 2D ${ }^{15} \mathrm{~N} \mathrm{HSQC}$ & AVANCE 900 cryo probe & hsqc.vi \\
3D HNCA & AVANCE 900 cryo probe & hnca-ge.mz.txt \\
3D HNCACB & AVANCE 900 cryo probe & hncacb-cpd.mz.txt \\
3D CBCA(CO)NH & AVANCE 900 cryo probe & cbcaconh-J.mz \\
\hline \hline
\end{tabular}

Table 2.2: Table of experiments measured for the citrate free CitAP sample that was produced in Jülich

\begin{tabular}{|c|c|c|}
\hline \hline Experiment & Spectrometer & Pulse program \\
\hline 2D ${ }^{15} \mathrm{~N} \mathrm{HSQC}$ & AVANCE 900 cryo probe & hsqc.vi \\
3D HNCO & AVANCE 900 cryo probe & hnco-cpd.fb.vi \\
3D HNCACO & AVANCE 900 cryo probe & hncacogp3d \\
3D HNCACB & AVANCE 900 cryo probe & hncacb-cpd.mz.txt \\
3D CBCA(CO)NH & AVANCE 900 cryo probe & cbcaconh-J.mz \\
\hline
\end{tabular}

Table 2.3: Table of experiments measured for the citrate bound CitAP sample that was produced in Jülich 


\begin{tabular}{|c|c|c|}
\hline Experiment & Spectrometer & Pulse program \\
\hline $2 \mathrm{D}^{15} \mathrm{~N}$ HSQC & AVANCE 900 cryo probe & hsqc.vi \\
\hline $2 \mathrm{D}^{15} \mathrm{~N} \mathrm{HSQ}$ & AVANCE 900 cryo probe & hsqcetf3gpxy.2 \\
\hline 2D ${ }^{13} \mathrm{C}$ HSQC-METHYL & AVANCE 600 cryo probe & ct-hsqc-methyl.vi \\
\hline 3D TROSY-HNCO & AVANCE 600 cryo probe & trhncogp3d \\
\hline $3 \mathrm{D}$ TR & AVANCE & sp3d \\
\hline $3 \mathrm{D}$ & DRX 80 & gp3d \\
\hline $3 \mathrm{D} \mathrm{l}-\mathrm{r} \cdot \mathrm{r} \cdot \mathrm{r}$ & & \\
\hline $3 \mathrm{D} \mathrm{CBCA}(\mathrm{CC}$ & DRX $800 \mathrm{cr}$ & cbcaconhgp3d.vi \\
\hline 3D HC(CO)NH-TOCSY & AVANCE 700 & hccconhgp3d2 \\
\hline $3 \mathrm{D} \mathrm{C}(\mathrm{CC}$ & NCE 700 & hccconl \\
\hline $3 \mathrm{D} \mathrm{HCC}$ & DRX 800 cryo probe & hcchat \\
\hline $3 \mathrm{D}^{15} \mathrm{~N}$ NOESY-HSQC & AVANCE 900 cryo probe & noesyhsqcetf3gp3d \\
\hline 3D ${ }^{13} \mathrm{C}$ NOESY-HSQC & DRX 800 cryo probe & noesyetgp3d13c \\
\hline $2 \mathrm{D}{ }^{1} \mathrm{H}-{ }^{15} \mathrm{~N}$ HetNOE & AVANCE 600 cryo probe & invinoef3gpsi \\
\hline & AVANCE 900 cryo probe & hcacogp3d.vi \\
\hline $2 \mathrm{D}{ }^{1} \mathrm{H}_{-}{ }^{15} \mathrm{~N}$ CCR experiment & DRX 800 cryo probe & hsqcccf3gpphwg.vi \\
\hline
\end{tabular}

Table 2.4: Table of experiments measured for the citrate free CitAP sample that was produced in our laboratory

\begin{tabular}{|c|c|c|}
\hline \hline Experiment & Spectrometer & Pulse program \\
\hline 2D ${ }^{15} \mathrm{~N}$ HSQC & AVANCE 900 cryo probe & hsqc.vi \\
3D HNCO & AVANCE 600 cryo probe & hncogp3d \\
3D HNCA & AVANCE 900 cryo probe & hncagp3d \\
3D HNCACO & AVANCE 600 cryo probe & hncacogp3d.vi \\
3D HNCACB & AVANCE 900 cryo probe & hncacbgp3d \\
3D CBCA(CO)NH & DRX 800 cryo probe & cbcaconhgp3d.vi \\
3D HC(CO)NH-TOCSY & AVANCE 600 cryo probe & hccconhgp3d \\
3D C(CO)NH-TOCSY & AVANCE 600 cryo probe & hccconhgp3d.vi \\
3D HCCH-TOCSY & DRX 800 cryo probe & hcchatgp3d.vi \\
3D ${ }^{15} \mathrm{~N},{ }^{13} \mathrm{C}$ Simultaneous NOESY-HSQC & AVANCE 600 cryo probe & noesyhsqcetf3gp3d \\
2D ${ }^{1} \mathrm{H}^{-15} \mathrm{~N}$ Het-NOE & AVANCE 600 cryo probe & invinoef3gpsi \\
\hline \hline
\end{tabular}

Table 2.5: Table of experiments measured for the citrate bound CitAP sample that was produced in our laboratory 


\subsubsection{Secondary structure determination}

Secondary structure elements in a protein can be identified by calculating the difference between the experimental chemical shift $\left(\mathrm{C}^{\exp }\right)$ and the random coil chemical shift $\left(\mathrm{C}^{\text {coil }}\right)$. Tabulated random coil values were used for folded proteins. Both Schwarzinger and Wishart random coil values were used to derive the secondary chemical shifts [57, 58]. Secondary chemical shifts $(\Delta \delta \mathrm{C})$ for $\mathrm{C} \alpha, \mathrm{C} \beta$ and $\mathrm{C}$ ' were calculated as the difference between measured ${ }^{13} \mathrm{C}$ chemical shift $\left(\delta \mathrm{C}_{\text {exp }}\right)$ and random coil ${ }^{13} \mathrm{C}$ chemical shift $\left(\delta \mathrm{C}_{\text {coil }}\right)$ [59].

$$
\Delta \delta C=\delta C_{\text {exp }}-\delta C_{\text {coil }}
$$

To obtain secondary structure information, the secondary chemical shifts were calculated using the following formula:

$$
\Delta \delta\left(C_{\alpha} C_{\beta} C^{\prime}\right)=\Delta \delta C_{\alpha}-\Delta \delta C_{\beta}+\Delta \delta C^{\prime}
$$

or by adding $\Delta \delta \mathrm{C}_{\alpha}$ and $\Delta \delta \mathrm{C}^{\prime}$ when $\Delta \delta \mathrm{C}_{\beta}$ is not available.

\subsubsection{Residual dipolar couplings (RDCs)}

Alignment media for the measurement of RDCs for individual protein is discussed in the Material part (refer section 2.1). RDCs were back-calculated from X-ray or NMR structures using mostly the order matrix analyis using the singular value decomposition (SVD) method as implemented in PALES [49]. A non linear fit using MATHEMATICA was also used to back calculate RDCs from two or more structures simultaneously. This method was used for the analysis for RDCS of citrate free CitAP.

A description of this method is given below. The RDC analysis described here closely follows the description of the RDCs given in chapter 1, section 1.3. Some of the equations are reproduced here for clarity.

Residual dipolar couplings between spins $\mathrm{m}$ and $\mathrm{n}$ can be expressed as in equation 2.3

$$
D^{m n}=D_{\max }^{m n} \sum_{i j=\{x, y, z\}} S_{i j} \cos \phi_{i}^{n m} \cos \phi_{j}^{n m}
$$


where $\phi_{i}{ }^{n m}$ is the angle of internuclear vector connecting nuclei $n$ and $m$ relative to the $i_{t h}$ molecular axis. $S_{i j}$ are elements of a $3 \times 3$, symmetric and traceless order matrix. Equation 2.3 suggests that if the directional cosines of the internuclear vectors in an arbitrary molecular frame are known, it is possible to determine the order parameters, and hence the molecular orientational properties from just five independent measurements.

Carrying out an order matrix determination mean setting up and solving a system of linear equations of the form $A X=D$.

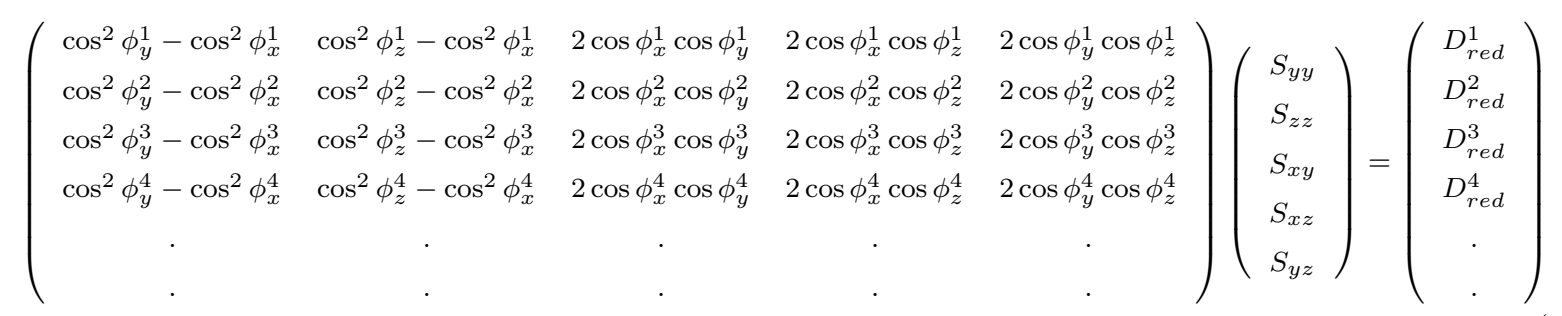

Here $A$ would be the matrix containing the directional cosines obtained from the protein pdb file, $D$ is the column matrix containing the measured RDCs and $X$ would be column matrix containing the elements of the order matrix. Usually the $S_{i j}$ values are obtained by SVD method. This can also be obtained by a fitting program that minimizes the error function $A X-D$ by looking for the best fit parameters for the elements of the order matrix. This is a versatile method used for a simultaneous fitting of two or more structures with their population weighting factor to a measured set of residual dipolar couplings. Here the equation to be solved for the order tensor would be

$$
w_{1} A^{1} X+w_{2} A^{2} X+\ldots+\left(1-\sum_{i}^{n-1} w_{i}\right) A^{n} X=D
$$

Here $A^{1}, A^{2}$ to $A^{n}$ are the directional cosines derived from the 1,2 and $n$th pdb files and $w_{1}, w_{2}$ etc are their population weighting factors. Equation 2.5 was used for the fitting of measured RDCs of citrate free CitAP with different pdb files of CitAP. The fitting procedure was implemented in MATHEMATICA and is given in the appendix. The correlation coefficient and the quality or Q-factor were used to evaluate the agreement between a structure and the observed RDCs. The Q-factor was determined as $\operatorname{rms}\left({ }^{1} \mathrm{D}_{H N}^{\text {measured }}-{ }^{1} \mathrm{D}_{H N}^{\text {calculated }}\right.$ )$/ \operatorname{rms}\left({ }^{1} \mathrm{D}_{H N}^{\text {measured }}\right)$ [60]. A brief description of the type of RDCs measured and the pulse sequence used are given below. 


\section{${ }^{1} \mathbf{H}-{ }^{15} \mathbf{N}$ RDCs}

${ }^{15} \mathrm{~N}-{ }^{1} \mathrm{H}$ splittings were measured under isotropic and anisotropic conditions using 2D IPAP${ }^{15} \mathrm{~N}-{ }^{1} \mathrm{H}$ HSQC experiments [61]. ${ }^{1} \mathrm{H}_{-}{ }^{15} \mathrm{~N}$ RDCs were extracted by subtraction of the ${ }^{1} \mathrm{~J}_{N H}$ scalar coupling measured for the isotropic sample [33]. Alternatively, ${ }^{1} \mathrm{H}^{-15} \mathrm{~N}$ RDCs were obtained from the ${ }^{15} \mathrm{~N}-{ }^{1} \mathrm{H}$ splittings of a modified 3D TROSY-HNCO experiment [62].

\section{C'- ${ }^{15} \mathrm{~N}$ and $\mathrm{C} '-{ }^{13} C_{\alpha}$ RDCs}

The quantitative $\mathrm{J}$ correlation method was used to determine one-bond dipolar ${ }^{13} \mathrm{C}^{\prime}-{ }^{15} \mathrm{~N}$ couplings. Usually this was measured together with the ${ }^{1} \mathrm{H}-{ }^{15} \mathrm{~N}$ RDCs (See Chapter 2) 63] . ${ }^{13} \mathrm{C}^{\prime}{ }^{13} C_{\alpha}$-RDCs were measured from the splitting in the ${ }^{13} \mathrm{C}^{\prime}$ dimension of a ${ }^{13} C_{\alpha}$ coupled 3D HNCO experiment.

\subsubsection{NMR relaxation data}

Characteristic time for a brownian rotation of a molecule is usually called the molecular tumbling time or rotational correlation time $\left(\tau_{c}\right)$. For a spherical molecule of radius $r$ rotating in a solution of viscosity $\eta, \tau_{c}$ is given by

$$
\tau_{c}=\frac{4 \pi r^{3} \eta}{3 k T}=\frac{V \eta}{k T}
$$

where $V$ is the volume of the protein. As $V$ is proportional to molecular mass $\left(V=\rho_{p} \times M\right.$, where $\rho_{p}$ is the partial specific density of the protein written in terms of $1 / \mathrm{g} . \rho_{p}$ is usually $1.39 \times 10^{-3} \mathrm{l} / \mathrm{g}$ for a protein). Taking this value in equation 2.6 and substituting the values for $\eta=0.8872 \times 10^{-3} \mathrm{Ns} / \mathrm{m}^{2}$ for water, and $k=1.3807 \times 10^{-23} \mathrm{~m}^{2} \mathrm{kgs}^{2} \mathrm{~K}^{-1}$ and taking the temperature to be $298 \mathrm{~K}$, we have

$$
\tau_{c} / s=M / k D a \times 0.4459 \times 10^{-9} .
$$

. where $\mathrm{M}$ is the molecular weight of the protein in Kilo-Dalton $(k D a)$. As a rule of thumb the $\tau_{c}$ (in ns) is equal to half the molecular weight of a globular protein expressed in $k D a$.

An approximate value of $\tau_{c}$ can be derived from the equation of $\mathrm{T}_{1 \rho}$ relaxation time for an $\mathrm{N}-\mathrm{H}$ dipolar coupled spins (assuming slow motion limit).

$$
T_{1 \rho}^{-1}=\frac{1}{8}\left(\frac{\mu_{0}}{4 \pi}\right)^{2} \frac{\gamma_{N}^{2} \gamma_{H}^{2} \hbar^{2}}{r^{6}}\{4 J(0)\}
$$


where the spectral density is given by equation 2.12 .

1D $\mathrm{T}_{1 \rho}$ experiments with a spin-lock power of $2.5 \mathrm{kHz}$ was used for the measurement. Two $1 \mathrm{D} \mathrm{T}_{1 \rho}$ experiments were acquired. One with a spin-lock pulse duration of $2 \mathrm{~ms}\left(\Delta_{A}\right)$ and a second with a spin-lock pulse duration $\Delta_{B}$ which corresponds to $\sim 1.3$ times the relaxation time calculated from the theoretical molecular weight. The average relaxation time of all backbone nitrogens was calculated using the following formula:

$$
T_{1 \rho}=\frac{\Delta_{A}-\Delta_{B}}{\ln \left(I_{B} / I_{A}\right)}
$$

\subsection{7 $\quad{ }^{15} \mathrm{~N}-{ }^{1} \mathrm{H}$ steady state NOE}

An important relaxation parameter that is usually measured for biomolecule is the Heteronuclear Nuclear Overhauser Effect (Het-NOE). This is measured by saturating the proton $\left({ }^{1} \mathrm{H}\right)$ signal and observing ${ }^{15} \mathrm{~N}$ signal. The rate at which this occurs is the heteronuclear cross relaxation rate $\mathrm{R}_{H N}\left(\mathrm{H}_{z}<->\mathrm{N}_{z}\right)$; for long proton saturation, it reaches the steady state $\operatorname{NOE}\left(\mathrm{H}_{z}<->\mathrm{N}_{z}\right)$ value. The Het-NOEs of ${ }^{15} \mathrm{~N}$ nuclei in $\mathrm{NH}$ groups were measured using the Bruker standard pulse program invinoef3gpsi. ${ }^{15} \mathrm{~N}-{ }^{1} \mathrm{H}$ Het-NOE values were calculated as the intensity ratios of the ${ }^{15} \mathrm{~N}-{ }^{1} \mathrm{H}$ correlation peaks from pairs of interleaved spectra acquired with and without ${ }^{1} \mathrm{H}$ presaturation during the recycle time of $5 \mathrm{~s}$.

\subsubsection{Measurement of rotational correlation time $\left(\tau_{c}\right)$}

Rotational correlation time for citrate free CitAP was calculated from the cross correlated relaxation term using the NMR relaxation experiment described below. The cross correlated relaxation rate does not have conformational exchange term associated with it. Hence a true value of $\tau_{c}$ can be derived using this experiment. The experiment used here was presented previously for determining the CSA (chemical shift anisotropy) of ${ }^{15} \mathrm{~N}$ spins [64]. Here, the same experiment was used to measure the $\tau_{c}$ for individual residue assuming a constant CSA for ${ }^{15} \mathrm{~N}(160 \mathrm{ppm})$. A brief description of the experiment is given below. The pulse sequence used here is the same as in reference [64].

The pulse sequence 2.1 is essentially an ${ }^{15} \mathrm{~N}-{ }^{1} \mathrm{H}$ HSQC with a relaxation period $2 \Delta$ inserted before the ${ }^{15} \mathrm{~N}$ evolution period. The experiment is performed twice, once with 


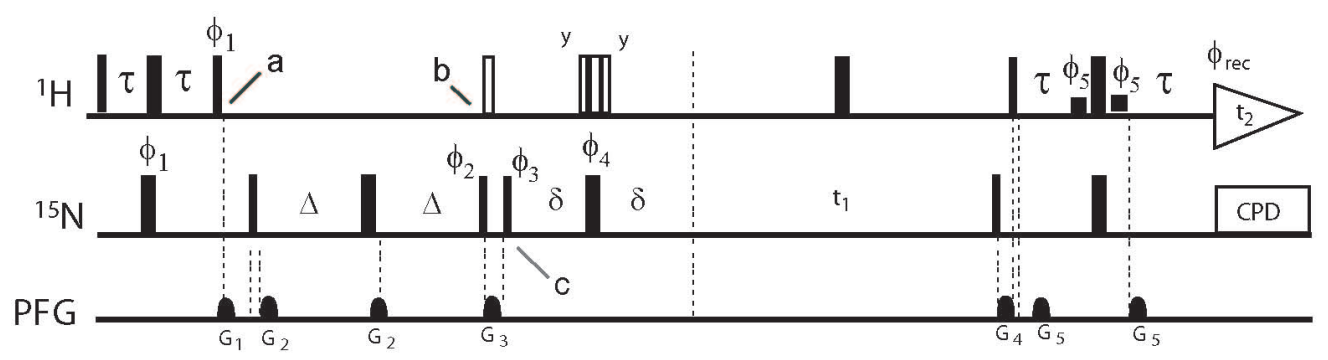

Figure 2.1: Pulse scheme for quantitative measurement of cross correlation. In the reference experiment (scheme B), the open ${ }^{1} \mathrm{H} 90^{\circ}$ and composite $\left(90_{y^{-}} 220_{x^{-}} 90_{y}\right) 180^{\circ}$ pulses are not applied, whereas they are applied in scheme $(\mathrm{A})$, where all resonances are the result of cross correlation effects during the period $2 \Delta$. Narrow and wide pulses correspond to flip angles of $90^{\circ}$ and $180^{\circ}$, respectively. The two low power pulses immediately preceding and following the last non-selective ${ }^{1} \mathrm{H} 180^{\circ}$ pulse have a width of $1 \mathrm{~ms}$ each and correspond to flip angles of $90^{\circ}$. With the carrier positioned on the $\mathrm{H}_{2} \mathrm{O}$ resonance, they are part of the WATERGATE water suppression scheme. The radio-frequency phase of all pulses is assumed $\mathrm{x}$, unless indicated. Delay durations: $\tau \approx$ $2.4 \mathrm{~ms} ; \delta=2.67 \mathrm{~ms}, T_{2} / 8<\Delta<T_{2} / 2$. Phase cycling: $\phi_{1}=y,-y ; \phi_{2}=x, x,-x,-x ; \phi_{4}=$ $4(x), 4(y), 4(-x), 4(-y) ; \phi_{5}=-x$; Receiver $=x, 2(-x), x,-x, 2(x),-x$. Quadrature detection in the $t_{1}$ dimension is accomplished by incrementing $\phi_{3}$ in the States-TPPI manner. All gradients are sine-bell shaped, with an amplitude of $25 \mathrm{G} / \mathrm{cm}$ at their center. Durations: G1,2,3,4,5 = 2.75, 2, 1, 1.5 , and $0.4 \mathrm{~ms}$.

applying the shaded ${ }^{1} \mathrm{H} 90^{\circ}$ and composite ${ }^{1} \mathrm{H} 180^{\circ}$ pulses, and once without applying these pulses. In the first experiment signal is proportional to $\left(\epsilon^{+}-\epsilon^{-}\right) / 2$. In the second experiment signal is proportional to $\left(\epsilon^{+}+\epsilon^{-}\right) / 2$. Here $\epsilon^{ \pm}=\exp [-2 \Delta(\lambda \pm \eta)] . \lambda$ is the auto correlation rate and $\eta$ the cross correlated rate of transverse relaxation of the ${ }^{15} \mathrm{~N}$ spin. The ratio of signal intensities obtained in the two experiment will then be

$$
I_{A} / I_{B}=\left(\epsilon^{-}-\epsilon^{+}\right) /\left(\epsilon^{-}+\epsilon^{+}\right)=\tanh (2 \Delta \eta)
$$

where

$$
\eta=2 \alpha d\left\{4 J(0)+3 J\left(\omega_{N}\right)\right\}\left(3 \cos ^{2} \theta-1\right) / 2
$$

with $\alpha=\frac{\mu_{0} \gamma_{I} \gamma_{S} h}{16 \sqrt{2} \pi^{2} r^{3}}$ and $d=\frac{\gamma_{S} B_{0} \Delta \sigma}{3 \sqrt{2}}$

For a spherical rigid molecule

$$
J(\omega)=\frac{2}{5} \frac{\tau_{c}}{\left(1+\omega^{2} \tau_{c}^{2}\right)}
$$


For slow motion limit $\left(\omega \tau_{c}>>1\right)$, the term $J(\omega)$ can be neglected. Hence only $J(0)$ contribute to the cross relaxation term. Substituting $\alpha, d$ and taking only the $J(0)$ term for the spectral density Equation 2.11 becomes

$$
\eta=\frac{\mu_{0} \gamma_{I} \gamma_{S}^{2} h B_{0}(\Delta \sigma) \tau_{c}\left(3 \cos ^{2} \theta-1\right)}{240 \pi^{2} r^{3}}
$$

Substitution of equation 2.13 to equation 2.10 and fitting that equation to signal ratios measured at varying $\Delta$ gives a value for $\tau_{c}$. Values of the physical constants are compiled in the table 2.6 ,

\begin{tabular}{|c|c|}
\hline \hline Symbol & Values \\
\hline$\gamma\left({ }^{1} \mathrm{H}\right)$ & $26.7519^{*} 10^{7} \mathrm{~s} \mathrm{~A} \mathrm{~kg}^{-1}$ \\
$\gamma\left({ }^{15} \mathrm{~N}\right)$ & $-2.7519^{*} 10^{7} \mathrm{~s} \mathrm{~A} \mathrm{~kg}^{-1}$ \\
$\mathrm{~h}$ (plancks constant) & $6.623^{*} 10^{-34} \mathrm{~J} \mathrm{~s}^{-2}$ \\
$\mu_{0}$ (permeability of vaccum) & $4^{*} \pi^{*} 10^{-7} \mathrm{~m} \mathrm{~kg} \mathrm{~s}^{-2} \mathrm{~A}^{-2}$ \\
$\mathrm{r}$ (distance of N-H bond) & $1.04^{*} 1010^{-10} \mathrm{~m}$ \\
$\mathrm{~B}$ (field strength) & $800.25 \mathrm{MHz}$ \\
\hline \hline
\end{tabular}

Table 2.6: Compilation of physical constants

For citrate free CitAP, ratios of intensities were obtained for two delays ( $\Delta=16$ and $48 \mathrm{~ms}$ ). These ratios were fitted against the equation in 2.10. The slow motion limit and spectral density for rigid spherical rotor was used.

The value of $\tau_{c}$ obtained using this method does not take into consideration the local motions associated with the spins. Hence a lower bound for the $\tau_{c}$ estimate is obtained using the spectral density function based on rigid spherical model. More general spectral density function has been proposed by Lipari and Szabo [65, 66, 67] which is the sum of two Lorentian shapes.

$$
J(\omega)=\frac{2}{5}\left[S^{2} \frac{\tau_{m}}{1+\left(\omega \tau_{m}\right)^{2}}+\left(1-S^{2}\right) \frac{\tau}{1+(\omega \tau)^{2}}\right]
$$

with $\tau^{-1}=\tau_{m}^{-1}+\tau_{e}^{-1}$. Here the relaxation rate depends on one global parameter $\left(\tau_{m}\right)$ which is same for all residues and two local parameters, $\tau_{e}$, a correlation time for internal motions and $S^{2}$, which is a measure of amplitude of internal motion, known as the order parameter. Usually $\tau_{e}$ is very small (order of ps) and hence the second term in the equation 2.14 can be neglected. This truncated spectral density function is scaled by a factor of $S^{2}$ 
with respect to the spectral density used for the derivation of $\tau_{c}$. Common values of order parameter for a globular medium size proteins range from $0.79-0.85$. Hence the estimation of the $\tau_{c}$ using spectral density in equation 2.12 underestimates the values by $\sim 20 \%$. But for residues showing considerable low values of $\tau_{c}$, the average would then indicate considerable contribution from internal motions (order parameter low or $S^{2}<<0.8$ ).

\subsubsection{Chemical shift mapping of binding surfaces}

An easy and rapid way to gain qualitative information about the interaction between two molecules is to study the change in chemical shift values. Perturbation in the local environment of the nucleus have a great effects on the chemical shift of that nucleus. The chemical shifts of ${ }^{1} \mathrm{H}$ and ${ }^{15} \mathrm{~N}$ are particularly sensitive to changes of the chemical environment. Therefore ${ }^{15} \mathrm{~N}$ and ${ }^{1} \mathrm{H}$ chemical shift changes recorded via ${ }^{15} \mathrm{~N}-{ }^{1} \mathrm{H}-\mathrm{HSQC}$ spectra throughout an NMR titration are commonly combined in order to map a binding site on a protein according to the following equation

$$
\Delta \delta_{H N}=\sqrt{\frac{\left(\Delta \delta_{N} / 5\right)^{2}+\left(\Delta \delta_{H}\right)^{2}}{2}}
$$

$\Delta \delta_{H N}$ : average amide chemical shift change.

$\Delta \delta_{N}$ : amide nitrogen chemical shift change.

$\Delta \delta_{H}$ : amide proton chemical shift change.

The binding interface is defined by the residues exhibiting the largest ${ }^{15} \mathrm{~N}$ and ${ }^{1} \mathrm{H}$ chemical shift changes upon the NMR titration. 


\section{Chapter 3}

\section{Simultaneous measurement of protein one-bond residual dipolar couplings without increased resonance overlap}

\subsection{Abstract}

A NMR strategy designed to measure simultaneously and without increased resonance overlap scalar and dipolar couplings (RDCs) in ${ }^{13} \mathrm{C}-{ }^{15} \mathrm{~N}$-labeled proteins is presented. Contrary to common schemes for simultaneous measurement of RDCs, a single reference experiment is used for the extraction of more than one type of coupling, thereby reducing the required measurement time. This is accomplished by a common reference spectrum followed by a series of interleaved experiments, in which a particular coupling dependent parameter is varied according to the quantitative $J$-correlation method or using accordion spectroscopy. To illustrate this idea, we have modified the 3D TROSY-HNCO and the $3 \mathrm{D} \mathrm{CBCA}(\mathrm{CO}) \mathrm{NH}$ experiment allowing efficient measurement of one-bond ${ }^{1} \mathrm{D}_{N H},{ }^{1} \mathrm{D}_{\mathrm{C} \alpha \alpha}$, ${ }^{1} \mathrm{D}_{C^{\prime} N},{ }^{1} \mathrm{D}_{C \beta H \beta}$ and ${ }^{1} \mathrm{D}_{C \alpha C^{\prime}}$ couplings in small to medium sized proteins. In addition, the experiments are expected to be useful for largely unfolded proteins, which show strong resonance overlap but have very favorable relaxation properties. Measurement of RDCs is demonstrated on uniformly ${ }^{15} \mathrm{~N}-{ }^{13} \mathrm{C}$-labeled ubiquitin and on the sensory domain of the membraneous two-component fumarate sensor DcuS of Escherichia coli $(17 \mathrm{kDa})$. DcuS was found to be unstable and to precipitate in one to two weeks. RDCs obtained from these experiments are in good agreement with the $1.8 \AA \mathrm{X}$-ray structure of ubiquitin. 


\subsection{Introduction}

Residual dipolar couplings (RDCs) can be observed in solution when a molecule is aligned with the magnetic field, either as a result of its own magnetic susceptibility anisotropy [68, 69] or caused by an anisotropic environment such as an oriented liquid crystalline phase [70] or an anisotropically compressed gel [39, 71]. When an alignment can be kept sufficiently weak, the NMR spectra retain the simplicity normally observed in regular isotropic solution, while allowing quantitative measurement of a wide variety of RDCs, even in macromolecules [33, 32]. In proteins, the most commonly measured dipolar couplings are the one bond ${ }^{1} \mathrm{D}_{N H},{ }^{1} \mathrm{D}_{C \alpha H \alpha},{ }^{1} \mathrm{D}_{C^{\prime} N}$, and ${ }^{1} \mathrm{D}_{C \alpha C^{\prime}}$ RDCs. These couplings are sufficiently large to be measured with good accuracy, allow definition of the orientation of a peptide plane and play a key role in RDC-based structure determination [46, 72, 73, 74].

However, it is observed that certain proteins are unstable under the orienting media condition. If the magnitude and/or the orientation of the alignment tensor are changing slowly over time due to instability of the sample (protein or liquid crystal), the different sets of dipolar couplings will not be consistent with a unique alignment tensor/structure. This will in turn bias the protein structure calculation process. Therefore, it is desirable to measure as many types of RDCs in the committed time in which the protein/liquid crystal is stable. Moreover, much focus is put on dipolar couplings for rapid structure determination, with the aim to establish NMR spectroscopy as an important tool in Structural Genomics 75. Therefore, there is the need to measure a large set of dipolar couplings in an as short time as possible.

Instead of measuring one type of coupling per NMR experiment, a number of schemes have been proposed recently that allow simultaneous measurement of different types of dipolar couplings in a single spectrum: ${ }^{1} \mathrm{D}_{H N} /{ }^{1} \mathrm{D}_{C \alpha C^{\prime}}$ or ${ }^{1} \mathrm{D}_{H N} /{ }^{1} \mathrm{D}_{C \alpha H \alpha}$ from frequency differences using the IPAP-strategy [61, 76], ${ }^{1} \mathrm{D}_{C \alpha C^{\prime}} /{ }^{3} \mathrm{D}_{H N C \alpha}$ or ${ }^{1} \mathrm{D}_{N C \alpha} /{ }^{2} \mathrm{D}_{N C \alpha} /{ }^{2} \mathrm{D}_{H N C \alpha} /{ }^{3} \mathrm{D}_{H N C \alpha}$ from 3D $\operatorname{HNCO}(\alpha / \beta$-C'C $\alpha$ - $J)$-TROSY and $\operatorname{HNCO}(\alpha / \beta$-NC $\alpha-J)$-TROSY [77], ${ }^{1} D_{N C \alpha} /{ }^{2} D_{H N C \alpha}$ from ${ }^{13} C^{\alpha}$ coupled or $J$-modulated $\left[{ }^{15} \mathrm{~N},{ }^{1} \mathrm{H}\right]$-HSQC spectra [78], ${ }^{1} D_{C^{\prime} N} /{ }^{1} D_{N C \alpha} /{ }^{2} D_{N C \alpha}$ from a quantitative $J$-correlation 3D TROSY-HNC experiment, in which cross and reference axial 
peaks are observed in a single spectrum [79], ${ }^{1} D_{C \alpha H \alpha} /{ }^{2} D_{N H \alpha}$ from HNCA-TROSY [80], and ${ }^{1} \mathrm{D}_{C^{\prime} N} /{ }^{2} \mathrm{D}_{H N C^{\prime}}$ from an E.COSY-type HSQC experiment [81]. Approaches in which multiple couplings are allowed to evolve in a single experiment, have the disadvantage that each coupling results in a splitting that reduces the signal intensity by one half. Therefore, the overall sensitivity of these experiments is not higher than when the couplings are measured sequentially (potentially even lower if not multiple values for each $J+\mathrm{D}$ value are obtained in the same experiment). In addition, if the multiplet components are not separated in different spectra this will result in increased overlap. Increased overlap is also a problem for the quantitative $J$-correlation TROSY-HNCO experiment, as reference peaks are located in a single plane of the 3D spectrum and both $\mathrm{C}^{\alpha}(\mathrm{i})$ and $\mathrm{C}^{\alpha}(\mathrm{i}-1)$ peaks are present [79].

Here we present a different strategy for simultaneous measurement of dipolar couplings that is based on the quantitative J-correlation method and accordion spectroscopy. Only a single coupling is active during a single experiment, but for different couplings the same reference spectrum is used. This can reduce the total required measurement time without increasing resonance overlap. In addition, the experiments, in which different couplings are active, are recorded interleaved, such that measured RDCs correspond to a unique alignment tensor. In order to illustrate this concept, we have modified the 3D experiments TROSY$\mathrm{HNCO}$ and $\mathrm{CBCA}(\mathrm{CO}) \mathrm{NH}$ for efficient measurement of ${ }^{1} \mathrm{D}_{N H},{ }^{1} \mathrm{D}_{C \alpha H \alpha},{ }^{1} \mathrm{D}_{C N},{ }^{1} \mathrm{D}_{C \beta H \beta}$ and ${ }^{1} \mathrm{D}_{C \alpha C}$ couplings in small to medium sized, globular or larger, but intrinsically unstructured proteins.

\subsection{Description of the pulse sequences}

All presented pulse sequences can be used for measurement of one-bond isotropic scalar couplings. Dipolar couplings are obtained by performing the experiments both in an isotropic and a partially aligned phase followed by subtraction of the two measured values. 


\subsubsection{TROSY-HNCO experiment}

The pulse sequence of the 3D TROSY-HNCO experiment for simultaneous measurement of ${ }^{1} \mathrm{D}_{C N}$ and ${ }^{1} \mathrm{D}_{N H}$ couplings is shown in Figure 3.1. It consists of three interleaved experiments that are based on the 3D TROSY-HNCO [51].

The backbone ${ }^{1} J_{C^{\prime} N}$ coupling is obtained by the quantitative $J$-correlation approach [62. A reference experiment is recorded in which peak intensities are not strongly affected by the coupling of interest. This is achieved with the $180^{\circ}$ pulse at position (a) and with a conventional constant time ${ }^{15}$ Nevolution period as depicted in panel (i). The ${ }^{15} \mathrm{~N}-{ }^{13} \mathrm{C}$ ' dephasing delay is adjusted to $2 \mathrm{~T}=66.7 \mathrm{~ms}$ and the separation of the $180^{\circ}$ pulses amounts to $\Delta=16.7 \mathrm{~ms}$. Thus, the effective dephasing time due to ${ }^{15} \mathrm{~N}-{ }^{13} \mathrm{C}$ 'couplings is $2 T-2 \Delta=$ $33.3 m s$, i.e., close to $1 /\left(2^{1} \mathrm{~J}_{C^{\prime} N}\right)$. To attenuate cross peak intensities according to the ${ }^{1} \mathrm{~J}_{C^{\prime} N}$ coupling, the $180^{\circ}$ inversion pulse on $\mathrm{C}^{\prime}$ is shifted to position (b) and the ${ }^{1} \mathrm{~J}_{C^{\prime} N}$ coupling is active for the entire interval. ${ }^{1} \mathrm{~J}_{C^{\prime} N}$ can then be calculated from the intensity ratios of the reference, $I_{r e f}$, and attenuated resonances, $I_{a t t}$, according to

$$
\frac{I_{a t t}}{I_{\text {ref }}}=\frac{\sin \left(2 \pi^{1} J_{C^{\prime} N} T\right)}{\sin \left[2 \pi^{1} J_{C^{\prime} N}(T-\Delta)\right]}
$$

This previously described approach for measurement of ${ }^{1} \mathrm{~J}_{C^{\prime} N} 62$ is extended by addition of a third experiment, which is recorded in an interleaved manner with the two described above, and allows simultaneous measurement of ${ }^{1} \mathrm{~J}_{N H}$ couplings. The $180^{\circ}$ inversion pulse on the carbonyls is kept at position (a), in order to maximize the ${ }^{15} \mathrm{~N}-\left\{{ }^{13} \mathrm{C}\right.$ ' $\}$ transfer amplitude. Opposite to the reference spectrum, however, an additional delay is inserted at the end of the constant time ${ }^{15} \mathrm{~N}$ evolution period that is incremented together with the ${ }^{15} \mathrm{~N}$ evolution time (Figure 3.1 panel (ii)) [82]. During this delay, the ${ }^{15} \mathrm{~N}-\left\{{ }^{1} \mathrm{H}\right\}$ coupling evolves with respect to its directly attached proton while chemical shift evolution is refocused. Therefore, the peaks appear at $\omega N-(\kappa+1) \pi^{1} J_{N H}$ rather than at $\omega N-\pi^{1} J_{N H}$ as in the reference spectrum with ${ }^{1} J_{N H}<0$. Typically $\kappa$ is set to 2 so that a particular cross peak is shifted between the spectra by ${ }^{1} \mathrm{~J}_{\mathrm{NH}} \mathrm{Hz}$ in the ${ }^{15} \mathrm{~N}$ dimension. In this way, a common reference experiment (Figure 3.1 . a, (i)) can be used for extraction of ${ }^{1} \mathrm{~J}_{C^{\prime} N}$ (Figure 3.1. b, (i)) and ${ }^{1} \mathrm{~J}_{N H}$ (Figure 3.1, a, (ii)). Note that the two $180^{\circ}$ pulses on ${ }^{1} \mathrm{H}$ and ${ }^{15} \mathrm{~N}$, which are necessary for measurement of the 
${ }^{1} \mathrm{~J}_{N H}$ coupling, are also present in the reference and the ${ }^{1} \mathrm{~J}_{C^{\prime} N}$-attenuated experiment, to have the same number and type of pulses in all experiments.

\subsection{2 $\mathrm{CBCA}(\mathrm{CO}) \mathrm{NH}$ experiment}

One-bond ${ }^{1} \mathrm{D}_{C \alpha H \alpha},{ }^{1} \mathrm{D}_{C \beta H \beta}$, and ${ }^{1} \mathrm{D}_{C \alpha C^{\prime}}$ dipolar couplings are measured with a modified version of the $3 \mathrm{D} \mathrm{CBCA}(\mathrm{CO}) \mathrm{NH}$ pulse sequence, which has been enhanced by the use of adiabatic pulses [53, 83]. The experiment is performed four times in an interleaved manner (Figure 3.2). All couplings are obtained using the quantitative $J$-correlation technique. The ${ }^{1} \mathrm{~J}_{C \alpha H \alpha}$ and ${ }^{1} \mathrm{~J}_{C \beta H \beta}$ couplings are obtained from the first three interleaved experiments, in which the ${ }^{1} \mathrm{H} 180^{\circ}$ pulse during the $t_{1}$ evolution period is switched between positions a, $\mathrm{b}$ and c, respectively [84]. The rephasing of the ${ }^{1} \mathrm{~J}_{C H}$ coupling of $\mathrm{C}_{\beta}$ and $\mathrm{C}_{\alpha}$ at the end of the carbon constant time period depends on the duration of $\Delta_{1}$. This is reflected in the spectra by a modulation of signal intensities according to

$$
S\left(\Delta_{1}\right) \sim \sin \left[\pi \sum J_{C H}\left(2 \Delta_{1}+\Delta_{e f f}\right)\right]
$$

where $\Delta_{\text {eff }}$ accounts for the effect of ${ }^{1} \mathrm{~J}_{C H}$ dephasing during the adiabatic ${ }^{13} \mathrm{C}$ inversion pulse, during the ${ }^{13} \mathrm{C} 90^{\circ}$ pulses, and during short switching delays, and from which $\sum \mathrm{J}_{C H}$ can be derived by a SIMPLEX minimization routine.

${ }^{1} \mathrm{~J}_{C \alpha C^{\prime}}$ couplings are obtained from the first experiment (this is also used as a reference spectrum for the measurement of ${ }^{1} \mathrm{~J}_{C \alpha H \alpha}$ and ${ }^{1} \mathrm{~J}_{C \beta H \beta}$ couplings) and an additional fourth, interleaved experiment. In order to attenuate signal intensities according to the one-bond ${ }^{1} \mathrm{~J}_{C \alpha C^{\prime}}$ values, the $\mathrm{C}^{\prime}$ to ${ }^{15} \mathrm{~N}$ transfer step is used (Figure 3.2. In all four interleaved experiments, the total dephasing delay is set to $2(n+\zeta)=22 \mathrm{~ms}$. In the regular CBCA $(\mathrm{CO}) \mathrm{NH}$ experiment (i.e. the reference experiment) a $180^{\circ}$ inversion pulse is applied a duration $\zeta$ after the $90^{\circ}$ excitation pulse on $\mathrm{C}^{\prime}$, such that the ${ }^{1} \mathrm{~J}_{C \alpha C^{\prime}}$ coupling is active for $2 \zeta=9 \mathrm{~ms}$, i.e., close to $1 /\left(2^{1} \mathrm{~J}_{C^{\prime} N}\right)$. In the fourth interleaved experiment, however, the open $180^{\circ}$ pulse on $\mathrm{C}_{\alpha}$ is shifted by a duration $\Delta$ closer to the $180^{\circ} \mathrm{C}^{\prime}$ pulse making ${ }^{1} \mathrm{~J}_{C \alpha C^{\prime}}$ active for $2(\zeta+\Delta)=18.7 m s^{1} \mathrm{~J}_{C \alpha C^{\prime}}$ couplings are almost rephased at the end of the $\mathrm{C}^{\prime}$ to ${ }^{15} \mathrm{~N}$ transfer step, yielding weak resonance intensity. The ratio of signal intensities from the reference and 

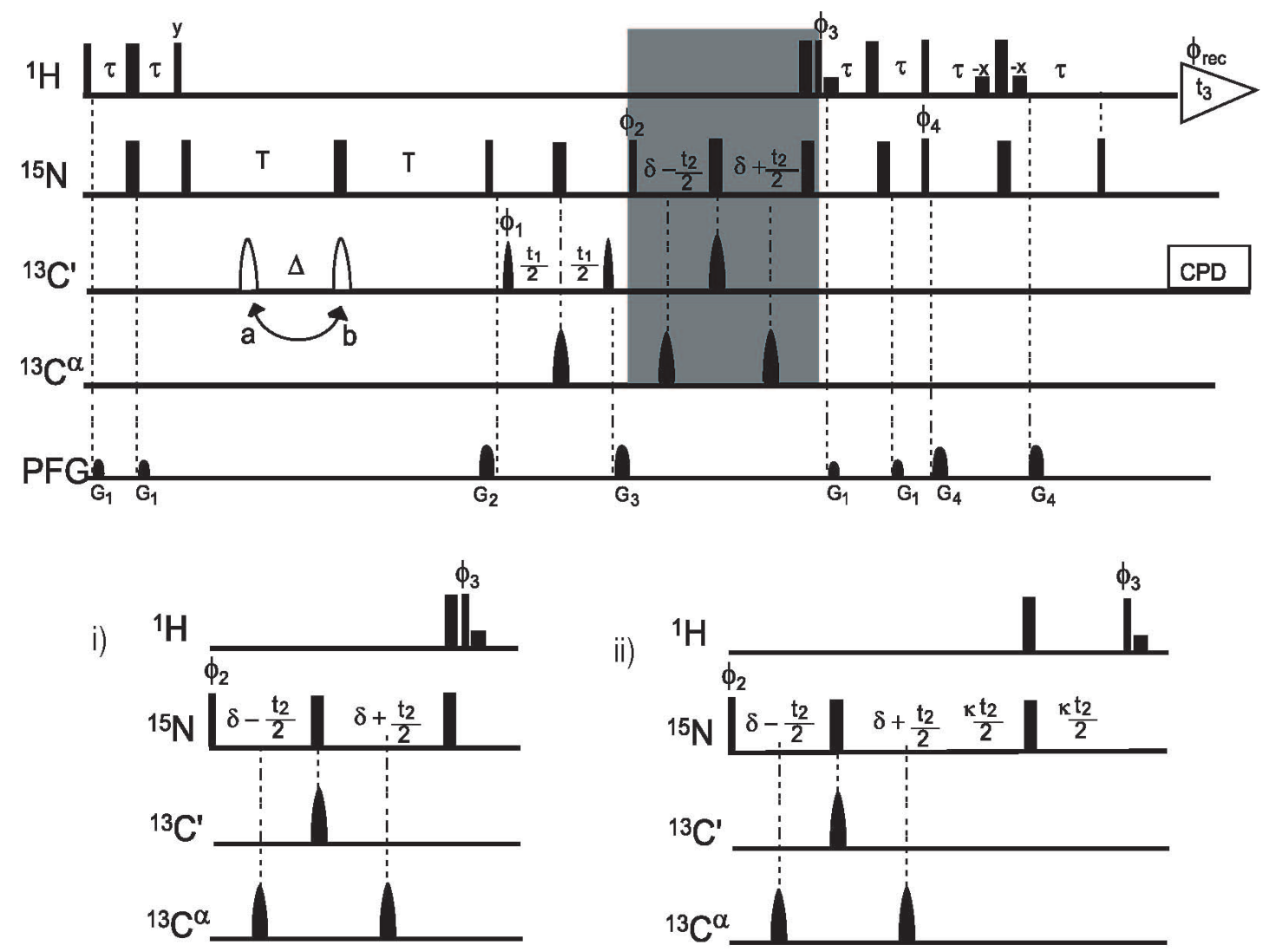

Figure 3.1: Pulse scheme of the modified 3D TROSY-HNCO experiment for simultaneous measurement of ${ }^{1} \mathrm{~J}_{C^{\prime} N}$ and ${ }^{1} J_{N H}$ couplings. Narrow and wide pulses correspond to flip angles of $90^{\circ}$ and $180^{\circ}$ respectively. All pulse phases are $\mathrm{x}$, unless specified otherwise. ${ }^{13} \mathrm{C}$ ' pulses bracketing the $t_{1}$ evolution period are $90^{\circ}$ and have the shape of the center lobe of a $\sin x / x$ function, and durations of $120 \mu \mathrm{s}$ (at $176 \mathrm{MHz}{ }^{13} \mathrm{C}$ frequency). The other two ${ }^{13} \mathrm{C}^{\prime}$ pulses are hyperbolic secant shaped (1 ms) and correspond to $180^{\circ}$. Composite pulse ${ }^{13} \mathrm{C}^{\prime}$ decoupling (CPD) was used during acquisition. All three $180^{\circ}{ }^{13} \mathrm{C}_{\alpha}$ pulses are sine-bell shaped and have durations of $150 \mu \mathrm{s}$. Delay durations: $\tau=2.5 \mathrm{~ms} ; \mathrm{T}=33.7 \mathrm{~ms} ; \delta=16 \mathrm{~ms} ; \Delta=16.6 \mathrm{~ms}$. For the reference and the ${ }^{1} J_{N H}$-shifted spectrum, the first $180^{\circ}{ }^{13} \mathrm{C}^{\prime}$ pulse (open shape) is applied in position a; for the rephased, ${ }^{1} J_{C^{\prime} N}$ -attenuated spectrum in position b. The shaded portion of the pulse scheme is given in detail in (i) and (ii). (i) Used for the first and second interleaved spectrum and (ii) used for the third interleaved spectrum for measurement of ${ }^{1} \mathrm{~J}_{N H}$ couplings. In the shifted spectrum (ii) $\kappa$ is typically set to 2 , to obtain a shift of ${ }^{1} J_{N H} \mathrm{~Hz}$ in the ${ }^{15} \mathrm{~N}$ dimension. Two FIDs are acquired and stored separately for obtaining quadrature selection of the TROSY component in the $t_{2}$ dimension, with phases $\phi_{2}$ $=y, x, \phi_{3}=-y, \phi_{4}=-y$ and with phases $\phi_{2}=y,-x, \phi_{3}=y, \phi_{4}=y$ [85. In both cases, $\phi_{1}=x$ $, x,-x,-x$, and Receiver $=x, y,-x,-y$. States-TPPI quadrature selection is used in the $\mathrm{t}_{1}$ dimension. Sine-bell shaped pulsed field gradients (1 ms each) have strength $(\mathrm{G} / \mathrm{cm})$ and axis: $\mathrm{G} 1: 3, x ; \mathrm{G} 2$ : 18, $y$; G3: 18, $x$; G4: 18, z. 


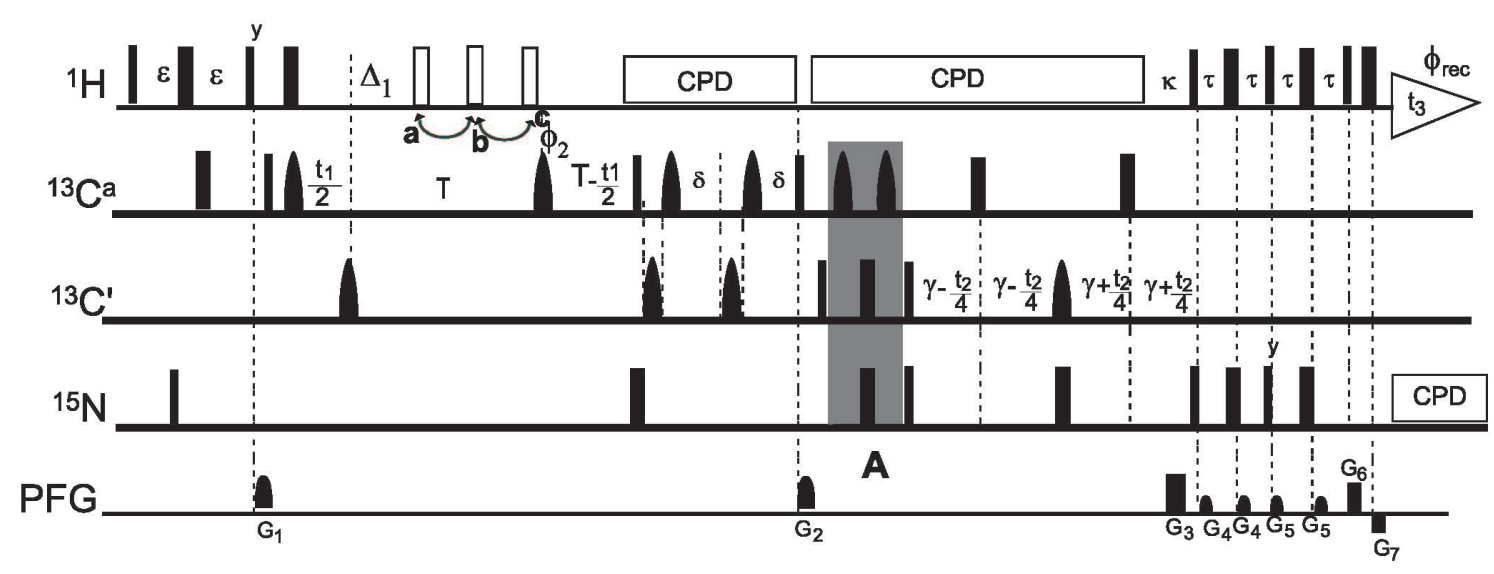

A

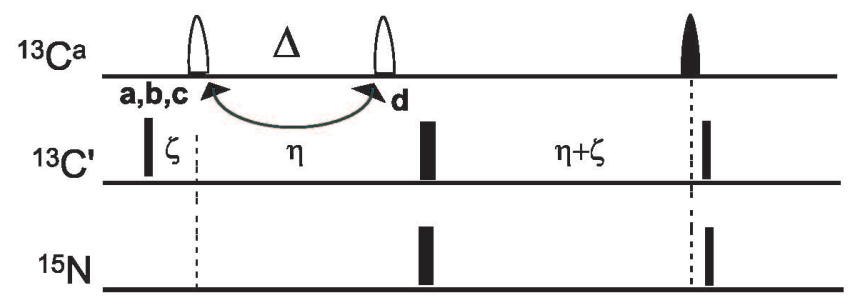

Figure 3.2: Pulse scheme of the 3D CBCA(CO)NH quantitative $J_{C H}$ and $J_{C \alpha C^{\prime}}$ experiment. Narrow and wide pulses correspond to flip angles of $90^{\circ}$ and $180^{\circ}$ respectively. All pulse phases are $x$, unless specified otherwise. The first three ${ }^{13} \mathrm{C}^{\prime} 180^{\circ}$ pulse has rSNOB profile [86, and a duration of $170 \mu \mathrm{s}$ (at $150 \mathrm{MHz} 13 \mathrm{C}$ frequency). All other shaped ${ }^{13} \mathrm{C}^{\prime}$ and ${ }^{13} \mathrm{C}_{\alpha / \beta}$ pulses (46 ppm) are of the hyperbolic secant adiabatic inversion type $(500 \mu \mathrm{s})$, with a squareness level, $\mu$, of 3 [87. The open ${ }^{1} \mathrm{H}$ pulses, applied at time point a, b, or c, is composite $\left(90^{\circ} x^{-} 180^{\circ} y^{-} 90^{\circ} x\right)$. Delay durations: $\epsilon=1.3 \mathrm{~ms} ; \Delta_{1}=0.77,1.67$ and $3.42 \mathrm{~ms}$ in the three interleaved experiments; $\mathrm{T}=3.4 \mathrm{~ms} ; \delta=3.4$ $\mathrm{ms} ; \eta=6.5 \mathrm{~ms} ; \gamma=6.8 \mathrm{~ms} ; \kappa=5.4 \mathrm{~ms} ; \tau=2.7 \mathrm{~ms}$. For the reference spectrum, the first $180^{\circ 1} \mathrm{H}$ pulse (open shape) is applied at position a, which gives a total $J_{C H}$-modulation duration of $2\left(\Delta_{1}\right.$ $\left.+\Delta_{\text {eff }}\right) 1.93 \mathrm{~ms}$ [84]. In the $J_{C \alpha C^{\prime}}$-attenuated experiment (the fourth interleaved spectrum) the open ${ }^{13} \mathrm{C} 180^{\circ}$ pulse is applied at point $\mathrm{d}$, otherwise at position a/b/c. Delay durations: $\Delta=4.85$ $\mathrm{ms} ; \xi=4.5 \mathrm{~ms}$. Note that the second $180^{\circ}$ pulse on $\mathrm{C}_{\alpha}$ compensates the Block - Siegert shift, and should be applied with a reverse phase profile in order to refocus the coupling evolution during the open ${ }^{13} \mathrm{C} 180^{\circ}$ pulse [88]. Phase cycling: $\phi_{1}=y,-y ; \phi_{2}=x, x, y, y$; Receiver $=x,-x,-x, x$. Rance-Kay $\mathrm{t}_{2}$ quadrature detection is used, [26] alternating the phase of the first ${ }^{15} \mathrm{~N} 90^{\circ}$ pulse after gradient $\mathrm{G}_{3}$ between $x$ and $-x$, in concert with the polarity of gradient $\mathrm{G}_{3}$. States-TPPI quadrature selection is used in the $t_{1}$ dimension. Pulsed field gradients $\mathrm{G}_{1,2,4,5}$ are sine-bell shaped (18 $\mathrm{G} / \mathrm{cm}$ for $\mathrm{G}_{1,2}, 28$ $\mathrm{G} / \mathrm{cm}$ for $\left.\mathrm{G}_{4,5}\right)$, and $\mathrm{G}_{3,6,7}$ are rectangular $(30 \mathrm{G} / \mathrm{cm})$. Durations: $\mathrm{G}_{1,2,3,4,5,6,7}=2,1.4,2.705,0.4$, $0.4,0.2,0.074 \mathrm{~ms}$, with respective gradient axes: $x y, y, z, x, y, z, z$. 
attenuated spectrum follow a similar relationship as Eq. [1], from which ${ }^{1} \mathrm{~J}_{C \alpha C^{\prime}}$ couplings can be extracted by a one dimensional grid search or a nonlinear fit. Note that the $\mathrm{C}_{\beta}$ resonances are also modulated by the ${ }^{1} \mathrm{~J}_{C \alpha C^{\prime}}$ coupling. Thus, if there is signal overlap in the $\mathrm{C}_{\alpha}$ region of the spectrum one can still obtain the couplings from the $\mathrm{C}_{\beta}$ resonances, provided the particular residues do not have overlapping $\mathrm{C}_{\beta}$ frequencies.

3D $\mathrm{CBCA}(\mathrm{CO}) \mathrm{NH}$ and TROSY-HNCO are among the most sensitive triple resonance experiments for medium sized proteins and the method is applicable to any protein which yields TROSY-HNCO and CBCA(CO)NH spectra with high $\mathrm{S} / \mathrm{N}(\geq 20: 1$ and $\geq 40: 1$, respectively) in a reasonable amount of time.

\subsection{Data collection}

The method is demonstrated for uniformly ${ }^{13} \mathrm{C} /{ }^{15} \mathrm{~N}$-labeled human ubiquitin (VLI Research Inc., Philadelphia) and on the uniformly ${ }^{13} \mathrm{C} /{ }^{15} \mathrm{~N}$-labeled sensory domain of the membraneous two-component fumarate sensor DcuS of E. coli $(17 \mathrm{kD})$ [44]. One-bond couplings were measured for an isotropic sample, containing $0.8 \mathrm{mM}$ ubiquitin in $95 \% \mathrm{H} 2 \mathrm{O}$, $5 \%$ D2O, $50 \mathrm{mM}$ phosphate buffer, $\mathrm{pH} 6.5$ at $35{ }^{\circ} \mathrm{C}$, or containing $0.8 \mathrm{mM}$ DcuS in $95 \%$ $\mathrm{H} 2 \mathrm{O}, 5 \%$ D2O, $50 \mathrm{mM}$ phosphate buffer, $\mathrm{pH} 6.5$ at $30{ }^{\circ} \mathrm{C}$, and a second sample containing additionally either $5 \% \mathrm{C} 12 \mathrm{E} 5 / \mathrm{n}$-hexanol $(\mathrm{r}=0.96)$ (in case of ubiquitin) [38] or $10 \mathrm{mg} / \mathrm{ml}$ bacteriophage Pf1 (in case of DcuS) [37, 89].

Spectra were recorded on BRUKER AVANCE 600 and $700 \mathrm{MHz}$ spectrometers. TROSYHNCO experiments were acquired as $24^{*} \times 40^{*} \times 512^{*}$ data matrices with acquisition times of $16.8 \mathrm{~ms}\left(t_{1},{ }^{13} \mathrm{C}^{\prime}\right), 18.7 \mathrm{~ms}\left(t_{2},{ }^{15} \mathrm{~N}\right)$, and $50 \mathrm{~ms}\left(\mathrm{t}_{3},{ }^{1} \mathrm{H}\right)$, using 4 scans per fid and a total measuring time of 19 hours for all three interleaved spectra. In case of the $\mathrm{CBCA}(\mathrm{CO}) \mathrm{NH}$, each interleaved spectrum was recorded as a $46^{*} \times 25^{*} \times 512^{*}$ data matrix with acquisition times of $4.9 \mathrm{~ms}\left(t_{1},{ }^{13} \mathrm{C}^{\alpha / \beta}\right), 17.5 \mathrm{~ms}\left(t_{2},{ }^{15} \mathrm{~N}\right)$, and $50 \mathrm{~ms}\left(\mathrm{t}_{3},{ }^{1} \mathrm{H}\right)$ using 8 scans per fid and a total measuring time of 48 hours for all four interleaved spectra. Data were processed and analyzed using NMR-PIPE/NMRDRAW [48]. 


\subsection{Results and discussion}

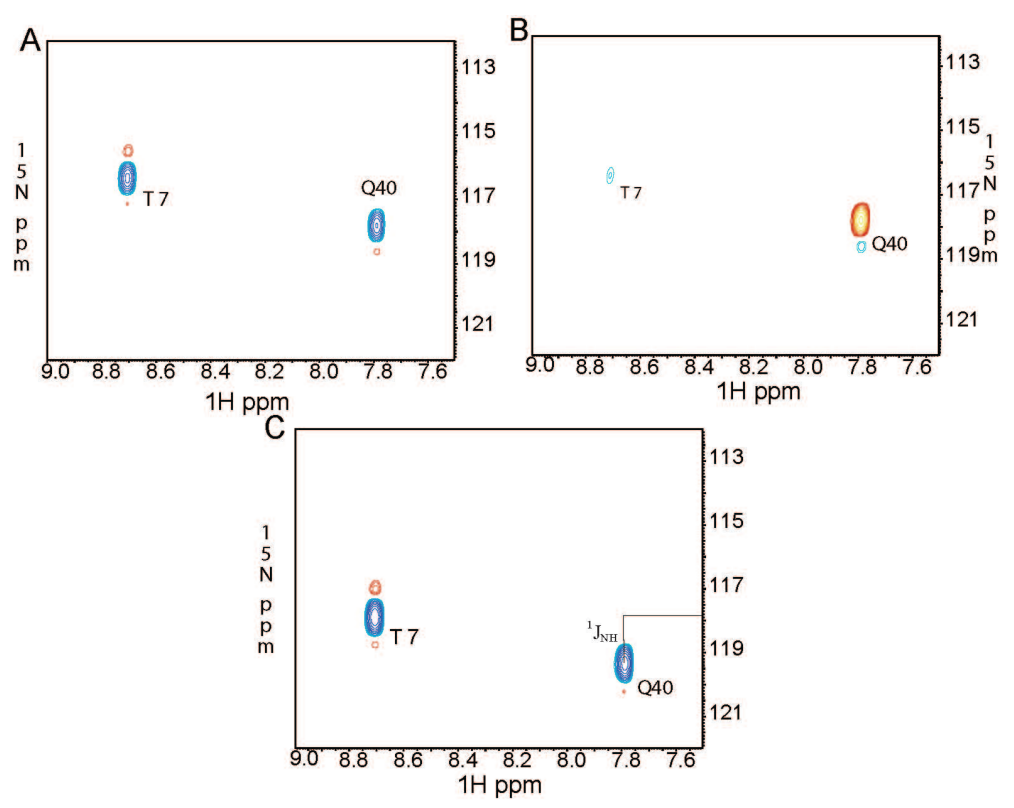

Figure 3.3: Selected regions of the modified TROSY-HNCO spectrum of ubiquitin in isotropic solution. A) reference spectrum, B) ${ }^{1} J_{C^{\prime} N}$-attenuated spectrum and C) ${ }^{1} J_{N H}$-shifted spectrum. Dashed contours denote negative intensities. Selected regions have a C' frequency of $171.1 \mathrm{ppm}$.

Selected regions of the spectra measured with these pulse sequences for ubiquitin in isotropic solution are shown in Figures. 3.3 and 3.4. In Figure 3.3A the first interleaved TROSY-HNCO spectrum is shown, which is used as reference for extraction of both ${ }^{1} \mathrm{~J}_{C^{\prime} N}$ and ${ }^{1} \mathrm{~J}_{N H}$ couplings. The second interleaved spectrum is shown in Figure $3.3 \mathrm{~B}$, in which signal intensities are modulated according to the ${ }^{1} \mathrm{~J}_{C^{\prime} N}$ coupling. In Figure $3.3 \mathrm{C}$ resonances are not attenuated compared to Figure $3.3 \mathrm{~A}$, but shifted in the ${ }^{15} \mathrm{~N}$ dimension by ${ }^{1} \mathrm{~J}_{N H} \mathrm{~Hz}$ from the reference spectrum of Figure 3.3A. Figure 3.4 shows selected regions of the interleaved $\mathrm{CBCA}(\mathrm{CO}) \mathrm{NH}$ experiments. In Figure $3.4 \mathrm{~A}$ the reference spectrum is shown and in Figure $3.4 \mathrm{~B}$ and $\mathrm{C}$ signal intensities are modulated by the ${ }^{1} \mathrm{~J}_{C \alpha H \alpha}$ and ${ }^{1} \mathrm{~J}_{C \beta H \beta}$ couplings. Figure 3.4 $\mathrm{D}$ encodes the ${ }^{1} \mathrm{~J}_{C \alpha C^{\prime}}$ coupling.

\subsubsection{Sensitivity consideration for $\mathrm{CBCA}(\mathrm{CO}) \mathrm{NH}$ experiment}



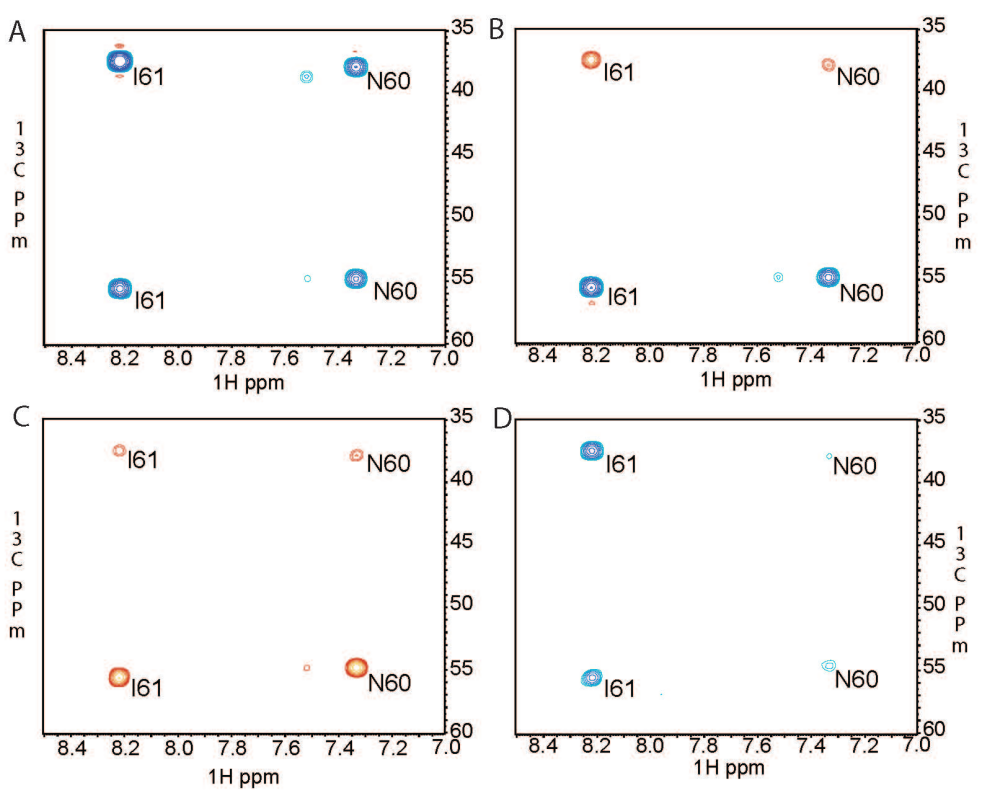

Figure 3.4: Selected regions of the modified $\mathrm{CBCA}(\mathrm{CO}) \mathrm{NH}$ spectrum of ubiquitin in isotropic solution. $A$ is the reference spectrum. $B$ and $C$ are the attenuated spectra for measuring $\mathrm{C}_{\alpha}-\mathrm{H}_{\alpha}$ and $\mathrm{C}_{\alpha}-\mathrm{H}_{\beta}$ couplings. D) shows the spectrum for measuring $\mathrm{C}_{\alpha}-\mathrm{C}^{\prime}$ couplings. Selected regions have a ${ }^{15} \mathrm{~N}$ frequency of $116.2 \mathrm{ppm}$.

A $\mathrm{CBCA}(\mathrm{CO}) \mathrm{NH}$ experiment is much less sensitive than a conventional $\mathrm{HNCO}$ due to the larger number of transfer steps and the fast relaxation of transverse $\mathrm{C}_{\alpha}$ and $\mathrm{C}_{\beta}$ magnetization. For a $11 \mathrm{kDa}$ protein such as ribonuclease $\mathrm{T} 1$ the $\mathrm{CBCA}(\mathrm{CO}) \mathrm{NH}$ is a factor of three less sensitive than a HNCO experiment (Bruker Avance 3D/Triple Resonance User Manual). For larger proteins the relative sensitivity of the $\mathrm{CBCA}(\mathrm{CO}) \mathrm{NH}$ is even further reduced. However, following considerations have to be taken into account for measurement of ${ }^{1} \mathrm{D}_{C \alpha C^{\prime}}$ couplings. The sensitivity of the $\operatorname{HNCO}\left(\alpha / \beta-\mathrm{C}^{\prime} \mathrm{C}_{\alpha^{-}} J\right)$ experiment proposed by Permi et al.[15], for example, is reduced by a factor of 0.9 due to the insertion of a filter element with a duration of $9 \mathrm{~ms}$ and due to an extended ${ }^{13} \mathrm{C}$ evolution time of about $30 \mathrm{~ms}$, which is required to obtain sufficient resolution for accurate measurement of splittings (assuming a C' transverse relaxation time of $117 \mathrm{~ms}$ ). Moreover, the intensity of a single resonance is split into two reducing the sensitivity by $45 \%$ overall. For the approach proposed here, on the other 
hand, only a single additional, conventional $\mathrm{CBCA}(\mathrm{CO}) \mathrm{NH}$ experiment is required as the reference experiment is already available either from the measurement of ${ }^{1} \mathrm{D}_{C \alpha H \alpha} /{ }^{1} \mathrm{D}_{C \beta H \beta}$ couplings or from conventional backbone assignment. No additional delays or pulses had to be introduced into the $\mathrm{CBCA}(\mathrm{CO}) \mathrm{NH}$ pulse sequence avoiding increased relaxation losses or a higher sensitivity towards pulse imperfections. The accuracy of the ${ }^{1} \mathrm{~J}_{C \alpha C^{\prime}}$ couplings obtained from the $\mathrm{CBCA}(\mathrm{CO}) \mathrm{NH}$ is further improved, as splittings can be obtained from both the $\mathrm{C}_{\alpha}$ and the $\mathrm{C}_{\beta}$ resonance. The corresponding values can be averaged, such that the sensitivity of the $\mathrm{CBCA}(\mathrm{CO}) \mathrm{NH}$ (in terms of measurement of couplings) is effectively increased by a factor of $\sqrt{2}$. Therefore, for small to medium sized proteins such as ribonuclease T1, measurement of ${ }^{1} \mathrm{D}_{C \alpha C^{\prime}}$ couplings simultaneously with ${ }^{1} \mathrm{D}_{C \alpha H \alpha} /{ }^{1} \mathrm{D}_{C \beta H \beta}$ values has a similar efficiency as separate measurement of ${ }^{1} \mathrm{D}_{C \alpha C^{\prime}}$ couplings with a $\operatorname{HNCO}\left(\alpha / \beta-\mathrm{C}^{\prime} \mathrm{C}_{\alpha}-J\right)$ experiment.

Measurement of ${ }^{1} \mathrm{~J}_{C \alpha C^{\prime}}$ couplings as proposed herein has the following favorable properties: (i) Opposite to $\alpha / \beta$-filtered spin-state selective experiments $J$-mismatch is not a problem in quantitative $J$-correlation experiments. This is particularly important for measurement of RDCs where the filter period no longer matches the $J+D$ value. (ii) Despite the lower resolution of a $\mathrm{CBCA}(\mathrm{CO}) \mathrm{NH}$ compared a $\mathrm{HNCO}$ experiment, a very complete set of ${ }^{1} \mathrm{~J}_{C \alpha C^{\prime}}$ couplings can be obtained as in case of $\mathrm{C}_{\alpha}$ overlap the coupling might be extracted from the $\mathrm{C}_{\beta}$ resonance. (iii) For extraction of ${ }^{1} \mathrm{~J}_{C \alpha C^{\prime}}$ couplings no additional experiment has to be processed, peak picked and analysed. ${ }^{1} \mathrm{~J}_{C \alpha C^{\prime}}$ couplings are obtained in parallel with the ${ }^{1} \mathrm{~J}_{C \alpha H \alpha}$ and ${ }^{1} \mathrm{~J}_{C \beta H \beta}$ couplings, minimizing the number of errors in extracted dipolar coupling values. (iv) The proposed pulse sequences allow simultaneous measurement of RDCs such that they will be in agreement with a unique effective alignment tensor even if the protein degrades/aggregates rapidly. With the $\mathrm{CBCA}(\mathrm{CO}) \mathrm{NH}$ this is possible for ${ }^{1} \mathrm{~J}_{C \alpha C^{\prime}},{ }^{1} \mathrm{~J}_{C \alpha H \alpha}$, and ${ }^{1} \mathrm{~J}_{C \beta H \beta}$ couplings without increased resonance overlap and employing a common reference experiment.

\subsubsection{Sensitivity consideration for TROSY-HNCO experiment}

In case of the 3D TROSY-HNCO, the incremented delay that was introduced for the extraction of the ${ }^{1} \mathrm{~J}_{N H}$ couplings (Figure 3.1; a, (ii)) slightly lowers the sensitivity of the third 
interleaved TROSY-HNCO spectrum compared to the reference experiment. In addition, during this additional delay transverse relaxation proceeds with an effective rate that is given by the average of the individual ${ }^{15} \mathrm{~N}$ multiplet components. Therefore, for large molecules a compromise between separation of the upfield and downfield component (which increases with $\kappa$ ) and the sensitivity of this experiment has to be made. For large molecules it might be advantageous to reduce $\kappa$. At the same time, however, the $180^{\circ}$ pulses on ${ }^{15} \mathrm{~N}$ and ${ }^{13} \mathrm{C}$ ' could be applied simultaneously after a time $T=16 \mathrm{~ms}$, such that $2 \mathrm{~T}$ would be reduced to $32 \mathrm{~ms}$ as in a conventional TROSY-HNCO.

${ }^{1} \mathrm{~J}_{C^{\prime} N}$ couplings can be measured separately using a non-decoupled TROSY-HNCO, in which ${ }^{1} \mathrm{~J}_{C^{\prime} N}$ values are obtained from antiphase splittings in the ${ }^{15} \mathrm{~N}$ dimension [32]. In order to resolve the small ${ }^{1} \mathrm{~J}_{C^{\prime} N}$ couplings a rather long ${ }^{15} \mathrm{~N}$ acquisition time is required, which is best implemented in a semi-constant time fashion and set to a maximum value, $t_{2}^{\max }$, roughly equal to the transverse ${ }^{15} \mathrm{~N}$ relaxation time $t_{2}$ [90]. For example, for deuterated EIN (transverse relaxation time of the downfield component at 600 and $800 \mathrm{MHz}: 118$ and $131 \mathrm{~ms}$, respectively) a ${ }^{15} \mathrm{~N}$ acquisition time of $115 \mathrm{~ms}$ was employed. Comparison of the relaxation losses in the quantitative $J$-correlation experiment, in which the ${ }^{15} \mathrm{~N}$ magnetization is 33 ms longer in the transverse plane than in a regular TROSY-HNCO, with those in a nondecoupled TROSY-HNCO, with a semi-constant time evolution $t_{2}^{\max }=T_{2}\left({ }^{15} \mathrm{~N}\right)$, indicates that the quantitative $J$-correlation experiment is more sensitive for $T_{2}\left({ }^{15} \mathrm{~N}\right)>100 \mathrm{~ms}[20]$. $T_{2}\left({ }^{15} \mathrm{~N}\right)$ values larger than $100 \mathrm{~ms}$ are commonly observed at room temperature for globular proteins with a molecular weight $<16 \mathrm{kDa}$, for the downfield component of deuterated proteins up to $30 \mathrm{kDa}$, such as EIN, or for large protonated, but natively unfolded proteins. For example, for ubiquitin $\left(T_{2}\left({ }^{15} \mathrm{~N}, 25^{\circ} \mathrm{C}\right) \sim 160 \mathrm{~ms} ; T_{2}\left({ }^{15} \mathrm{~N}, 7^{\circ} \mathrm{C}\right) \sim 105 \mathrm{~ms}\right)$ the quantitative $J$ correlation experiment is $15 \%$ and $2 \%$ more sensitive than the non-decoupled experiment at $25^{\circ} \mathrm{C}$ and $7^{\circ} \mathrm{C}$, respectively. On the other hand, for a relaxation time of $74 \mathrm{~ms}\left(\sim t_{2}\left({ }^{15} \mathrm{~N}\right)\right.$ of protonated EIN) the non-decoupled HNCO would be more sensitive by $20 \%$. Therefore, for transverse relaxation times larger than $100 \mathrm{~ms}$ simultaneous measurement of ${ }^{1} \mathrm{~J}_{N H}$ and ${ }^{1} \mathrm{~J}_{C^{\prime} N}$ couplings as proposed here achieves a time saving that corresponds to about one conventional TROSY-HNCO. Note, that this does not even take into account that the reference 
experiment (Figure 3.1; a, (i)) or the shifted experiment (Figure 3.1; a, (ii)) can be used for backbone assignment, which is difficult with the non-decoupled TROSY-HNCO. A further drawback of the non-decoupled TROSY-HNCO is that the overlap is more pronounced.

\subsubsection{Error estimation}

The random error in coupling values obtained by quantitative $J$-correlation methods mainly depends on the intensity of the reference spectrum (abbreviated as) $\mathrm{I}_{\text {ref }}$, and the random noise (denoted as) $\sigma$ in the attenuated spectra. This random error in coupling values got by quantitative $J$-correlation method is approximated as, $\sigma /\left[2 \pi(\zeta+\Delta) I_{\text {ref }}\right]$ where $2(\zeta+\Delta)$ is the total duration in which that particular coupling is active in the attenuated spectrum. So for a signal to noise ratio of 50 in the reference spectrum of the $\mathrm{CBCA}(\mathrm{CO}) \mathrm{NH}$ experiment, the random error in ${ }^{1} \mathrm{~J}_{C \alpha C^{\prime}}[2(\zeta+\Delta)=18 \mathrm{~ms}]$ will be $0.35 \mathrm{~Hz}(\Delta=4.85 \mathrm{mS}$, $\zeta=4.5 \mathrm{mS}$ ). Except for couplings between ${ }^{13} \mathrm{C}^{\prime}$ and carbons resonating in the $\mathrm{C}_{\alpha}$ region, all other couplings attenuate the reference and attenuated spectrum in the same way, and therefore do not affect the derived coupling. The inter-residue ${ }^{2} \mathrm{~J}_{C \alpha C^{\prime}}$ coupling, on the other hand, affect the attenuated and the reference spectrum differentially. The magnitude of ${ }^{2} \mathrm{~J}_{C \alpha C^{\prime}}$ with the next residue being in cis position is $\leq 3 \mathrm{~Hz}$ [91. This attenuates the reference spectrum by less than $0.4 \%$ and the attenuated spectra by $1.5 \%$, and the $I_{\text {att }} / I_{\text {ref }}$ ratio is decreased by $1.2 \%$, resulting in a coupling which is at most $1.2 \%$ closer to $55 \mathrm{~Hz}$ than its real value. When the bonds are trans, as it might be the case for residues preceding proline, the coupling could be as large as $6 \mathrm{~Hz}$ [91] resulting in a more pronounced overestimation of the derived coupling. However, these residues are any way not detected in $\mathrm{CBCA}(\mathrm{CO}) \mathrm{NH}$ experiments. Due to the fact that the selectivity of the $\mathrm{C}_{\alpha}$ inversion pulse is not perfect, other long-range couplings that may affect the derived ${ }^{1} \mathrm{~J}_{C \alpha C^{\prime}}$ value can include ${ }^{2} \mathrm{~J}_{C^{\prime} C \beta}$ and ${ }^{3} \mathrm{~J}_{C^{\prime} C \gamma}$. These couplings, however, are comparatively smaller. Assuming ${ }^{2} \mathrm{~J}_{C^{\prime} C \beta}$ and ${ }^{3} \mathrm{~J}_{C^{\prime} C \gamma}$ values of $3 \mathrm{~Hz}$ and $4 \mathrm{~Hz}$, respectively, the cumulative effect of all the three couplings would be to make the coupling closer to $55 \mathrm{~Hz}$ by less than $4.6 \%$. So a coupling of $52.0 \mathrm{~Hz}$ will appear as $52.1 \mathrm{~Hz}$ and for an actual coupling of $54 \mathrm{~Hz}$ the derived coupling would be 54.05 $\mathrm{Hz}$, which typically is considerably smaller than the random error. 

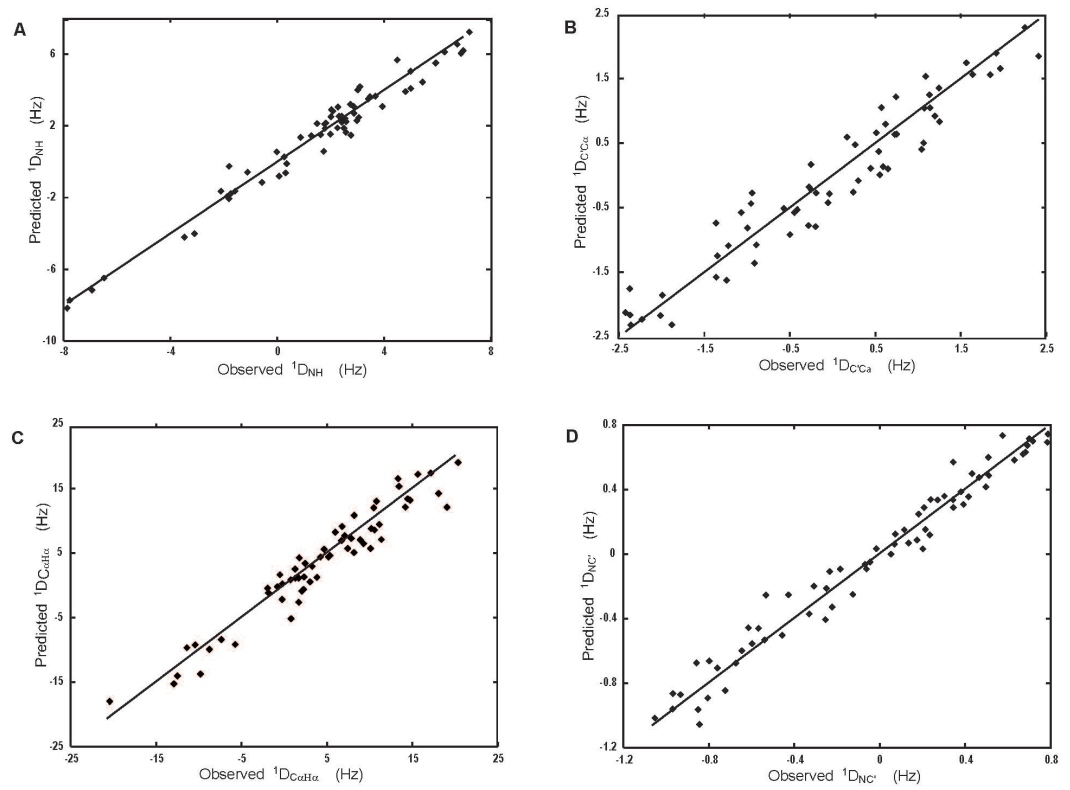

Figure 3.5: Correlation between observed dipolar couplings and values back-calculated from the crystal structure of ubiquitin (PDB code: 1ubq). The correlation coefficients are 0.98, 0.96, 0.98 and 0.96 for ${ }^{1} \mathrm{D}_{N H},{ }^{1} \mathrm{D}_{C \alpha H \alpha},{ }^{1} \mathrm{D}_{C^{\prime} N}$ and ${ }^{1} \mathrm{D}_{C \alpha C^{\prime}}$ couplings, respectively
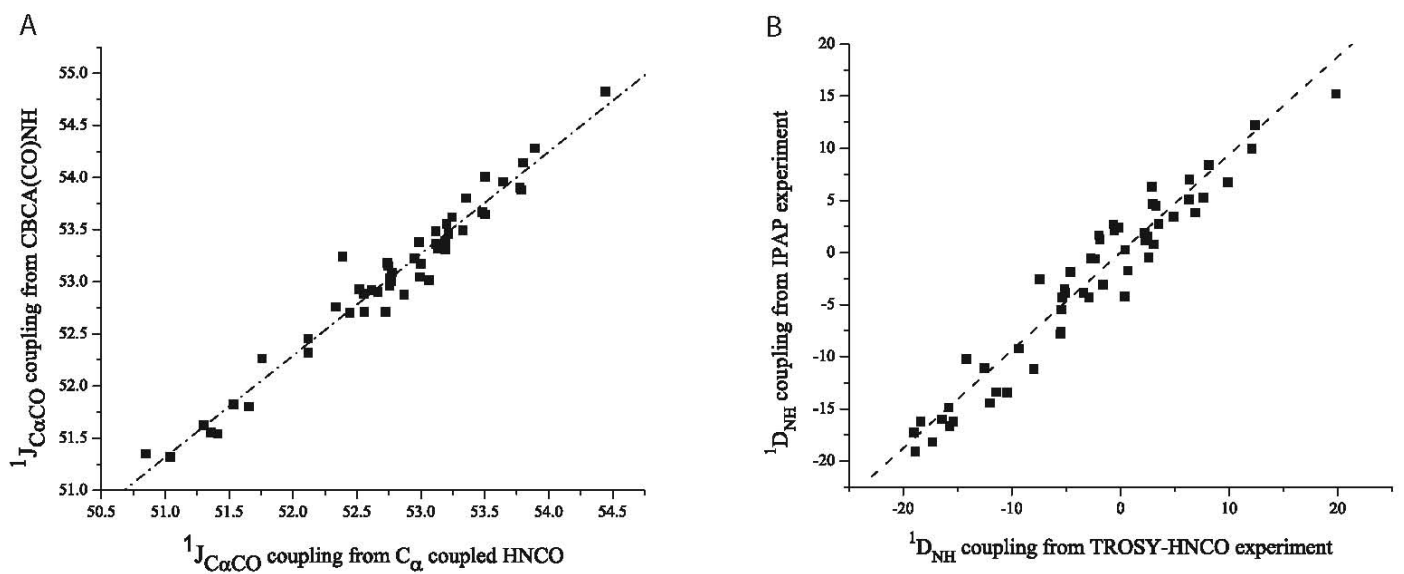

Figure 3.6: A Correlation between ${ }^{1} \mathrm{~J}_{C^{\alpha} C^{\prime}}$ measured using the $\mathrm{CBCA}(\mathrm{CO}) \mathrm{NH}$ experiment and the same coupling measured using the $\mathrm{C}_{\alpha}$ coupled $\mathrm{HNCO}$ experiment for protein G. B Correlation between measured ${ }^{1} \mathrm{D}_{N H}$ dipolar couplings using TROSY-HNCO and IPAP HSQC for DcuS-PD. The correlation coefficients were 0.98 and 0.97 respectively. 
Hence, extracted coupling values do not contain any large systematic errors. This is also evident from isotropic ${ }^{1} \mathrm{~J}_{C \alpha C^{\prime}}$ couplings measured with the pulse sequence presented here, when compared to those obtained from a $\mathrm{C}_{\alpha}$-coupled $\mathrm{HNCO}$ experiment recorded on the third immunoglobulin-binding domain of streptococcal protein $\mathrm{G}$ (56 residues). The coupling values from these two measurements have a correlation of 0.98 (Figure 3.6 A). Comparison of ${ }^{1} \mathrm{D}_{N H}$ coupling measured for DcuS-PD with the 3D TROSY-HNCO experiment presented here and those obtained from a 2D IPAP-HSQC have a correlation of 0.97 (Figure 3.6B)

\subsubsection{Correlation of measured RDCs with structure}

Figure 3.5 compares experimental dipolar couplings with those back-calculated from the $1.8 \AA$ X-ray structure of ubiquitin [92] using singular-value decomposition as implemented in the program PALES [49]. Correlation factors of 0.98, 0.96, 0.98 and 0.96 are obtained for ${ }^{1} \mathrm{D}_{N H},{ }^{1} \mathrm{D}_{C \alpha H \alpha},{ }^{1} \mathrm{D}_{C^{\prime} N}$ and ${ }^{1} \mathrm{D}_{C \alpha C^{\prime}}$ couplings, respectively. This demonstrates that the pulse sequences presented here allow accurate measurement of $J$ and dipolar couplings in medium sized proteins. Residual dipolar couplings measured in DcuS (17 kDa) 4 were used for the structure determination of this protein [44].

\subsection{Conclusions}

We have presented a simple strategy for simultaneous measurement of scalar and dipolar couplings in ${ }^{13} \mathrm{C},{ }^{15} \mathrm{~N}$-labeled proteins. More than one type of coupling is extracted from a single reference experiment, thereby reducing the required measurement time for small to medium sized globular, as well as larger, unfolded proteins. The couplings are obtained either using the quantitative $J$-correlation approach or accordion spectroscopy; a second spectrum is recorded in which the signal intensities are modulated or signals are shifted according to the coupling of interest. Hence, simultaneous measurement of dipolar couplings does not result in an increase in resonance overlap compared to when the couplings are measured sequentially. The strategy presented is general and can be applied to various 3D experiments.

The interleaved 3D TROSY-HNCO and CBCA $(\mathrm{CO}) \mathrm{NH}$ experiments presented here allow measurement of four backbone RDCs and ${ }^{1} \mathrm{D}_{C \beta H \beta} \cdot{ }^{1} \mathrm{D}_{N H},{ }^{1} \mathrm{D}_{C \alpha H \alpha},{ }^{1} \mathrm{D}_{C^{\prime} N}$ and ${ }^{1} \mathrm{D}_{C \alpha C^{\prime}}$ can be 
used in a conventional structure calculation, enabling accurate determination of peptide plane orientations [72], or protein fold determination even in the absence of NOEs [46, 73, 74]. The combination of ${ }^{1} \mathrm{D}_{C \alpha H \alpha},{ }^{1} \mathrm{D}_{C \beta H \beta}$, and ${ }^{1} \mathrm{D}_{C \alpha C^{\prime}}$ provides $\chi 1$ rotamer information, even in the absence of a backbone structure [84]. Moreover, the reference spectra, which are used here for extraction of couplings, can be used for traditional backbone assignment, further reducing the total required measurement time in a NMR structure determination project. In the broader sense of speeding up structure determination by NMR (e.g. as required for Structural Genomics applications), our strategy also has the advantage that peak picking, refinement of picked peaks and reassignment of resonances only has to be done for the reference spectrum, as long as the quantitative $J$-correlation approach is employed. For extraction of the ${ }^{1} \mathrm{D}_{C \alpha C^{\prime}}$ coupling no additional experiment has to be processed, peak picked and analyzed. ${ }^{1} \mathrm{D}_{C \alpha C^{\prime}}$ couplings are obtained in parallel with the ${ }^{1} \mathrm{D}_{C \alpha H \alpha}$ and ${ }^{1} \mathrm{D}_{C \beta H \beta}$ couplings. In addition, if the anisotropic medium is unstable or the protein degrades rapidly, as it is the case for DcuS, measured residual dipolar couplings will be in agreement with a unique effective alignment tensor. 


\section{Chapter 4}

\section{NMR structural studies on the periplasmic domain of DcuS and CitA}

\subsection{Introduction}

Cells respond to changes in their physical and chemical environment by generating intracellular signals that lead to the modification of their structure, metabolism and movement. Most of these signals originate in one side of the membrane and require the sensing, and then the transfer of the signal to the other side of the membrane facilitating the regulatory responses [93]. Signal transduction across biological membrane is very important for the ability of cells to integrate and process environmental information. In most prokaryotes and a few eukaryotes this is usually performed by an evolutionarily conserved class of proteins, called the two component system [94, 95, 96]

The two-component system in their simplest form consist of two modular proteins, a sensor kinase and a response regulator [97, 98, 99]. In prokaryotes, the sensor protein mostly belongs to a larger family of protein kinases called Histidine kinases, which has the conserved histidine residue in the cytoplasmic domain that upon stimulus functions as a site for autophosphorylation. Autophosphorylation is carried out with the help of other subunit of sensor kinase within a homodimer [100, 101, 102, 103]. The response regulator catalyzes phosphoryl transfer from the phospho-histidine of the Histidine kinase to a conserved aspartate in its own regulatory domain, and controls the expression of target genes by transcriptional activation or repression [104]. The majority of response regulators are transcription factors with DNA-binding effector domains [99. Refer Figure 4.1 for the schematic representation of the working of a typical two component system. 


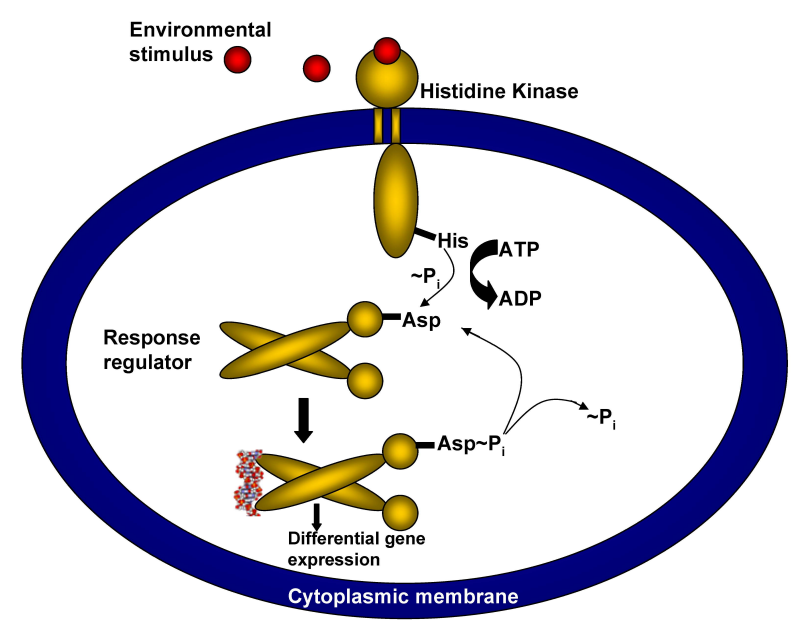

Figure 4.1: Schematic representation of a two component system: Transmembrane sensor histidine kinase act as the sensor domain, which upon stimulus undergoes autophosphorylation at the conserved histidine in the kinase core with an ATP getting converted to an ADP. The phosphoryl group is then transferred to the response regulator which in turn binds to the DNA starting the transcription of genes.

\subsubsection{Histidine Kinase (HK)}

In a typical two component system, a sensor histidine kinase monitor external stimuli and transmit this information to the response regulator by a phosphorylation event.

The main structural feature of the Histidne Kinase family include the diverse sensing domain and the characteristic kinase core composed of dimerization domain and an ATP/ADP-binding phosphotransfer or catalytic domain [99, 105]. In trans membrane histidine kinase (see Figure 4.2 the sensing domain is connected to the kinase core by a linker domain.

\section{Kinase Core}

All protein in the HK family have a characteristic kinase core composed of a dimerization domain and an ATP/ADP binding phospho-transfer or catalytic domain. [106] X-ray structure of C terminal half of T.maritima CheA protein [107] and the NMR solution structure of 


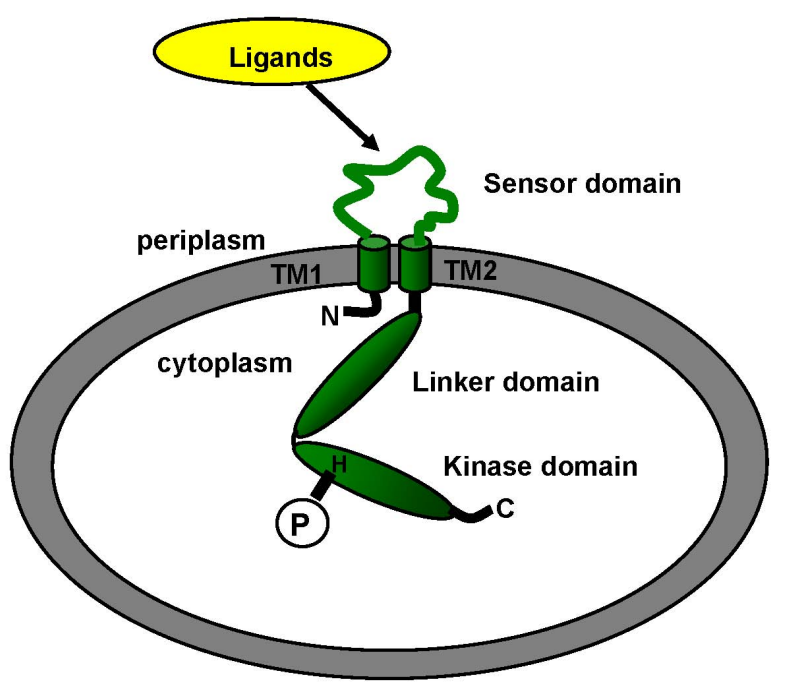

Figure 4.2: Schematic representation of sensory trans-membrane histidine kinase with the sensor domain present outside the cytoplasm. Ligands are sensed in the the periplasm with the help of the sensory domain. The linker domain connects the periplasmic domain to the kinase core which has the conserved histidine where upon binding of ligand, autophosphorylation take place.

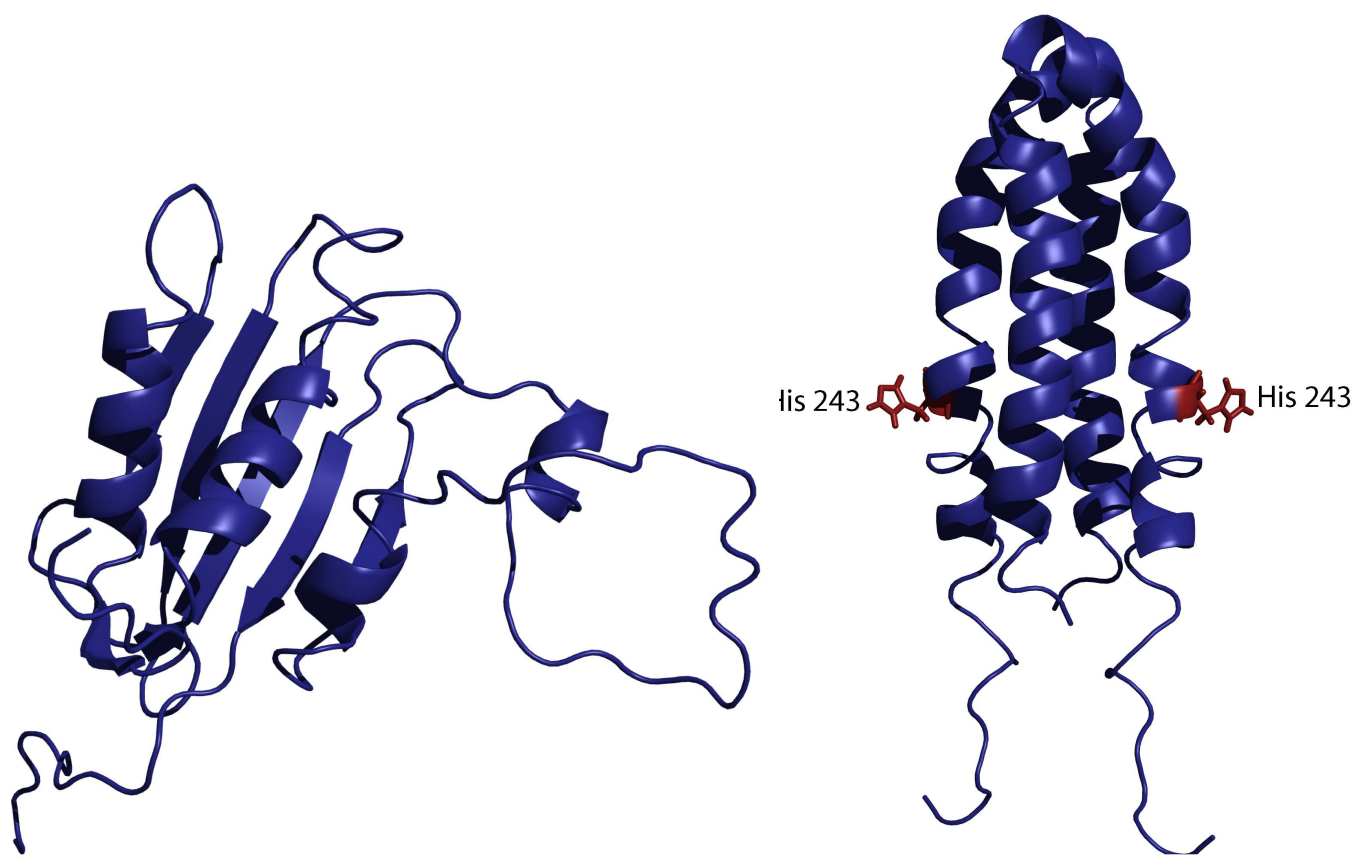

Figure 4.3: The kinase core of histidine protein kinases. The NMR structure of the (left) catalytic and (right) dimerization domains of E. coli EnvZ. The dimerization domain of EnvZ (right), like that of most histidine protein kinases, contains a conserved Histdine (blue) that is the site of phosphorylation. 
catalytic domain [108], of E.coli EnvZ revealed an $\alpha / \beta$ sandwich fold consisting of five anti parallel $\beta$ strands and $3 \alpha$ helices (Figure 4.3 left). The dimerization domains of both EnvZ [109] and CheA form anti parallel four-helix bundles. The dimerization domain of EnvZ, like those of the majority of HKs, houses the conserved histidine, which is positioned midway along the exposed face of first helix. (Figure 4.3 right).

\section{Linker domain}

Its the least understood domain of HKs. But studies indicate that these linker regions are critical for proper signal transduction [110, 111, 112]. This domain may promote intra molecular association or as suggested by mutational analysis of the EnvZ linker region, correct structural alignment of monomers within the HK dimer [113].

\section{Sensing domain}

Signal transduction cascades begin with signal detection by the sensor domain. These domain senses wide range of ligands, redox potential and ions. These diverse sensing domains share little primary sequence similarity, indicating that they are designed for specific ligand/stimulus interactions. One of the best known sensing module incorporated into the sensing domain is the versatile PAS domains [114, 115].

PAS (PER-ARNT-SIM) domains are a family of sensor protein domains involved in signal transduction in a wide range of organism [116, 117]. The PAS module was first identified in the Drosophila clock protein PER and the basic helix-loop-helix containing transcription factors ARNT (aryl-hydrocarbon receptor nuclear translocator) in mammals and SIM (singleminded proteins) in insects [118]. A typical representative of the PAS domain is photoactive yellow protein(PYP) [119], a phtoreceptor to be involved in a photoactive response of the bacterium Ectothiorhodospira halophila to intense blue light [120].

Its structure has an $\alpha / \beta$ fold characterized by a central, twisted, 6 stranded anti parallel $\beta$ sheet flanked on both sides by loops and helices. Helices $\alpha 3$ and $\alpha 4$, the short strand $\beta 3$, the loop connecting the $\beta 3$ strand to the helix $\alpha 5$, the helix $\alpha 5$, span across the other side of $\beta$ sheet forms the binding pocket for the light sensitive chromophore p-coumeric 


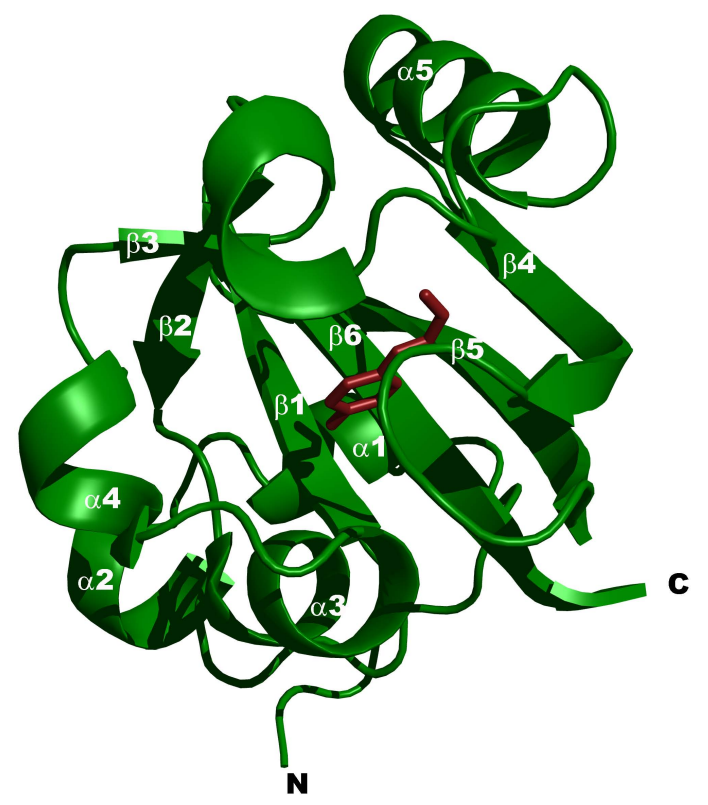

Figure 4.4: X-ray Structure of photo active yellow protein(PYP) revealing a PAS fold . The ligand, 4-hydroxycinnamyl chromophore position shown in sticks (red).

acid (see Figure 4.4). Although the PAS domains have little amino acid similarity, their three-dimensional structures appear to be conserved [121].

In the past two decades, hundreds of two component systems have been identified. However, many aspects of their molecular function remain obscure. In particular, for the ligand-binding sensor kinases little is known about such fundamental processes as the stereo chemistry of ligand-binding, the nature of the associated conformational changes, and the mechanism of signal transduction to the cytoplasmic kinase domain.

The aim behind the study was to understand the signal perception and transduction of the signal from the sensor domain to the cytosolic domain. As a first step we describe the NMR studies on the soluble periplasmic domain of CitA and DcuS with and without their ligand. Here we describe the NMR solution structure of the ligand free DcuS sensory periplasmic domain and the X-ray structure of ligand bound and free structure of sensory domain of 
CitA. These structures reveal a PAS fold, and are the first of the few determined structures having this fold, present outside the cytoplasm [116]. 


\subsection{Periplasmic domain of the sensory domain of the two component fumarate sensor DcuS}

The fumarate sensor DcuS is a prototype for a two component sensory histidine kinase with signal perception in the periplasm, trans membrane signal transfer [122, 123], and autophosphorylation of a histidine residue in the kinase domain in the cytoplasm [124]. DcuS belongs to the CitA family of sensors that are specific for sensing di- and tricarboxylates [122, 123, 125, 126]. The periplasmic domain of the histidine autokinase CitA works as a highly specific citrate receptor, whereas DcuS uses any type of C4-dicarboxylate, like fumarate, succinate, and malate, as a stimulus [122, 125, 126, 127]. DcuS is predicted to consist of two transmembrane helices and of a periplasmic sensory domain enclosed by the trans membrane helices. The second trans membrane helix is followed by a cytoplasmic PAS domain of unknown function and the kinase with the consensus histidine residue for autophosphorylation. Previous studies suggest that fumarate sensing occurs in the periplasmic domain [122, 125, 127]. After autophosphorylation by DcuS the response regulator DcuR of the DcuSR system (Figure 4.5) activates the expression of target genes like $\mathrm{dcuB}$ and frdABCD encoding an anaerobic fumarate carrier DcuB and fumarate reductase [125, 126].

Biochemical studies on DcuS reconstituted in liposomes indicated that in the presence of fumarate and other dicarboxylates, kinase activity of DcuS is stimulated [122]. But the binding site, or the exact nature of the stimulus were yet to be fully characterized. Moreover, there was a lack of three dimensional structural information for any domain of DcuS or that of its homologues. This study was initiated to address these issues. Hence as a first step to understand the signal perception, NMR structural studies on the periplasmic domain of DcuS were performed. The structure of DcuS-PD derived using NOE was refined using RDCs for better convergence of the structure. The structure of DcuS-PD could be solved only without bound C4-dicarboxylates which precludes direct identification of the effector binding site. Titration experiments were necessary to establish the sensing mode of $\mathrm{C} 4$ dicarboxylates by DcuS-PD. 


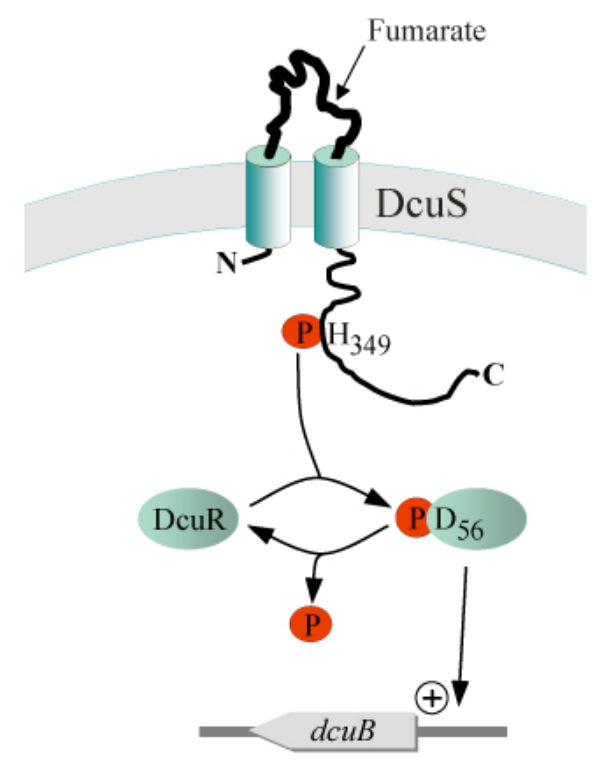

Figure 4.5: Schematic representation of DcuS-DcuR system. After the autophosphorylation of DcuS, phosphoryl group on histidine 349 in DcuS is transferred to aspartate 56 in DcuR. DcuR then binds to the dcuB codon, triggering the expression of the genes for fumarate uptake and respiration.

\subsubsection{Material and Methods}

The periplasmic domain of DcuS was produced in the lab of Prof. Gotfried Unden in University of Mainz. DcuS-PD was overproduced in E-coli strain carrying the $\mathrm{DcuS}_{45-180}$ expression plasmid [44]. The assignments were taken from the previously published assignment of histidine tagged DcuS-PD [128]. The assignment of DcuS-PD without the His-tag differs only slightly from the one with the His-tag. NMR measurements were carried out with the ${ }^{15} \mathrm{~N}$ ${ }^{13} \mathrm{C}$ labeled protein received from Prof. Unden's group in Mainz. The wild type protein containing samples were usually around $1 \mathrm{mM}$, in a $50 \mathrm{mM}$ sodium phosphate buffer at $\mathrm{pH}$ 7.0, including $200 \mathrm{mM} \mathrm{NaCl}, 0.8 \mathrm{mM}$ CHAPS, $50 \mathrm{mM}$ Glycine, $50 \mathrm{pM}$ of PefablocSC, $0.01 \%$ $\mathrm{NaN}_{3}$ and $\mathrm{H}_{2} \mathrm{O} / \mathrm{D}_{2} \mathrm{O} .90 / 10 \%$. NMR samples were stable for a week after which it degraded gradually. 
NOESY experiments were measured and analysed by Dr. Lucia Pappalardo and Dr. Wolfgang Peti. Structural calculations were done by Dr. Jochen Junker in our lab.

Hetronuclear NOE experiment was measured with 5 second recycling delay

\section{RDC measurements}

To refine the structure, several sets of RDCs were measured. Protein was oriented in bacterial filamentous phages. The concentration of the phage in the protein sample was 10 $\mathrm{mg} / \mathrm{ml}$ giving deuterium quadrapolar splitting of $9 \mathrm{~Hz}$. A set of ${ }^{1} \mathrm{D}_{N H},{ }^{1} \mathrm{D}_{N C^{\prime}},{ }^{1} \mathrm{D}_{C C \alpha}$ and ${ }^{1} \mathrm{D}_{\mathrm{C} \alpha \mathrm{H} \alpha}$ RDCs of DcuS-PD were measured using the pulse sequence described in chapter 3 . The magnitude of the alignment tensor (Da) obtained from histogram of measured dipolar couplings is $10.7 \mathrm{~Hz}$ for ${ }^{1} \mathrm{D}_{N H}$ and the corresponding rhombicity (R) is 0.63 . RDCs were applied in the structural calculation with different weight factors $1.0\left({ }^{1} \mathrm{D}_{N H}\right), 0.4\left({ }^{1} \mathrm{D}_{C \alpha H \alpha}\right)$, $4.4\left({ }^{1} \mathrm{D}_{N C^{\prime}}\right)$ and $2.5\left({ }^{1} \mathrm{D}_{C C \alpha}\right)$. The weighting factor used here reflect the gyromagnetic ratios of the nuclei involved in the coupling and also the bond distances.

\section{Effector titration of DcuS-PD}

To study the effector binding, wild type DcuS-PD and the mutant forms, DcuS(F120M)PD, DcuS(R147A)-PD, and DcuS(H110A)-PD were dissolved in a NMR buffer at pH 7.0 containing $45 \mathrm{mM}$ sodium/potassium phosphate, $10 \% \mathrm{D}_{2} \mathrm{O}, 200 \mathrm{mM} \mathrm{NaCl}, 0.01 \% \mathrm{NaN}_{3}$, $50 \mathrm{uM}$ Pefabloc, $50 \mathrm{mM}$ glycine, and $4.5 \mathrm{mM}$ imidazole. Titration with the effectors were conducted by stepwise addition of properly buffered fumarate and tartrate solutions ( $\mathrm{pH} 7.0$ and upto $300 \mathrm{mM}$ final concentration) to a sample of $1.2 \mathrm{mM}$ of DcuS-PD. ${ }^{15} \mathrm{~N}-{ }^{1} \mathrm{H}$ HSQC experiments were measured for each step of titration.

Experiments were performed in Bruker 800 DRX, 700 Avance and $600 \mathrm{DRX} \mathrm{MHz}$ spectrometers at $30^{\circ} \mathrm{C}$. The data sets were processed and analyzed using the NMRPipe/NMRDraw software package. 


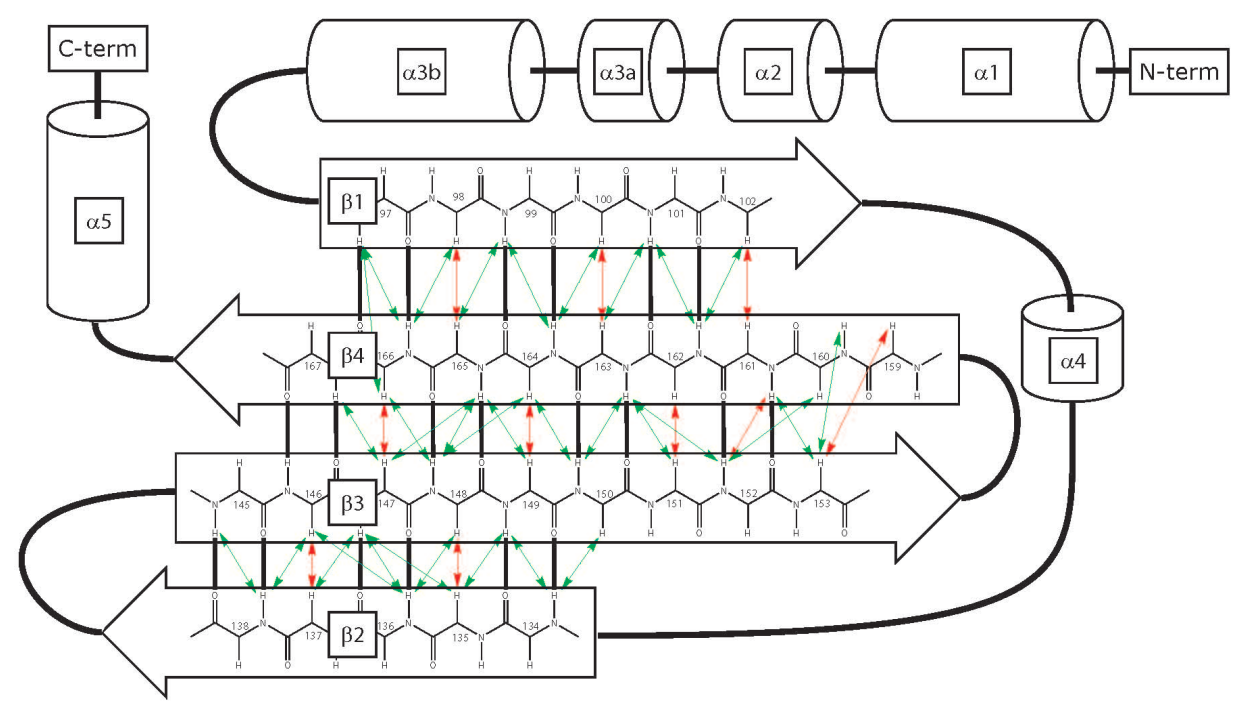

Figure 4.6: Secondary structure of DcuS-PD together with the intra-strand NOEs from which the topology of the $\beta$-strands were derived.

\subsubsection{Results and discussions}

The NMR solution structure of DcuS-PD without bound C4 dicarboxylates was determined. For the final refinement of the structure, four types of RDCs were measured and included in the structural calculations. Detail description of the structure follows.

\subsubsection{Structure of DcuS-PD}

\section{Secondary structure of DcuS-PD}

The secondary structure of DcuS-PD (Figure 4.6) consists of a long N-terminal $\alpha$-helix $\left(\alpha_{1}\right)$ ranging from amino acid 46 to 64 with a continuation from 68 to $72\left(\alpha_{2}\right)$. After a short loop there is another $\alpha$-helix $\left(\alpha_{3 a}: 77-79\right)$ and $\left(\alpha_{3 b}: 83-92\right)$ that is connected to the first $\beta$-strand $\left(\beta_{1}: 97-102\right)$ of the four stranded anti parallel $\beta$-sheet. $\beta_{1}$ is connected via an $\alpha$-helix $\left(\alpha_{4}\right.$ : 126-128) and a long loop to the second $\beta$-strand ( $\beta_{2}:$ 134-138), which is connected by a short loop to the third strand $\left(\beta_{3}: 145-153\right)$. Yet, another turn connects to the fourth $\beta$-strand $\left(\beta_{4}:\right.$ 159-167). From this strand the C-terminal helix follows after 
a short helix $\left(\alpha_{5}: 174-179\right)$. The secondary structural elements have been established by secondary chemical shift and characteristic sequential NOEs and connectivity of the four stranded anti parallel $\beta$-sheet by $\mathrm{H}^{N}, \mathrm{H}_{\alpha}$ and $\mathrm{H}_{\alpha}, \mathrm{H}_{\alpha}$ cross strand NOEs (Figure 4.6).

\section{Structure determination and discussion}

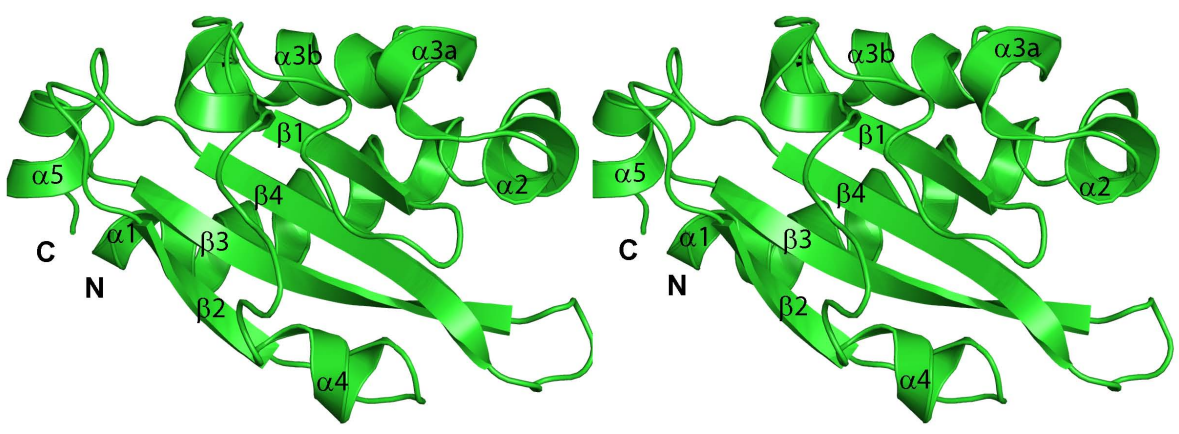

Figure 4.7: Stereo view of the NMR solution structure of periplasmic domain of DcuS. It reveals a PAS fold with $4 \beta$ strands and 5 main $\alpha$ helix. The structure is a $\alpha, \beta$-fold where both sides of the large $\beta$-sheet form hydrophobic cores with $\alpha$-helices and the long connector between helix $\alpha 4$ and strand $\beta 2$. The C-terminal helix is not fixed by NOEs and shows only small dipolar couplings, in agreement with a flexible helix.

Final refinement of the structure was done with 382 RDCs. These include $107{ }^{15} \mathrm{~N}^{-1} \mathrm{H}^{N}, 95$ ${ }^{1} \mathrm{H}_{\alpha^{-}}{ }^{13} C_{\alpha}, 114{ }^{15} \mathrm{~N}-{ }^{13} \mathrm{C}^{\prime}$ and $66{ }^{13} \mathrm{C}^{\prime}-{ }^{13} C_{\alpha}$ coupling. ${ }^{1} \mathrm{~J}{ }_{C \alpha H \alpha}$ couplings were also used to define $\phi$ angles. $\phi$ is negative for ${ }^{1} \mathrm{~J} \mathrm{C}_{\alpha \mathrm{H} \alpha}>137 \mathrm{~Hz}$ and in the $\alpha$-helix range for ${ }^{1} \mathrm{~J}\left(\mathrm{C}_{\alpha}, \mathrm{H}_{\alpha}\right)>145 \mathrm{~Hz}$ [129]. The structure is well ordered in the region 46-168, while the first few residues in the $\mathrm{N}$ and $\mathrm{C}$ terminal are not well ordered. The RDCs in these region are also small compared to the rest of the residues in the structure. The Het-NOE values in these regions of the protein are also relatively small, indicating dis-orderness in these parts of the protein. Figure 4.7 shows the mean structure derived from the 10 structures with the lowest energy.

The structure is a novel $\alpha, \beta$-fold completely dissimilar of the four helix bundle structure of the aspartate sensor [130, 131]. Consistent with gel shift data, the average $\mathrm{T}_{1 \rho}$ of the wild type protein is around $110 \mathrm{mS}$ corresponding to a monomer. This is dissimilar to the aspartate sensor which was a dimer in solution. In a DALI [45] search the closest match is photoactive yellow protein(PYP) from Halorhodospirahalophila [120], which also shows an $\alpha, \beta$-fold, however, with 5 instead of $4 \beta$-strands. The topology of strands $\beta_{3}, \beta_{4}$ and $\beta_{5}$ of PYP is 

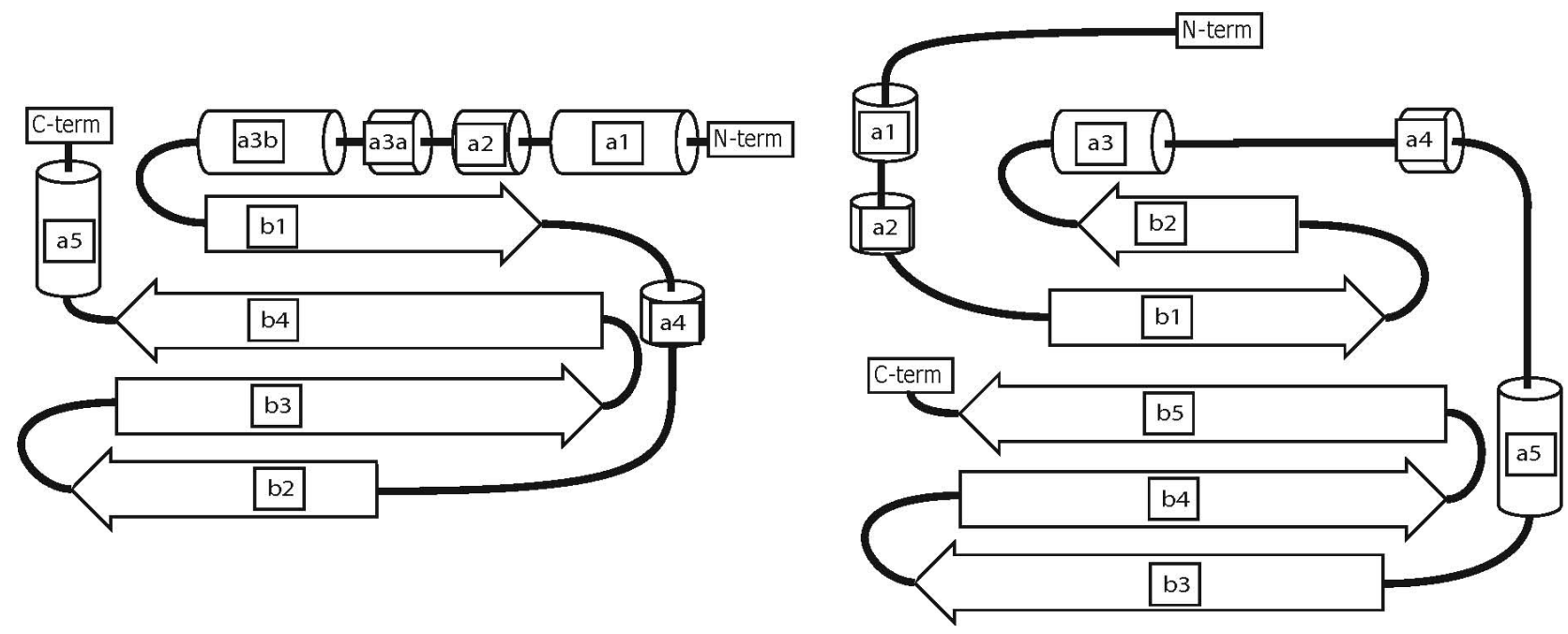

DcuS

PYP

Figure 4.8: Comparison of secondary structure of periplasmic domain of DcuS with its structure homologue, photo active yellow protein (PYP).

similar to the strands $\beta_{2}, \beta_{3}$ and $\beta_{4}$ of DcuS-PD (see Figure 4.8 for comparison of secondary structural element). However, the rest of the secondary structure is quite dissimilar. While in PYP the PAS core domain connects the strands $\beta_{2}$ and $\beta_{3}$ by crossing the whole $\beta$-sheet in a diagonal manner, there is no PAS core domain in DcuS-PD and the connection between sheets $\beta_{1}$ and $\beta_{2}$ is achieved on one side of the $\beta$-sheet. Similar to PYP, thee are two hydrophobic cores on both sides of the $\beta$-sheet formed. Helices $\alpha_{1}$ and $\alpha_{3 b}$ bind to the bottom side of the $\beta$-sheet, while helix $\alpha_{4}$ and the connector attach to the upper half of the $\beta$-sheet. In PYP the chromophore binding site is formed by the PAS core domain. Dissimilar to PYP, in DcuS-PD residues located in the $\beta$-sheet (Arg147) as well as in the connector (Arg107 and His110) across the $\beta$-sheet contribute to the putative binding site of fumarate. 


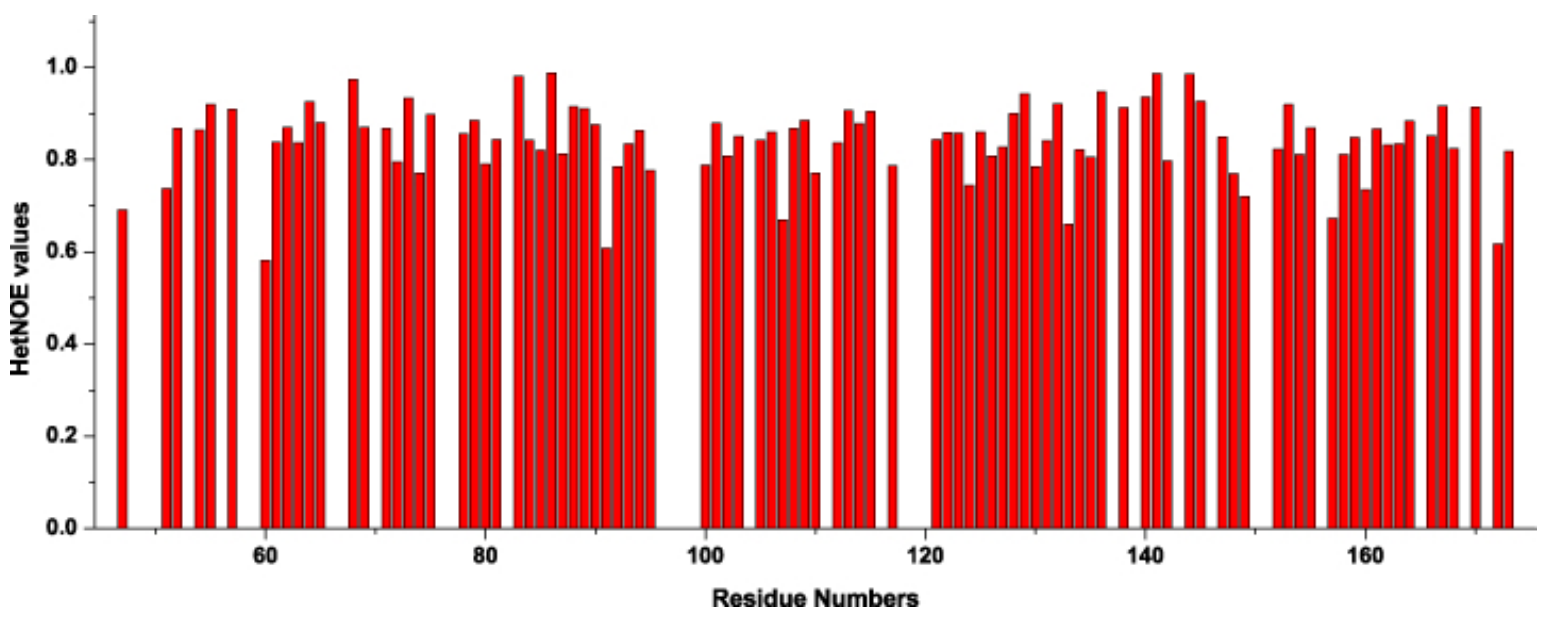

Figure 4.9: ${ }^{15} \mathrm{~N}-{ }^{1} \mathrm{H}$ Heteronulcear NOE values for DcuS-PD are plotted against residue number. Most of the values are between 0.6-0.9 indicating highly rigid structures. Het-NOE values for a number of residues in the $\mathrm{N}$ and $\mathrm{C}$ terminals were unable to be determined due to poor sensitivity in the reference spectra.

\subsubsection{Binding studies on DcuS-PD}

DcuS senses a number of C4-dicarboxylates like fumarate (Kd $5 \mathrm{mM})$, succinate etc [122]. The isolated DcuS-PD was used for the binding studies of the effectors to the domain by NMR spectroscopy. During the addition of fumarate, sharpening of peaks were observed for some of the amino acids of the periplasmic domain in ${ }^{15} \mathrm{~N}-{ }^{1} \mathrm{H}$ HSQC spectra (Figure 4.10). The affected residues cluster in a well defined region in the structure of the periplasmic domain of DcuS involving amino acid residues 107-168 (Figure 4.12 Left panel). This region in the structure correspond to the binding pocket of citrate in the periplasmic domain of CitA. There were no chemical shift changes observed during the titration. Sharpening of peaks were not observed when the protein sample contained a detergent (CHAPS) used for the titration with fumarate. This suggest that the detergent might have shielded the binding pocket of DcuS-PD. 


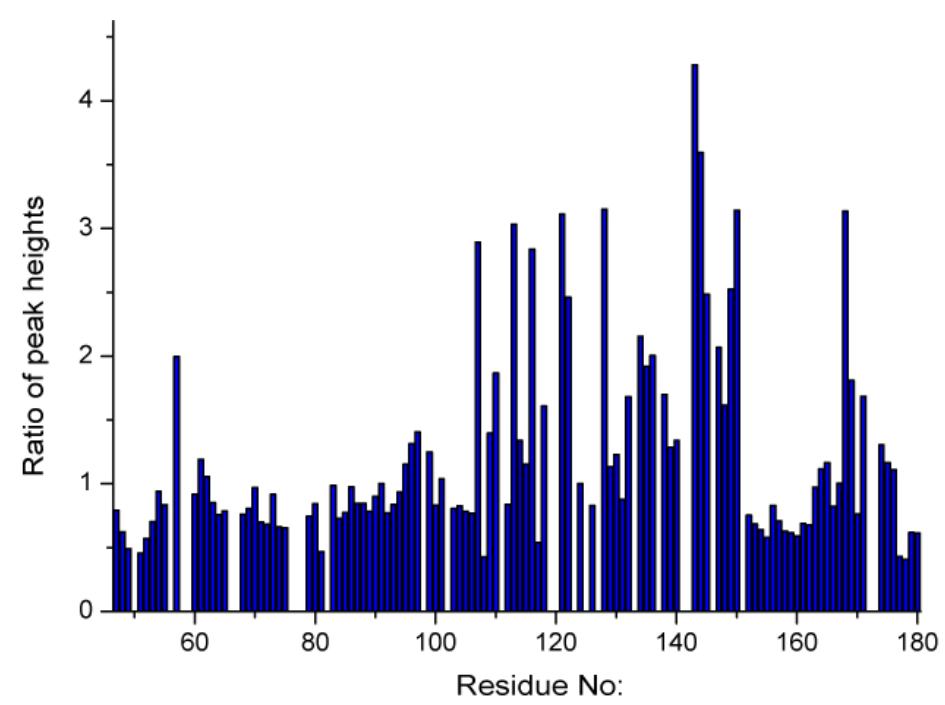

Figure 4.10: Ratio of peak heights in ${ }^{15} \mathrm{~N}-{ }^{1} \mathrm{H}$ HSQC spectrum after the addition of 30 -fold excess of fumarate to DcuS-PD. Titrations were carried out with addition of properly buffered sodium fumarate to a solution of protein at $1.2 \mathrm{mM}$. Residue that shows the maximum peak intensity is Q144

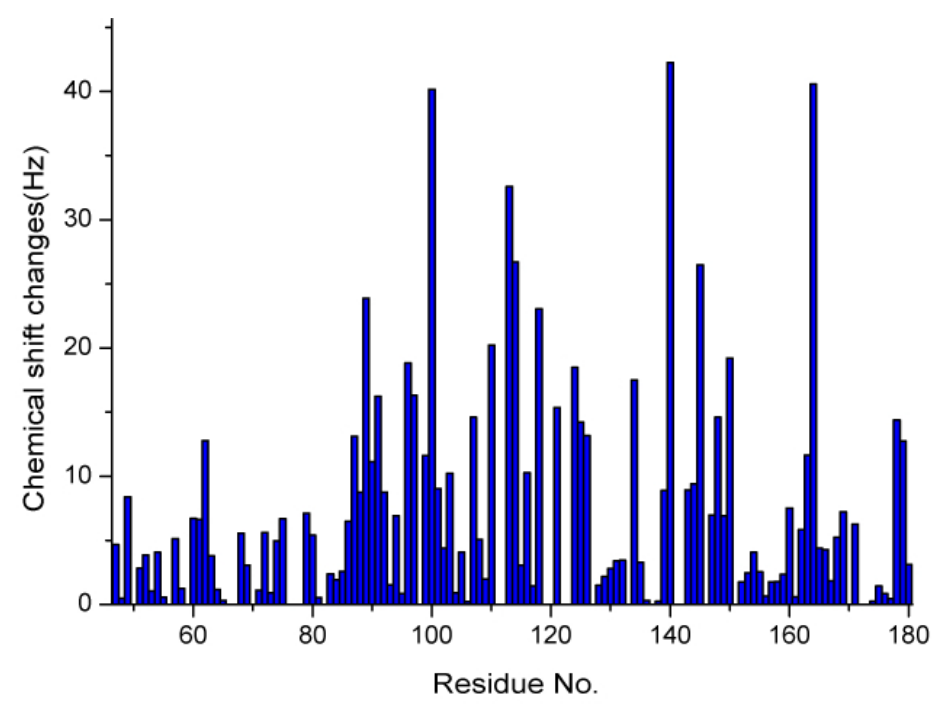

Figure 4.11: Plot of chemical shift changes in ${ }^{15} \mathrm{~N}-{ }^{1} \mathrm{H}$ HSQC when D-tartrate (50-fold excess) is added to periplasmic domain of DcuS $(1.2 \mathrm{mM})$. The chemical shift plotted here is the sum of ${ }^{15} \mathrm{~N}$ and ${ }^{1} \mathrm{H}^{N}$ chemical shift, properly scaled using the method given in chapter 2, section 2.2.9. Residue that shows the maximum chemical shift change is G140. 


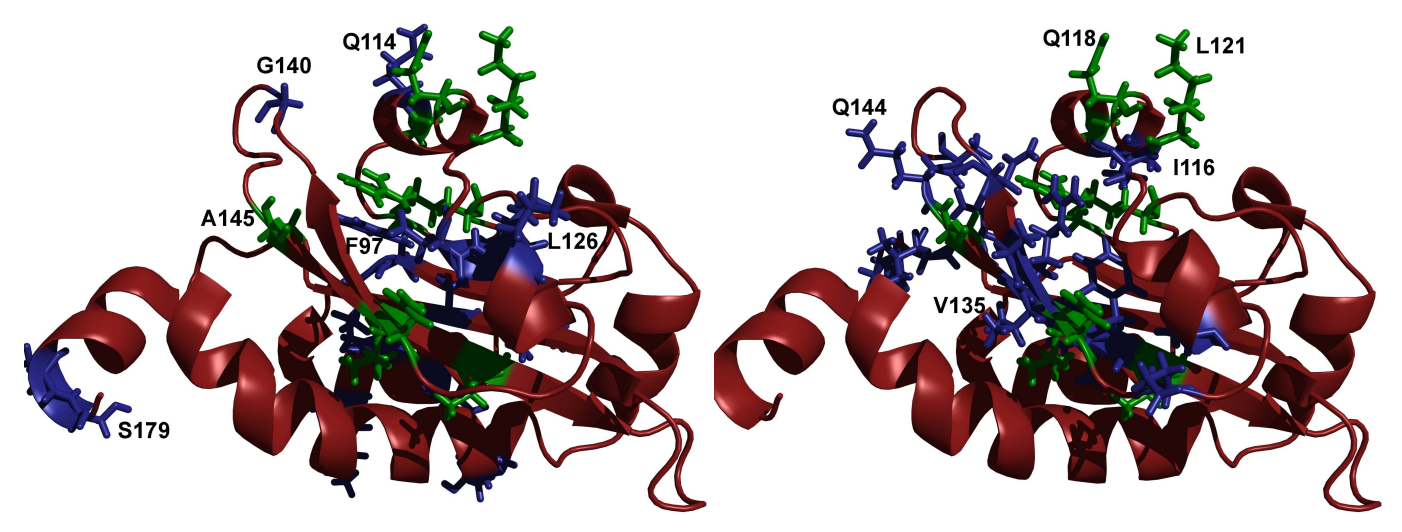

Figure 4.12: (Right) Structure of DcuS periplasmic domain showing the residues most affected by fumarate titration. The residues, represented as sticks in the structure are R107, A113, I116, K121, A128, N134, A136, A143, Q144, A145, R147, F149 and T150. (Left) Structure of DcuS periplasmic domain showing the residues most affected by the tartrate titration. The residues shown as sticks on the structure are V89, K91, L96, F97, V100, H110, A113, Q114, Q118, K121, D124, N134, G140, A145, and T150. Common residues that are affected both by fumarate and tartrate titration are represented in green colour and the residues individually affected by the ligand titration are shown in blue sticks.

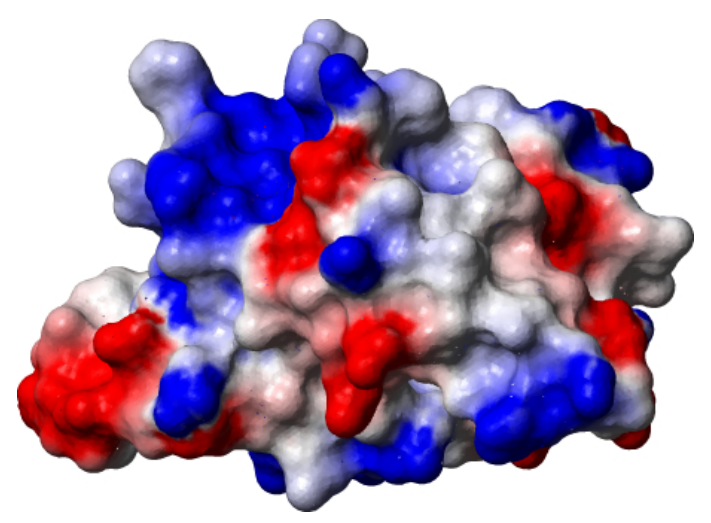

Figure 4.13: Electrostatic surface potential of DcuS-PD with positive and negative potentials coloured blue and red respectively. This reveals a positive charged surface region in the putative binding site of DcuS-PD. Residues of this region of the protein are mainly affected by the ligand titration. 
D-tartrate is regarded as a non-physiological stimuli of DcuS [132], but is structurally closely related to other $\mathrm{C} 4$ di-carboxylates and binds to DcuS with a higher affinity than fumarate (apparent $\mathrm{Kd}$ is $0.5 \mathrm{mM}[132]$ ). Chemical shift changes were observed for a number of residues when DcuS-PD was titrated with D-tartrate (Figure 4.11). Most of these residues belong to the same region that was affected by fumarate binding. Such chemical shift changes were observed only for D-tartrate and not for L-tartrate. There was a set of common residues that were affected both by the fumarate and tartrate titration (Ala113, Lys121, Asn134, Ala145 and Thr150-refer Figure 4.12 : Residues coloured in green). Other residues were specifically affected by fumarate (Arg107, Ile116, Ala128, Ala136, Ala143, Gln144, Arg147 and Phe149-refer Figure 4.12 Residues coloured in red in the right panel) or D-tartrate addition (Val89, Lys91, Leu96, Phe97, Val100, His110, Gln114, Gln118, Asp124 and Gly140refer Figure 4.12 Residues coloured in blue in the left panel). A comparison of the affected residues reveal that fumarate and tatrate bind to the same positively charged binding pocket in the DcuS-PD domain (Figure 4.13). In addition, some residues located outside this defined binding pocket are specifically affected by D-tartrate and more non-polar residues are affected by fumarate than D-tartrate, the latter containing two additional hydroxyl groups. Titration experiments were also performed with nitro-propionate (known to be sensed by DcuS at higher affinity than fumarate, Kd $0.4 \mathrm{mM}$ [132]) as ligand, but unfortunately no visible effects were observed in the spectrum during the titration.

Alignment of the amino acid sequence of the periplasmic domain of DcuS from E.Coli with those from the citrate sensor CitA from Klebsiella pneumoniae 127, 133] and other DcuS and CitA proteins revealed conserved residues in the members of the CitA/DcuS family (Figure 4.14). Most of the conserved residues are hydrophobic and might be structurally relevant. Charged residues are conserved in both types of proteins (D102, R107, H110 and R147).

Mutation of the charged residue like R147, H110 to a non polar residue like Alanine completely repress dcuB'-'lacZ reporter gene fusion, indicating loss of effector binding. Another 
set of mutation for amino acid residues that are specifically conserved in DcuS-type proteins (M103, F120, F149 and Q159) also impair DcuB'-'lacZ expression [132].

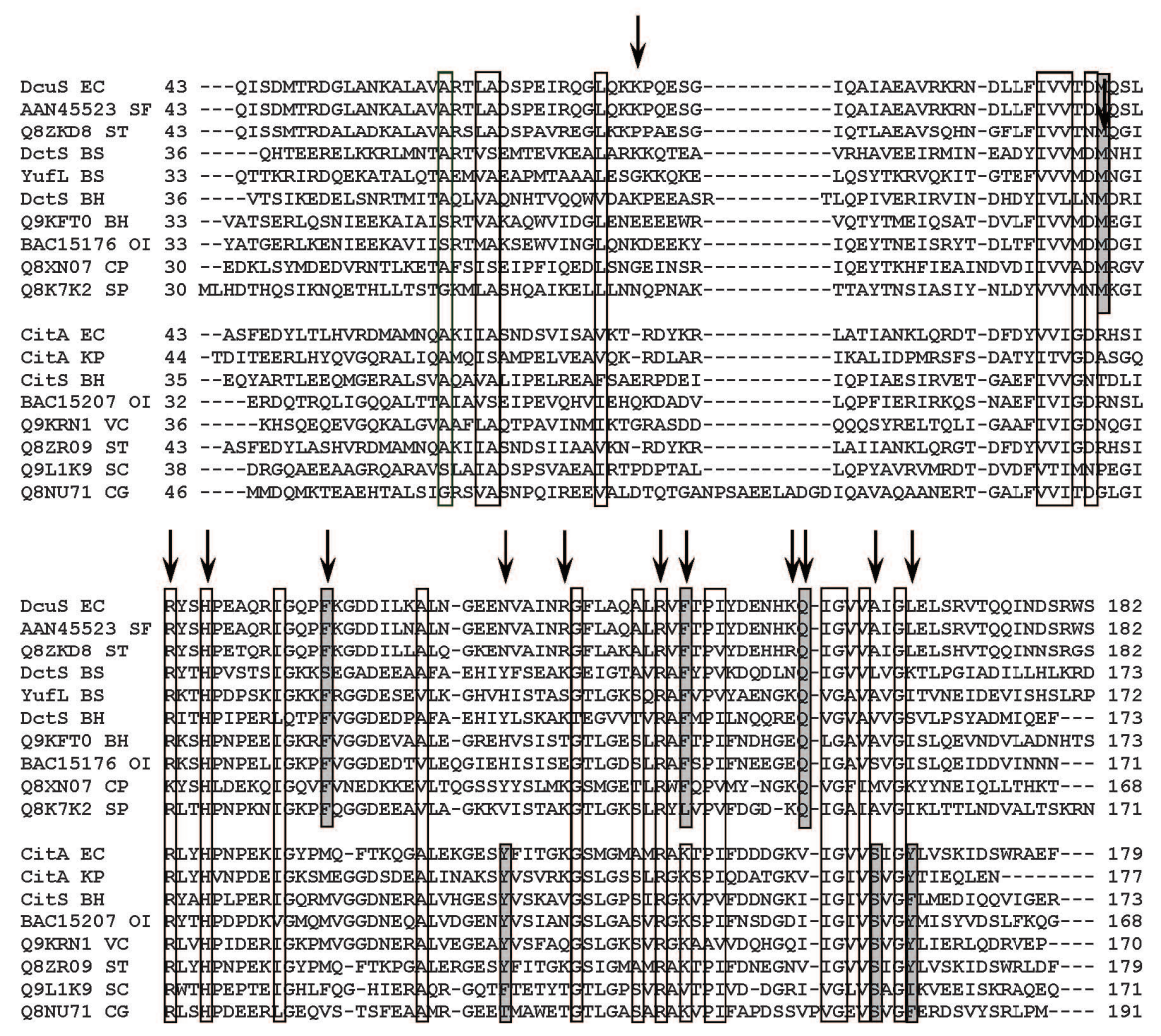

Figure 4.14: Comparison of the amino acid sequences of the periplasmic sensor domains of C4dicarboxylate or citrate sensory histidine kinases. Amino acid residues conserved in both types of proteins are boxed; amino acids conserved in only one of the types of are boxed and shaded. Amino acid residues of E. coli DcuS that were changed by site-directed mutagenesis are indicated by arrows. BS, Bacillus subtilis; BH, Bacillus halodurans, CG, Corynebacterium glutamicum; CP, Clostridium perfringens; EC, Escherichia coli; KP, Klebsiella pneumoniae; OI, Oceanobacillus ihejensis; SC, Streptomyces coelicolor; SF, Shigella flexneri; SP, Streptococcus pyogenes; ST, Salmonella typhimurium; VC, Vibrio cholerae. This figure has been reproduced from [132].

The wild type periplasmic domain used in the study was well folded (See Figure 4.15 peaks in blue) and the residues R107, H110, and R147, found from the mutational studies to be essential for fumarate binding appear to be in close proximity in the structure, suggesting that the binding motif is retained in the periplasmic domain. In order to observe the influence of mutation on the effector binding on DcuS-PD, ${ }^{15} \mathrm{~N}-{ }^{1} \mathrm{H}$ HSQC spectra of the mutants were measured. ${ }^{15} \mathrm{~N}-{ }^{1} \mathrm{H}$ HSQC spectra of the mutants H110A and F120M showed very small 
chemical shift dispersion in proton dimension which are characteristic of unfolded proteins ( Figure 4.15 panel B and C). For R147A, severe line broadening of resonances were observed denoting a high oligomerization state of the protein( Figure 4.15 panel D). Moreover R147APD was highly unstable and precipitated in the NMR tube very quickly. Unfolding and aggregation of these mutants indicate a loss of structure and stability in these mutants. This study illustrates that these residues are very important for the fold of DcuS-PD. The decrease in the binding affinity of ligands in the biological assay could partly be due to these point mutations which are related to the loss of the three dimensional structure of mutated sensory domain.

\subsubsection{Conclusion}

Solution structure of the ligand free periplasmic domain of DcuS was determined. Structure reveals a PAS fold with the putative binding site of the ligand formed by the beta sheet and the inter strand loops. Titration experiments were carried out with dicarboxylates namely, fumarate and tartrate. The affected residues cluster around the putative binding pocket of DcuS-PD. Upon fumarate titration only sharpening of peaks were observed indicating a stabilization of the structure with the binding of fumarate. Upon titration with the nonphysiological stimuli tartrate, small chemical shift changes were observed for a subset of peaks in the HSQC spectrum. The presence of two hydroxyl group in tartrate makes this C4 di-carboxylate to bind to DcuS-PD with much higher affinity. Because of the presence of two more charges, more polar residues are affected upon tartrate titration. However, even with tartrate, no major conformational changes could be observed in the C-terminus of the protein to indicate signal transduction. 

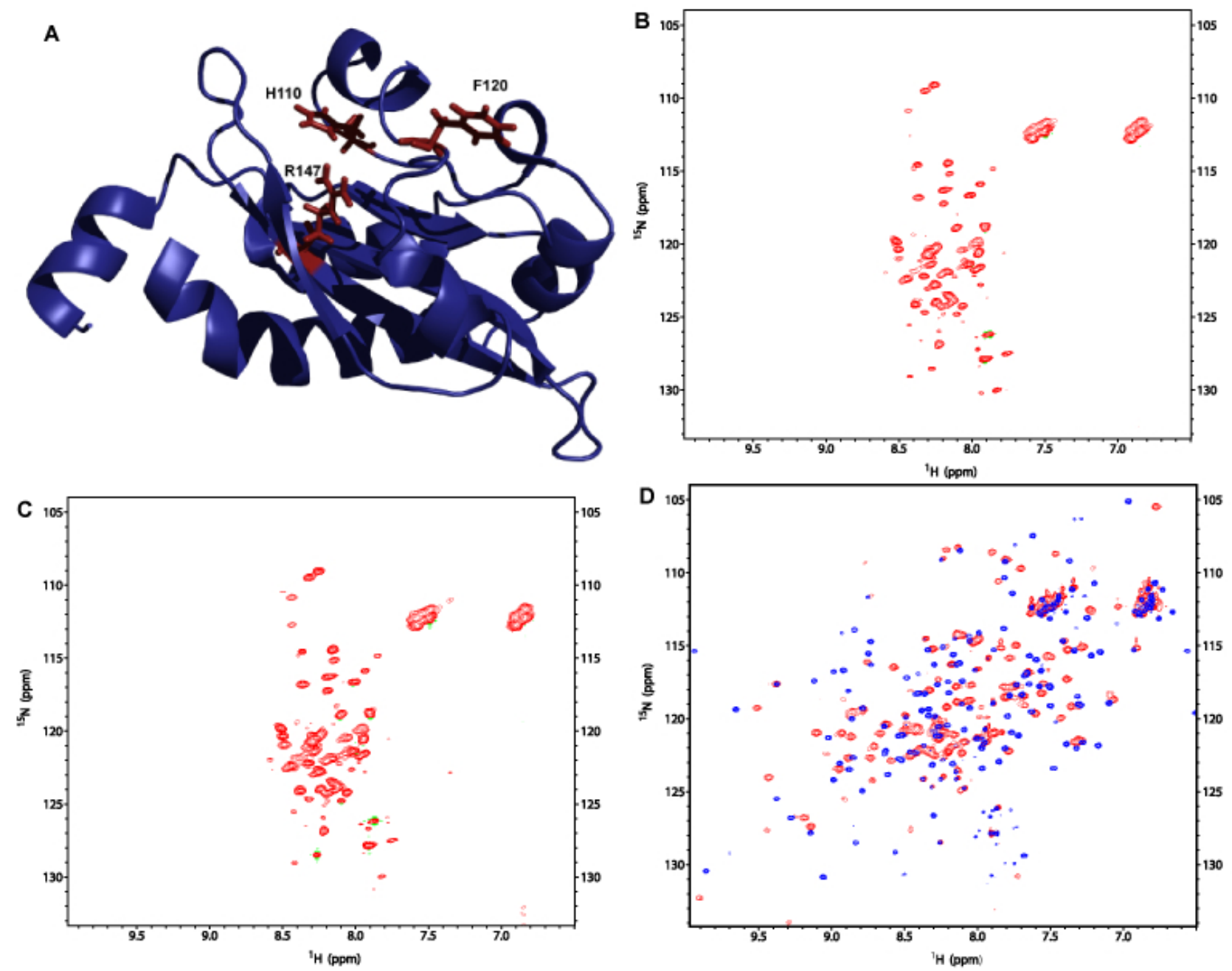

Figure 4.15: (A) DcuS periplasmic domain with the residues that are mutated are indicated by sticks. The residues shown are H110, F120 and R147. (B) ${ }^{15} \mathrm{~N}^{1}{ }^{1} \mathrm{H}$ HSQC of DcuS-PD mutant H110A. The protein was unfolded with small dispersion in chemical shift in the proton dimension.(C) ${ }^{15} \mathrm{~N}-{ }^{1} \mathrm{H}$ HSQC of DcuS-PD mutant F120A. This protein is also unfolded with small dispersion in chemical shift in the proton dimension. (D) Overlay of ${ }^{15} \mathrm{~N}-{ }^{1} \mathrm{H}$ HSQC of wild type DcuS periplasmic domain (blue) with R147A mutant of DcuS-PD (red). Severe line broadening is observed for the peaks of the mutant in the HSQC. Large number of peaks were missing when compared to peaks in the HSQC of the wild type DcuS-PD. 


\subsection{Periplasmic domain of the sensory domain of the two component citrate sensor CitA}

\subsubsection{Introduction}

Several species of enterobacteria are able to use citrate as the sole carbon as energy source in both aerobic and anaerobic conditions. The most extensively studied organism with respect to citrate metabolism is Klebsiella pneumoniae. During aerobic growth, citrate is taken up in symport with protons and then metabolized via the tri-carboxylate cycle. The transport is catalysed by the a protein encoded by the citH gene, which is expressed constitutively in the presence of oxygen [134, 135, 136]. During anaerobic growth, uptake of citrate is a $\mathrm{Na}^{+}-$ dependent process [137] catalysed by the CitS protein. Within the cell, citrate is metabolized by the citrate specific fermentation enzymes like Citrate lyase, oxaloacetate decarboxylase etc.

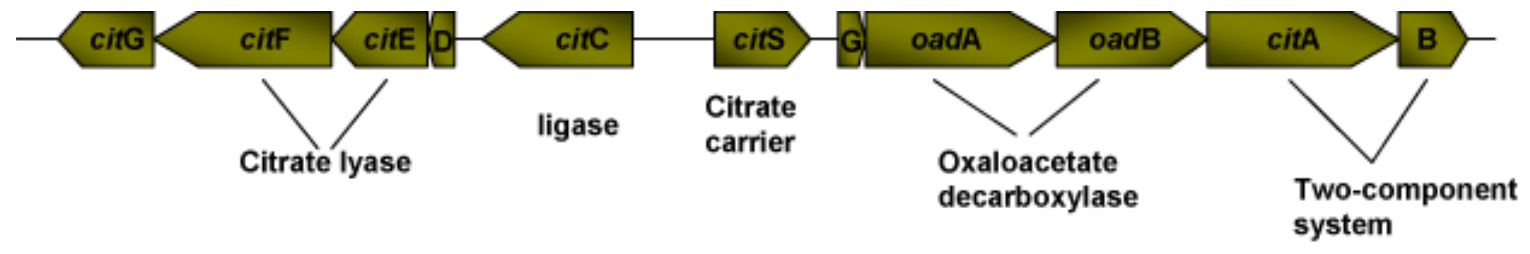

Figure 4.16: The cit regulon of Klebsiella pneumoniae. The regulator CitB binds between citC and citS, where it induces the transcription of the genes required for citrate fermentation.

The cluster of genes which confer the property for bacteria to grow anaerobically is believed to form a regulon [138] ( See Figure 4.16]). These include genes for the citrate carrier protein CitS [139], that is flanked downstream by the genes for oxaloacetate decarboxylase(oadGAB) [140, 141, 142] and upstream by the citCDEFG genes coding for citrate lyase ligase (CitC), citrate lyase (CitDEF) and a protein of yet unknown function (CitG) [143. The two component regulatory system citAB located downstream of the oadB gene codes for the sensor histidine kinase (CitA) and its response regulator (CitB) [138]. Mutational analysis suggest that the CitAB system is essential for the expression of citrate specific fermentation enzymes. CitB null mutants of Klebsiella pneumoniae were unable to grow 
anaerobically with citrate as the sole carbon source and none of the citrate specific fermentation enzymes were synthesized [138]. This showed that citS, oadGAB and citDEF required the CitB protein for expression and therefore are part of a regulon. From analogy to other two component systems [144, 124, 145], the signal trigger should then come from the sensor histidine kinase CitA [127]. In the presence of specific signal molecule(citrate), CitA is first autophosphorylated with ATP as substrate. CitA delivers the phosphoryl moiety to CitB. CitB then binds to a specific target within the cit regulon ( Figure 4.16), thereby inducing the transcription of its genes.

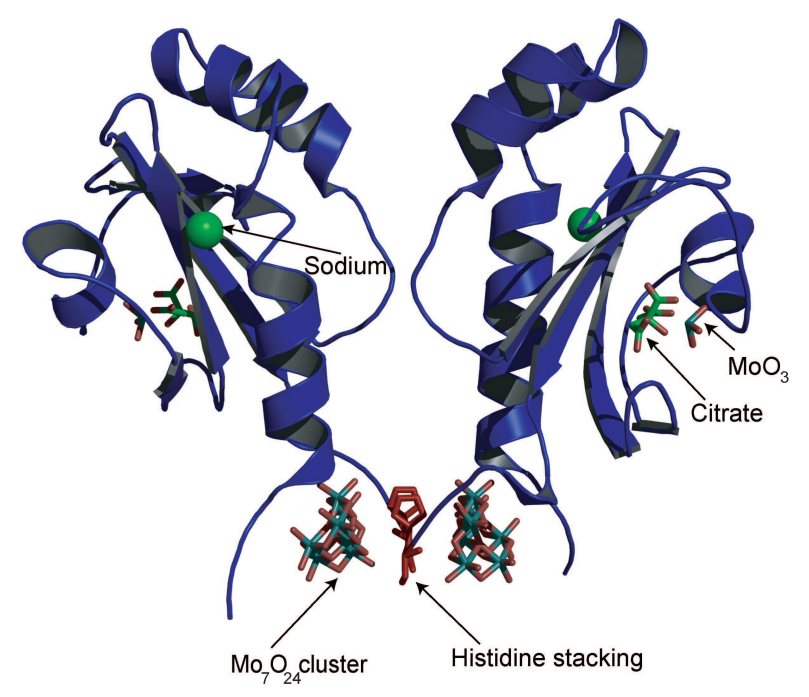

Figure 4.17: X-ray structure of GJ dimer of CitAP in complex with citrate and molybdate (pdb id 1P0Z). The citrate-binding pocket is on the right. Citrate-molybdate (right) and isopolymolybdate (bottom) groups are shown as stick figures. A putative sodium ion is shown as a green sphere

CitA, like DcuS is a membrane bound sensor kinase consisting of a periplasmic domain flanked by two trans-membrane helices, a linker domain and the conserved kinase domain. The structural morphology of CitA is also very similar to DcuS. As mentioned above, the signal detection happens in the periplasmic domain of CitA (hence forth called CitAP). CitAP is very specific for citrate [127] and bio-chemical studies indicate that citrate binds to CitAP with a $\mathrm{K}_{d}$ of $5 \mu \mathrm{M}[133$. The aim of the study was to investigate the structure of CitAP with and without the ligand and to determine the structural changes that may cause the signal transduction across the membrane into the cytoplasmic domain. 
X-ray structure of CitAP in complex with citrate and molybdate was already determined [146] (pdb id: 1P0Z, Figure 4.17). The structure reveals that CitAP adopts a PAS domain fold with the ligand binding pocket formed by the $\beta$ sheets, a short helix and the flanking random coil residues (Figure 4.17). Two arginines, a lysine and a histidine were found to interact with citrate in the binding pocket. Due to the crystallization condition, there was also a molybdate $\left(\mathrm{MoO}_{3}\right)$ moiety in the binding pocket which was in complex with citrate. The presence of molybdate in the binding pocket was unexpected as citrate is transported into the cell without any metal complex formation. Two dimeric forms of CitAP were observed in the crystal lattice. One of it might be artificially induced by the stacking of the C-terminal histidine tags, used for the purification of the protein, as seen in the Figure 4.17. A large molybdate cluster $\left(\mathrm{Mo}_{7} \mathrm{O}_{24}\right)$ was also found interacting with the $\mathrm{N}$ - and C-terminal region. The citrate free structure of CitAP was still undetermined and hence the structural change accompanying the ligand binding was poorly understood.

\subsubsection{Materials and Methods}

\section{Sample Optimization}

Initially, CitAP protein was produced in the lab of Prof. Bott in Jülich. The protein was first produced with a C-terminal 6 membered histidine tag. For testing the stability of the protein, two fragments of the protein were made, a longer fragment consisting of amino

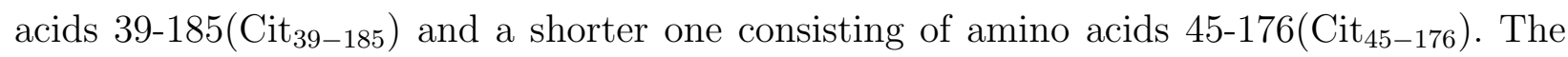
longer protein fragment included a small part of the trans-membrane helix in the $\mathrm{N}$ - and C-terminal. ${ }^{15} \mathrm{~N}-{ }^{1} \mathrm{H}$ HSQC spectrum of the ${ }^{15} \mathrm{~N}$ labeled protein produced from the two fragments are shown in the Figure 4.18. The longer fragment (panel (A) Figure 4.18) was unstable and precipitated very quickly. The HSQC spectrum of the longer fragment had more peaks than the one from the shorter fragment (panel (B) Figure 4.18) but most of the peaks were very broad. This could be due to the presence of hydrophobic residues in the longer construct (more specificaly tryptophan,leucine etc) resulting in an increased propensity to aggregate. The shorter fragment (more stable) was used for all further studies as the peaks in its ${ }^{15} \mathrm{~N}-{ }^{1} \mathrm{H}$ HSQC spectrum had more favorable line width for three dimensional experiments. 

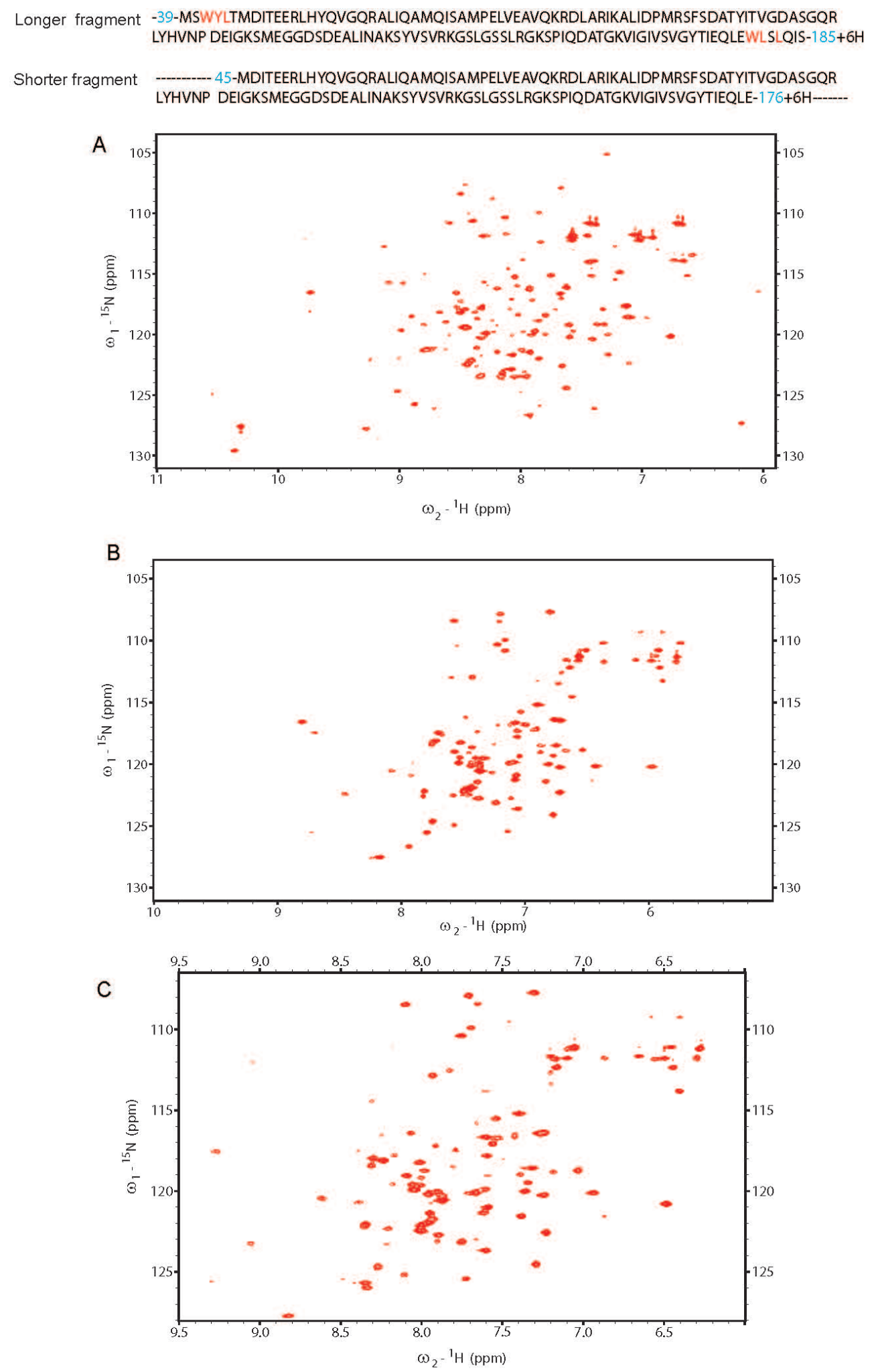

Figure 4.18: Amino acid residues for the short and long fragment of the protein prepared are given on the top. (A) ${ }^{15} \mathrm{~N}^{1}{ }^{\mathrm{H}}$ HSQC of longer construct. The spectrum was measured on the 600DRX spectrometer at $310 \mathrm{~K}$. (B) ${ }^{15} \mathrm{~N}-{ }^{1} \mathrm{H}$ HSQC spectrum of the shorter construct measured on the 600 DRX spectrometer at $310 \mathrm{~K}$. Only $62 \%$ of the expected peaks are seen in the spectrum. $(\mathbf{C}){ }^{15} \mathrm{~N}-{ }^{1} \mathrm{H}$ HSQC of CitAP of the shorter construct at higher salt concentratation $(300 \mathrm{mM})$. Fewer peaks are seen in this spectrum compared to the HSQC spectrum in (B) 
Nevertheless the shorter construct had only $60 \%$ of the expected peaks. Hence to further improve the spectrum of the smaller construct, different conditions for experiments were tried.

1) Change of $\mathrm{pH}$ of the buffer from 7 to 6 did not improve the spectrum. Lowering the $\mathrm{pH}$ reduces the amide-proton and water chemical exchange. This confers better relaxation property for NMR measurements. Hence all later experiments were measured at $\mathrm{pH} 6.00$

2) Salt titration was carried out with step-wise addition of properly buffered $\mathrm{NaCl}$ solution (panel (C) Figure 4.18). Again no improvement in the spectra were observed (0 to $100 \mathrm{mM}$ ). On addition of more salt, some resonances start to disappear (100 to $300 \mathrm{mM}$ ). All later experiments were measured with no additional salt other than the $50 \mathrm{mM}$ sodium present in the phosphate buffer.

3) To determine the optimal temperature for measurements, HSQC experiments were measured by varying temperature from $290 \mathrm{~K}$ to $310 \mathrm{~K}$. Better spectra were observed at higher temperatures.

The final buffer condition were $50 \mathrm{mM} \mathrm{Na} 2 \mathrm{HPO}_{4} / \mathrm{NaH}_{2} \mathrm{PO}_{4}$ at $\mathrm{pH}$ 6.00. The spectrum also improved when the histidine tag on the protein was cleaved off. The conditions optimized for the histidine tagged protein were later used for all experiments measured for preparations without the histidine tag. All three dimensional experiments were measured at $298 \mathrm{~K}$ using the protein prepared with the shorter construct and without the histidine tag. At higher temperatures the protein started to precipitate in the course of longer experiment. The triple resonance experiments measured for the backbone assignment are listed in the table 2.2 and 2.3 . The citrate bound form of CitAP was obtained by the step wise addition of sodium citrate to the citrate free sample of CitAP. Three dimensional experiments for the backbone assignment were repeated for the citrate bound form of CitAP. These samples were stable for a week and then degraded very quickly.

Later, ${ }^{15} \mathrm{~N},{ }^{13} \mathrm{C}$ labeled protein samples were produced in our department in the molecular biology laboratory, supervised by Dr. Stefan Becker. Samples were very stable and did not degrade even after keeping them at $4^{\circ} \mathrm{C}$ for months. These samples were used to re-measure the three dimensional experiments acquired with the previous sample. The measurements 
were also repeated for the citrate bound form of CitAP. Because of the higher stability of the samples all three dimensional experiments were measured at $310 \mathrm{~K}$. These experiments were used for the protein backbone assignments of citrate free and citrate bound form of CitAP.

In order to measure RDCs, citrate free and bound form of CitAP samples were oriented in bacterial filamentous phages. The concentration of the phages in the sample were 12 $\mathrm{mg} / \mathrm{ml}$ in the citrate free and $15 \mathrm{mg} / \mathrm{ml}$ in the citrate bound CitAP. ${ }^{1} \mathrm{D}_{N H}$ couplings were measured for the citrate bound form of CitAP. A set of ${ }^{1} \mathrm{D}_{N H},{ }^{1} \mathrm{D}_{N C}$ and ${ }^{1} \mathrm{D}_{C C \alpha}$ were measured for citrate free form of CitAP. The ${ }^{1} \mathrm{D}_{N C O}$ and ${ }^{1} \mathrm{D}_{N H}$ couplings were measured using the TROSY-HNCO sequence described in chapter $3 .{ }^{1} \mathrm{D}_{C^{\prime} C \alpha}$ couplings were measured with $\mathrm{HNCO}$ experiment by removing $\mathrm{C}_{\alpha}$ decoupling in the $\mathrm{C}^{\prime}$ dimension. The measured residual dipolar couplings of CitA of the free and bound form are given in the Table B.6 and B.5 respectively.

Simultaneous ${ }^{15} \mathrm{~N}$ and ${ }^{13} \mathrm{C}$ NOESY experiment was measured with a mixing time of 120 ms. Heteronuclear NOE experiments were measured with a recycling delay of 5 seconds. The average rotational correlation time $\left(\tau_{c}\right)$ of CitAP was determined using the NMR experiment given in reference [64]. A detailed description of the calculations of $\left(\tau_{c}\right)$ is given in the methods part (Chapter 2, section 2.2.8.

\subsubsection{Results and Discussion}

\section{Titration results}

As indicated earlier, the available structure of CitAP(1P0Z) was obtained by crystallizing a histidine tagged protein. The structure was found to contain citrate in complex with molybdate.

In order to determine the influence of molybdate on the binding of citrate to His-tagged CitAP, titration experiments were carried out with citrate and molybdate using histidine tagged ${ }^{15} \mathrm{~N}$ labeled CitAP. ${ }^{15} \mathrm{~N}-{ }^{1} \mathrm{H}$ HSQC experiment was measured for each titration step. Titrations were carried out by step-wise addition of sodium citrate to the protein. Sharpening 
of peaks and chemical shifts changes were observed in the ${ }^{15} \mathrm{~N}-{ }^{1} \mathrm{H}$ HSQC with an addition of one equivalent of citrate to the protein, indicating the binding of citrate to CitAP (Figure 4.19 peaks in blue). A second sample was used to add sodium molybdate to the protein. Broadening of signals were observed indicating a higher aggregation state of the protein. Most of the signals disappeared upon addition of 20 fold molar excess of molybdate (Figure 4.19 peaks in green ). On addition of citrate to the molybdate bound CitAP solution, line broadening decreased and peaks started to re-appear. No major changes were obtained after addition of 20 fold excess of citrate to the molybdate bound CitAP solution (Figure 4.19 peaks in red). This corresponds to the published crystallization condition of citrate bound CitAP (1P0Z). The difference in the HSQC spectra of citrate bound CitAP and citrate and molybdate bound-CitAP, demonstrates that there might be a difference in the protein structure with citrate alone and citrate and molybdate together bound to CitAP. From the titration experiments it is clear that the citrate binding to CitAP does not require the presence of molybdate. Since the importance of molybdate in signal transduction is less understood, further experiments for backbone assignments were obtained without the influence of molybdate.

Therefore the new study of CitAP will be based on the backbone assignment of the CitAP shorter fragment, without the histidine tag, in a buffer which does not contain any molybdate.

\section{Extent of assignment}

Usual three dimensional experiments were acquired for the backbone assignment of the citrate free and citrate bound CitAP. These are listed in Table 2.4. Only 85 peaks were observed out of 129 peaks expected in the ${ }^{15} \mathrm{~N}^{-1} \mathrm{H}$ HSQC spectrum of citrate free CitAP. The use of TROSY based NMR experiments did not give any additional peaks. Out of the 80 peaks for which $\mathrm{C} \alpha$ connectivities were present, 72 peaks were assigned. The Backbone chemical shifts of the assigned residues are given in Table B.1. The ${ }^{15} \mathrm{~N}-{ }^{1} \mathrm{H}$ HSQC spectrum of the citrate bound form of CitAP gave 109 peaks out of the possible 129 peaks expected from the amino acid sequence. Since the binding of citrate to CitAP was in slow exchange, peaks could not be followed during the titration. Hence the three dimensional experiments for 


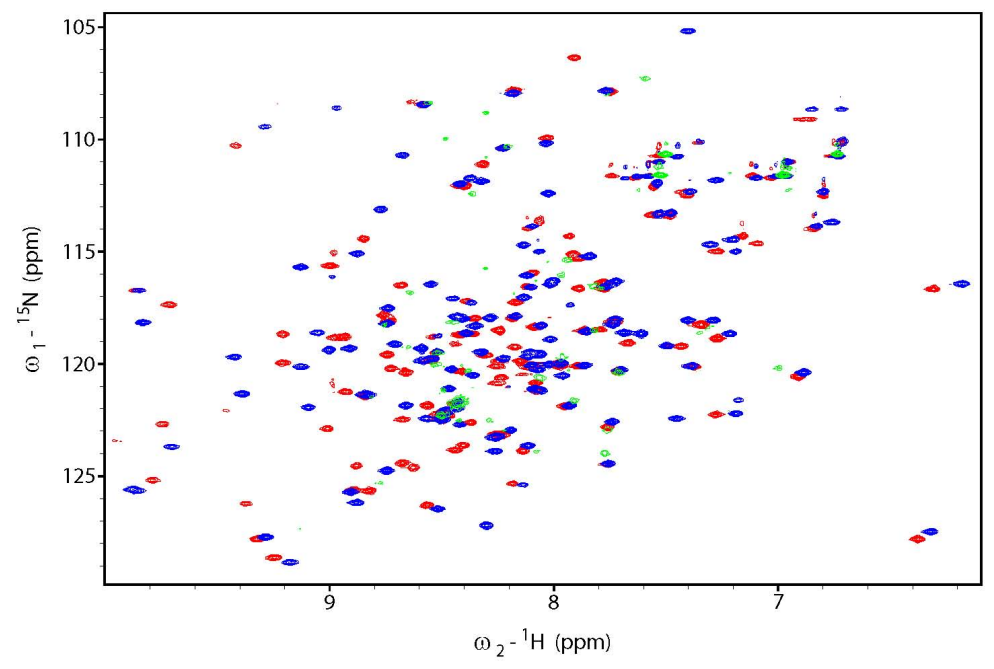

Figure 4.19: Overlay of ${ }^{15} \mathrm{~N}-{ }^{1} \mathrm{H}$ HSQC spectra of molybdate titration using the His-tagged CitAP shorter construct. Peaks of the spectrum with 20 fold excess of molybdate is in green. Extreme line broadening is observed for most of the peaks. Peaks in red were obtained from a spectrum with 20 fold excess of citrate and molybdate. Peaks in blue were obtained with 5 fold excess of citrate in the protein sample. Large changes in chemical shifts are seen between the spectrum of molybdate and citrate, and citrate alone.

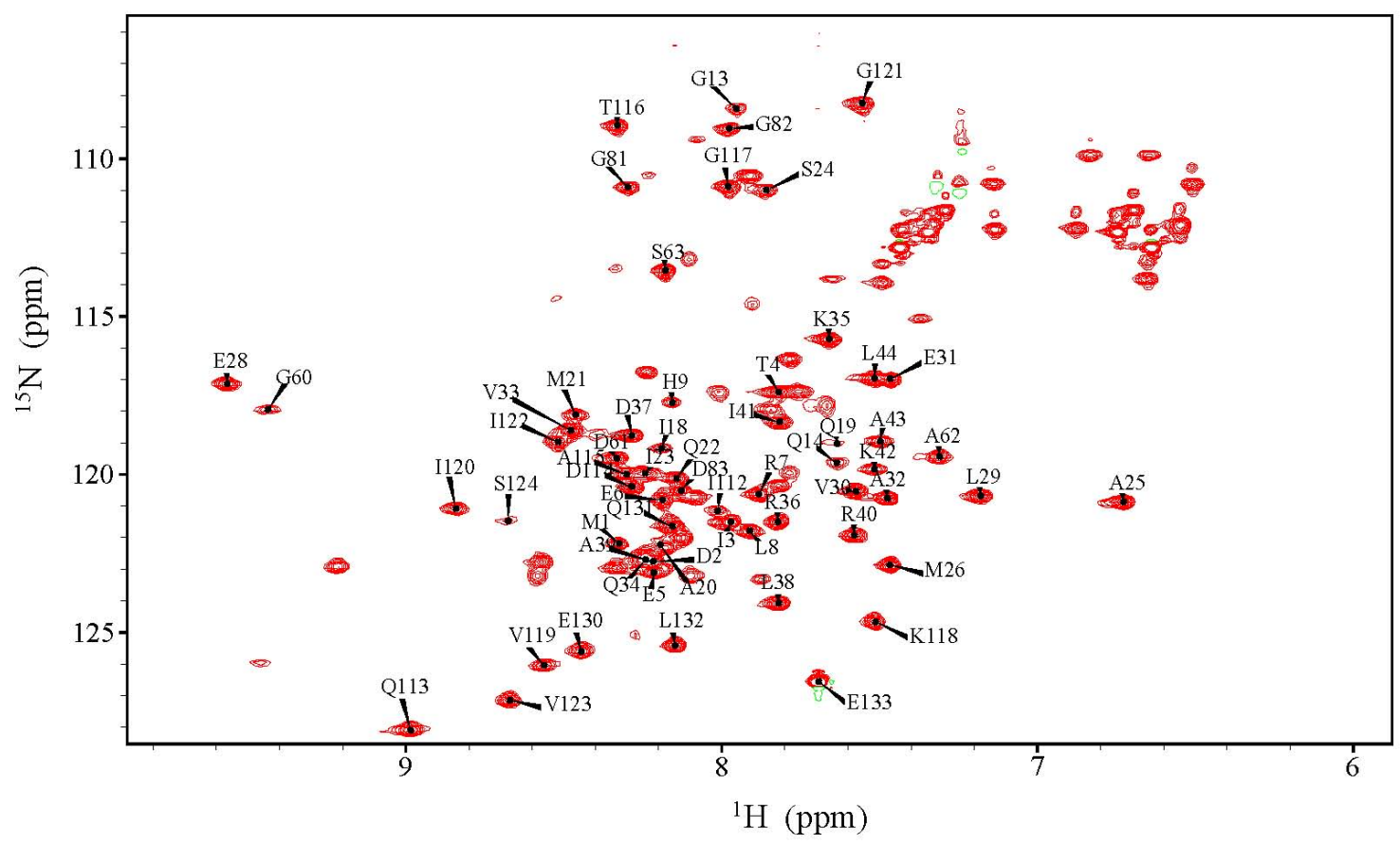

Figure 4.20: ${ }^{15} \mathrm{~N}-{ }^{1} \mathrm{H}$ HSQC spectrum of citrate free CitAP with the assignment of amino acid given on top of the peaks. The spectrum was measured at $900 \mathrm{MHz}$ at $310 \mathrm{~K}$. 
backbone assignments had to be re-measured for the citrate bound form of CitAP (Table 2.5). Assignment was obtained for 100 out of 109 peaks seen in the ${ }^{15} \mathrm{~N}-{ }^{1} \mathrm{H}$ HSQC spectrum. Chemical shifts of the assigned peaks are listed in Table B.2. ${ }^{15} \mathrm{~N}-{ }^{1} \mathrm{H}$ HSQC of the citrate free and citrate bound forms of CitAP including their assignments are given in the Figure 4.20 and Figure 4.21 respectively.

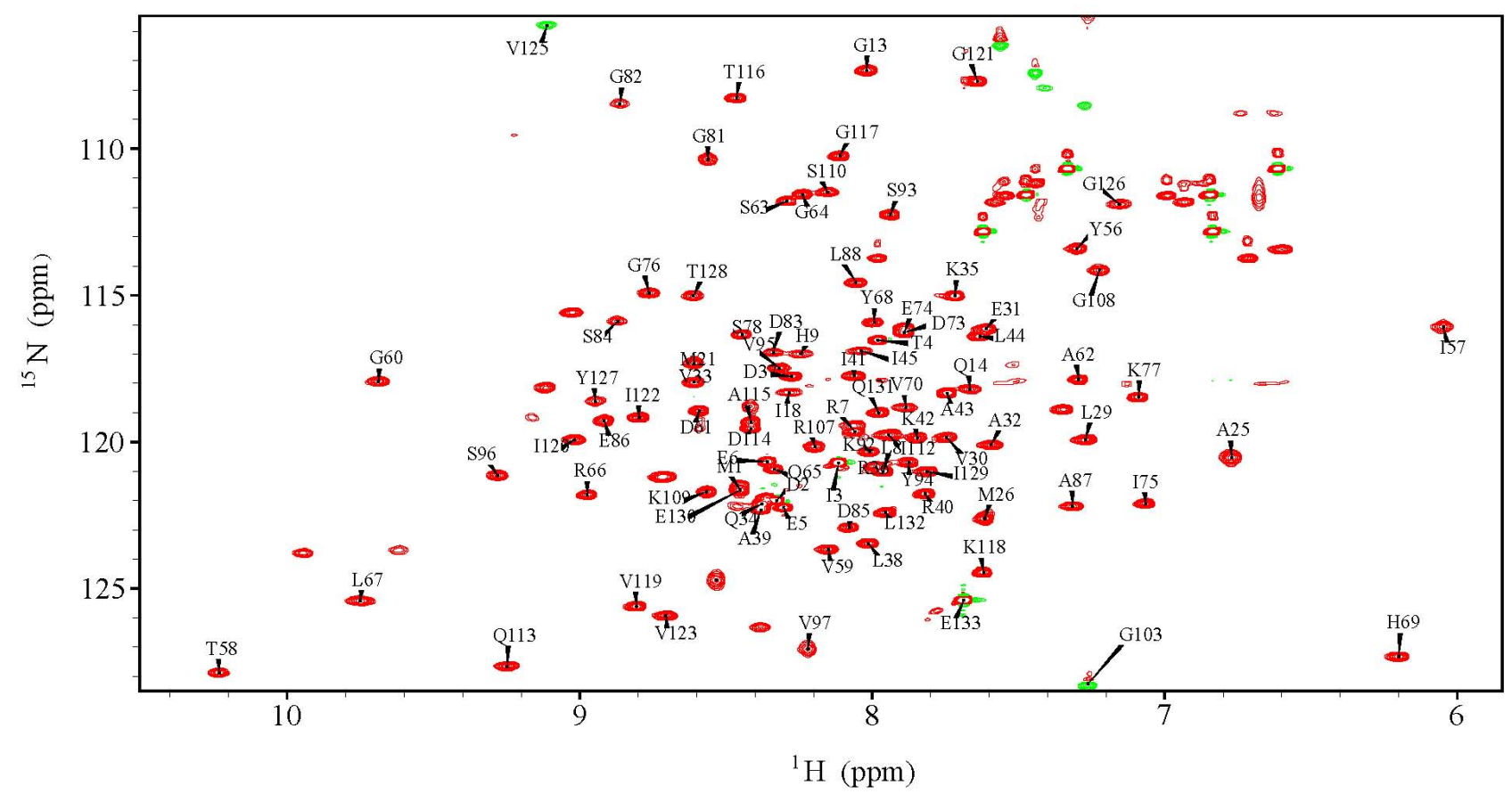

Figure 4.21: ${ }^{15} \mathrm{~N}-{ }^{1} \mathrm{H}$ HSQC spectrum of citrate bound CitAP with the assignment of amino acid given on top of the peaks. Ten fold excess of citrate was present in the solution. The spectrum was measured at $900 \mathrm{MHz}$ at $310 \mathrm{~K}$.

\subsubsection{X-ray structure of citrate bound form of CitAP without molybdate}

Due to lack of complete backbone assignments of the citrate free and bound form of CitAP, the NMR solution structure could not be determined. X-ray crystal trials were simultaneously performed using the protein made with the shorter construct and without histidine tag as well as without molybdate in the crystal screening buffer. Crystallization trials were carried out by Dr. Stefan Becker in our department. He obtained crystals of CitAP in the citrate bound form and solved the structure in collaboration with the group of Prof. George M. Sheldrick at the university of Göttingen. 
A

$\mathrm{B}$

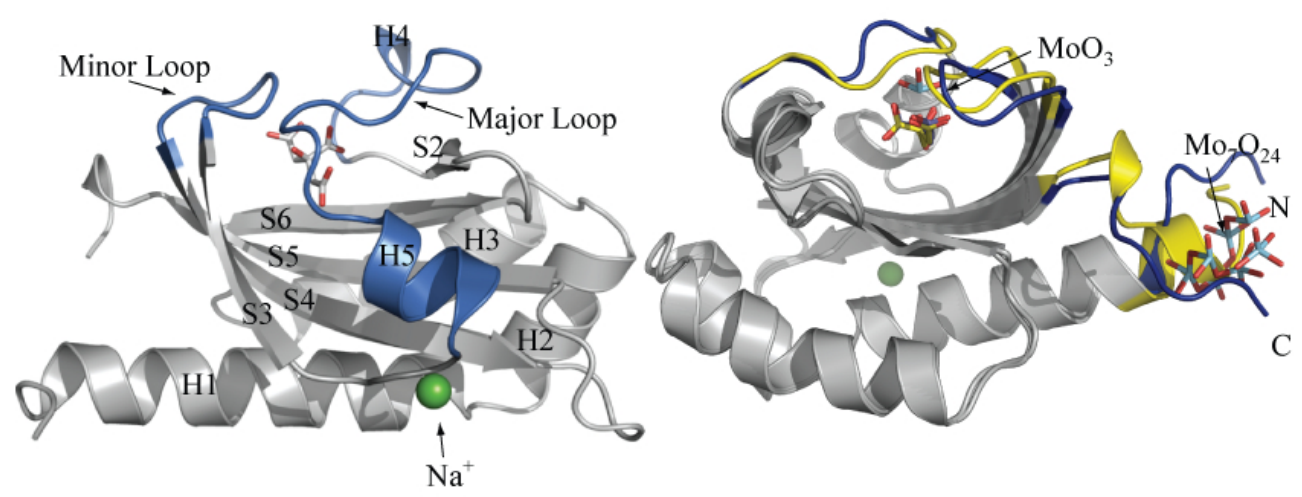

Figure 4.22: Citrate-bound structures of CitAP. (A) The secondary structural elements, major loop and minor loop residues highlighted in blue, bound $\mathrm{Na}^{+}$in green and the bound citrate in stick representation. (B) Superposition of 1P0Z (differences shown in dark blue) on the new citratebound structure (differences shown in yellow). Citrate is shown in stick representation (yellow: new structure, blue: $1 \mathrm{P} 0 \mathrm{Z}) . \mathrm{MoO}_{3}$ near the active site and bound $\mathrm{Mo}_{7} \mathrm{O}_{24}$ near the $\mathrm{N}$ - and C-terminus of $1 \mathrm{P} 0 \mathrm{Z}$ are are shown in stick representation.

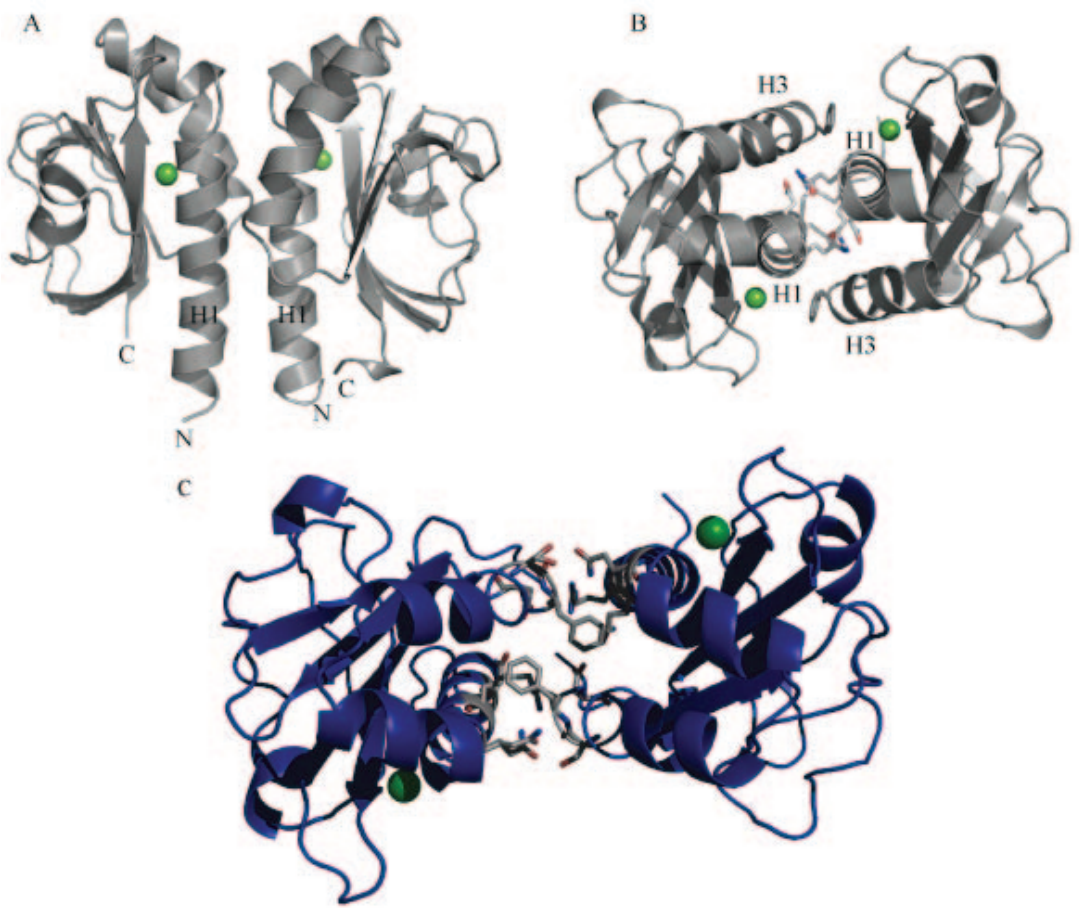

Figure 4.23: (A) Dimer in the asymmetric unit of our citratebound structure showing bound $\mathrm{Na}^{+}$ (green spheres). (B) Top view of the dimer in the asymmetric unit of our citrate-bound structure showing the interface residues. 
The crystallization condition was 0.1 M HEPES, pH 7.5, $1.6 \mathrm{M}\left(\mathrm{NH}_{4}\right)_{2} \mathrm{SO}_{4}$ and $5 \mathrm{mM}$ sodium citrate.

\section{Structure discussion}

The amino acid numbers used in the discussion of the X-ray and NMR part is the same as in the published CitAP structure. These residue numbers have an offset of 46 from the amino acid number in the CitA protein sequence.

There are two monomers in the asymmetric unit (citrate-bound-A: residues 4-132, citratebound-B: residues 6-129), which form a dimer. Structurally (Figure 4.22 panel A) each monomer has a PAS domain fold, highly similar to the monomers found in the asymmetric unit of 1P0Z (Figure 4.22 panel B) with an r.m.s.d. of only $1.2 \AA$. The bound citrate and the $\mathrm{Na}^{+}$in both structures superimpose nearly perfectly and their binding modes are very similar (Figure 4.22 panel B). The binding pocket is made up of the minor and major loop at the top and the $\beta$ sheet in the bottom. In this structure the backbone of the major loop (residues 63-92) is twisted between residues 78 and 84 by about 20 degrees in direction of the $\beta$-sheet, occupying the space where $\mathrm{MoO}_{3}$ is located in $1 \mathrm{P} 0 \mathrm{Z}$. Additionally the minor loop (residues 96-106) is relocated by about $1 \AA$ towards the major loop in our structure. Overall these conformational differences in minor and major loop result in a much tighter closure of the citrate binding pocket in this structure compared to 1P0Z. The extended conformations of the $\mathrm{N}$ - and C-termini in 1P0Z are likely to reflect both domain truncation and tight packing against the $\mathrm{Mo}_{7} \mathrm{O}_{24}$ cluster (Figure 4.17). In the new structure the N-terminal region has a helical conformation from residue 7 , and in the C-terminal region there is a 310 helix from residues 129-131 (monomer A). Several lattice contacts are made by the N-terminal residues. Therefore the specific structure of this region must also be considered with caution. NMR chemical shifts, on the other hand, support a significant helical propensity of residues 129131 in solution (Figure 4.36). In either case, the conformation of both termini may also be further altered by attachment to the trans membrane domains TM1 and TM2. 


\section{Dimer interface}
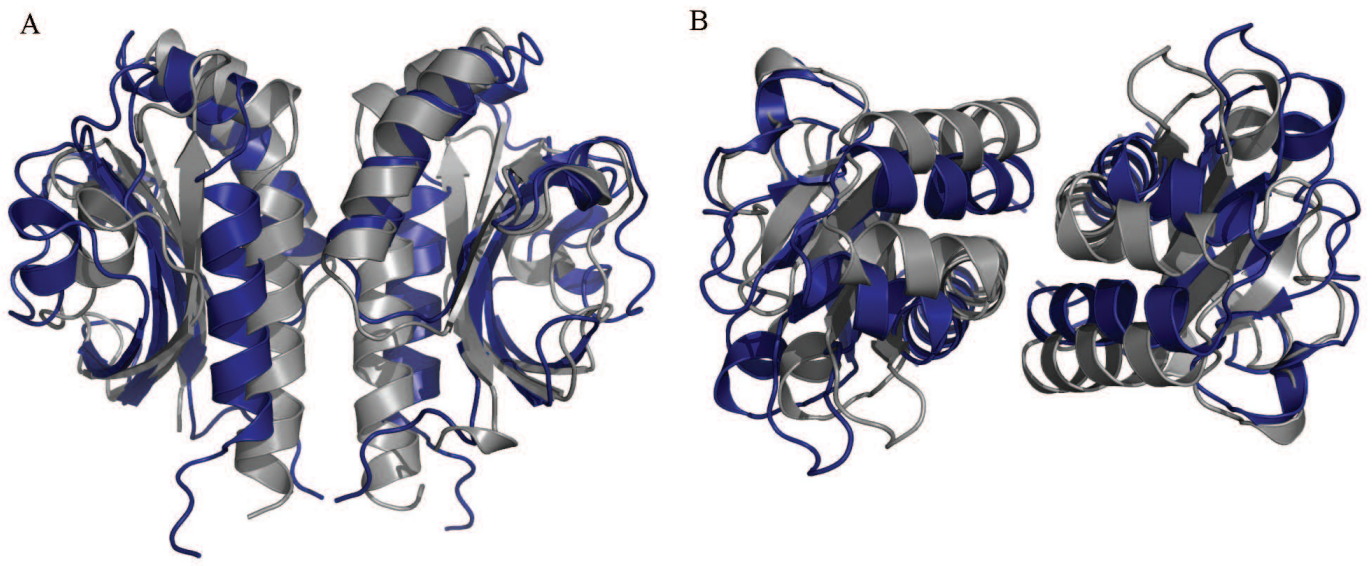

Figure 4.24: Superposition of the GJ type dimer of 1P0Z (dark blue) and the dimer of the new citrate-bound CitAP structure in the asymmetric unit. (A) side view (B) top view.

The overall shape of the dimer found in the asymmetric unit of the new citrate-bound CitAP structure (Figure 4.23 panel A) resembles the GJ-type dimer of 1P0Z (Figure 4.17). In $1 \mathrm{P} 0 \mathrm{Z}$ the dominant feature of this dimer interface is the pairwise parallel association of the N-terminal helix H1 from one monomer with the H3 helix of the other (Figure 4.23 panel C). In the new structure, helices $\mathrm{H} 1$ form a central parallel bundle involving residues 11-27, with helices H3 (residues 48-51) packed against them on either side (residues 21-25) (Figure 4.23 panel A and B). In the new structure dimerization mode results in a tightly packed dimer with an interface covering $2123 \AA^{2}$ of accessible surface area. The GJ-type dimer in 1P0Z is by far less tightly packed, with an interface of only $1415 \AA^{2}$. The superposition of their backbones (Figure 4.24 results in an r.m.s.d. of $3.6 \AA$, compared to only $1.2 \AA$ for the monomers, emphasizing the distinct dimer packing interfaces. Both structures differ also strongly in the hydrophobicity of their interface. In the GJ-type dimer of 1P0Z the Phe51 residues form a central hydrophobic patch (Figure 4.23 panel $\mathrm{C}$ ) that is extended via hydrophobic interactions into the hydrophobic cores of both monomers. In the new structure the interface between the monomers is in the region between helices H1 by far less hydrophobic. Thus, despite high structural similarity between the monomers, the dimerization modes in the new citrate-bound CitAP structure and in the GJ-type dimer from 1P0Z are very different in 
size and nature. Given the more extensive interface observed, the new citrate-bound dimer is more likely to represent the physiological association.

\subsubsection{X-ray structure of citrate free form of CitA}

Structure of citrate free CitAP was yet to be determined. Hence simultaneously with the crystallization of the citrate bound form of CitAP, Xray crystal trials were done on the free form as well. The citrate free CitAP was able to be crystallized in a buffer containing 20 mM HEPES, pH 7.5, $0.63 \mathrm{M} \mathrm{NaH}_{2} \mathrm{PO}_{4}$ and $0.63 \mathrm{M} \mathrm{KH}_{2} \mathrm{PO}_{4}$. The structure of citrate-free CitAP was determined to a resolution of $2.0 \AA$. There are two monomers in the asymmetric unit (citrate-free-A and citrate-free-B) (Figure 4.25). In citrate-free-A residues 1 to 131 were traced, except for a part of the major loop (76-85). In citrate-free-B, residues 3 to 128, including the complete main chain of the major loop residues (63-92), were traced (yellow in Figure 4.25). For a number of these residues the refinement resulted in decreased occupancies. This suggests mobility of the major loop, in agreement with the fact that part of the major loop residues could only be traced in one of the monomers. Pairwise r.m.s.d of backbone residues of the two monomer structures show large deviations in the major loop region (Figure 4.35, top panel). Only 12 common hydrogen bonds were found between the two monomer structures. This is a hint toward a very flexible structure for the citrate free form of CitAP.

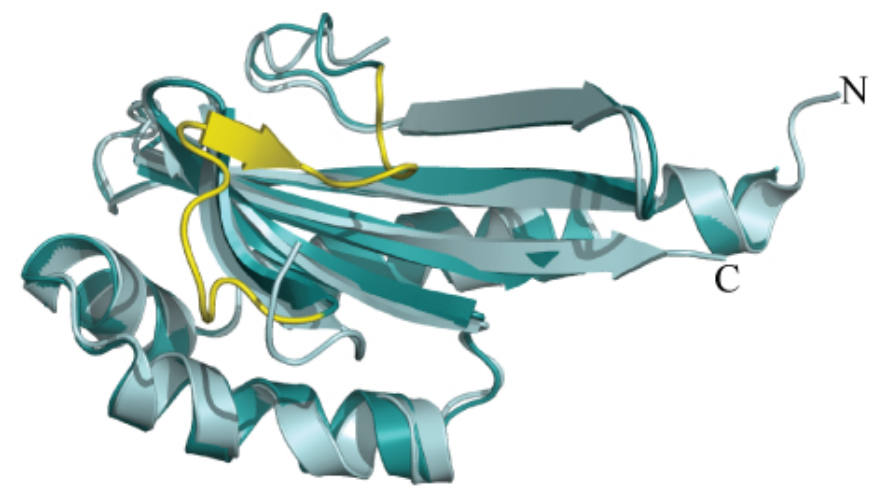

Figure 4.25: Structure of Citrate-free CitAP with superposition of both monomers in the asymmetric unit. The major loop that could be traced in one of the monomers is colored in yellow 


\subsubsection{NMR studies on the citrate free form of CitAP}

Only $62 \%$ of the possible peaks were observed in the ${ }^{15} \mathrm{~N}^{-1} \mathrm{H}$ HSQC spectrum of the citrate free form of CitAP. The average rotational correlation time $\left(\tau_{c}\right)$ measured for the protein was $7.54 \mathrm{~ns}$, indicating a monomeric CitAP in solution. No improvement and no additional peaks were observed with TROSY-HSQC spectrum. These results suggest that line broadening due to conformational exchange might be the cause of the disappearence of around $40 \%$ of the peaks.

Usually, three dimensional experiments for protein backbone assignments are acquired with amide proton detection in the direct dimension. These amide protons are the most sensitive to line broadening due to chemical exchange. In order to limit line broadening due to conformational exchange, several non-standard NMR experiments were acquired. To reduce amide proton chemical exchange broadening, CPMG-HSQC experiments were measured. CPMG sequence was applied during the INEPT (refer to Chapter 1 for description of INEPT) transfer period in the normal HSQC. Relaxation due to chemical exchange is reduced in the CPMG sequence. No additional peaks were obtained in the CPMG-HSQC spectra.

Rather than using amide detection in the direct dimension, $\mathrm{H}_{\alpha}$ detected experiments were also tried. Back and forth $\mathrm{HACACO}$ experiment with $\mathrm{H}_{\alpha}$ detection in the direct dimension did not give any additional peaks (refer to Chapter 2, section 2.2 for more details). Only 80 peaks were observed in the spectrum (Figure 4.26 panel $\mathbf{A}$ ). This implies that line broadening due to chemical exchange is not restricted to amide group only.

To determine the presence of conformational exchange in the side chain resonances in the protein, ${ }^{13} \mathrm{C}-{ }^{1} \mathrm{H}$ HSQC experiment was measured. Methyl groups have the most favorable $\mathrm{T}_{2}$ relaxation time due to rapid rotation of its carbon-carbon single bonds. The ${ }^{13} \mathrm{C}-{ }^{1} \mathrm{H}$ HSQC spectrum of the methyl region of the citrate free CitAP shows only 62 peaks (Figure 4.26 panel B ). 85 possible peaks were expected from the protein sequence. The missing number of methyl resonances fit well with the number of methyl peaks that were expected from the unassigned amino acids in the protein sequence. This proves that line broadening due to 

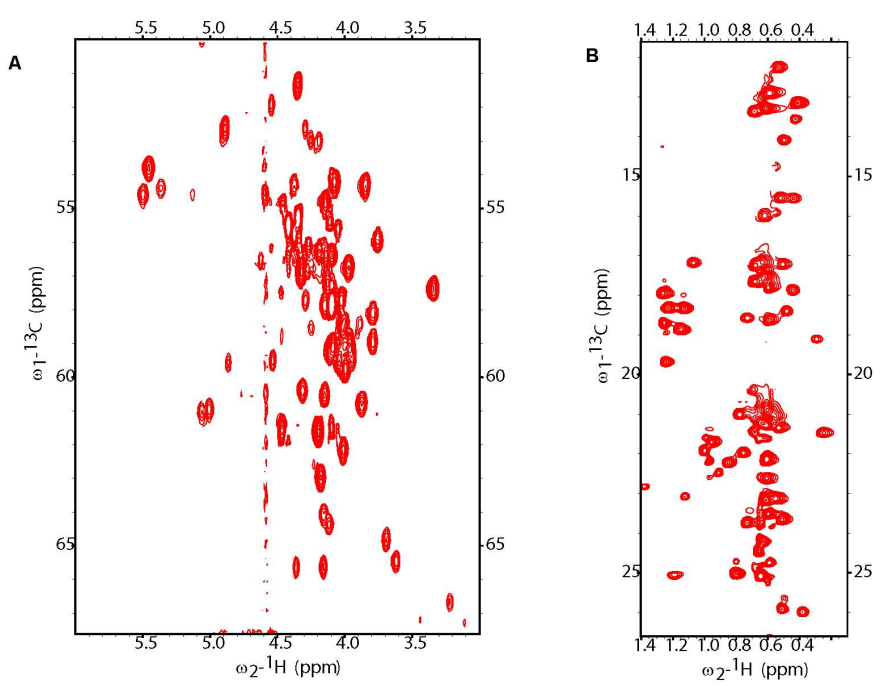

Figure 4.26: (A) ${ }^{13} C_{\alpha^{-}}{ }^{1} \mathrm{H}_{\alpha}$ projection of three dimensional HACACO experiment. Only 80 peaks are observed in this spectrum. (B) ${ }^{13} \mathrm{C}^{-1} \mathrm{H}$ HSQC spectrum of the methyl region. Only 62 peaks out of 85 peaks are seen in the spectrum.

conformational interchange affect the side chain resonances as well. These results indicate that there must be different conformations of CitAP in solution. This is in accordance with the identification of two different structures for the monomers of CitAP in the asymmetric unit of the citrate free CitAP crystals.

Nevertheless, the backbone assignment of 72 of the 80 peaks observed in the HSQC spectrum were possible. The unassigned residues of CitAP is plotted onto the monomer B of the structure of citrate free form of CitAP (Figure 4.39 ). It is interesting to observe that most of the unassigned residues in the sequence are those residues which show large backbone r.m.s.d deviations between the two forms of the monomers of the citrate free structure of CitAP. These residues are mostly in the two $\beta$ sheets and the parts of the major loop which also defines the binding pocket for citrate in the citrate bound structure. The three residues that are assigned in the major loop region have considerably high flexibility associated with 
it (refer to discussion below), this could have reduced the relaxation process which leads to the disappearance of the other peaks in the major loop.

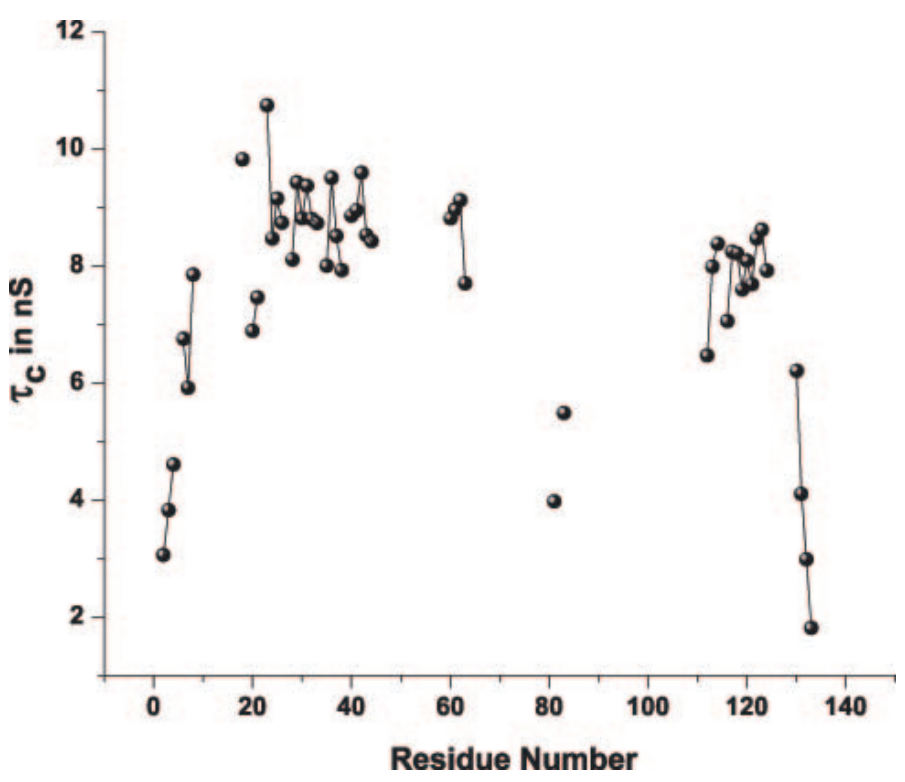

Figure 4.27: Plot of measured rotational correlation time against individual residue number of citrate free-CitAP. The plot gives a measure of flexibility of residues. Residues at the $\mathrm{N}$ and $\mathrm{C}$ terminal and the two residues that was assigned in the major loop region show low $\tau_{c}$ values indicating major flexibility in these region.

Usual $\mathrm{T}_{1}, \mathrm{~T}_{2}$ methods for estimation of $\tau_{c}$ have $R_{e x}$ (relaxation rate due to conformational exchange) term associated with it. For proteins for which $R_{e x}$ is the dominant relaxation rate, these methods give a very large value for $\tau_{c}$. Hence cross correlated relaxation rate was used to determine the $\tau_{c}$ values. The $\tau_{c}$ values obtained using this method does not have contribution from chemical exchange. As indicated earlier, the average $\tau_{c}$ of CitAP determined using this method (described in 2.2.8) is 7.54 ns. Spectral density function of a rigid body is used in the calculation for $\tau_{c} \mathrm{~s}$. Hence a lower bound for the values of $\tau_{c} \mathrm{~S}$ are obtained using this method. $\tau_{c}$ for individual amino acids are determined. As described in chapter 2, section 2.2.8, the $\tau_{c}$ values are a function of the amplitude of local motion associated with that amino acid residue $\left(S^{2}\right)$. Residues with lower $\tau_{c}$ values than the average value have significant flexibility term associated with it. Most of the residues showing low $\tau_{c}$ values are in the $\mathrm{N}$ and $\mathrm{C}$ terminals. The $\tau_{c}$ for the amino acids assigned in the major loop 
region also have lower than average value, indicating high flexibility and deviation from rigid body approximation (Figure 4.27). Heteronuclear NOE experiment was also measured for the citrate free CitAP. The amplitude of motions in the pico- to nano-second time-scale is investigated with the steady state ${ }^{15} \mathrm{~N}-{ }^{1} \mathrm{H}$ NOE. ${ }^{15} \mathrm{~N}-{ }^{1} \mathrm{H}$ Het-NOE values therefore give a measure of residue flexibility. The three residues for which assignment was possible in the major loop (residues 81,83 and 84), have lower Het-NOE value (lower than 0.6) indicating high flexibility in this region (Figure 4.28). This correlates well with the lower $\tau_{c}$ values of these residues (residues 81 and 83 ).

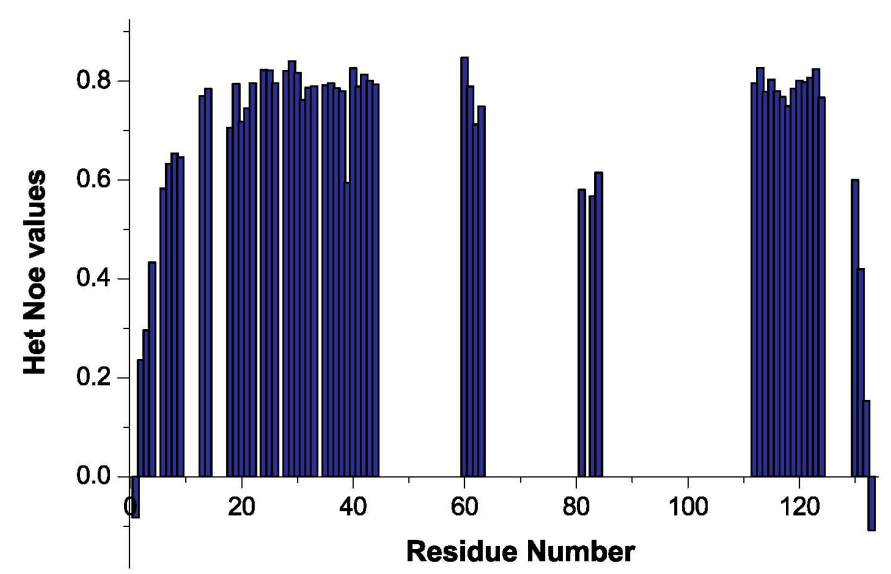

Figure 4.28: ${ }^{15} \mathrm{~N}-{ }^{1} \mathrm{H}$ NOE values measured for citrate free-CitAP are plotted against residue number. Residues near the $\mathrm{N}$ and $\mathrm{C}$ terminal and the residue in the main loop region have lower Het-NOE values indicating high flexibility.

\subsubsection{NMR studies on the citrate bound form of CitAP}

Citrate bound form of CitAP was obtained by the addition of one equivalent of citrate to the protein sample. Binding of citrate to CitAP is in the slow exchange limit. 107 peaks were observed in the ${ }^{15} \mathrm{~N}-{ }^{1} \mathrm{H}$ HSQC spectrum of the citrate bound form of CitAP. Backbone assignment was obtained for hundred of these peaks. The unassigned residues are mainly located in the N-terminal helix (H1: residues 9-12, 15-19, and 22-24), linker between the H3 helix and the first $\beta$ strand (residues 48-54) and in the citrate binding area (mainly in the minor loop, residues 97-102) (refer Figure 4.30). These residues might be broadened beyond detection because of chemical exchange. As discussed for the crystal structure of citrate 


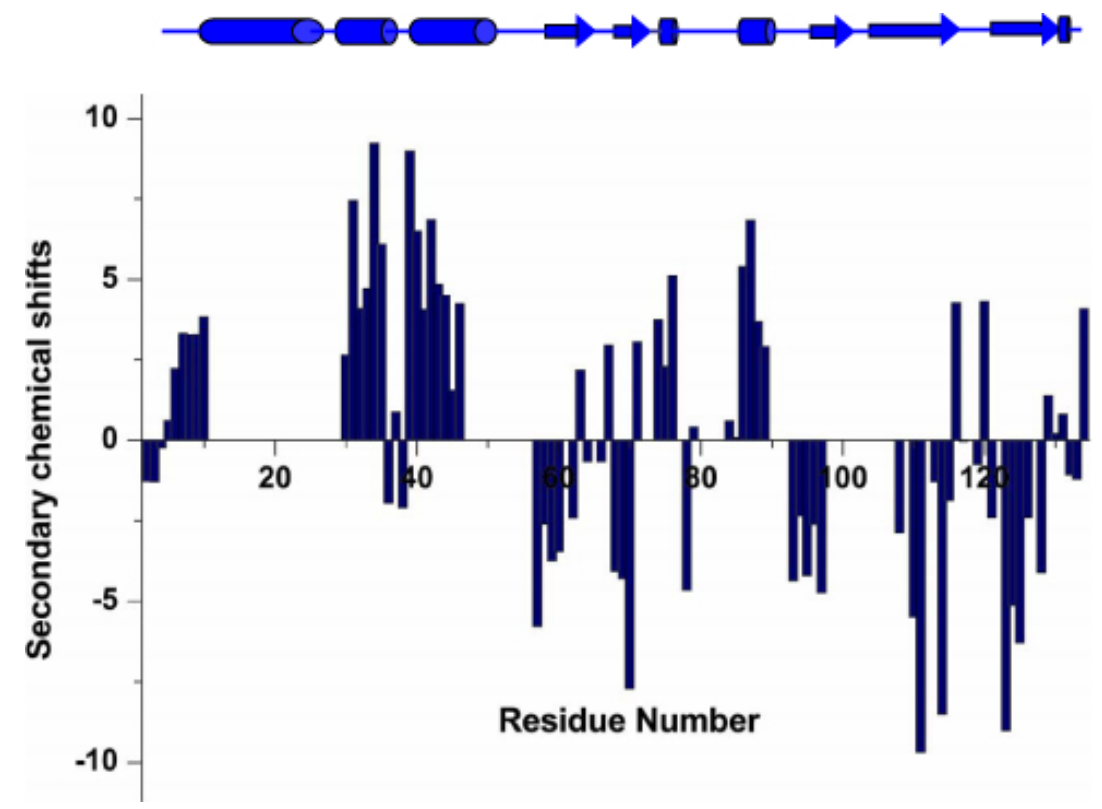

Figure 4.29: Secondary structure of citrate bound CitAP. Secondary chemical shifts of $\Delta \mathrm{C}_{\alpha^{-}}$ $\Delta \mathrm{C}_{\beta}+\Delta \mathrm{C}$ '.Secondary structural element from the new citrate bound crystal structure of CitAP is given at the top of the figure.

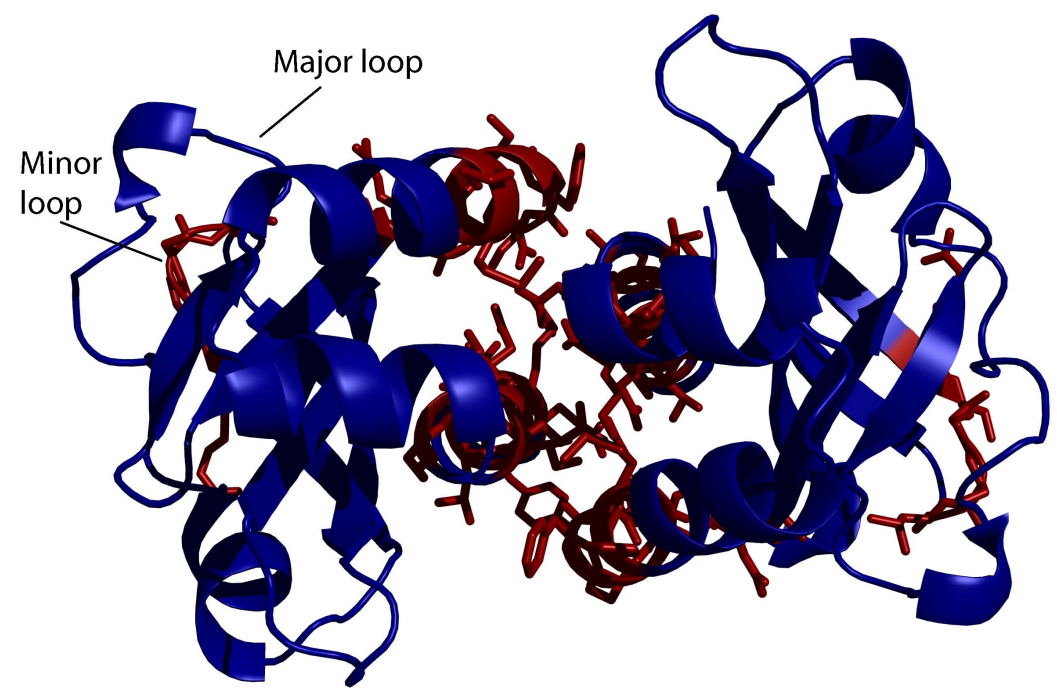

Figure 4.30: The unassigned residues in citrate bound form of CitAP are plotted on to the structure of citrate bound dimer. The unassigned residues (red sticks) are mainly located in the dimer interface and also in the minor loop of CitAP. 
bound CitAP, the $\mathrm{N}$ terminal helix (namely Helix1) is involved in the dimer interface. This might be the reason for the chemical exchange broadening of the peaks due to a possible dimer-monomer exchange (residues in the H1 and H3 helix are involved in dimer interface), though previous studies indicate the presence of only $10 \%$ dimer in the solution of citrate bound form of CitAP.

The secondary structure element was determined from the deviation of the $\mathrm{C}_{\alpha}, \mathrm{C}_{\beta}$ and C' chemical shifts from the random coil values and are shown in Figure 4.29. Positive values indicate helical propensity and negative value denote $\beta$ strand propensity. The plot of the secondary chemical shifts of citrate bound CitAP correlate well with the secondary structural elements from X-ray structure of the citrate bound form of CitAP (Figure 4.29, top panel). ${ }^{15} \mathrm{~N}-{ }^{1} \mathrm{H}$ HetNOE values were also measured. Heteronuclear amide NOE values larger than 0.7 for most of the residues of bound form of CitAP imply rigidity of the structure. Amide $\mathrm{NOE}$ values are slightly decreased in the $\mathrm{N}$ and $\mathrm{C}$ terminal regions of the protein indicating flexibility in these regions.

\subsubsection{Sodium binding to CitAP}

Early microbiological evidence had indicated that anaerobic growth of K.pneumoniae on citrate was $\mathrm{Na}^{+}$dependent [147, 148, 149]. It was also previously shown that induction of the target genes by CitA/CitB requires not only citrate, but also $\mathrm{Na}^{+}$[138]. This feature is physiologically meaningful as both citrate transport and metabolism are also strictly dependent on $\mathrm{Na}^{+}$[150, 151]. The X-ray structures seem to suggest a functional connection between citrate and $\mathrm{Na}^{+}$binding to CitAP, as $\mathrm{Na}^{+}$was identified only in the citrate-bound CitAP structure. Although valence bond calculations strongly support this choice [152], the identity of the $\mathrm{Na}^{+}$modeled in this structure is not absolutely certain. Similarly, the fact that we could not identify an ordered $\mathrm{Na}^{+}$in the citrate-free form does not strictly exclude the possibility that $\mathrm{Na}^{+}$may be present but disordered. However NMR studies corroborate the $\mathrm{Na}^{+}$binding to the citrate bound form of CitAP. 
Significant chemical shift changes and sharpening of peaks are the characteristic changes observed in the ${ }^{15} \mathrm{~N}-{ }^{1} \mathrm{H}$ HSQC when citrate is bound to CitAP. These changes are observed with a one fold addition of sodium citrate to CitAP. On further addition of sodium citrate (10 fold to 20 fold) in excess, small chemical shift changes were observed (Figure 4.31 left). Specific subset of residues are affected in the titration. Those residues which show additional changes are in a well defined region in the CitAP structure, which corresponds to the $\mathrm{Na}^{+}$ binding site in the CitAP structure (Figure 4.31 right). Hence as evident from the $\mathrm{Na}^{+}$ titration, citrate bound form of CitAP binds sodium in the fast exchange time scale.
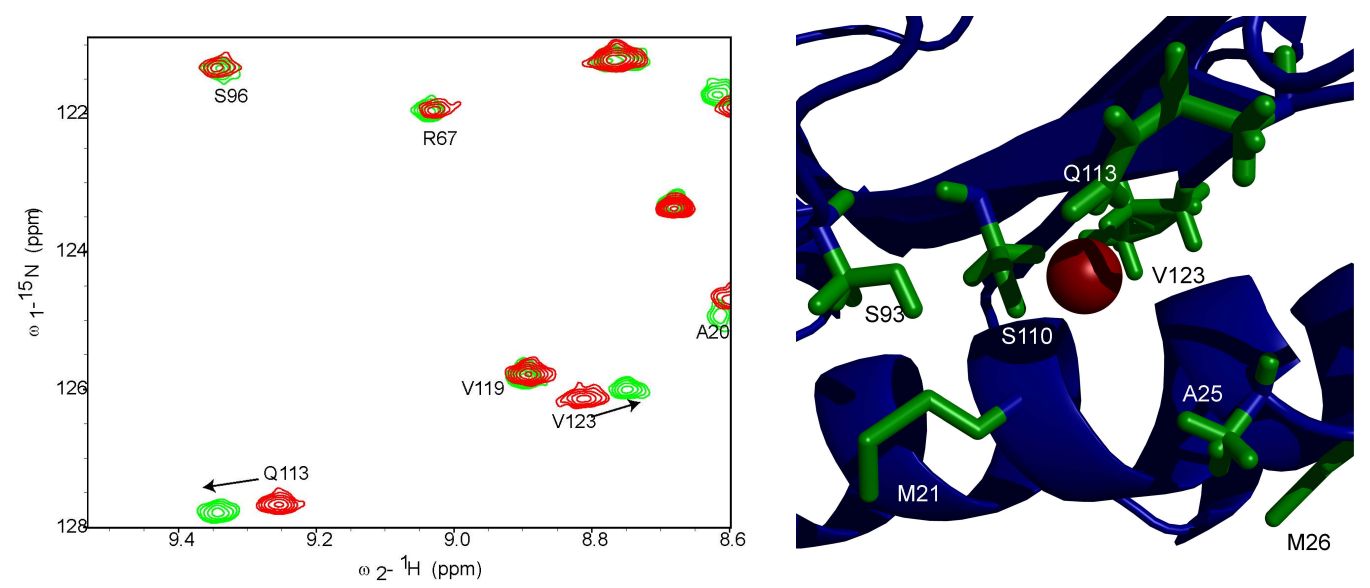

Figure 4.31: (left) Effect of $\mathrm{Na}^{+}$on citrate bound CitAP. Overlay of the portion of HSQC showing few peaks affected by $\mathrm{Na}^{+}$titration. Peaks in red are from spectrum measured for one equivalent of sodium citrate in the protein sample and peaks in green are measured at 20 fold excess of sodium citrate. (right) Citrate bound-CitAP structure: withr residues most affected by $\mathrm{Na}^{+}$titration in stick representation. $\mathrm{Na}^{+}$ion is given in red sphere. The residue shown are M21, A25, M26, S93, S110, Q113 and V123.

\subsubsection{Residual dipolar coupling analysis}

Residual dipolar couplings (RDCs) analysis is an efficient method to determine whether the X-ray structure of a protein is retained in solution (refer to chapter 1, section 1.3). To check for the presence of the X-ray structure of CitAP in solution, residual dipolar couplings were measured. The citrate free and bound form of CitAP were aligned in bacterial filamentous phages. Quadrupolar deuterium splitting was 9 and $7 \mathrm{~Hz}$ respectively. ${ }^{1} \mathrm{D}_{N H}$ RDCs were measured for citrate bound CitAP while a set of ${ }^{1} \mathrm{D}_{N H},{ }^{1} \mathrm{D}_{N C}$ and ${ }^{1} \mathrm{D}_{C C \alpha} \mathrm{RDCs}$ were measured for citrate free CitAP. 

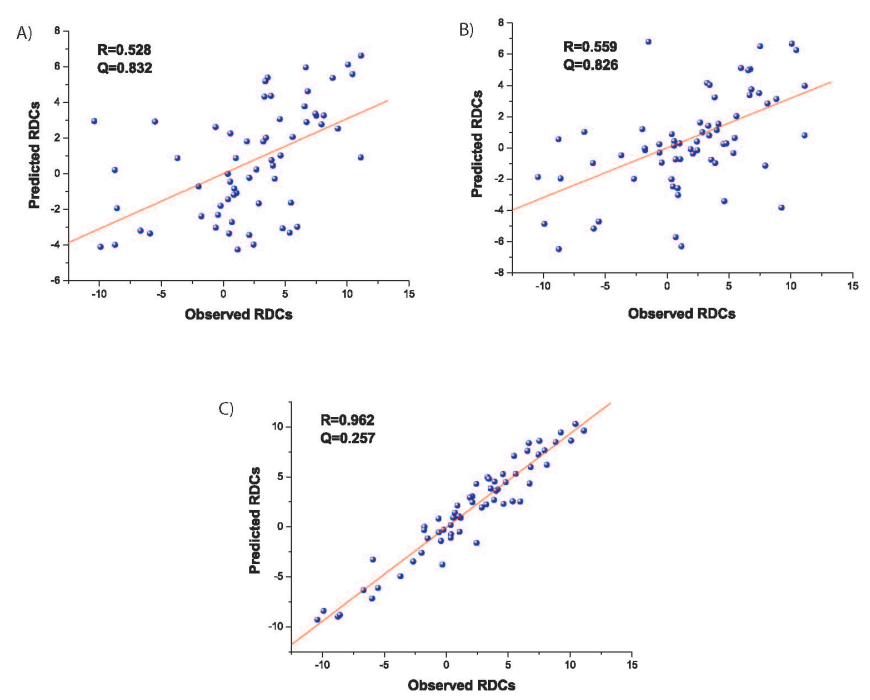

Figure 4.32: Correlation of measured H-N RDCs of citrate bound CitAP with back calculated RDCs from different crystal structures of CitAP. (A) Correlation between measured HN RDCs with back calculated RDCs from monomer A ( (A)), monomer B ( (B)) of citrate free-CitAP and the new citrate bound structure of CitAP ( (C)). The correlation coefficients were $0.528,0.559$ and 0.962 respectively.

\section{RDCs of citrate bound CitAP}

PALES software was used to fit the measured residual dipolar couplings to the structures using the SVD (Single Value Decomposition) method. 75 well resolved NH couplings were obtained for the citrate bound form of CitAP. Measured RDCs gave a correlation of 0.962 with the new citrate bound structure of CitAP (refer Figure 4.32 panel C). As expected the same RDCs gave a very bad correlation with the two free structures of CitAP (refer Figure 4.32 panel A and B). Good correlation of the measured citrate bound RDCs with the bound structure suggest that in solution structure of the bound form of CitAP is very similar to crystal structure of the bound form. Bad correlation of the bound RDCs with the citrate free structures indicate that there are hardly any contribution from the free structures to the bound RDCs and that the citrate bound structure would be very different from the citrate free structure. 


\section{RDCs of citrate free CitAP}

66 well resolved $\mathrm{N}-\mathrm{H}$ and $\mathrm{N}-\mathrm{C}$ ' couplings were obtained for the citrate free form of CitAP. RDCs of the free form of CitAP fit with a correlation of 0.825 with the monomer A of the crystal structure of citrate free CitAP (Figure 4.33 panel A). The measured RDCs also fit to 0.763 with monomer B of the citrate free form of CitAP (Figure 4.33 panel B). Surprisingly the same RDCs gave a correlation of 0.93 with the new citrate bound structure of CitAP (monomer A). (Figure 4.33 panel C). It is possible that the measured RDCs could have contribution from both the monomer structures in the citrate free form. In order to analyze this, simultaneous fit of the measured RDCs with the two monomer structures of CitAP were carried out. A non linear fit implemented in MATHEMATICA using a single alignment tensor for both the monomer structure of the citrate free CitAP yielded a correlation of 0.836 (Figure 4.33 panel D). The fitting procedure is explained in more detail in Chapter 2, section 2.2.5. These results can be explained on the basis of multiple conformation of protein in solution. The measured RDCs will have contributions from all of the conformers of the protein in solution. Only slight increase in the correlation was observed with the simultaneous fit of the RDCs with the two citrate free monomer structures of CitAP. This suggests that there may be more possible conformers in solution. It could also be that the X-ray structures of citrate free CitAP are not well defined and the increase in the correlation with the simultaneous fit could just be the averaging of structural noise in these two structures. Hence these results have to be taken with caution. Nevertheless taking these results and severe line broadening observed for the peaks in the ${ }^{15} \mathrm{~N}-{ }^{1} \mathrm{H}$ HSQC it is reasonable to assume the presence of multiple conformers of CitAP in solution. The correlation of measured RDCs with those derived from monomer A of citrate free CitAP and the new bound structure of CitAP was 0.936 (Figure 4.33 panel E). The fitted weighting factors were 0.18 for the monomer $\mathrm{B}$ and 0.82 for the bound citrate-CitAP structure. Same correlation was obtained by using the two structures of the citrate free CitAP and the new citrate bound CitAP (Figure 4.33 panel G). The fit converged only when the weighting factor for monomer B of citrate free CitAP approached zero. This could be explained by a badly defined X-ray structure or an improbable conformer in solution. So the outcome of the RDC analysis is that in the citrate free solution, the major 
population of the protein is in the citrate bound coformer (about 80\%) and a part in the citrate free monomer A form (About 20\%). Better correlation of the measured RDCs with the citrate bound structure suggest that the bound structure is one of the conformer present in the citrate free CitAP solution giving a significant contribution to the measured RDCs of citrate free CitAP.

\subsubsection{Comparison of citrate free CitAP and citrate bound CitAP using X-ray and NMR}

The major difference in the structures of citrate free and citrate bound CitAP are in the $\mathrm{N}$ and $\mathrm{C}$ terminals and in the major and minor loops. In both citrate-free- $\mathrm{A}$ and citratefree-B the short helices H4 (71-74) and H5 (86-90), which are found in the major loop in the new citrate-bound CitAP structure, are unwound (Figure $4.34 \mathrm{~A}$ ). Instead, a short strand is formed by residues 78-81 in citrate-free-B. A superposition of citrate-free-B with citrate-bound-A (Figure $4.34 \mathrm{~B}$ ), shows that a part of the major loop fills the space in the citrate-free form that is occupied by citrate in the citrate-bound form. Although the overall structure of citrate-free CitAP is similar to the new citrate-bound structure, the central fivestranded anti-parallel $\beta$-sheet is less bent in the citrate-free state. As a result, the tip of the minor loop has moved by $13.51 \AA$ away from its position in citrate-bound CitAP (Figure 4.34 A). This wide open conformation of the minor loop (formed partly by strands S3 and S4) causes the strand S5 to extend up to the C-terminus. A typical $\beta$-sheet-type backbone hydrogen-bonding pattern exists between residues of the C-terminal region and residues of the neighboring strand S4 (green in Figure $4.34 \mathrm{~b}$ ). The helix H1 is slightly extended in the citrate free form of CitAP compared to bound form of CitAP. In the C-terminal part, a $3_{10}$ helix is found in the bound form of CitAP, while in the citrate free-CitAP, the strand S5 extend up to $\mathrm{C}$ terminus.

\section{NMR studies}

The back-bone structure of CitAP becomes more rigid with the binding of citrate to CitAP. This is indicated by the positive values obtained from the difference in Het-NOE values of citrate bound and citrate free form for most of the residues (Figure 4.36 middle panel). Major 

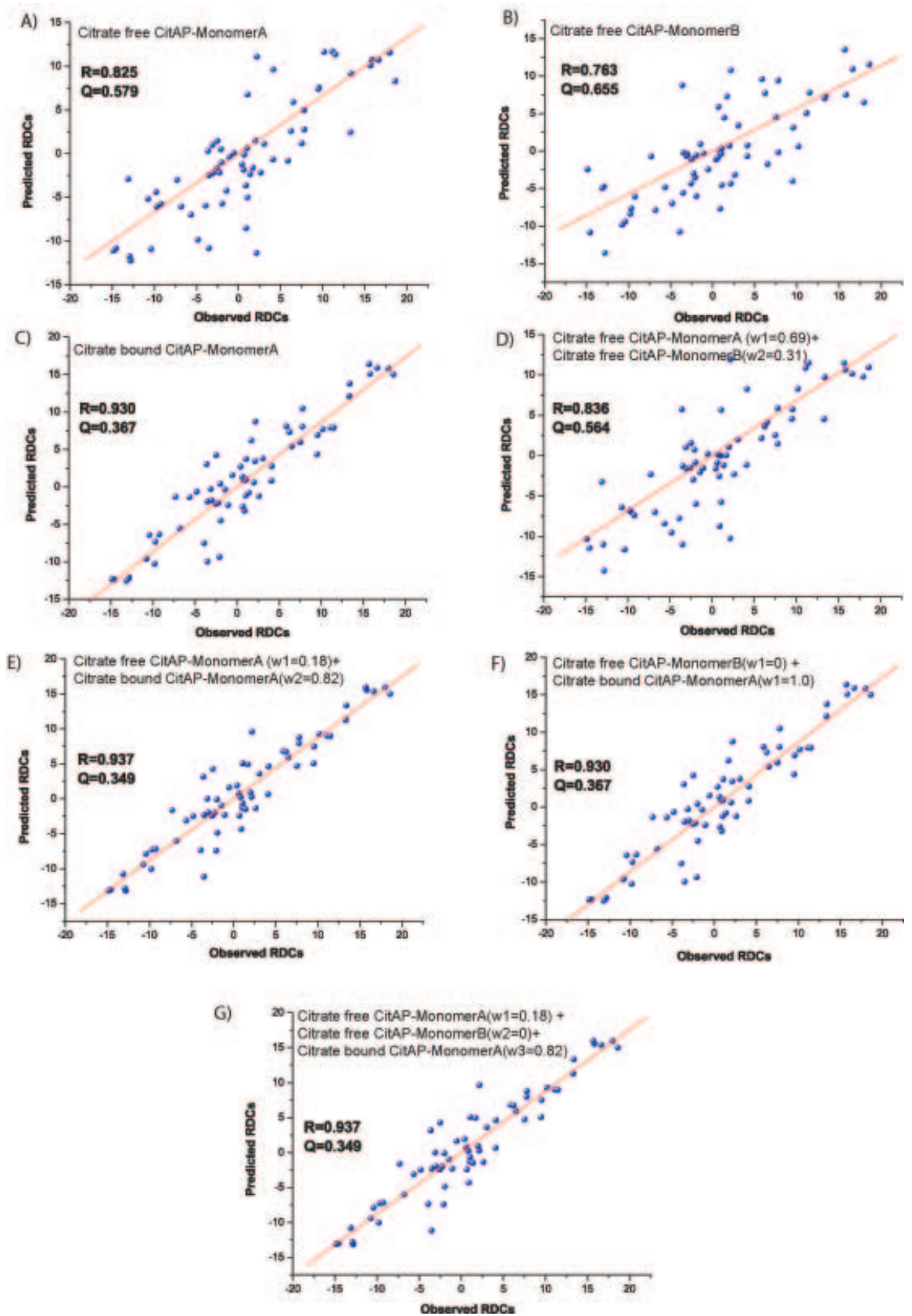

Figure 4.33: Correlation between measured RDCs of citrate free-CitAP with back calculated RDCs from different Xray structures of CitAP and their combinations. Correlation between the measured $\mathrm{N}-\mathrm{H}$ and N-C' RDCs with back calculated RDCs from single alignment tensors derived from X ray structures: monomer A of citrate free-CitAP $(\mathbf{A})$, monomer B of citrate free-CitAP $(\mathbf{B})$ and citrate bound CitAP structure $(\mathbf{C})$. Correlation of the measured RDCs with back calculated RDCs using a single alignment tensor and a population weighting factor derived by campaigning two or more $\mathrm{X}$ ray structures: monomer $\mathrm{A}$ and $\mathrm{B}$ of citrate free-CitAP $(\mathbf{D})$ : the population factors were 0.69 and 0.31 respectively, monomer A of citrate free CitAP and citrate bound CitAP (E): population factors were 0.18 for monomer A and 0.82 for citrate bound strcture, monomer B of citrate free CitAP and citrate bound CitAP $(\mathbf{F})$ : population factor was zero for monomer B and 1.00 for citrate bound structure, monomer A and B of citrate free CitAP and the citrate bound $\operatorname{CitAP}(\mathbf{G})$ : the poplulation factore was zero for monomer B of citrate-free CitAP and 0.18 for monomer A of citrate-free CitAP, the population factor was 0.82 for the citrate bound form. 


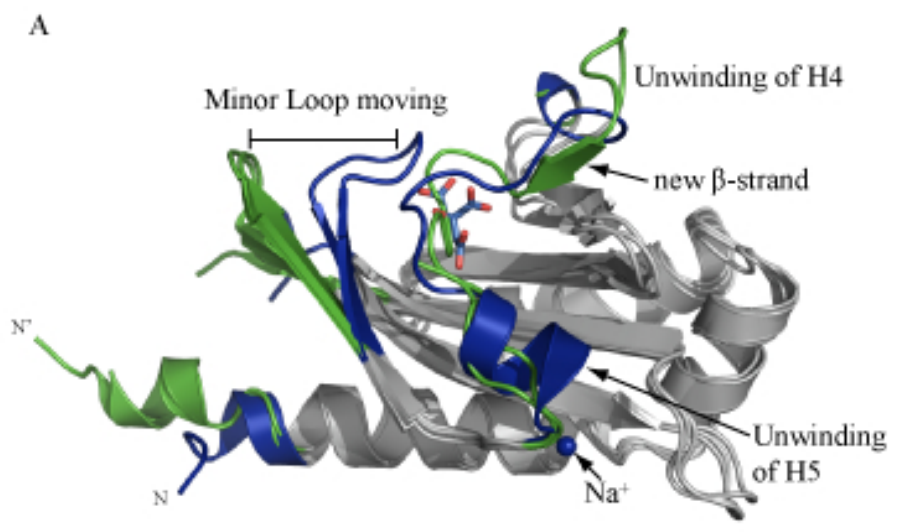

B

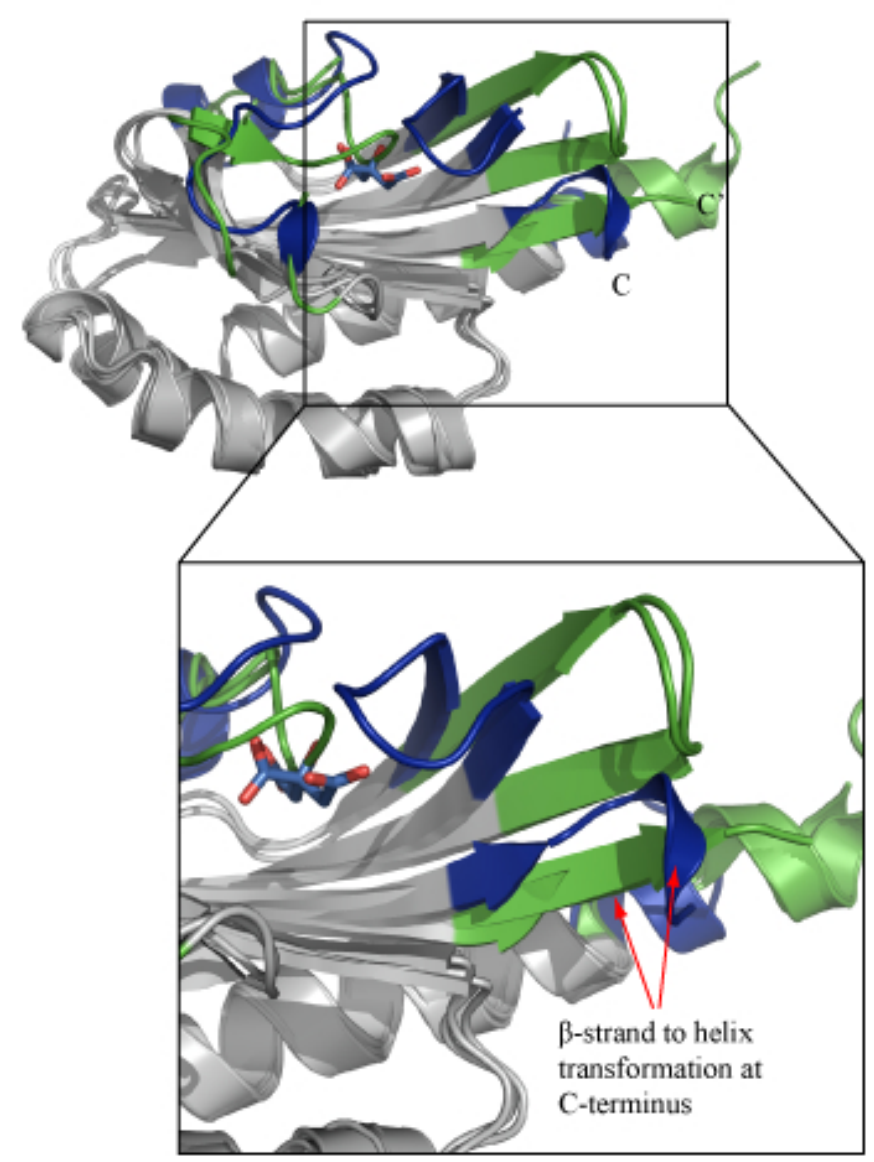

Figure 4.34: Conformational differences between citrate-bound and citrate-free CitAP. (A) Side view of superposition of citrate-bound structure (differences highlighted in dark blue) on citratefree structure (both monomers) (differences highlighted in green) showing the minor loop movement of $\sim 45^{\circ}$. (B) View from opposite side of the superposition of citrate-bound CitAP (differences highlighted in dark blue) on the citrate-free structure (both monomers) (differences highlighted in green) showing the displacement of the major loop upon citrate binding, the $\beta$-strand in the major loop of the citrate-free structure and the conformational difference in the C-terminal region (inset). The displacement of the C-terminus is indicated by the location of residue Gln131. 

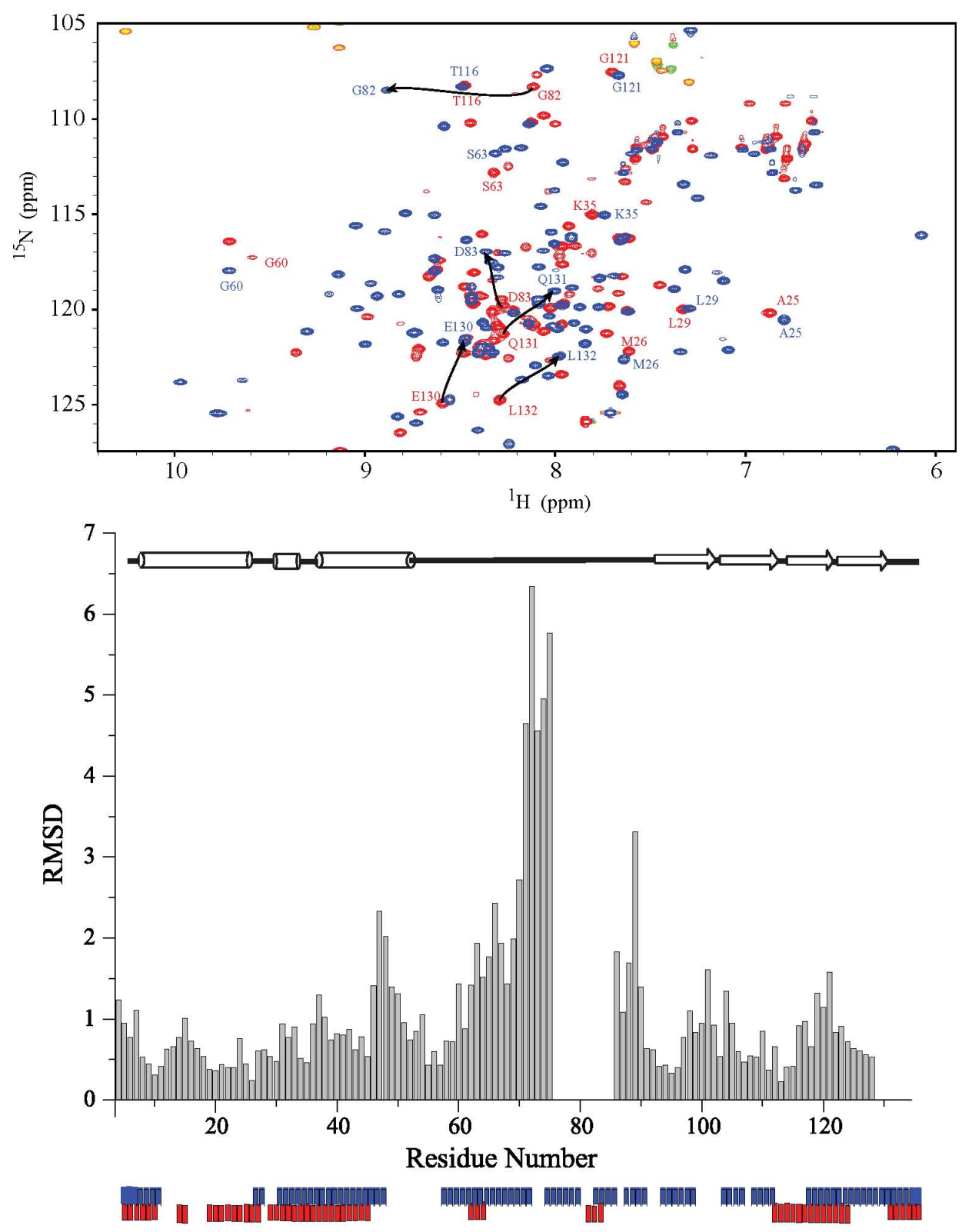

Figure 4.35: Top panel: Overlay of HSQC spectra showing the free (red) and citrate bound (blue) resonances for CitAP. Arrows follows those residues which show a large chemical shift change upon citrate binding. Bottom panel: Backbone r.m.s.d plot (grey) for the two free monomer structures of CitAP. Red and blue bars indicate respectively those residues of CitAP for which NMR resonance assignments were obtained for the citrate-free and citrate-bound form. Secondary structural elements from the citrate free structure is given on top of the r.m.s.d plot. 

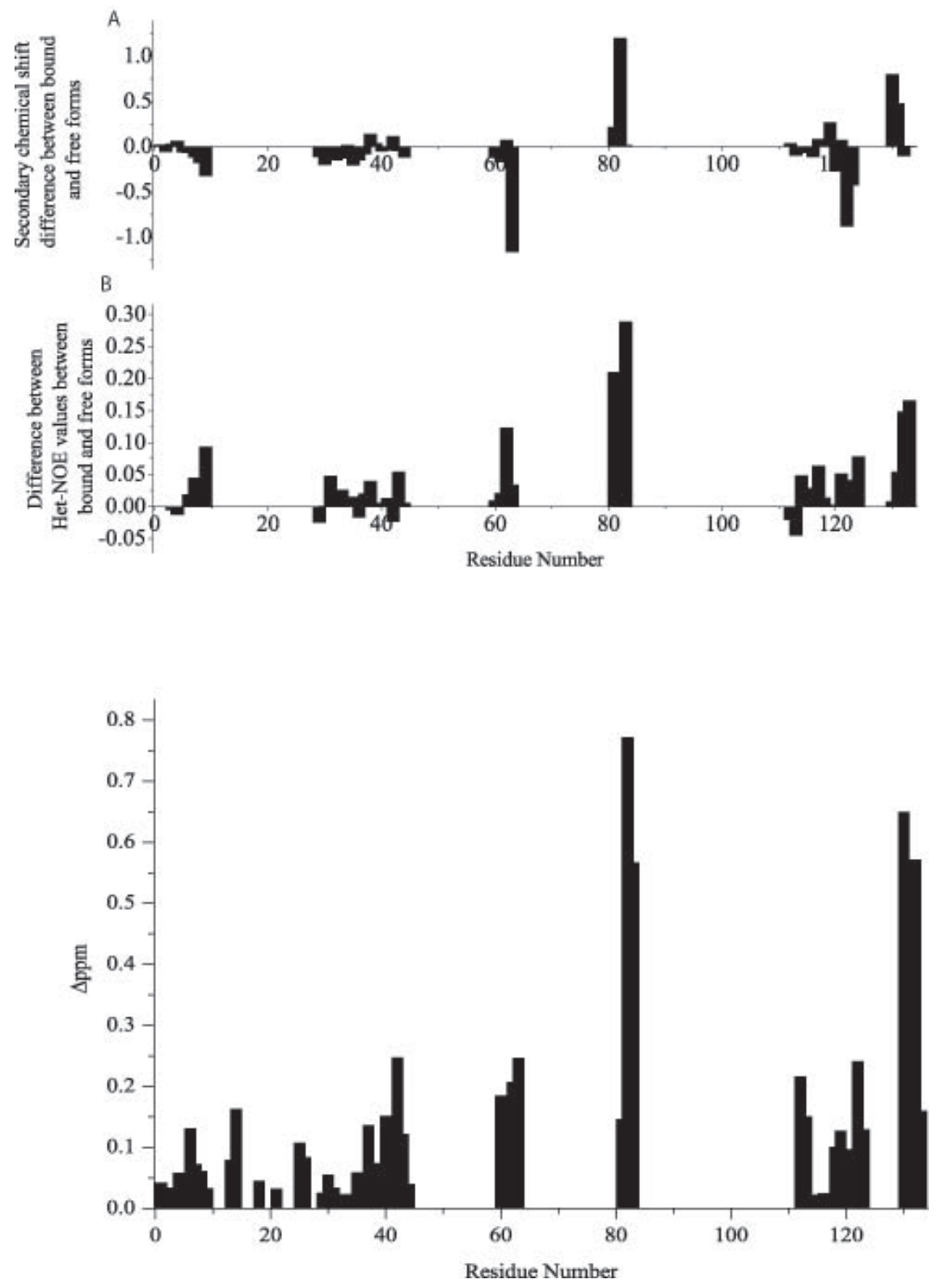

Figure 4.36: Structural and dynamic changes in CitAP upon binding to citrate as monitored by multidimensional NMR spectroscopy. Top panel: Difference between secondary $\mathrm{C}_{\alpha} / \mathrm{C}$ chemical shifts (secondary chemical shifts $=$ difference between experimental chemical shifts and random coil values) in the citrate-bound and the citrate-free state. Note the significant increase in helical propensity for residues 129-130. Middle panel: Difference between ${ }^{1} \mathrm{H}-{ }^{15} \mathrm{~N}$ NOE values between the citrate-bound and citrate-free state. Overall the ${ }^{1} \mathrm{H}-{ }^{15} \mathrm{~N}$ NOE values were slightly increased indicating a global increase in the rigidity of the backbone upon binding to citrate. The strongest increase was observed for residues neighboring the major loop and for residues 129-130. Bottom panel: Difference in ${ }^{1} \mathrm{H} /{ }^{15} \mathrm{~N}$ chemical shifts between citrate-bound and citrate-free state. 
changes were observed in the $\mathrm{C}$ terminal region and the part of the major loop suggesting an increased stability of the bound form in these regions of the protein. Large chemical shift changes were also observed in the major loop regions (residues 81-84) and the $\mathrm{C}$ terminal (residues 130-133) as seen in Figure 4.36 bottom panel (also see the HSQC spectrum in Figure 4.35). Difference in secondary chemical shift of the assigned residues of the citrate bound and free form also indicate changes in the major loop region and the $\mathrm{C}$ terminus region of the protein (Figure 4.36 top panel). Significant increase in helical propensity was observed for residues 129-131 corresponding to the $3_{10}$ helix in the citrate bound crystal structure. Large ${ }^{1} \mathrm{H} /{ }^{15} \mathrm{~N}$ chemical shift differences are also observed in the major loop region and $\mathrm{C}$ terminal region of the protein (Figure 4.36 bottom panel). These observations suggest structural and dynamic changes in the major loop region and the $\mathrm{C}$ terminal region when citrate is bound to CitAP.

\subsubsection{Mechanism of Signal Transduction}

The structural differences found in this study between the citrate-bound and citrate-free structures of CitAP give for the first time a more detailed view of potential signaling mechanisms for this histidine kinase. The open minor loop and the extended S4-S5 structure seen in the citrate-free structure is formed in a region that experiences only weak, water mediated lattice contacts, and the multiple main-chain interactions stabilizing the extended structure suggest that it is likely to reflect the pre-signaling conformation of the receptor. In the citrate-bound molecules, no lattice contacts are found around the C-terminus, and only a few distinct crystal contacts are formed around this region in the citrate-free molecules. Therefore the fundamental observation is that in the citrate-bound PAS domain, the end of the $\beta$ - sheet is pulled in towards the citrate-binding site, forming tight interactions with the activating ligand. This disrupts the strand-to-strand interactions between S4 and S5 and simultaneously pulls the C-terminus upward, away from the membrane. Regardless of the exact geometry of the C-terminal attachment, these changes are likely to be communicated directly to the TM2 helix (Figure 4.34 B and 4.37a), causing an immediate transduction of ligand binding into a conformational change at the transmembrane junction. At the Nterminus the first helical turn of helix $\mathrm{H} 1$ in the citrate-free CitAP structure unwinds to 
A

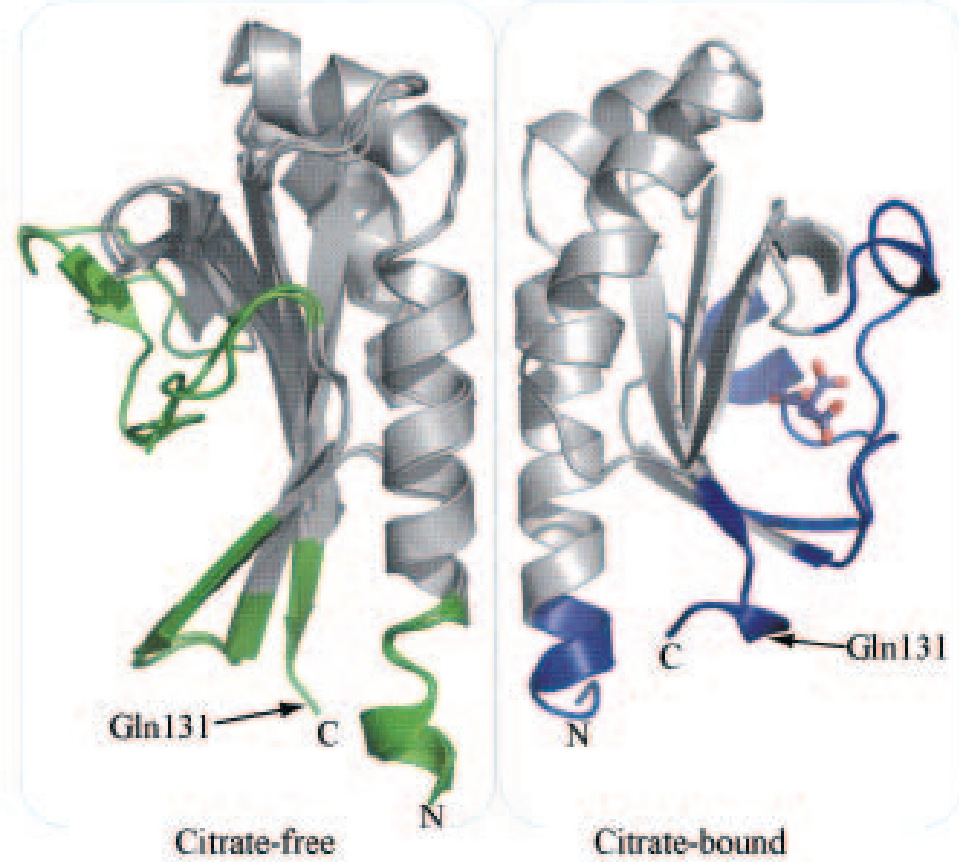

B

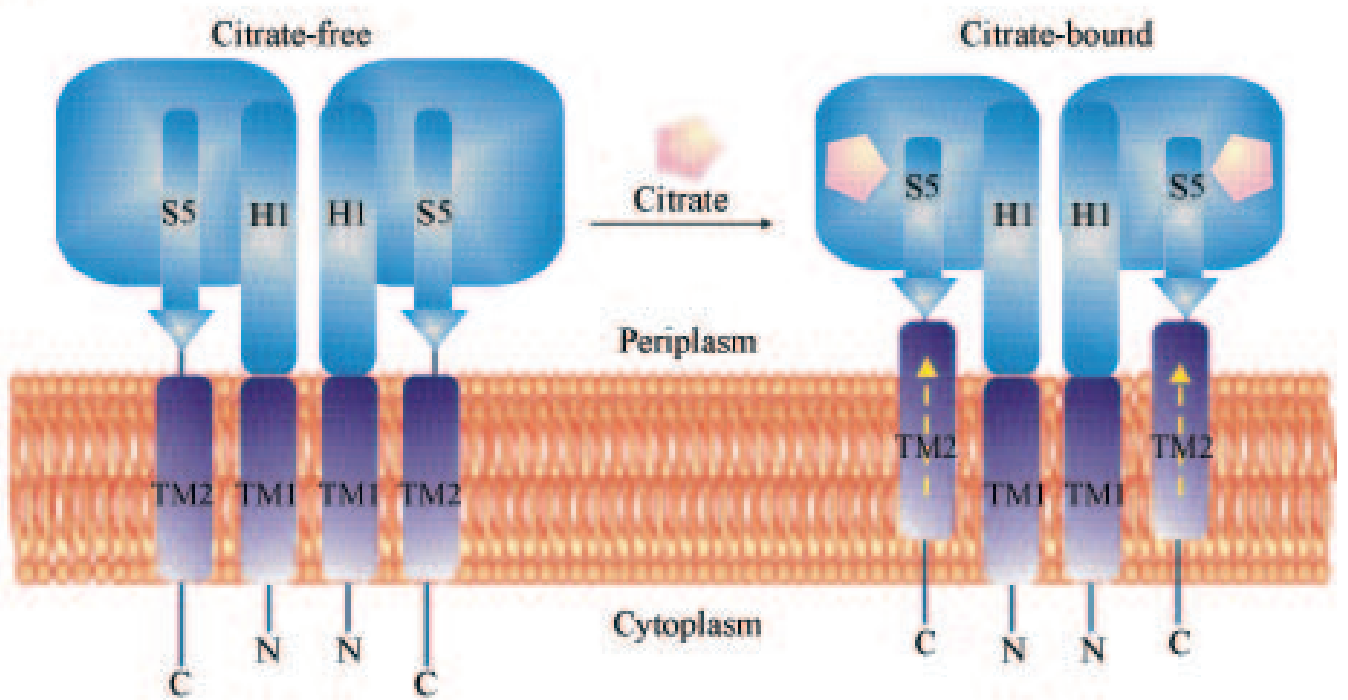

Figure 4.37: Signal transduction mechanism in CitA. (a) Comparison of the citrate-free monomer (superposed on the Citrate-bound-B) with the Citrate-bound-A monomer. (b) Model of the ligandinduced switch triggering a vectorial movement of TM2. 
an extended loop in our citrate-bound CitAP structure. An immediate connection of this structural change to citrate binding is not obvious. It is rather probable that the unwinding is due to the missing connection of helix H1 to TM1, and to the formation of extensive lattice contacts involving residues 4-12 in the citrate-free structure. Attempts to express CitAP with a more extended N-terminus were not successful. We assume that the N-terminal helix as an extension of TM1 most probably serves as an anchor keeping the periplasmic sensor domain in a stable position regarding its distance to the membrane surface. The contraction of CitAP upon ligand binding might then result in a vectorial movement of TM2 towards the sensory domain, constituting the trigger of the kinase activity (Figure $4.37 \mathrm{a}$ and $\mathrm{b}$ ). This piston-type movement of TM2 relative to TM1 has been suggested as a possible motion type of helices in membranes [153, 154]. Experimental evidence for the piston model was found for the trans membrane signaling of the aspartate receptor [155].

\subsubsection{Comparison of structures of DcuS-PD and CitAP}

DcuS of E.Coli and CitA of Klebsilla Pneumonia are homologous proteins. DcuS senses a wide range of $\mathrm{C} 4$ dicarboxylates while CitA is highly specific for citrate. Their periplasmic domains have $25 \%$ sequence identity and $70 \%$ sequence homology. The structures of DcuSPD and CitAP are similar and reveal a PAS fold. The backbone r.m.s.d for DcuS-PD structure with citrate bound and citrate free monomer $\mathrm{A}$ and $\mathrm{B}$ are $3.5 \AA, 4.09 \AA$ and 4.38 $\AA$ respectively (see Figure 4.38 ). Except for the small beta strand (S2) in citrate bound CitAP, secondary structural elements of citrate bound CitAP and DcuS-PD are conserved. In DcuS-PD, the $\mathrm{N}$ terminal helix is slightly bent inward with the beta sheets. Binding site for ligands in DcuS-PD and CitAP are conserved. As seen from the Figure 4.38, the binding site is more open in DcuS-PD than citrate bound structure of CitAP, but is less so from the citrate free CitAP. Consequently the $\beta$ sheets are less bent in the DcuS-PD structure compared to citrate bound CitAP. The residues of DcuS-PD affected by fumarate binding are located in the 4 main $\beta$ strands and in the major and minor loops. In CitAP the residues having direct hydrogen bonds with citrate are also located in the four $\beta$ strands and the major and minor loops. The residue showing major peak intensity increase on binding of fumarate is Q144 which is in the minor loop region of DcuS-PD. The largest chemical shift 

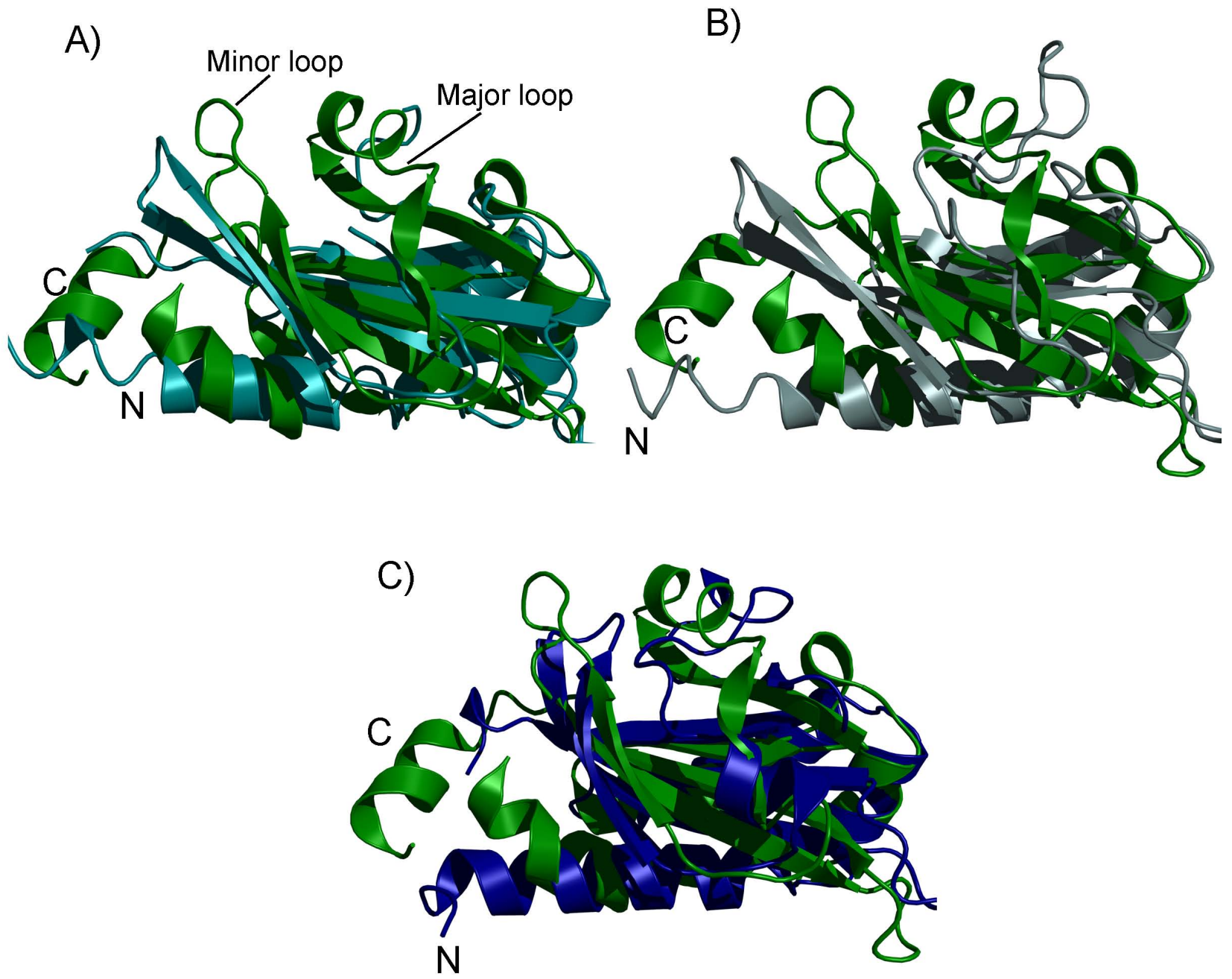

Figure 4.38: Comparison of the DcuS-PD structure with citrate-free monomer and citrate-bound monomer structures. A) Superposition of the DcuS-PD structure (green) with the citrate free monomer A structure of CitAP (cyan), r.m.s.d : $4.09 \AA$. B) Superposition of the DcuS-PD structure (green) with the citrate free monomer B structure of CitAP (pale cyan), r.m.s.d : $4.38 \AA$. C) Superposition of the DcuS-PD structure (green) with the citrate bound structure of CitAP (blue), r.m.s.d : $3.5 \AA$. Notable difference are in the minor loop and the $\mathrm{N}$ and $\mathrm{C}$ terminal regions. The $\mathrm{N}$ terminal helix of DcuS-PD is curved in with the beta sheets and helix at the $\mathrm{C}$ terminal region is well formed in the case of DcuS-PD. The structure of DcuS-PD resembles the citrate bound structure of CitAP, with the binding pocket slightly more open than the citrate bound structure of CitAP. 


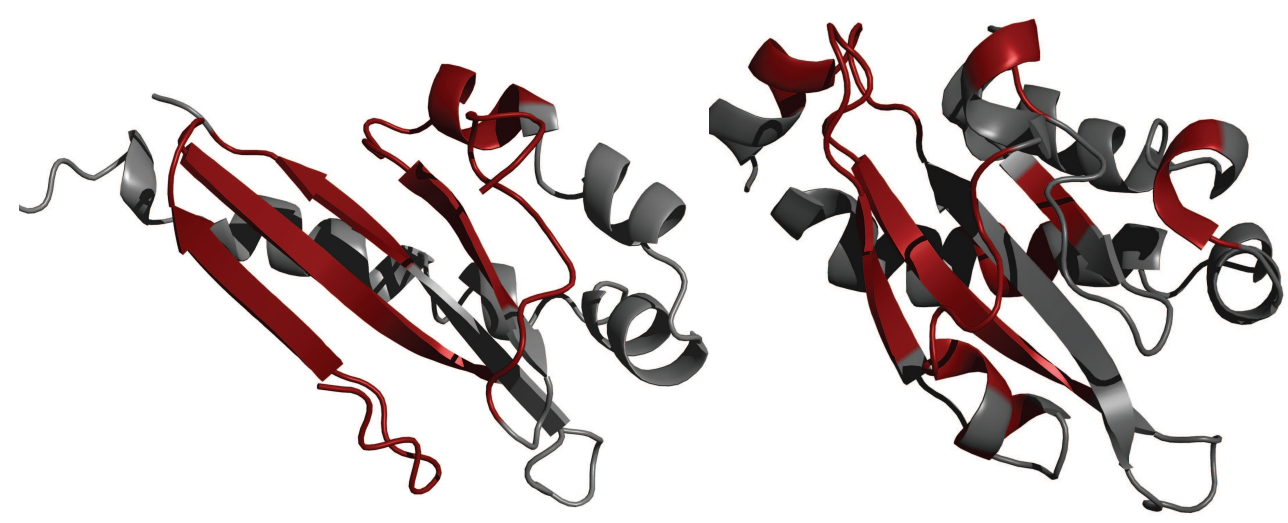

Figure 4.39: Citrate free CitAP is characterized by major line broadening due to conformational exchange. The unassigned residues are plotted on the structure of citrate free monomer A of CitAP(left). DcuS-PD also show line broadening. Residues of DcuS-PD showing very low peak intensity in the HSQC are plotted on the NMR structure of DcuS-PD (right).

change upon tartrate addition was observed for residue G140 which again is in the minor loop region in the structure of DcuS-PD. This is in accordance with the large conformational differences in the minor loop region between the citrate-free and citrate-bound CitAP X-ray structure.

Characteristic of citrate free CitAP in solution is the severe line broadening (missing peaks) in the ${ }^{15} \mathrm{~N}-{ }^{1} \mathrm{H}$ HSQC spectrum due to conformational exchange. These missing peaks are mostly from residues in the first three $\beta$ strands and the part of the major loop region and helix H3 (residues from 64-111). Dcus-PD also showed chemical exchange broadening. ${ }^{15} \mathrm{~N}-{ }^{1} \mathrm{H}$ HSQC spectrum of DcuS-PD showed very small peak intensities for a number of residues. Het-NOE values of these residue were in the 0.7 to 0.8 range, very similar to rest of the residues in the sequence, suggesting a rigid structure in these parts of the protein. Residues corresponding to the peaks showing small intensities are mapped onto the structure of DcuS-PD. This region in the structure of DcuS-PD is similar to the unassigned regions in citrate free CitAP (Figure 4.39) . 


\subsubsection{Conclusion}

In this section, the NMR solution studies on the periplasmic domain of CitA (CitAP) with and without the ligand are presented. In the course of this study the X-ray crystallographic structures of CitAP with and without the ligand were determined. The description of these structures is also presented.

The X-ray structures reveal a PAS fold similar to DcuS-PD and other PAS domains. The citrate bound structure was determined without the non physiological molybdate which was present in the previously determined X-ray structure.

There were two conformers of CitAP (monomer A and monomer B) in the asymmetric unit of the citrate free CitAP crystal structure. A part of the major loop could not be traced in monomer $\mathrm{A}$ and the $\mathrm{C}$ terminal residues were missing in monomer $\mathrm{B}$. This suggests that the citrate-free CitAP is flexible in the $\mathrm{C}$ terminal and also in some parts the major loop region. But fortunately specific conformational changes were observed between the structures of the citrate-free and -bound form. Based on this, a possible mechanism for signal transduction is discussed.

By NMR titration it was shown that in solution molybdate might induce a different structural response than with only citrate bound to CitAP. Molybdate also induces aggregation of the protein. The newly determined bound structure did indeed differ in the dimer interface and slight change in the binding of citrate to CitAP from previously published X-ray structure. Previous studies suggest that CitAP might also sense sodium ion. A sodium ion was tentatively localized in the citrate bound structure of CitAP. With NMR titrations, this is found to be true in solution as well.

NMR backbone assignments for $90 \%$ of the peaks seen in the ${ }^{15} \mathrm{~N}-{ }^{1} \mathrm{H}$ HSQC were obtained for both the bound and free form of CitA. The NMR solution structures of CitAP could not be determined because of the large number of missing peaks in NMR spectra due to chemical/conformational exchange broadening. Nevertheless, with the available assignments, the chemical shift difference and the secondary structural propensity difference between the free and the bound forms of the CitAP protein were determined. These values indicate significant difference in chemical shifts and secondary structural propensities in the C-terminal regions 
of the protein. The model proposed here for signal transduction is based on the structures of the citrate free and bound CitAP. This is also consistent with the present NMR data and the previous knowledge of signal transduction mechanisms.

Correlation co-efficients from residual dipolar coupling analysis of citrate free CitAP suggest that monomer A might be relatively more closer to the solution structure than monomer B. The correlation coefficient of RDCs of citrate free CitAP fits even better with the citrate bound structure of CitAP. RDCs of citrate-free CitAP therefore have a major contribution from the citrate-bound CitAP structure (RDC analysis indicate that the population of citrate bound structure would be $80 \%$ and that of the monomer $\mathrm{A}$ of citrate free form to be $20 \%$ ). This suggest that the binding pocket of citrate is already formed in the citrate free solution of CitAP. CitAP might require the two transmembrane helices to keep the N- and C-terminal parts of the protein together, and without the transmembrane part the protein is not confined to one conformational space. Smaller Het-NOE values in the N- and C-terminal regions indicate greater flexibility in these regions of the protein, hence providing greater freedom for the protein to sample different conformational space. 


\section{Chapter 5}

\section{Summary and outlook}

The ability of organisms to adapt to changing environmental conditions is essential for their long-term survival and requires a set of receptors that can recognize external stimuli and activate the appropriate biochemical responses. In bacteria, two-component regulatory systems play an important role in these processes. In particular, membrane-bound sensor histidine kinases allow the detection of ligands outside of the cytoplasmic membrane, so that the cellular response does not require transport or diffusion of these ligands into the cell. However, despite their key role in bacterial adaptation, many aspects of the molecular mechanisms of these proteins are unknown. In this thesis a detailed NMR studies, complemented by crystallographic results on two sensory periplasmic domain of histidine kinases, DcuS and CitA, are presented. DcuS of E.Coli and CitA of Klebsiella pneumoniae are homologous proteins involved in sensing different types of $\mathrm{C} 4$ di-carboxylates. While CitA is highly specific for citrate, DcuS senses a wide range of $\mathrm{C} 4$ dicarboxylates with much lower affinity.

NMR solution structure of the ligand free periplasmic domain of DcuS was determined. The structure of the periplasmic domain was refined using RDCs. In order to measure all types of RDCs in the committed time in which the protein was stable, a simple strategy for simultaneous measurement of different types of RDCs with minimum resonance overlap is also presented. ${ }^{15} \mathrm{~N}-{ }^{1} \mathrm{H}$ HSQC based titrations with fumarate and tartrate were performed to determine the binding pocket in DcuS-PD. Binding of fumarate resulted in sharpening of peaks and tartrate binding resulted in small chemical shift changes in NMR spectrum for residues in the binding pocket of the structure. However no such changes were observed for residues outside the binding region as it would have been expected for a signal transfer from the periplasmic domain to transmembrane part. 
NMR studies on periplasmic domain of CitA was motivated by the fact that citrate binds to CitAP with a higher affinity than any of the $\mathrm{C} 4$ di carboxylates binding to DcuS-PD. The higher affinity and the higher specificity of CitAP for citrate could lead to a more clearer difference in the conformation of ligand free and bound form of the periplasmic domain. So far the X-ray structure of citrate bound CitAP was known. At first, a citrate free CitAP structure would be required to obtain a possible conformational changes which would allow the description of the signal transduction process. The first aim of this study focuses on obtaining the structure of citrate free CitAP. The X-ray structure of the citrate bound form that existed prior to this study was obtained with the exogenous histidine tag still attached to the protein. Moreover, two types of molybdate moieties were found bound to the protein in the citrate binding pocket and at the protein dimer interface. To minimize the risk of misleading interpretation, this work includes the study of the influence of the histidine tag and molybdate in CitAP. There after major part of the work is dedicated to the structural and dynamic behavior of citrate free and bound CitAP without histidine tag and molybdate.

Due to conformational broadening only about $62 \%$ of the peaks were observed in the ${ }^{15} \mathrm{~N}$ ${ }^{1} \mathrm{H}$ HSQC spectrum of citrate free CitAP and $83 \%$ peaks were present in the citrate bound CitAP HSQC spectrum. Because of the large number of missing peaks, the NMR solution structure of the citrate free and bound form of CitAP could not be determined. However the X-ray structure of the citrate free and bound form of CitAP could be determined. The structure has a PAS fold, very similar to DcuS-PD structure. The binding pocket is formed by the $\beta$ sheet of the PAS fold and the inter-strand minor and major loops. The binding pocket is very similar to the $\mathrm{C} 4$ di-carboxylate binding pocket observed in DcuS-PD. In the citrate bound structure a $\mathrm{Na}^{+}$ion was tentatively localized between $\mathrm{N}$ terminal helix and the $\beta$ sheets. This was also confirmed by NMR titrations. Hence CitAP may be involved in sensing both citrate and $\mathrm{Na}^{+}$ion in solution.

There are two conformers of citrate free monomer structures in the asymmetric unit of CitAP. Specific conformational change can be observed between the citrate free and citrate bound structures of CitAP. The major conformational differences are seen in the binding 
pocket and also in the C-terminal region of the protein. The minor loop and the major loop (which defines the binding pocket of citrate) are in an open conformation in the citrate free structure while it is more closed in the citrate bound structure. The $\mathrm{C}$ terminal $3_{10}$ helix in the bound structure is absent in the citrate free structure. In solution, definite helical propensity is observed in the $\mathrm{C}$ - terminal region in the citrate bound-CitAP. Large chemical shift changes are observed in the C-terminal and major loop region of the protein. A model of signal transfer is described using the conformational differences seen in the crystal structures. A vectorial movement of the trans-membrane helix attached to the C-terminus of the protein could be used for the signal transduction cascade. This model is consistent with the model of signal transduction described for aspartate sensor.

A part of the major loop region and also the C-terminal part of the protein could not be traced in one of the two monomer structures. In solution, the citrate free form of CitAP is a monomer. C-terminal residues and the the three residues that could be assigned in the major loop region showed lower Het-NOE values, indicating higher flexibility. Surprisingly the RDCs measured for citrate free CitAP fit better with citrate bound structure of CitAP. RDC analysis also indicate that in citrate free CitAP solution, larger contribution to RDCs come from the citrate bound conformer (around 80\%), compared to citrate free monomer A (20\%) conformer. .

The first step in the signal transduction mechanism would require the signal from the binding pocket to be transferred to the C-terminal part of the periplasmic domain. Hence there should exist a conserved motion of residues between the binding pocket and the Cterminal part of the protein which allows for the signal transfer to occur. This would mean that a different conformational state of protein in either end of the signal transfer process will have a complementary effect on the other end. Since the transmembrane part of the protein are absent in the present study, the $\mathrm{N}$ - and C-terminal part of the periplasmic domain are not fixed onto the membrane. This makes the $\mathrm{N}$ and C-terminal residues highly flexible (as seen from lower Het-NOE values). The flexibility of the $\mathrm{N}$ - and C-terminal region therefore provides more conformational freedom to the protein. On the other hand if the protein is 
fixed on the membrane, there would be limited mobility in the $\mathrm{N}$ and $\mathrm{C}$ terminal region and that would make the protein sample a narrow conformational space. In the cases studied here, the conformational freedom due to absence of transmembrane part of the protein could explain the severe line broadening observed in the NMR spectra of citrate free form of CitAP. From RDC analysis it seems that the major conformer in the citrate free CitAP solution would be the bound form of CitAP. Stability in the binding pocket also leads to stability in the $\mathrm{N}$ and $\mathrm{C}$ terminal region of the protein. Addition of citrate populates the citrate bound conformer which was already present in solution and makes the overall structure more stable (indicated by sharpening of peaks and Het-NOE values of 0.8 for most of the residues). The sharpening of peaks as seen in the fumarate titration of DcuS-PD also arises as a result of selectively populating one of the conformers in the DcuS-PD fumarate free solution. In such systems the bound conformers seems to be more populated as it is less dynamic and more stable.

A better picture for signal transduction mechanism could be obtained if in the free form of the protein, the $\mathrm{N}$ - and C-terminal regions are made less mobile. Cysteine mutation with a disulphide linkage between the $\mathrm{N}$ and C-terminal parts of the protein may stabilize the protein in the ligand free form. It would be interesting to see the difference in the structure of such a system with ligand free and bound structure described here. Like in aspartate sensor, the disulphide linkage would most likely resemble the ligand bound structure than the ligand free structure. The solid state NMR studies of DcuS reconstituted in liposomes with their transmembrane part intact would be very useful to corroborate the signal transduction mechanism described here. 


\section{References}

[1] F. Bloch. Nuclear induction. Physical Review, 70(7-8):460-474, 1946.

[2] E. M. Purcell, H. C. Torrey, and R. V. Pound. Resonance absorption by nuclear magnetic moments in a solid. Physical Review, 69(1-2):37-38, 1946.

[3] N. F. Ramsey and E. M. Purcell. Interactions between nuclear spins in molecules. Physical Review, 85(1):143-144, 1952.

[4] M. P. Williamson, T. F. Havel, and K. Wuthrich. Solution conformation of proteinase Inhibitor-IIa from bull seminal plasma by $\mathrm{H}^{1}$ nuclear magnetic-resonance and distance geometry. Journal of Molecular Biology, 182(2):295-315, 1985.

[5] R. R. Ernst and W. A. Anderson. Sensitivity enhancement in magnetic resonance .2. Investigation of intermediate passage conditions. Review of Scientific Instruments, 36(12):1696, 1965.

[6] W. P. Aue, E. Bartholdi, and R. R. Ernst. 2-Dimensional spectroscopy - Application to nuclear magnetic-resonance. Journal of Chemical Physics, 64(5):2229-2246, 1976.

[7] R. H. Griffey and A. G. Redfield. Proton-detected heteronuclear edited and correlated nuclear-magnetic-resonance and nuclear overhauser effect in solution. Quarterly Reviews of Biophysics, 19(1-2):51-82, 1987.

[8] A. P. Hansen, A. M. Petros, A. P. Mazar, T. M. Pederson, A. Rueter, and S. W. Fesik. A practical method for uniform isotopic labeling of recombinant proteins in mammalian-cells. Biochemistry, 31(51):12713-12718, 1992.

[9] T. Kigawa, Y. Muto, and S. Yokoyama. Cell-free synthesis and amino acid-selective stable-isotope labeling of proteins for NMR analysis. Journal of Biomolecular NMR, 6(2):129-134, 1995. 
[10] J. W. Lustbader, S. Birken, S. Pollak, A. Pound, B. T. Chait, U. A. Mirza, S. Ramnarain, R. E. Canfield, and J. M. Brown. Expression of human chorionic gonadotropin uniformly labeled with NMR isotopes in Chinese hamster ovary cells: An advance toward rapid determination of glycoprotein structures. Journal of Biomolecular NMR, 7(4):295-304, 1996.

[11] L. P. McIntosh, E. Brun, and L. E. Kay. Stereospecific assignment of the $\mathrm{NH}_{2}$ resonances from the primary amides of asparagine and glutamine side chains in isotopically labeled proteins. Journal of Biomolecular NMR, 9(3):306-312, 1997.

[12] L. P. McIntosh, F. W. Dahlquist, and A. G. Redfield. Proton NMR and NOE structural and dynamic studies of larger proteins and nucleic-acids aided by isotope labels - T4 Lysozyme. Journal of Biomolecular Structure and Dynamics, 5(1):21-34, 1987.

[13] D. C. Muchmore, L. P. McIntosh, C. B. Russell, D. E. Anderson, and F. W. Dahlquist. Expression and ${ }^{15} \mathrm{~N}$ labeling of proteins for proton and ${ }^{15} \mathrm{~N}$ nuclear-magnetic-resonance. Methods in Enzymology, 177:44-73, 1989.

[14] B. J. Stockman, M. D. Reily, W. M. Westler, E. L. Ulrich, and J. L. Markley. Concerted two-dimensional NMR Approaches to ${ }^{1} \mathrm{H},{ }^{13} \mathrm{C}$, and ${ }^{15} \mathrm{~N}$ resonance assignments in proteins. Biochemistry, 28(1):230-236, 1989.

[15] R. A. Venters, W. J. Metzler, L. D. Spicer, L. Mueller, and B. T. Farmer. Use of ${ }^{1} \mathrm{H}^{N}{ }_{-}^{1} \mathrm{H}^{N}$ NOEs to determine protein global folds in perdeuterated proteins. Journal of the American Chemical Society, 117(37):9592-9593, 1995.

[16] W. M. Westler, B. J. Stockman, and J. L. Markley. Correlation of ${ }^{13} \mathrm{C}$ and ${ }^{15} \mathrm{~N}$ chemicalshifts in selectively and uniformly labeled proteins by heteronuclear two-dimensional NMR-spectroscopy. Journal of the American Chemical Society, 110(18):6256-6258, 1988.

[17] C. Griesinger, O. W. Sorensen, and R. R. Ernst. A practical approach to 3-dimensional NMR-spectroscopy. Journal of Magnetic Resonance, 73(3):574-579, 1987. 
[18] C. Griesinger, O. W. Sorensen, and R. R. Ernst. 3-Dimensional fourier spectroscopy - application to high-resolution NMR. Journal of Magnetic Resonance, 84(1):14-63, 1989.

[19] L. E. Kay, G. M. Clore, A. Bax, and A. M. Gronenborn. 4-Dimensional heteronuclear triple-resonance NMR-spectroscopy of Interleukin-1- $\beta$ in solution. Science, 249(4967):411-414, 1990.

[20] M. Sattler, J. Schleucher, and C. Griesinger. Heteronuclear multidimensional NMR experiments for the structure determination of proteins in solution employing pulsed field gradients. Progress in Nuclear Magnetic Resonance Spectroscopy, 34(2):93-158, 1999.

[21] C. Griesinger, M. Hennig, J. P. Marino, B. Reif, C. Richter, and H. Schwalbe. Modern techniques in protein NMR series, 16. Kluwer Academics, New York 1998, 1999.

[22] J. H. Prestegard. New techniques in structural NMR - Anisotropic interactions. Nature Structural Biology, 5:517-522, 1998.

[23] G. A. Morris and R. Freeman. Enhancement of nuclear magnetic-resonance signals by polarization transfer. Journal of the American Chemical Society, 101(3):760-762, 1979.

[24] O. W. Sorensen, G. W. Eich, M. H. Levitt, G. Bodenhausen, and R. R. Ernst. Product operator-formalism for the description of NMR pulse experiments. Progress in Nuclear Magnetic Resonance Spectroscopy, 16:163-192, 1983.

[25] J. Schleucher, M. Sattler, and C. Griesinger. Coherence selection by gradients without signal attenuation - Application to the 3-Dimensional HNCO experiment. Angewandte Chemie-International Edition in English, 32(10):1489-1491, 1993.

[26] L. E. Kay, P. Keifer, and T. Saarinen. Pure absorption gradient enhanced heteronuclear single quantum correlation spectroscopy with improved sensitivity. Journal of the american chemical society, 114(10):10663-10665, 1992. 
[27] M. Sattler, M. G. Schwendinger, J. Schleucher, and C. Griesinger. Novel Strategies for sensitivity enhancement in heteronuclear multidimensional NMR experiments employing pulsed-field gradients. Journal of Biomolecular NMR, 6(1):11-22, 1995.

[28] M. Sattler, P. Schmidt, J. Schleucher, O. Schedletzky, S. J. Glaser, and C. Griesinger. Novel pulse sequences with sensitivity enhancement for in-phase coherence transfer employing pulsed-field gradients. Journal of Magnetic Resonance Series B, 108(3):235$242,1995$.

[29] K. Wuthrich. NMR of protein and nucleic acids. Wiley Interscience, NewYork, 1986.

[30] Sharon J. Archer, Mitsuhiko Ikura, Dennis A. Torchia, and Ad Bax. An alternative 3D NMR technique for correlating backbone ${ }^{15} \mathrm{~N}$ with side chain $\mathrm{H} \beta$ resonances in larger proteins. Journal of Magnetic Resonance (1969), 95(3):636-641, 1991.

[31] J. Cavanagh, A. G. Palmer, P. E. Wright, and M. Rance. Sensitivity improvement in proton-detected 2-Dimensional heteronuclear relay spectroscopy. Journal of Magnetic Resonance, 91(2):429-436, 1991.

[32] J. R. Tolman, J. M. Flanagan, M. A. Kennedy, and J. H. Prestegard. Nuclear magnetic dipole interactions in field-oriented proteins - Information for structure determination in solution. Proceedings of the National Academy of Sciences of the United States of America, 92(20):9279-9283, 1995.

[33] N. Tjandra and A. Bax. Direct measurement of distances and angles in biomolecules by NMR in a dilute liquid crystalline medium. Science, 278(5340):1111-1114, 1997.

[34] N. Tjandra. Establishing a degree of order: Obtaining high-resolution NMR structures from molecular alignment. Structure with Folding and Design, 7(9):R205-R211, 1999.

[35] C. R. Sanders and J. H. Prestegard. Magnetically orientable phospholipid-bilayers containing small amounts of a bile-salt analog, CHAPSO. Biophysical Journal, 58(2):447460, 1990. 
[36] C. R. Sanders and J. P. Schwonek. Characterization of magnetically orientable bilayers in mixtures of Dihexanoylphosphatidylcholine and Dimyristoylphosphatidylcholine by solid-state NMR. Biochemistry, 31(37):8898-8905, 1992.

[37] M. R. Hansen, L. Mueller, and A. Pardi. Tunable alignment of macromolecules by filamentous Phage yields dipolar coupling interactions. Nature Structural Biology, 5(12):1065-1074, 1998.

[38] M. Ruckert and G. Otting. Alignment of biological macromolecules in novel nonionic liquid crystalline media for NMR experiments. Journal of the American Chemical Society, 122(32):7793-7797, 2000.

[39] R. Tycko, F. J. Blanco, and Y. Ishii. Alignment of biopolymers in strained gels: A new way to create detectable dipole-dipole couplings in high-resolution biomolecular NMR. Journal of the American Chemical Society, 122(38):9340-9341, 2000.

[40] M. P. Nieh, C. J. Glinka, S. Krueger, R. S. Prosser, and J. Katsaras. Sans study of the structural phases of magnetically alignable Lanthanide-Doped phospholipid mixtures. Langmuir, 17(9):2629-2638, 2001.

[41] A. Bax, G. Kontaxis, and N. Tjandra. Dipolar couplings in macromolecular structure determination. Method Enzymology, 339:127-174, 2001.

[42] A. Saupe. Recent results in field of liquid crystals. Angewandte Chemie-International Edition, 7(2):97, 1968.

[43] J. A. Losonczi, M. Andrec, M. W. F. Fischer, and J. H. Prestegard. Order matrix analysis of residual dipolar couplings using singular value decomposition. Journal of Magnetic Resonance, 138(2):334-342, 1999.

[44] L. Pappalardo, I. G. Janausch, V. Vijayan, E. Zientz, J. Junker, W. Peti, M. Zweckstetter, G. Unden, and C. Griesinger. The NMR structure of the sensory domain of the membranous two-component Fumarate sensor (histidine protein kinase) DcuS of Escherichia Coli. Journal of Biological Chemistry, 278(40):39185-39188, 2003. 
[45] L. Holm and C. Sander. Protein-structure comparison by alignment of distance matrices. Journal of Molecular Biology, 233(1):123-138, 1993.

[46] Y. S. Jung, M. Sharma, and M. Zweckstetter. Simultaneous assignment and structure determination of protein backbones by using NMR dipolar couplings. Angewandte Chemie-International Edition, 43(26):3479-3481, 2004.

[47] R. Koradi, M. Billeter, and K. Wuthrich. MolMol: A program for display and analysis of macromolecular structures. Journal of Molecular Graphics, 14(1):51-55, 1996.

[48] F. Delaglio, S. Grzesiek, G. W. Vuister, G. Zhu, J. Pfeifer, and A. Bax. NmrPipe - a multidimensional spectral processing system based on unix pipes. Journal of Biomolecular NMR, 6(3):277-293, 1995.

[49] M. Zweckstetter and A. Bax. Prediction of sterically induced alignment in a dilute liquid crystalline phase: Aid to protein structure determination by NMR. Journal of the American Chemical Society, 122(15):3791-3792, 2000.

[50] A. Bax and S. Grzesiek. Methodological advances in protein NMR. Accounts of Chemical Research, 26(4):131-138, 1993.

[51] M. Salzmann, K. Pervushin, G. Wider, H. Senn, and K. Wuthrich. TROSY in tripleresonance experiments: New perspectives for sequential NMR assignment of large proteins. Proceedings of the National Academy of Sciences of the United States of America, 95(23):13585-13590, 1998.

[52] W. Peti, C. Griesinger, and W. Bermel. Adiabatic TOCSY for C,C and H,H J-transfer. Journal of Biomolecular NMR, 18(3):199-205, 2000.

[53] M. Zweckstetter and T. A. Holak. Robust refocusing of ${ }^{13} C_{\alpha}$ magnetization in multidimensional NMR experiments by adiabatic fast passage pulses. Journal of Biomolecular NMR, 15(4):331-334, 1999.

[54] M. Sattler, M. Maurer, J. Schleucher, and C. Griesinger. A simultaneous ${ }^{15} \mathrm{~N},{ }^{1} \mathrm{H}-\mathrm{HSQC}$ and ${ }^{13} \mathrm{C},{ }^{1} \mathrm{H}-\mathrm{HSQC}$ with sensitivity enhancement and a heteronuclear gradient-echo. Journal of Biomolecular NMR, 5(1):97-102, 1995. 
[55] Y. L. Xia, A. Yee, C. H. Arrowsmith, and X. L. Gao. ${ }^{1} \mathrm{H}_{C}$ and ${ }^{1} \mathrm{H}^{N}$ Total NOE correlations in a single 3D NMR experiment. ${ }^{15} \mathrm{~N}$ and ${ }^{13} \mathrm{C}$ time-sharing in $\mathrm{T}(1)$ and $\mathrm{T}(2)$ dimensions for simultaneous data acquisition. Journal of Biomolecular NMR, 27(3):193-203, 2003.

[56] F. A. A. Mulder, C. A. E. M. Spronk, M. Slijper, R. Kaptein, and R. Boelens. Improved HSQC experiments for the observation of exchange broadened signals. Journal of Biomolecular NMR, 8(2):223-228, 1996.

[57] S. Schwarzinger, G. J. A. Kroon, T. R. Foss, J. Chung, P. E. Wright, and H. J. Dyson. Sequence-dependent correction of random coil NMR chemical shifts. Journal of the American Chemical Society, 123(13):2970-2978, 2001.

[58] D. S. Wishart and B. D. Sykes. Chemical-shifts as a tool for structure determination. Methods in Enzymology, 239:363-392, 1994.

[59] W. J. Metzler, K. L. Constantine, M. S. Friedrichs, A. J. Bell, E. G. Ernst, T. B. Lavoie, and L. Mueller. Characterization of the 3-Dimensional solution structure of human Profilin $-{ }^{1} \mathrm{H}$, onec and ${ }^{15} \mathrm{~N}$ NMR assignments and global folding pattern. Biochemistry, 32(50):13818-13829, 1993.

[60] G. Cornilescu, J. L. Marquardt, M. Ottiger, and A. Bax. Validation of protein structure from anisotropic carbonyl chemical shifts in a dilute liquid crystalline phase. Journal of the American Chemical Society, 120(27):6836-6837, 1998.

[61] M. Ottiger, F. Delaglio, and A. Bax. Measurement of J and dipolar couplings from simplified two-dimensional NMR spectra. Journal of Magnetic Resonance, 131(2):373378, 1998.

[62] J. J. Chou, F. Delaglio, and A. Bax. Measurement of one-bond ${ }^{15} \mathrm{~N}-{ }^{13} \mathrm{C}$ ' dipolar couplings in medium sized proteins. Journal of Biomolecular NMR, 18(2):101-105, 2000. 
[63] V. Vijayan and M. Zweckstetter. Simultaneous measurement of protein one-bond residual dipolar couplings without increased resonance overlap. Journal of Magnetic Resonance, 174(2):245-253, 2005.

[64] N. Tjandra, A. Szabo, and A. Bax. Protein backbone dynamics and ${ }^{15} \mathrm{~N}$ chemical shift anisotropy from quantitative measurement of relaxation interference effects. Journal of the American Chemical Society, 118(29):6986-6991, 1996.

[65] G. Lipari, A. Szabo, and R. M. Levy. Protein dynamics and NMR Relaxation - comparison of simulations with experiment. Nature, 300(5888):197-198, 1982.

[66] G. Lipari and A. Szabo. Model-free approach to the interpretation of nuclear magneticresonance relaxation in macromolecules .1. Theory and range of validity. Journal of the American Chemical Society, 104(17):4546-4559, 1982.

[67] G. Lipari and A. Szabo. Model-Free approach to the interpretation of nuclear magneticresonance relaxation in macromolecules .2. Analysis of experimental results. Journal of the American Chemical Society, 104(17):4559-4570, 1982.

[68] A. A. Bothnerby. Encyclopedia of nuclear magnetic resonance. pages 2932-2938. Wiley, Chichester, 1996.

[69] C. Gayathri, A. A. Bothnerby, P. C. M. Vanzijl, and C. Maclean. Dipolar magnetic-field effects in NMR-spectra of liquids. Chemical Physics Letters, 87(2):192-196, 1982.

[70] A. Saupe and G. Englert. High-resolution nuclear magnetic resonance spectra of orientated molecules. Physical Review Letters, 11(10):462-464, 1963.

[71] J. Sass, F. Cordier, A. Hoffmann, A. Cousin, J. G. Omichinski, H. Lowen, and S. Grzesiek. Purple membrane induced alignment of biological macromolecules in the magnetic field. Journal of the American Chemical Society, 121(10):2047-2055, 1999.

[72] J. J. Chou, S. P. Li, and A. Bax. Study of conformational rearrangement and refinement of structural homology models by the use of heteronuclear dipolar couplings. Journal of Biomolecular NMR, 18(3):217-227, 2000. 
[73] F. Delaglio, G. Kontaxis, and A. Bax. Protein structure determination using molecular fragment replacement and NMR dipolar couplings. Journal of the American Chemical Society, 122(9):2142-2143, 2000.

[74] J. C. Hus, D. Marion, and M. Blackledge. Determination of protein backbone structure using only residual dipolar couplings. Journal of the American Chemical Society, 123(7):1541-1542, 2001.

[75] J. H. Prestegard, H. Valafar, J. Glushka, and F. Tian. Nuclear magnetic resonance in the era of structural genomics. Biochemistry, 40(30):8677-8685, 2001.

[76] E. de Alba, M. Suzuki, and N. Tjandra. Simple multidimensional NMR experiments to obtain different types of one-bond dipolar couplings simultaneously. Journal of Biomolecular NMR, 19(1):63-67, 2001.

[77] P. Permi, P. R. Rosevear, and A. Annila. A set of HNCO-based experiments for measurement of residual dipolar couplings in ${ }^{15} \mathrm{~N},{ }^{13} \mathrm{C},\left({ }^{2} \mathrm{H}\right)$-labeled proteins. Journal of Biomolecular NMR, 17(1):43-54, 2000.

[78] S. Heikkinen, P. Permi, and I. Kilpelainen. Methods for the measurement of ${ }^{1} \mathrm{~J}_{N c \alpha}$ and ${ }^{2} \mathrm{~J}_{N c \alpha}$ from a simplified ${ }^{13} C_{\alpha}$-coupled ${ }^{15} \mathrm{~N}$ Se-HSQC spectrum. Journal of Magnetic Resonance, 148(1):53-60, 2001.

[79] H. L. J. Wienk, M. M. Martinez, G. N. Yalloway, J. M. Schmidt, C. Perez, H. Ruterjans, and F. Lohr. Simultaneous measurement of protein one-bond and two-bond nitrogencarbon coupling constants using an internally referenced quantitative J-correlated $\left[{ }^{15} \mathrm{~N}\right.$, $\mathrm{H}^{1}$ ]-TROSY-HNCO experiment. Journal of Biomolecular NMR, 25(2):133-145, 2003.

[80] P. Permi. Measurement of residual dipolar couplings from ${ }^{1} \mathrm{H}_{\alpha}$ to ${ }^{13} C_{\alpha}$ and ${ }^{15} \mathrm{~N}$ using a simple HNCA-based experiment. Journal of Biomolecular NMR, 27(4):341-349, 2003.

[81] K. Y. Ding and A. M. Gronenborn. Simultaneous and accurate determination of onebond ${ }^{15} \mathrm{~N}-{ }^{13} \mathrm{C}$ ' and two-bond ${ }^{1} \mathrm{H}^{N-13} \mathrm{C}^{\prime}$ dipolar couplings. Journal of the American Chemical Society, 125(38):11504-11505, 2003. 
[82] D. W. Yang, R. A. Venters, G. A. Mueller, W. Y. Choy, and L. E. Kay. TROSY-based HNCO pulse sequences for the measurement of ${ }^{1} \mathrm{H}^{N}{ }^{15} \mathrm{~N},{ }^{15} \mathrm{~N}-{ }^{13} \mathrm{C}^{\prime},{ }^{1} \mathrm{H}^{N} \_{ }^{13} \mathrm{C},,{ }^{13} \mathrm{C}^{\prime}-$ ${ }^{13} C_{\alpha}$ and ${ }^{1} \mathrm{H}^{N-13} C_{\alpha}$ dipolar couplings in ${ }^{15} \mathrm{~N},{ }^{13} \mathrm{C}, \mathrm{H}^{2}$-labeled proteins. Journal of Biomolecular NMR, 14(4):333-343, 1999.

[83] S. Grzesiek and A. Bax. Correlating backbone amide and side-chain resonances in larger proteins by multiple relayed triple resonance NMR. Journal of the American Chemical Society, 114(16):6291-6293, 1992.

[84] J. J. Chou and A. Bax. Protein side-chain rotamers from dipolar couplings in a liquid crystalline phase. Journal of the American Chemical Society, 123(16):3844-3845, 2001.

[85] K. V. Pervushin, G. Wider, and K. Wuthrich. Single transition-to-single transition polarization transfer $(\mathrm{ST} 2-\mathrm{PT})$ in $\left[{ }^{15} \mathrm{~N},{ }^{1} \mathrm{H}\right]$-TROSY. Journal of Biomolecular NMR, 12(2):345-348, 1998.

[86] E. Kupce, J. Boyd, and I. D. Campbell. Short selective pulses for biochemical applications. Journal of Magnetic Resonance Series B, 106(3):300-303, 1995.

[87] M. S. Silver, R. I. Joseph, and D. I. Hoult. Highly selective $\pi / 2$ and $\pi$-pulse generation. Journal of Magnetic Resonance, 59(2):347-351, 1984.

[88] E. Kupce and R. Freeman. Compensation for spin-spin coupling effects during adiabatic pulses. Journal of Magnetic Resonance, 127(1):36-48, 1997.

[89] M. Zweckstetter and A. Bax. Characterization of molecular alignment in aqueous suspensions of Pf1 Bacteriophage. Journal of Biomolecular NMR, 20(4):365-377, 2001.

[90] G. Kontaxis, G. M. Clore, and A. Bax. Evaluation of cross-correlation effects and measurement of one-bond couplings in proteins with short transverse relaxation times. Journal of Magnetic Resonance, 143(1):184-196, 2000.

[91] R. H. Contreras and J. E. Peralta. Angular dependence of spin-spin coupling constants. Progress in Nuclear Magnetic Resonance Spectroscopy, 37(4):321-425, 2000. 
[92] S. Vijaykumar, C. E. Bugg, and W. J. Cook. Structure of Ubiquitin refined at $1.8 \AA$ resolution. Journal of Molecular Biology, 194(3):531-544, 1987.

[93] S. Aizawa, C. S. Harwood, and R. J. Kadner. Signaling components in bacterial locomotion and sensory reception. Journal of Bacteriology, 182(6):1459-1471, 2000.

[94] B. T. Nixon, C. W. Ronson, and F. M. Ausubel. 2-Component regulatory systems responsive to environmental stimuli share strongly conserved domains with the nitrogen assimilation regulatory genes ntrB and ntrC. Proceedings of the National Academy of Sciences of the United States of America, 83(20):7850-7854, 1986.

[95] A. J. Ninfa and B. Magasanik. Covalent modification of the glnG product, $\mathrm{NR}_{I}$, by the glnL product, $\mathrm{NR}_{I I}$, regulates the transcription of the glnALG operon in EscherichiaColi. Proceedings of the National Academy of Sciences of the United States of America, 83(16):5909-5913, 1986.

[96] J. F. Hess, K. Oosawa, N. Kaplan, and M. I. Simon. Phosphorylation of 3 proteins in the signaling pathway of bacterial chemotaxis. Cell, 53(1):79-87, 1988.

[97] J. A. Hoch. Two-component and phosphorelay signal transduction. Current Opinion in Microbiology, 3(2):165-170, 2000.

[98] L. A. Egger, H. Park, and M. Inouye. Signal transduction via the histidyl-aspartyl phosphorelay. Genes to Cells, 2(3):167-184, 1997.

[99] A. M. Stock, V. L. Robinson, and P. N. Goudreau. Two-component signal transduction. Annual Review of Biochemistry, 69:183-215, 2000.

[100] M. Levit, Y. Liu, M. Surette, and J. Stock. Active site interference and asymmetric activation in the chemotaxis protein histidine kinase CheA. Journal of Biological Chemistry, 271(50):32057-32063, 1996.

[101] Y. Yang and M. Inouye. Intermolecular complementation between 2 defective mutant signal-transducing receptors of Escherichia-Coli. Proceedings of the National Academy of Sciences of the United States of America, 88(24):11057-11061, 1991. 
[102] L. Qin, R. Dutta, H. Kurokawa, M. Ikura, and M. Inouye. A monomeric histidine kinase derived from Envz, an Escherichia Coli osmosensor. Molecular Microbiology, 36(1):24-32, 2000.

[103] S. J. Cai and M. Inouye. Spontaneous subunit exchange and biochemical evidence for trans-autophosphorylation in a dimer of Escherichia Coli histidine kinase (EnvZ). Journal of Molecular Biology, 329(3):495-503, 2003.

[104] V. L. Robinson, D. R. Buckler, and A. M. Stock. A tale of two components: A novel kinase and a regulatory switch. Nature Structural Biology, 7(8):626-633, 2000.

[105] M.Y. Galperin. Bacterial signal transduction network in a genomic perspective. Environmental Microbiology, 6(6):552-567, 2004.

[106] J. Stock. Signal transduction: gyrating protein kinases. Current Biology, 9(10):R364R367, 1999.

[107] A. M. Bilwes, L. A. Alex, B. R. Crane, and M. I. Simon. Structure of CheA, a signaltransducing histidine kinase. Cell, 96(1):131-141, 1999.

[108] T. Tanaka, S. K. Saha, C. Tomomori, R. Ishima, D. J. Liu, K. I. Tong, H. Park, R. Dutta, L. Qin, M. B. Swindells, T. Yamazaki, A. M. Ono, M. Kainosho, M. Inouye, and M. Ikura. NMR Structure of the histidine kinase domain of the E-Coli osmosensor EnvZ. Nature, 396(6706):88-92, 1998.

[109] C. Tomomori, T. Tanaka, R. Dutta, H. Y. Park, S. K. Saha, Y. Zhu, R. Ishima, D. J. Liu, K. I. Tong, H. Kurokawa, H. Qian, M. Inouye, and M. Ikura. Solution structure of the homodimeric core domain of Escherichia Coli histidine kinase EnvZ. Nature Structural Biology, 6(8):729-734, 1999.

[110] M. R. Atkinson and A. J. Ninfa. Characterization of Escherichia-Coli glnL Mutations affecting nitrogen regulation. Journal of Bacteriology, 174(14):4538-4548, 1992.

[111] L. A. Collins, S. M. Egan, and V. Stewart. Mutational analysis reveals functional similarity between NARX, a nitrate sensor in Escherichia-Coli K-12, and the methylaccepting chemotaxis proteins. Journal of Bacteriology, 174(11):3667-3675, 1992. 
[112] J. S. Fassler, W. M. Gray, C. L. Malone, W. Tao, H. Lin, and R. J. Deschenes. Activated alleles of Yeast SLN1 Increase Mcm1-dependent reporter gene expression and diminish signaling through the Hog1 osmosensing pathway. Journal of Biological Chemistry, 272(20):13365-13371, 1997.

[113] H. Park and M. Inouye. Mutational analysis of the linker region of EnvZ, an Osmosensor in Escherichia Coli. Journal of Bacteriology, 179(13):4382-4390, 1997.

[114] C. P. Ponting and L. Aravind. PAS: A multifunctional domain family comes to light. Current Biology, 7(11):R674-R677, 1997.

[115] I. B. Zhulin and B. L. Taylor. Correlation of PAS domains with electron transportassociated proteins in completely sequenced microbial genomes. Molecular Microbiology, 29(6):1522-1523, 1998.

[116] B. L. Taylor and I. B. Zhulin. PAS Domains: Internal sensors of oxygen, redox potential, and light. Microbiology and Molecular Biology Reviews, 63(2):479-+, 1999.

[117] I. B. Zhulin and B. L. Taylor. PAS domain S-boxes in archaea, bacteria and sensors for oxygen and redox. Trends in Biochemical Sciences, 22(9):331-333, 1997.

[118] J. R. Nambu, J. O. Lewis, K. A. Wharton, and S. T. Crews. The Drosophila singleminded gene encodes a helix-loop-helix protein that acts as a master regulator of CNS midline development. Cell, 67(6):1157-1167, 1991.

[119] J. L. Pellequer, K. A. Wager-Smith, S. A. Kay, and E. D. Getzoff. Photoactive Yellow Protein: A structural prototype for the three-dimensional fold of the PAS domain superfamily. Proceedings of the National Academy of Sciences of the United States of America, 95(11):5884-5890, 1998.

[120] G. E. O. Borgstahl, D. R. Williams, and E. D. Getzoff. 1.4 Angstrom structure of Photoactive Yellow Protein, a cytosolic photoreceptor - unusual fold, active-site, and chromophore. Biochemistry, 34(19):6278-6287, 1995. 
[121] M. H. Hefti, K. J. Francoijs, S. C. de Vries, R. Dixon, and J. Vervoort. The PAS fold A redefinition of the PAS domain based upon structural prediction. European Journal of Biochemistry, 271(6):1198-1208, 2004.

[122] I. G. Janausch, E. Zientz, Q. H. Tran, A. Kroger, and G. Unden. C-4-Dicarboxylate carriers and sensors in bacteria. Biochimica Et Biophysica Acta-Bioenergetics, 1553(12):39-56, 2002.

[123] I. G. Janausch, I. Garcia-Moreno, and G. Unden. Function of DcuS from Escherichia Coli as a Fumarate-stimulated histidine protein kinase in vitro. Journal of Biological Chemistry, 277(42):39809-39814, 2002.

[124] J. S. Parkinson and E. C. Kofoid. Communication modules in bacterial signaling proteins. Annual Review of Genetics, 26:71-112, 1992.

[125] E. Zientz, J. Bongaerts, and G. Unden. Fumarate regulation of gene expression in Escherichia Coli by the DcuSR (Dcusr Genes) Two-component regulatory system. Journal of Bacteriology, 180(20):5421-5425, 1998.

[126] P. Golby, S. Davies, D. J. Kelly, J. R. Guest, and S. C. Andrews. Identification and characterization of a two-component sensor-kinase and response-regulator system (DcuSDcuR) controlling gene expression in response to C4-dicarboxylates in Escherichia Coli. Journal of Bacteriology, 181(4):1238-1248, 1999.

[127] S. Kaspar, R. Perozzo, S. Reinelt, M. Meyer, K. Pfister, L. Scapozza, and M. Bott. The periplasmic domain of the histidine autokinase CitA functions as a highly specific citrate receptor. Molecular Microbiology, 33(4):858-872, 1999.

[128] T. N. Parac, B. Coligaev, E. Zientz, G. Unden, W. Peti, and C. Griesinger. Assignment of ${ }^{1} \mathrm{H},{ }^{13} \mathrm{C}$ and ${ }^{15} \mathrm{~N}$ resonances to the sensory domain of the membraneous twocomponent fumarate sensor (histidine protein kinase) DcuS of Escherichia Coli. Journal of Biomolecular NMR, 19(1):91-92, 2001. 
[129] G. W. Vuister, F. Delaglio, and A. Bax. An empirical correlation between ${ }^{1} \mathrm{~J}_{C \alpha H \alpha}$ and protein backbone conformation. Journal of the American Chemical Society, 114(24):9674-9675, 1992.

[130] M. V. Milburn, G. G. Prive, D. L. Milligan, W. G. Scott, J. Yeh, J. Jancarik, D. E. Koshland, and S. H. Kim. 3-Dimensional structures of the ligand-binding domain of the bacterial aspartate receptor with and without a ligand. Science, 254(5036):1342-1347, 1991.

[131] J. I. Yeh, H. P. Biemann, J. Pandit, D. E. Koshland, and S. H. Kim. The 3-dimensional structure of the ligand-binding domain of a wild-type bacterial chemotaxis receptor structural comparison to the cross-linked mutant forms and conformational-changes upon ligand-binding. Journal of Biological Chemistry, 268(13):9787-9792, 1993.

[132] H. Kneuper, I. G. Janausch, V. Vijayan, M. Zweckstetter, V. Bock, C. Griesinger, and G. Unden. The nature of the stimulus and of the fumarate binding site of the fumarate sensor DcuS of Escherichia Coli. Journal of Biological Chemistry, 280(21):2059620603, 2005.

[133] T. Gerharz, S. Reinelt, S. Kaspar, L. Scapozza, and M. Bott. Identification of basic amino acid residues important for citrate binding by the periplasmic receptor domain of the sensor kinase CitA. Biochemistry, 42(19):5917-5924, 2003.

[134] E. Schwarz and D. Oesterhelt. Cloning and expression of Klebsiella-Pneumoniae genescoding for citrate transport and fermentation. Embo Journal, 4(6):1599-1603, 1985.

[135] M. E. Vanderrest, E. Schwarz, D. Oesterhelt, and W. N. Konings. DNA-sequence of a citrate carrier of Klebsiella-Pneumoniae. European Journal of Biochemistry, 189(2):401-407, 1990.

[136] M. E. Vanderrest, T. Abee, D. Molenaar, and W. N. Konings. Mechanism and energetics of a citrate-transport system of Klebsiella-Pneumoniae. European Journal of Biochemistry, 195(1):71-77, 1991. 
[137] P. Dimroth and A. Thomer. Citrate transport in Klebsiella-Pneumoniae. Biological Chemistry Hoppe-Seyler, 367(8):813-823, 1986.

[138] M. Bott, M. Meyer, and P. Dimroth. Regulation of anaerobic citrate metabolism in Klebsiella-Pneumoniae. Molecular Microbiology, 18(3):533-546, 1995.

[139] M. E. Vanderrest, R. M. Siewe, T. Abee, E. Schwarz, D. Oesterhelt, and W. N. Konings. Nucleotide-sequence and functional-properties of a sodium-dependent citrate transport-system from Klebsiella-Pneumoniae. Journal of Biological Chemistry, 267(13):8971-8976, 1992.

[140] E. Laussermair, E. Schwarz, D. Oesterhelt, H. Reinke, K. Beyreuther, and P. Dimroth. The sodium-ion translocating oxaloacetate decarboxylase of Klebsiella-Pneumoniae Sequence of the integral membrane-bound subunit-beta and gamma. Journal of Biological Chemistry, 264(25):14710-14715, 1989.

[141] E. Schwarz, D. Oesterhelt, H. Reinke, K. Beyreuther, and P. Dimroth. The Sodiumion translocating oxalacetate decarboxylase of Klebsiella-Pneumoniae - Sequence of the biotin-containing alpha-subunit and relationship to other biotin-containing enzymes. Journal of Biological Chemistry, 263(20):9640-9645, 1988.

[142] G. Woehlke, E. Laussermair, E. Schwarz, D. Oesterhelt, H. Reinke, K. Beyreuther, and P. Dimroth. Sequence of the beta-subunit of oxaloacetate decarboxylase from Klebsiella-Pneumoniae - A correction of the C-terminal part. Journal of Biological Chemistry, 267(32):22804-22805, 1992.

[143] M. Bott and P. Dimroth. Klebsiella-Pneumoniae genes for Citrate Lyase and Citrate Lyase Ligase - Localization, sequencing, and expression. Molecular Microbiology, 14(2):347-356, 1994.

[144] J. A. Hoch and T.J. Silhavy. Two-component signal transduction. ASM press, Washington, DC, 1995. 
[145] R. V. Swanson, L. A. Alex, and M. I. Simon. Histidine and aspartate phosphorylation - 2-component systems and the limits of homolog. Trends in Biochemical Sciences, 19(11):485-490, 1994.

[146] S. Reinelt, E. Hofmann, T. Gerharz, M. Bott, and D. R. Madden. The Structure of the periplasmic ligand-binding domain of the sensor Kinase CitA reveals the first extracellular PAS domain. Journal of Biological Chemistry, 278(40):39189-39196, 2003.

[147] Brien R. W. O and J. R. Stern. Role of sodium in determining alternate pathways of aerobic citrate catabolism in aerobacter-aerogenes EnZ alpha keto glutarate dehydrogenase Enz citritase EnZ oxalacetate decarboxylase. Journal of Bacteriology, 99(2):389394, 1969.

[148] R. W. Obrien. Induction by sodium of citrate fermentation enzymes in KlebsiellaAerogenes. Febs Letters, 53(1):61-63, 1975.

[149] R. W. Obrien. Effect of aeration and sodium on metabolism of citrate by KlebsiellaAerogenes. Journal of Bacteriology, 122(2):468-473, 1975.

[150] P. Dimroth, P. Jockel, and M. Schmid. Coupling mechanism of the oxaloacetate decarboxylase $\mathrm{Na}^{+}$pump. Biochimica Et Biophysica Acta-Bioenergetics, 1505(1):1-14, 2001.

[151] K. M. Pos and P. Dimroth. Functional properties of the purified $\mathrm{Na}^{+}$-dependent citrate carrier of Klebsiella Pneumoniae: Evidence for asymmetric orientation of the carrier protein in proteoliposomes. Biochemistry, 35(3):1018-1026, 1996.

[152] P. Muller, S. Kopke, and G. M. Sheldrick. Is the bond-valence method able to identify metal atoms in protein structures? Acta Crystallographica Section D-Biological Crystallography, 59:32-37, 2003.

[153] M. Hulko, F. Berndt, M. Gruber, J. U. Linder, V. Truffault, A. Schultz, J. Martin, J. E. Schultz, A. N. Lupas, and M. Coles. The HAMP domain structure implies helix rotation in transmembrane signaling. Cell, 126(5):929-940, 2006. 
[154] E. E. Matthews, M. Zoonens, and D. M. Engelman. Dynamic helix interactions in transmembrane signaling. Cell, 127(3):447-450, 2006.

[155] K. M. Ottemann, W. Z. Xiao, Y. K. Shin, and D. E. Koshland. A piston model for transmembrane signaling of the aspartate receptor. Science, 285(5434):1751-1754, 1999.

[156] O. W. Sorensen, G. W. Eich, M. H. Levit, G. Bodenhausen, and R. R. Ernst. Product operator formalism for the description of NMR pulse experiments. Progress in nuclear magnetic resonance, 16:163-192, 1984. 


\section{Appendix A \\ Time evolution of product operators}

The discussion given in the following section is based on the density operator formalism [156], which permits the most convenient description of quantum mechanical system dynamics. The density operator is a mathematical description of spin system, and is given by the sum of all spins and spin states weighted by time dependent factors. $b_{s}(t)$.

$$
\sigma(t)=\sum_{S} b_{S}(t) B_{S}
$$

where $B_{S}$ s are the set of basis vectors describing the different spin states, and are given by

$$
B_{S}=2^{(q-1)} \prod_{k=1}^{N}\left(I_{k v}\right)^{a_{s k}}
$$

where $\mathrm{N}$ is the total number of spins half in the system; $k$ and $v$ are respectively, the index of the spin and $\mathrm{x}, \mathrm{y}$, or $\mathrm{z}$; $\mathrm{q}$ is the number of single spin operators $I_{x}, I_{y}$ or $I_{z}$ in the product; for these spin $a_{s k}$ is 1 .

The complete basis set $\left(B_{S}\right)$ for a system with $\mathrm{N}$ spin $1 / 2$ consists of $4^{N}$ product operators $\left(B_{S}\right)$. As an example in table A.1, the complete set of 16 product operators $\left(B_{S}\right)$ for a two spin system with $I=S=1 / 2$ are listed.

The time evolution of the density operator $\sigma(t)$ is given by the Liouville-von Neumann equation

$$
\frac{d}{d t} \sigma(t)=-i[H(t), \sigma(t)]
$$

where $H$ is the hamiltonian under which the spin density operator $\sigma(t)$ (defined by A.1) evolves.

For $H$ being constant during the time interval 0 to $t$, equation A.3 integrates to

$$
\sigma(t)=\exp (-i H t) \sigma(0) \exp (i H t)
$$




\begin{tabular}{|l|l|}
\hline \hline$q$ & Product or basis operator $\left(B_{S}\right)$ \\
\hline 0 & unity operator $\frac{1}{2} E$ \\
1 single spin operators & $I_{x}, I_{y}, I_{z}, S_{x}, S_{y}$ and $S_{z}$ \\
& \\
2 two-spin operators & $2 I_{x} S_{x}, 2 I_{x} S_{y}, 2 I_{x} S_{z}$ \\
& $2 I_{y} S_{x}, 2 I_{y} S_{y}, 2 I_{y} S_{z}$ \\
& $2 I_{z} S_{x}, 2 I_{z} S_{y}, 2 I_{z} S_{z}$ \\
\hline \hline
\end{tabular}

Table A.1: Basis operators for $q=0,1$ or 2 for an $I S$ system of spin $1 / 2$

This type of exponential operator function can be evaluated using the Baker-CampbellHausdorff(BCH) formula. The $\mathrm{BCH}$ formula consolidates the series expansion of exponential operator to evaluate such operator evolution.

$$
\exp (-i H t) \sigma(0) \exp (i H t)=\sigma(0)-(i t)[H, \sigma(0)]+\frac{(i t)^{2}}{2 !}[H,[H, \sigma(0)]]-\frac{(i t)^{3}}{3 !}[H,[H,[H, \sigma(0)]]]+\ldots
$$

Now collecting the terms corresponding to the cosine and sine terms, we get the equation for the evolution of $\sigma(0)$. A NMR problem has a well defined, finite dimensional space associated with it and so the number of independent commutators is limited and a series expansion quickly leads to an operator recursion.

Evolution of different initial density operators with some common spin Hamiltonians are given in the table A.2

Table A.2: Time evolution of common product operators for a two spin systems under different spin hamiltonians

\begin{tabular}{|c|c|}
\hline Hamiltonians & Time evolution \\
\hline $\begin{array}{l}\text { Under pulse rotation of angle } \beta \text { and phase } \varphi \\
\qquad H=I_{x} \cos \varphi+I_{y} \sin \varphi\end{array}$ & $\begin{array}{c}I_{z} \stackrel{I_{x} \cos \varphi+I_{y} \sin \varphi}{\longrightarrow} I_{z} \cos \beta+I_{x} \sin \beta \sin \varphi-I_{y} \sin \beta \cos \varphi \\
I_{x} \stackrel{I_{x} \cos \varphi+I_{y} \sin \varphi}{\longrightarrow}-I_{z} \sin \beta \sin \varphi+I_{x}\left(\cos \beta \sin ^{2} \varphi+\cos ^{2} \varphi\right)+I_{y} \sin ^{2}\left(\frac{\beta}{2}\right) \sin 2 \varphi \\
I_{y} \stackrel{I_{x} \cos \stackrel{\varphi+I_{y}}{\longrightarrow} \sin \varphi}{\longrightarrow} I_{z} \sin \beta \cos \varphi+I_{x} \sin ^{2}\left(\frac{\beta}{2}\right) \sin 2 \varphi+I_{y}\left(\cos \beta \cos ^{2} \varphi+\sin ^{2} \varphi\right)\end{array}$ \\
\hline $\begin{array}{l}\text { Under chemical shift } \\
\qquad \Omega t I_{z}\end{array}$ & $\begin{array}{c}I_{x} \stackrel{\Omega t I_{z}}{\longrightarrow} I_{x} \cos \Omega t+I_{y} \sin \Omega t \\
I_{y} \stackrel{\Omega t I_{z}}{\longrightarrow} I_{y} \cos \Omega t-I_{x} \sin \Omega t \\
I_{x} S_{z} \stackrel{\Omega t I_{z}}{\longrightarrow} I_{x} S_{z} \cos \Omega t+I_{y} S_{z} \sin \Omega t\end{array}$ \\
\hline
\end{tabular}


Table A.2 - continued from previous page

\begin{tabular}{|c|c|}
\hline Hamiltonians & Time evolution \\
\hline & $I^{ \pm} \stackrel{\Omega t I_{z}}{\longrightarrow} I^{ \pm} e^{\mp \Omega t}$ \\
\hline & $I_{x} \stackrel{\pi J_{I S t 2 I_{z} S_{z}}}{\longrightarrow} I_{x} \cos \left(\pi J_{I S} t\right)+2 I_{y} S_{z} \sin \left(\pi J_{I S} t\right)$ \\
\hline Under weak coupling $J_{I S}$ & $I_{y} \stackrel{\pi J_{I S} t 2 I_{z} S_{z}}{\longrightarrow} I_{y} \cos \left(\pi J_{I S} t\right)-2 I_{x} S_{z} \sin \left(\pi J_{I S} t\right)$ \\
\hline \multirow[t]{2}{*}{$\pi J_{I S} t 2 I_{z} S_{z}$} & $2 I_{x} S_{z} \stackrel{\pi J_{I S} t 2 I_{z} S_{z}}{\longrightarrow} 2 I_{x} S_{z} \cos \left(\pi J_{I S} t\right)+I_{y} \sin \left(\pi J_{I S} t\right)$ \\
\hline & $2 I_{y} S_{z} \stackrel{\pi J_{I S} t 2 I_{z} S_{z}}{\longrightarrow} 2 I_{y} S_{z} \cos \left(\pi J_{I S} t\right)-I_{x} \sin \left(\pi J_{I S} t\right)$ \\
\hline
\end{tabular}




\section{Appendix B}

\section{Chemical shift assignments and residual dipolar couplings.}

In the following sectin, the backbone assignments of CitAP with and without citrate are given. These chemical shifts were not deposited in the BMRB.

Residual dipolar couplings for different proteins measured during this thesis are also summarized.

\section{B.1 Chemical shift Assignments}

Table B.1: Backbone chemical shifts of citrate free CitAP at 310K.

\begin{tabular}{|c|c|c|c|c|c|c|}
\hline \hline Residue No. & Residue name & $\mathbf{C}^{\prime}$ & $\mathbf{C}_{\alpha}$ & $\mathbf{C}_{\beta}$ & $\mathbf{H}^{N}$ & $\mathbf{N}$ \\
\hline 1 & MET & 175.4 & 55.41 & 32.97 & 8.46 & 121.5 \\
2 & ASP & 176.3 & 54.42 & 41.32 & 8.355 & 122.1 \\
3 & ILE & 176.2 & 61.67 & 38.62 & 8.109 & 120.8 \\
4 & THR & 175.3 & 62.98 & 69.36 & 7.961 & 116.7 \\
5 & GLU & 177.2 & 58.35 & 29.98 & 8.359 & 122.4 \\
6 & GLU & 178 & 58.54 & 29.73 & 8.329 & 120.1 \\
7 & ARG & 177.7 & 58.21 & 30.1 & 8.023 & 119.9 \\
8 & LEU & 178.2 & 57.21 & 41.88 & 8.058 & 121.1 \\
9 & HIS & 177 & 58.04 & 29.73 & 8.299 & 117 \\
13 & GLY & - & 47.82 & - & 8.095 & 107.7 \\
14 & GLN & - & 58.47 & 28.65 & 7.774 & 118.9 \\
18 & ILE & 178.8 & 65.14 & 37.5 & 8.329 & 118.5 \\
19 & GLN & 177.8 & 59.37 & 29.59 & 7.793 & 118.3 \\
20 & ALA & 180.1 & 55.85 & 17.21 & 8.322 & 121.6 \\
21 & MET & 178.9 & 59.32 & 33.56 & 8.607 & 117.4 \\
22 & GLN & 179.1 & 59.09 & 28.55 & 8.282 & 119.4 \\
23 & ILE & 177.5 & 65.5 & 38.69 & 8.391 & 119.3 \\
24 & SER & 174.5 & 61.15 & 63.39 & 7.998 & 110.3 \\
\hline & \multicolumn{3}{|r|}{ Continued on next page } \\
\hline
\end{tabular}


Table B.1 - continued from previous page

\begin{tabular}{|c|c|c|c|c|c|c|}
\hline Residue No. & Residue name & $\mathrm{C}^{\prime}$ & $\mathbf{C}_{\alpha}$ & $\mathbf{C}_{\beta}$ & $\mathbf{H}^{N}$ & $\mathbf{N}$ \\
\hline 25 & ALA & 178.2 & 51.25 & 19.73 & 6.869 & 120.2 \\
\hline 26 & MET & 175.6 & 55.42 & 33.56 & 7.612 & 122.2 \\
\hline 28 & GLU & 179.1 & 59.57 & 29.97 & 9.71 & 116.4 \\
\hline 29 & LEU & 177.5 & 56.87 & 42.02 & 7.323 & 120 \\
\hline 30 & VAL & 177.7 & 67.58 & 31.86 & 7.719 & 119.8 \\
\hline 31 & GLU & 177.9 & 59.44 & 29.96 & 7.609 & 116.3 \\
\hline 32 & ALA & 179.8 & 55.52 & 19.02 & 7.619 & 120.1 \\
\hline 33 & VAL & 179.4 & 67.45 & 31.05 & 8.622 & 117.9 \\
\hline 34 & GLN & 178.3 & 59.78 & 28.87 & 8.355 & 122.1 \\
\hline 35 & LYS & 175.1 & 56.5 & 32.91 & 7.8 & 115 \\
\hline 36 & ARG & 174.2 & 57.58 & 28.18 & 7.966 & 120.8 \\
\hline 37 & ASP & 175.9 & 52.32 & 40.16 & 8.423 & 118.1 \\
\hline 38 & LEU & 179.9 & 59.04 & 40.35 & 7.964 & 123.4 \\
\hline 39 & ALA & 180.5 & 54.98 & 18.07 & 8.355 & 122.1 \\
\hline 40 & ARG & 178 & 58.03 & 30.09 & 7.728 & 121.3 \\
\hline 41 & ILE & 176.8 & 66.78 & 38.22 & 7.961 & 117.6 \\
\hline 42 & LYS & 177.1 & 59.85 & 32.34 & 7.666 & 119.2 \\
\hline 43 & ALA & 179.8 & 54.07 & 18.36 & 7.646 & 118.3 \\
\hline 44 & LEU & 178 & 56.64 & 43.44 & 7.66 & 116.2 \\
\hline 60 & GLY & 172.7 & 43.94 & - & 9.592 & 117.3 \\
\hline 61 & ASP & 177.3 & 52.59 & 41.07 & 8.471 & 118.8 \\
\hline 62 & ALA & 177.2 & 54.17 & 18.37 & 7.449 & 118.7 \\
\hline 63 & SER & 177.2 & 57.91 & 64.36 & 8.32 & 112.8 \\
\hline 81 & GLY & 175.1 & 45.38 & - & 8.443 & 110.2 \\
\hline 82 & GLY & 173.8 & 45.71 & & 8.113 & 108.3 \\
\hline 83 & ASP & 176.5 & 54.22 & 40.75 & 8.273 & 119.8 \\
\hline 112 & ILE & 175.3 & 60.62 & 39.58 & 8.147 & 120.4 \\
\hline 113 & GLN & 175.3 & 53.83 & 34.06 & 9.129 & 127.4 \\
\hline 114 & ASP & 177.9 & 51.99 & 40.93 & 8.429 & 119.7 \\
\hline 115 & ALA & 179 & 55.01 & 18.76 & 8.442 & 119.3 \\
\hline 116 & THR & 175.3 & 61.81 & 70.18 & 8.473 & 108.2 \\
\hline 117 & GLY & 173.8 & 44.66 & - & 8.124 & 110.2 \\
\hline 118 & LYS & 176.2 & 56.44 & 32.8 & 7.658 & 124 \\
\hline 119 & VAL & 176.8 & 63.92 & 31.46 & 8.707 & 125.4 \\
\hline 120 & ILE & 174.6 & 60.7 & 39.65 & 8.985 & 120.4 \\
\hline 121 & GLY & 170.7 & 45.99 & - & 7.697 & 107.5 \\
\hline 122 & ILE & 173 & 59.61 & 41.97 & 8.661 & 118.3 \\
\hline 123 & VAL & 174.1 & 61.11 & 34.18 & 8.812 & 126.5 \\
\hline 124 & SER & - & 55.84 & 65.04 & 8.812 & 120.8 \\
\hline 130 & GLU & 175.8 & 56.79 & 30.54 & 8.587 & 124.9 \\
\hline 131 & GLN & 175.2 & 55.6 & 29.91 & 8.296 & 120.9 \\
\hline 132 & LEU & 176.2 & 55.37 & 42.6 & 8.289 & 124.8 \\
\hline
\end{tabular}


Table B.1 - continued from previous page

\begin{tabular}{|c|c|c|c|c|c|c|}
\hline Residue No. & Residue name & $\mathbf{C}^{\prime}$ & $\mathbf{C}_{\alpha}$ & $\mathbf{C}_{\beta}$ & $\mathbf{H}^{N}$ & $\mathbf{N}$ \\
\hline 133 & GLU & 180.8 & 57.87 & 31.32 & 7.835 & 125.9 \\
\hline \hline
\end{tabular}

Table B.2: Backbone chemical shifts of citrate bound CitAP at 310K.

\begin{tabular}{|c|c|c|c|c|c|c|}
\hline Residue No. & Residue name & $\mathrm{C}^{\prime}$ & $\mathbf{C}_{\alpha}$ & $\mathbf{C}_{\beta}$ & $\mathbf{H}^{N}$ & $\mathbf{N}$ \\
\hline 1 & MET & 175.4 & 55.45 & 32.98 & 8.474 & 121.7 \\
\hline 2 & ASP & 176.3 & 54.31 & 41.45 & 8.349 & 122 \\
\hline 3 & ILE & 176.3 & 61.63 & 38.62 & 8.137 & 120.7 \\
\hline 4 & THR & 175.4 & 63.01 & 69.69 & 8.003 & 116.5 \\
\hline 5 & GLU & 177.3 & 58.13 & 29.99 & 8.327 & 122.3 \\
\hline 6 & GLU & 177.9 & 58.51 & 29.68 & 8.381 & 120.7 \\
\hline 7 & $\mathrm{ARG}$ & 177.9 & 57.78 & 30.15 & 8.084 & 119.7 \\
\hline 8 & LEU & 178.1 & 56.95 & 41.99 & 8.012 & 120.9 \\
\hline 9 & HIS & 177 & 57.4 & 29.4 & 8.266 & 117 \\
\hline 13 & GLY & 174.8 & 65.61 & - & 8.043 & 107.4 \\
\hline 14 & GLN & 178.1 & 63.96 & 28.51 & 7.69 & 118.2 \\
\hline 18 & ILE & 178.7 & 65 & - & 8.307 & 118.3 \\
\hline 20 & ALA & 178.6 & 55.9 & - & 8.555 & 124.7 \\
\hline 21 & MET & 178.8 & 59.18 & 33.54 & 8.633 & 117.3 \\
\hline 25 & ALA & 178.1 & 51.11 & 19.79 & 6.797 & 120.6 \\
\hline 26 & MET & - & 55.25 & 33.36 & 7.639 & 122.6 \\
\hline 29 & LEU & 177.4 & 56.75 & 41.98 & 7.297 & 120 \\
\hline 30 & VAL & 177.7 & 67.19 & 31.9 & 7.77 & 119.9 \\
\hline 31 & GLU & 178 & 59.11 & 29.96 & 7.636 & 116.2 \\
\hline 32 & ALA & 179.7 & 55.32 & 19.18 & 7.618 & 120.1 \\
\hline 33 & VAL & 179.4 & 67.19 & 31.08 & 8.634 & 118 \\
\hline 34 & GLN & 178.4 & 59.71 & 28.89 & 8.349 & 122 \\
\hline 35 & LYS & 175 & 56.19 & 33 & 7.741 & 115 \\
\hline 36 & ARG & 174 & 57.48 & 28.41 & 7.988 & 121 \\
\hline 37 & ASP & 175.9 & 52.16 & 40.45 & 8.3 & 117.8 \\
\hline 38 & LEU & 180 & 59.22 & 40.59 & 8.035 & 123.5 \\
\hline 39 & ALA & 180.6 & 54.95 & 18.17 & 8.404 & 122.3 \\
\hline 40 & $\mathrm{ARG}$ & 178.2 & 57.73 & 30.14 & 7.842 & 121.8 \\
\hline 41 & ILE & 176.9 & 66.6 & 38.38 & 8.086 & 117.8 \\
\hline 42 & LYS & 177 & 60.18 & 32.53 & 7.87 & 119.9 \\
\hline 43 & ALA & 179.7 & 54.08 & 18.47 & 7.767 & 118.4 \\
\hline 44 & LEU & 178.2 & 56.21 & 43.6 & 7.657 & 116.4 \\
\hline 45 & ILE & 177.8 & 60.42 & 35.49 & 8.063 & 116.9 \\
\hline 56 & TYR & 172.2 & 56.39 & 40.2 & 7.325 & 113.4 \\
\hline 57 & ILE & 176.5 & 61.3 & 42.85 & 6.075 & 116.1 \\
\hline
\end{tabular}


Table B.2 - continued from previous page

\begin{tabular}{|c|c|c|c|c|c|c|}
\hline Residue No. & Residue name & $\mathrm{C}^{\prime}$ & $\mathbf{C}_{\alpha}$ & $\mathbf{C}_{\beta}$ & $\mathbf{H}^{N}$ & $\mathbf{N}$ \\
\hline 58 & THR & 173.6 & 61.88 & 72.54 & 10.26 & 127.9 \\
\hline 59 & VAL & 175 & 60.87 & 34.31 & 8.174 & 123.7 \\
\hline 60 & GLY & 172.5 & 43.9 & -- & 9.713 & 118 \\
\hline 61 & ASP & 177.2 & 52.36 & 40.96 & 8.616 & 119 \\
\hline 62 & ALA & 176.9 & 54.61 & 18.56 & 7.317 & 117.9 \\
\hline 63 & SER & 175.4 & 57.38 & 64.77 & 8.264 & 111.6 \\
\hline 64 & GLY & 172.8 & 45.64 & - & 8.264 & 111.6 \\
\hline 65 & GLN & 177.2 & 55.28 & 29.52 & 8.361 & 120.9 \\
\hline 66 & ARG & 177 & 59.13 & 30.83 & 8.998 & 121.8 \\
\hline 67 & LEU & 175 & 54.5 & 43.93 & 9.771 & 125.4 \\
\hline 68 & TYR & 172.7 & 59.94 & 42.21 & 8.02 & 115.9 \\
\hline 69 & HIS & 172.8 & 55.21 & 35.06 & 6.225 & 127.3 \\
\hline 70 & VAL & 175.7 & 64.23 & 31.21 & 7.909 & 118.9 \\
\hline 73 & ASP & 176.7 & 55.79 & 40.34 & 7.914 & 116.3 \\
\hline 74 & GLU & 178.3 & 55.18 & 27.93 & 7.914 & 116.3 \\
\hline 75 & ILE & 176.4 & 64.66 & 37.22 & 7.09 & 122.1 \\
\hline 76 & GLY & 173.4 & 44.92 & - & 8.786 & 114.9 \\
\hline 77 & LYS & 176 & 54.49 & 34.67 & 7.114 & 118.5 \\
\hline 78 & SER & 174.2 & 59.57 & 63.86 & 8.466 & 116.4 \\
\hline 81 & GLY & 175.8 & 45.11 & - & 8.584 & 110.4 \\
\hline 82 & GLY & 175.2 & 46.71 & - & 8.884 & 108.5 \\
\hline 83 & ASP & 178.3 & 52.45 & 39.98 & 8.363 & 117 \\
\hline 84 & SER & 173.4 & 60.92 & 64.31 & 8.896 & 115.9 \\
\hline 85 & $\mathrm{ASP}$ & 177.9 & 58.19 & 40.32 & 8.103 & 122.9 \\
\hline 86 & GLU & 178.6 & 59.89 & 28.32 & 8.935 & 119.3 \\
\hline 87 & ALA & 178 & 54.8 & 18.77 & 7.341 & 122.2 \\
\hline 88 & LEU & 178 & 57.32 & 42.8 & 8.075 & 114.6 \\
\hline 92 & LYS & 174.6 & 55.86 & 34.63 & 8.029 & 120.3 \\
\hline 93 & SER & 174.8 & 57.66 & 65.44 & 7.96 & 112.3 \\
\hline 94 & TYR & 172.2 & 56.45 & 38.53 & 7.901 & 120.7 \\
\hline 95 & VAL & 176.1 & 60.21 & 33.88 & 8.339 & 117.5 \\
\hline 96 & SER & 172.7 & 56.31 & 64.87 & 9.304 & 121.2 \\
\hline 97 & VAL & - & 61.17 & 33.69 & 8.242 & 127.1 \\
\hline 103 & GLY & - & 43.26 & - & 7.253 & 122.6 \\
\hline 107 & ARG & 175.4 & 56.17 & 32.73 & 8.218 & 120.2 \\
\hline 108 & GLY & 171.4 & 44.22 & - & 7.251 & 114.2 \\
\hline 109 & LYS & 175 & 53.88 & 34.26 & 8.59 & 121.7 \\
\hline 110 & SER & 172.5 & 54.28 & 66.53 & 8.178 & 111.5 \\
\hline 112 & ILE & 175.5 & 60.49 & 39.66 & 7.967 & 119.8 \\
\hline 113 & GLN & 175.2 & 53.74 & 34.67 & 9.267 & 127.7 \\
\hline 114 & ASP & 178 & 51.84 & 40.94 & 8.439 & 119.6 \\
\hline 115 & ALA & 179.1 & 54.78 & 18.75 & 8.436 & 119.3 \\
\hline
\end{tabular}


Table B.2 - continued from previous page

\begin{tabular}{|c|c|c|c|c|c|c|}
\hline Residue No. & Residue name & $\mathbf{C}$ & $\mathbf{C}_{\alpha}$ & $\mathbf{C}_{\beta}$ & $\mathbf{H}^{N}$ & $\mathbf{N}$ \\
\hline 116 & THR & 175.3 & 61.59 & 70.11 & 8.488 & 108.3 \\
117 & GLY & 174 & 44.63 & - & 8.136 & 110.3 \\
118 & LYS & 176.3 & 56.37 & 32.89 & 7.646 & 124.5 \\
119 & VAL & 176.9 & 64.36 & 31.78 & 8.828 & 125.6 \\
120 & ILE & 174.3 & 60.46 & 39.96 & 9.04 & 120 \\
121 & GLY & 170.7 & 46.14 & - & 7.666 & 107.7 \\
122 & ILE & 171.2 & 59.65 & 41.51 & 8.822 & 119.2 \\
123 & VAL & 173.2 & 61.16 & 34.5 & 8.73 & 126 \\
124 & SER & 174 & 54.65 & 66.18 & - & - \\
125 & VAL & 174.7 & 61.52 & 33.1 & 9.135 & 128.8 \\
126 & GLY & 171.9 & 44.28 & - & 7.18 & 111.9 \\
127 & TYR & 176.1 & 56.38 & 41.7 & 8.968 & 118.6 \\
128 & THR & 175.2 & 62.24 & 68.72 & 8.633 & 115 \\
129 & ILE & 176.7 & 61.56 & 38.81 & 7.839 & 121 \\
130 & GLU & 176.6 & 57.59 & 29.93 & 8.474 & 121.7 \\
131 & GLN & 175.7 & 56.05 & 29.53 & 8.003 & 119 \\
132 & LEU & 176.2 & 55.17 & 42.62 & 7.979 & 122.4 \\
133 & GLU & 180.8 & 57.88 & 31.36 & 7.71 & 125.4 \\
\hline \hline
\end{tabular}




\section{B.2 Residual dipolar coupling}

Table B.3: Residual dipolar couplings measured for DcuS-PD in phages

\begin{tabular}{|c|c|c|c|c|c|c|}
\hline Res: Number1 & Res: name1 & Atom1 & Res: Number2 & Res: name2 & Atom2 & $\mathbf{D}$ \\
\hline 24 & MET & $\mathrm{H}$ & 24 & MET & $\mathrm{N}$ & -7.272 \\
\hline 25 & THR & $\mathrm{H}$ & 25 & THR & $\mathrm{N}$ & 6.874 \\
\hline 26 & ARG & $\mathrm{H}$ & 26 & ARG & $\mathrm{N}$ & -5.494 \\
\hline 27 & ASP & $\mathrm{H}$ & 27 & ASP & $\mathrm{N}$ & -7.022 \\
\hline 28 & GLY & $\mathrm{H}$ & 28 & GLY & $\mathrm{N}$ & -5.500 \\
\hline 29 & LEU & $\mathrm{H}$ & 29 & LEU & $\mathrm{N}$ & -2.338 \\
\hline 30 & ALA & $\mathrm{H}$ & 30 & ALA & $\mathrm{N}$ & -9.714 \\
\hline 31 & $\mathrm{APN}$ & $\mathrm{H}$ & 31 & APN & $\mathrm{N}$ & -16.478 \\
\hline 32 & LYS & $\mathrm{H}$ & 32 & LYS & $\mathrm{N}$ & -8.552 \\
\hline 33 & ALA & $\mathrm{H}$ & 33 & ALA & $\mathrm{N}$ & 6.582 \\
\hline 34 & LEU & $\mathrm{H}$ & 34 & LEU & $\mathrm{N}$ & -11.398 \\
\hline 35 & ALA & $\mathrm{H}$ & 35 & ALA & $\mathrm{N}$ & -19.018 \\
\hline 36 & VAL & $\mathrm{H}$ & 36 & VAL & $\mathrm{N}$ & -7.022 \\
\hline 37 & ALA & $\mathrm{H}$ & 37 & ALA & $\mathrm{N}$ & -13.298 \\
\hline 38 & ARG & $\mathrm{H}$ & 38 & ARG & $\mathrm{N}$ & -21.694 \\
\hline 39 & THR & $\mathrm{H}$ & 39 & THR & $\mathrm{N}$ & -15.794 \\
\hline 40 & LEU & $\mathrm{H}$ & 40 & LEU & $\mathrm{N}$ & -9.366 \\
\hline 41 & ALA & $\mathrm{H}$ & 41 & ALA & $\mathrm{N}$ & -19.112 \\
\hline 42 & ASP & $\mathrm{H}$ & 42 & ASP & $\mathrm{N}$ & -21.046 \\
\hline 46 & ILE & $\mathrm{H}$ & 46 & ILE & $\mathrm{N}$ & 7.626 \\
\hline 47 & ARG & $\mathrm{H}$ & 47 & ARG & $\mathrm{N}$ & 8.266 \\
\hline 48 & GLN & $\mathrm{H}$ & 48 & GLN & $\mathrm{N}$ & 2.606 \\
\hline 49 & GLY & $\mathrm{H}$ & 49 & GLY & $\mathrm{N}$ & -5.478 \\
\hline 50 & LEU & $\mathrm{H}$ & 50 & LEU & $\mathrm{N}$ & 8.634 \\
\hline 51 & GLN & $\mathrm{H}$ & 51 & GLN & $\mathrm{N}$ & 12.094 \\
\hline 52 & LYS & $\mathrm{H}$ & 52 & LYS & $\mathrm{N}$ & -15.724 \\
\hline 53 & LYS & $\mathrm{H}$ & 53 & LYS & $\mathrm{N}$ & -5.336 \\
\hline 56 & ELU & $\mathrm{H}$ & 56 & ELU & $\mathrm{N}$ & 1.194 \\
\hline 57 & SER & $\mathrm{H}$ & 57 & SER & $\mathrm{N}$ & 14.250 \\
\hline 60 & GLN & $\mathrm{H}$ & 60 & GLN & $\mathrm{N}$ & -10.462 \\
\hline 61 & ALA & $\mathrm{H}$ & 61 & ALA & $\mathrm{N}$ & -17.306 \\
\hline 62 & ILE & $\mathrm{H}$ & 62 & ILE & $\mathrm{N}$ & -7.982 \\
\hline 63 & ALA & $\mathrm{H}$ & 63 & ALA & $\mathrm{N}$ & -10.458 \\
\hline 64 & GLU & $\mathrm{H}$ & 64 & GLU & $\mathrm{N}$ & -18.378 \\
\hline 65 & ALA & $\mathrm{H}$ & 65 & ALA & $\mathrm{N}$ & -18.870 \\
\hline 66 & VAL & $\mathrm{H}$ & 66 & VAL & $\mathrm{N}$ & -15.392 \\
\hline 67 & $\mathrm{ARG}$ & $\mathrm{H}$ & 67 & ARG & $\mathrm{N}$ & -16.418 \\
\hline 68 & LYS & $\mathrm{H}$ & 68 & LYS & $\mathrm{N}$ & -16.696 \\
\hline 70 & $\mathrm{APN}$ & $\mathrm{H}$ & 70 & $\mathrm{APN}$ & $\mathrm{N}$ & -12.502 \\
\hline
\end{tabular}


Table B.3 - continued from previous page

\begin{tabular}{|c|c|c|c|c|c|c|}
\hline Res: Number1 & Res: name1 & Atom1 & Res: Number2 & Res: name2 & Atom2 & $\mathbf{D}$ \\
\hline 71 & ASP & $\mathrm{H}$ & 71 & $\mathrm{ASP}$ & $\mathrm{N}$ & -5.140 \\
\hline 72 & LEU & $\mathrm{H}$ & 72 & LEU & $\mathrm{N}$ & -3.780 \\
\hline 74 & PHE & $\mathrm{H}$ & 74 & PHE & $\mathrm{N}$ & -0.572 \\
\hline 76 & VAL & $\mathrm{H}$ & 76 & VAL & $\mathrm{N}$ & -7.428 \\
\hline 77 & VAL & $\mathrm{H}$ & 77 & VAL & $\mathrm{N}$ & 2.332 \\
\hline 78 & THR & $\mathrm{H}$ & 78 & THR & $\mathrm{N}$ & 2.972 \\
\hline 79 & ASP & $\mathrm{H}$ & 79 & ASP & $\mathrm{N}$ & 14.192 \\
\hline 80 & MET & $\mathrm{H}$ & 80 & MET & $\mathrm{N}$ & -14.188 \\
\hline 81 & GLN & $\mathrm{H}$ & 81 & GLN & $\mathrm{N}$ & 3.190 \\
\hline 82 & SER & $\mathrm{H}$ & 82 & SER & $\mathrm{N}$ & 15.838 \\
\hline 83 & LEU & $\mathrm{H}$ & 83 & LEU & $\mathrm{N}$ & -5.574 \\
\hline 84 & ARG & $\mathrm{H}$ & 84 & ARG & $\mathrm{N}$ & 19.856 \\
\hline 85 & TYR & $\mathrm{H}$ & 85 & TYR & $\mathrm{N}$ & -11.966 \\
\hline 87 & HIS & $\mathrm{H}$ & 87 & HIS & $\mathrm{N}$ & 6.322 \\
\hline 90 & ALA & $\mathrm{H}$ & 90 & ALA & $\mathrm{N}$ & -5.450 \\
\hline 91 & GLN & $\mathrm{H}$ & 91 & GLN & $\mathrm{N}$ & -12.010 \\
\hline 92 & ARG & $\mathrm{H}$ & 92 & ARG & $\mathrm{N}$ & -3.392 \\
\hline 93 & ILE & $\mathrm{H}$ & 93 & ILE & $\mathrm{N}$ & -2.746 \\
\hline 94 & GLY & $\mathrm{H}$ & 94 & GLY & $\mathrm{N}$ & 8.126 \\
\hline 95 & GLN & $\mathrm{H}$ & 95 & GLN & $\mathrm{N}$ & -3.454 \\
\hline 97 & PHE & $\mathrm{H}$ & 97 & PHE & $\mathrm{N}$ & -1.800 \\
\hline 98 & LYS & $\mathrm{H}$ & 98 & LYS & $\mathrm{N}$ & 1.610 \\
\hline 99 & GLY & $\mathrm{H}$ & 99 & GLY & $\mathrm{N}$ & -1.602 \\
\hline 101 & ASP & $\mathrm{H}$ & 101 & ASP & $\mathrm{N}$ & -4.604 \\
\hline 102 & ILE & $\mathrm{H}$ & 102 & ILE & $\mathrm{N}$ & 12.356 \\
\hline 103 & LEU & $\mathrm{H}$ & 103 & LEU & $\mathrm{N}$ & 2.232 \\
\hline 104 & LYS & $\mathrm{H}$ & 104 & LYS & $\mathrm{N}$ & -7.958 \\
\hline 105 & ALA & $\mathrm{H}$ & 105 & ALA & $\mathrm{N}$ & 6.346 \\
\hline 106 & LEU & $\mathrm{H}$ & 106 & LEU & $\mathrm{N}$ & 2.624 \\
\hline 108 & GLY & $\mathrm{H}$ & 108 & GLY & $\mathrm{N}$ & -1.972 \\
\hline 109 & GLU & $\mathrm{H}$ & 109 & GLU & $\mathrm{N}$ & 15.184 \\
\hline 110 & GLU & $\mathrm{H}$ & 110 & GLU & $\mathrm{N}$ & 5.218 \\
\hline 112 & VAL & $\mathrm{H}$ & 112 & VAL & $\mathrm{N}$ & 3.264 \\
\hline 113 & ALA & $\mathrm{H}$ & 113 & ALA & $\mathrm{N}$ & 4.866 \\
\hline 114 & ILE & $\mathrm{H}$ & 114 & ILE & $\mathrm{N}$ & 6.614 \\
\hline 115 & APN & $\mathrm{H}$ & 115 & $\mathrm{APN}$ & $\mathrm{N}$ & 9.890 \\
\hline 116 & ARG & $\mathrm{H}$ & 116 & ARG & $\mathrm{N}$ & -8.614 \\
\hline 121 & GLN & $\mathrm{H}$ & 121 & GLN & $\mathrm{N}$ & -1.332 \\
\hline 122 & ALA & $\mathrm{H}$ & 122 & ALA & $\mathrm{N}$ & 0.396 \\
\hline 123 & LEU & $\mathrm{H}$ & 123 & LEU & $\mathrm{N}$ & 9.168 \\
\hline 125 & VAL & $\mathrm{H}$ & 125 & VAL & $\mathrm{N}$ & -1.902 \\
\hline 126 & PHE & $\mathrm{H}$ & 126 & PHE & $\mathrm{N}$ & -2.088 \\
\hline \multicolumn{7}{|c|}{ Continued on next page } \\
\hline
\end{tabular}


Table B.3 - continued from previous page

\begin{tabular}{|c|c|c|c|c|c|c|}
\hline Res: Number1 & Res: name1 & Atom1 & Res: Number2 & Res: name2 & Atom2 & $\mathbf{D}$ \\
\hline 127 & THR & $\mathrm{H}$ & 127 & THR & $\mathrm{N}$ & 2.014 \\
\hline 129 & ILE & $\mathrm{H}$ & 129 & ILE & $\mathrm{N}$ & 16.536 \\
\hline 130 & TYR & $\mathrm{H}$ & 130 & TYR & $\mathrm{N}$ & 3.082 \\
\hline 131 & ASP & $\mathrm{H}$ & 131 & ASP & $\mathrm{N}$ & 10.472 \\
\hline 132 & GLU & $\mathrm{H}$ & 132 & GLU & $\mathrm{N}$ & -2.890 \\
\hline 133 & APN & $\mathrm{H}$ & 133 & $\mathrm{APN}$ & $\mathrm{N}$ & 13.834 \\
\hline 134 & HIS & $\mathrm{H}$ & 134 & HIS & $\mathrm{N}$ & 2.788 \\
\hline 136 & GLN & $\mathrm{H}$ & 136 & GLN & $\mathrm{N}$ & 3.522 \\
\hline 137 & ILE & $\mathrm{H}$ & 137 & ILE & $\mathrm{N}$ & 0.682 \\
\hline 138 & GLY & $\mathrm{H}$ & 138 & GLY & $\mathrm{N}$ & 2.878 \\
\hline 139 & VAL & $\mathrm{H}$ & 139 & VAL & $\mathrm{N}$ & -0.630 \\
\hline 140 & VAL & $\mathrm{H}$ & 140 & VAL & $\mathrm{N}$ & -0.220 \\
\hline 141 & ALA & $\mathrm{H}$ & 141 & ALA & $\mathrm{N}$ & -5.122 \\
\hline 144 & LEU & $\mathrm{H}$ & 144 & LEU & $\mathrm{N}$ & 1.302 \\
\hline 145 & GLU & $\mathrm{H}$ & 145 & GLU & $\mathrm{N}$ & 10.844 \\
\hline 146 & LEU & $\mathrm{H}$ & 146 & LEU & $\mathrm{N}$ & 7.608 \\
\hline 148 & ARG & $\mathrm{H}$ & 148 & ARG & $\mathrm{N}$ & -17.148 \\
\hline 149 & VAL & $\mathrm{H}$ & 149 & VAL & $\mathrm{N}$ & 6.244 \\
\hline 150 & THR & $\mathrm{H}$ & 150 & THR & $\mathrm{N}$ & -1.920 \\
\hline 151 & GLN & $\mathrm{H}$ & 151 & GLN & $\mathrm{N}$ & -4.052 \\
\hline 152 & GLN & $\mathrm{H}$ & 152 & GLN & $\mathrm{N}$ & -3.960 \\
\hline 153 & ILE & $\mathrm{H}$ & 153 & ILE & $\mathrm{N}$ & 2.510 \\
\hline 154 & APN & $\mathrm{H}$ & 154 & APN & $\mathrm{N}$ & -0.164 \\
\hline 155 & ASP & $\mathrm{H}$ & 155 & ASP & $\mathrm{N}$ & -2.700 \\
\hline 156 & SER & $\mathrm{H}$ & 156 & SER & $\mathrm{N}$ & 0.432 \\
\hline 157 & ARG & $\mathrm{H}$ & 157 & ARG & $\mathrm{N}$ & 0.444 \\
\hline 23 & ASP & $\mathrm{C}$ & 24 & MET & $\mathrm{N}$ & -0.622 \\
\hline 24 & MET & $\mathrm{C}$ & 25 & THR & $\mathrm{N}$ & -0.382 \\
\hline 25 & THR & $\mathrm{C}$ & 26 & ARG & $\mathrm{N}$ & 1.683 \\
\hline 26 & ARG & $\mathrm{C}$ & 27 & ASP & $\mathrm{N}$ & 0.225 \\
\hline 27 & ASP & $\mathrm{C}$ & 28 & GLY & $\mathrm{N}$ & -0.765 \\
\hline 28 & GLY & $\mathrm{C}$ & 29 & LEU & $\mathrm{N}$ & 1.606 \\
\hline 29 & LEU & $\mathrm{C}$ & 30 & ALA & $\mathrm{N}$ & 0.628 \\
\hline 30 & ALA & $\mathrm{C}$ & 31 & $\mathrm{APN}$ & $\mathrm{N}$ & 0.739 \\
\hline 31 & APN & $\mathrm{C}$ & 32 & LYS & $\mathrm{N}$ & -1.178 \\
\hline 32 & LYS & $\mathrm{C}$ & 33 & ALA & $\mathrm{N}$ & 0.647 \\
\hline 33 & ALA & $\mathrm{C}$ & 34 & LEU & $\mathrm{N}$ & 0.132 \\
\hline 34 & LEU & $\mathrm{C}$ & 35 & ALA & $\mathrm{N}$ & 0.282 \\
\hline 35 & ALA & $\mathrm{C}$ & 36 & VAL & $\mathrm{N}$ & 0.273 \\
\hline 36 & VAL & $\mathrm{C}$ & 37 & ALA & $\mathrm{N}$ & 1.602 \\
\hline 37 & ALA & $\mathrm{C}$ & 38 & ARG & $\mathrm{N}$ & 0.292 \\
\hline 38 & ARG & $\mathrm{C}$ & 39 & THR & $\mathrm{N}$ & -0.614 \\
\hline
\end{tabular}


Table B.3 - continued from previous page

\begin{tabular}{|c|c|c|c|c|c|c|}
\hline Res: Number1 & Res: name1 & Atom1 & Res: Number2 & Res: name2 & Atom2 & $\mathbf{D}$ \\
\hline 39 & THR & $\mathrm{C}$ & 40 & LEU & $\mathrm{N}$ & 1.937 \\
\hline 40 & LEU & $\mathrm{C}$ & 41 & ALA & $\mathrm{N}$ & 0.523 \\
\hline 41 & ALA & $\mathrm{C}$ & 42 & ASP & $\mathrm{N}$ & 1.477 \\
\hline 42 & ASP & $\mathrm{C}$ & 43 & SER & $\mathrm{N}$ & 0.302 \\
\hline 44 & PRO & $\mathrm{C}$ & 45 & GLU & $\mathrm{N}$ & -1.591 \\
\hline 45 & GLU & $\mathrm{C}$ & 46 & ILE & $\mathrm{N}$ & -1.031 \\
\hline 46 & ILE & $\mathrm{C}$ & 47 & ARG & $\mathrm{N}$ & 0.908 \\
\hline 47 & ARG & $\mathrm{C}$ & 48 & GLN & $\mathrm{N}$ & 0.745 \\
\hline 48 & GLN & $\mathrm{C}$ & 49 & GLY & $\mathrm{N}$ & -1.598 \\
\hline 49 & GLY & $\mathrm{C}$ & 50 & LEU & $\mathrm{N}$ & 0.169 \\
\hline 50 & LEU & $\mathrm{C}$ & 51 & GLN & $\mathrm{N}$ & 0.533 \\
\hline 51 & GLN & $\mathrm{C}$ & 52 & LYS & $\mathrm{N}$ & -0.893 \\
\hline 52 & LYS & $\mathrm{C}$ & 53 & LYS & $\mathrm{N}$ & 0.647 \\
\hline 55 & GLN & $\mathrm{C}$ & 56 & ELU & $\mathrm{N}$ & -0.638 \\
\hline 56 & ELU & $\mathrm{C}$ & 57 & SER & $\mathrm{N}$ & -1.319 \\
\hline 57 & SER & $\mathrm{C}$ & 58 & GLY & $\mathrm{N}$ & -0.173 \\
\hline 59 & ILE & $\mathrm{C}$ & 60 & GLN & $\mathrm{N}$ & 1.274 \\
\hline 60 & GLN & $\mathrm{C}$ & 61 & ALA & $\mathrm{N}$ & 0.411 \\
\hline 61 & ALA & $\mathrm{C}$ & 62 & ILE & $\mathrm{N}$ & -0.394 \\
\hline 62 & ILE & $\mathrm{C}$ & 63 & ALA & $\mathrm{N}$ & 1.708 \\
\hline 63 & ALA & $\mathrm{C}$ & 64 & GLU & $\mathrm{N}$ & -0.252 \\
\hline 64 & GLU & $\mathrm{C}$ & 65 & ALA & $\mathrm{N}$ & 1.128 \\
\hline 65 & ALA & $\mathrm{C}$ & 66 & VAL & $\mathrm{N}$ & -0.432 \\
\hline 66 & VAL & $\mathrm{C}$ & 67 & ARG & $\mathrm{N}$ & 5.007 \\
\hline 67 & ARG & $\mathrm{C}$ & 68 & LYS & $\mathrm{N}$ & 0.094 \\
\hline 68 & LYS & $\mathrm{C}$ & 69 & ARG & $\mathrm{N}$ & 0.864 \\
\hline 69 & ARG & $\mathrm{C}$ & 70 & APN & $\mathrm{N}$ & 1.093 \\
\hline 70 & APN & $\mathrm{C}$ & 71 & ASP & $\mathrm{N}$ & 0.385 \\
\hline 71 & ASP & $\mathrm{C}$ & 72 & LEU & $\mathrm{N}$ & -1.628 \\
\hline 72 & LEU & $\mathrm{C}$ & 73 & LEU & $\mathrm{N}$ & 1.268 \\
\hline 73 & LEU & $\mathrm{C}$ & 74 & PHE & $\mathrm{N}$ & -0.606 \\
\hline 75 & ILE & $\mathrm{C}$ & 76 & VAL & $\mathrm{N}$ & 0.123 \\
\hline 76 & VAL & $\mathrm{C}$ & 77 & VAL & $\mathrm{N}$ & -1.327 \\
\hline 77 & VAL & $\mathrm{C}$ & 78 & THR & $\mathrm{N}$ & -0.364 \\
\hline 78 & THR & $\mathrm{C}$ & 79 & ASP & $\mathrm{N}$ & -0.934 \\
\hline 79 & ASP & $\mathrm{C}$ & 80 & MET & $\mathrm{N}$ & -2.152 \\
\hline 80 & MET & $\mathrm{C}$ & 81 & GLN & $\mathrm{N}$ & 0.252 \\
\hline 81 & GLN & $\mathrm{C}$ & 82 & SER & $\mathrm{N}$ & -0.175 \\
\hline 82 & SER & $\mathrm{C}$ & 83 & LEU & $\mathrm{N}$ & -1.468 \\
\hline 83 & LEU & $\mathrm{C}$ & 84 & ARG & $\mathrm{N}$ & -0.553 \\
\hline 84 & $\mathrm{ARG}$ & $\mathrm{C}$ & 85 & TYR & $\mathrm{N}$ & 1.928 \\
\hline 85 & TYR & $\mathrm{C}$ & 86 & SER & $\mathrm{N}$ & -1.422 \\
\hline
\end{tabular}


Table B.3 - continued from previous page

\begin{tabular}{|c|c|c|c|c|c|c|}
\hline Res: Number1 & Res: name1 & Atom1 & Res: Number2 & Res: name2 & Atom2 & $\mathbf{D}$ \\
\hline 86 & SER & $\mathrm{C}$ & 87 & HIS & $\mathrm{N}$ & -0.992 \\
\hline 89 & GLU & $\mathrm{C}$ & 90 & ALA & $\mathrm{N}$ & -0.226 \\
\hline 90 & ALA & $\mathrm{C}$ & 91 & GLN & $\mathrm{N}$ & 1.530 \\
\hline 91 & GLN & $\mathrm{C}$ & 92 & ARG & $\mathrm{N}$ & -0.327 \\
\hline 92 & ARG & $\mathrm{C}$ & 93 & ILE & $\mathrm{N}$ & 1.138 \\
\hline 93 & ILE & $\mathrm{C}$ & 94 & GLY & $\mathrm{N}$ & -0.688 \\
\hline 94 & GLY & $\mathrm{C}$ & 95 & GLN & $\mathrm{N}$ & -1.783 \\
\hline 96 & PRO & $\mathrm{C}$ & 97 & PHE & $\mathrm{N}$ & 0.400 \\
\hline 97 & PHE & $\mathrm{C}$ & 98 & LYS & $\mathrm{N}$ & -2.131 \\
\hline 98 & LYS & $\mathrm{C}$ & 99 & GLY & $\mathrm{N}$ & 0.299 \\
\hline 99 & GLY & $\mathrm{C}$ & 100 & ASP & $\mathrm{N}$ & 0.000 \\
\hline 100 & ASP & $\mathrm{C}$ & 101 & ASP & $\mathrm{N}$ & -0.138 \\
\hline 101 & ASP & $\mathrm{C}$ & 102 & ILE & $\mathrm{N}$ & -1.360 \\
\hline 102 & ILE & $\mathrm{C}$ & 103 & LEU & $\mathrm{N}$ & 1.556 \\
\hline 103 & LEU & $\mathrm{C}$ & 104 & LYS & $\mathrm{N}$ & -1.478 \\
\hline 104 & LYS & $\mathrm{C}$ & 105 & ALA & $\mathrm{N}$ & 0.042 \\
\hline 105 & ALA & $\mathrm{C}$ & 106 & LEU & $\mathrm{N}$ & 1.219 \\
\hline 107 & APN & $\mathrm{C}$ & 108 & GLY & $\mathrm{N}$ & 0.458 \\
\hline 108 & GLY & C & 109 & GLU & $\mathrm{N}$ & -0.550 \\
\hline 109 & GLU & $\mathrm{C}$ & 110 & GLU & $\mathrm{N}$ & -0.978 \\
\hline 111 & APN & $\mathrm{C}$ & 112 & VAL & $\mathrm{N}$ & -1.237 \\
\hline 112 & VAL & $\mathrm{C}$ & 113 & ALA & $\mathrm{N}$ & 0.376 \\
\hline 113 & ALA & $\mathrm{C}$ & 114 & ILE & $\mathrm{N}$ & 0.831 \\
\hline 114 & ILE & $\mathrm{C}$ & 115 & APN & $\mathrm{N}$ & -0.356 \\
\hline 115 & APN & $\mathrm{C}$ & 116 & ARG & $\mathrm{N}$ & 0.576 \\
\hline 116 & ARG & $\mathrm{C}$ & 117 & GLY & $\mathrm{N}$ & 0.686 \\
\hline 120 & ALA & $\mathrm{C}$ & 121 & GLN & $\mathrm{N}$ & -0.590 \\
\hline 121 & GLN & $\mathrm{C}$ & 122 & ALA & $\mathrm{N}$ & -0.735 \\
\hline 122 & ALA & $\mathrm{C}$ & 123 & LEU & $\mathrm{N}$ & 1.189 \\
\hline 124 & ARG & $\mathrm{C}$ & 125 & VAL & $\mathrm{N}$ & 1.133 \\
\hline 125 & VAL & $\mathrm{C}$ & 126 & PHE & $\mathrm{N}$ & -0.352 \\
\hline 126 & PHE & $\mathrm{C}$ & 127 & THR & $\mathrm{N}$ & -0.905 \\
\hline 128 & PRO & $\mathrm{C}$ & 129 & ILE & $\mathrm{N}$ & -1.748 \\
\hline 129 & ILE & $\mathrm{C}$ & 130 & TYR & $\mathrm{N}$ & 1.793 \\
\hline 130 & TYR & $\mathrm{C}$ & 131 & ASP & $\mathrm{N}$ & -2.142 \\
\hline 131 & ASP & $\mathrm{C}$ & 132 & GLU & $\mathrm{N}$ & -0.205 \\
\hline 132 & GLU & $\mathrm{C}$ & 133 & $\mathrm{APN}$ & $\mathrm{N}$ & 1.344 \\
\hline 133 & APN & $\mathrm{C}$ & 134 & HIS & $\mathrm{N}$ & -2.017 \\
\hline 135 & LYS & $\mathrm{C}$ & 136 & GLN & $\mathrm{N}$ & 1.132 \\
\hline 136 & GLN & $\mathrm{C}$ & 137 & ILE & $\mathrm{N}$ & 0.177 \\
\hline 137 & ILE & $\mathrm{C}$ & 138 & GLY & $\mathrm{N}$ & -2.278 \\
\hline 138 & GLY & $\mathrm{C}$ & 139 & VAL & $\mathrm{N}$ & -1.086 \\
\hline
\end{tabular}


Table B.3 - continued from previous page

\begin{tabular}{|c|c|c|c|c|c|c|}
\hline Res: Number1 & Res: name1 & Atom1 & Res: Number2 & Res: name2 & Atom2 & D \\
\hline 139 & VAL & $\mathrm{C}$ & 140 & VAL & $\mathrm{N}$ & -0.730 \\
\hline 140 & VAL & $\mathrm{C}$ & 141 & ALA & $\mathrm{N}$ & 0.568 \\
\hline 143 & GLY & $\mathrm{C}$ & 144 & LEU & $\mathrm{N}$ & -5.000 \\
\hline 144 & LEU & $\mathrm{C}$ & 145 & GLU & $\mathrm{N}$ & -0.540 \\
\hline 145 & GLU & $\mathrm{C}$ & 146 & $\mathrm{LEU}$ & $\mathrm{N}$ & 1.181 \\
\hline 147 & SER & $\mathrm{C}$ & 148 & ARG & $\mathrm{N}$ & -0.060 \\
\hline 148 & ARG & $\mathrm{C}$ & 149 & VAL & $\mathrm{N}$ & -0.015 \\
\hline 149 & VAL & $\mathrm{C}$ & 150 & THR & $\mathrm{N}$ & 1.012 \\
\hline 150 & THR & $\mathrm{C}$ & 151 & GLN & $\mathrm{N}$ & -0.742 \\
\hline 151 & GLN & $\mathrm{C}$ & 152 & GLN & $\mathrm{N}$ & 0.264 \\
\hline 152 & GLN & $\mathrm{C}$ & 153 & ILE & $\mathrm{N}$ & 0.571 \\
\hline 153 & ILE & $\mathrm{C}$ & 154 & APN & $\mathrm{N}$ & -1.519 \\
\hline 154 & APN & $\mathrm{C}$ & 155 & ASP & $\mathrm{N}$ & 1.224 \\
\hline 155 & ASP & $\mathrm{C}$ & 156 & SER & $\mathrm{N}$ & 0.118 \\
\hline 156 & SER & $\mathrm{C}$ & 157 & ARG & $\mathrm{N}$ & 0.261 \\
\hline 23 & ASP & $\mathrm{CA}$ & 23 & ASP & $\mathrm{C}$ & -1.081 \\
\hline 24 & MET & $\mathrm{CA}$ & 24 & MET & $\mathrm{C}$ & 0.277 \\
\hline 25 & THR & $\mathrm{CA}$ & 25 & THR & $\mathrm{C}$ & 0.764 \\
\hline 26 & ARG & $\mathrm{CA}$ & 26 & ARG & $\mathrm{C}$ & 2.235 \\
\hline 27 & ASP & $\mathrm{CA}$ & 27 & ASP & $\mathrm{C}$ & 0.431 \\
\hline 28 & GLY & $\mathrm{CA}$ & 28 & GLY & $\mathrm{C}$ & 0.090 \\
\hline 29 & LEU & $\mathrm{CA}$ & 29 & LEU & $\mathrm{C}$ & -1.835 \\
\hline 30 & ALA & $\mathrm{CA}$ & 30 & ALA & $\mathrm{C}$ & 3.015 \\
\hline 31 & APN & $\mathrm{CA}$ & 31 & APN & $\mathrm{C}$ & -0.675 \\
\hline 32 & LYS & $\mathrm{CA}$ & 32 & LYS & $\mathrm{C}$ & -8.427 \\
\hline 33 & ALA & $\mathrm{CA}$ & 33 & ALA & $\mathrm{C}$ & -4.001 \\
\hline 34 & LEU & $\mathrm{CA}$ & 34 & LEU & $\mathrm{C}$ & 3.442 \\
\hline 35 & ALA & $\mathrm{CA}$ & 35 & ALA & $\mathrm{C}$ & -2.338 \\
\hline 36 & VAL & $\mathrm{CA}$ & 36 & VAL & $\mathrm{C}$ & -0.189 \\
\hline 37 & ALA & $\mathrm{CA}$ & 37 & ALA & $\mathrm{C}$ & 5.921 \\
\hline 38 & ARG & $\mathrm{CA}$ & 38 & ARG & $\mathrm{C}$ & 3.584 \\
\hline 40 & LEU & $\mathrm{CA}$ & 40 & LEU & $\mathrm{C}$ & -2.709 \\
\hline 41 & ALA & $\mathrm{CA}$ & 41 & ALA & $\mathrm{C}$ & 3.417 \\
\hline 42 & ASP & $\mathrm{CA}$ & 42 & ASP & $\mathrm{C}$ & -4.112 \\
\hline 45 & GLU & $\mathrm{CA}$ & 45 & GLU & $\mathrm{C}$ & -1.326 \\
\hline 46 & ILE & $\mathrm{CA}$ & 46 & ILE & $\mathrm{C}$ & 5.741 \\
\hline 47 & $\mathrm{ARG}$ & $\mathrm{CA}$ & 47 & ARG & $\mathrm{C}$ & -3.139 \\
\hline 48 & GLN & $\mathrm{CA}$ & 48 & GLN & $\mathrm{C}$ & 6.411 \\
\hline 49 & GLY & $\mathrm{CA}$ & 49 & GLY & $\mathrm{C}$ & 4.842 \\
\hline 50 & LEU & $\mathrm{CA}$ & 50 & LEU & $\mathrm{C}$ & 2.117 \\
\hline 51 & GLN & $\mathrm{CA}$ & 51 & GLN & $\mathrm{C}$ & -1.069 \\
\hline 52 & LYS & $\mathrm{CA}$ & 52 & LYS & $\mathrm{C}$ & 2.474 \\
\hline
\end{tabular}


Table B.3 - continued from previous page

\begin{tabular}{|c|c|c|c|c|c|c|}
\hline Res: Number1 & Res: name1 & Atom1 & Res: Number2 & Res: name2 & Atom2 & $\mathbf{D}$ \\
\hline 56 & ELU & $\mathrm{CA}$ & 56 & ELU & $\mathrm{C}$ & 2.229 \\
\hline 58 & GLY & $\mathrm{CA}$ & 58 & GLY & $\mathrm{C}$ & -6.173 \\
\hline 59 & ILE & $\mathrm{CA}$ & 59 & ILE & $\mathrm{C}$ & 3.399 \\
\hline 60 & GLN & $\mathrm{CA}$ & 60 & GLN & $\mathrm{C}$ & -4.454 \\
\hline 61 & ALA & $\mathrm{CA}$ & 61 & ALA & $\mathrm{C}$ & 0.686 \\
\hline 62 & ILE & $\mathrm{CA}$ & 62 & ILE & $\mathrm{C}$ & -0.121 \\
\hline 63 & ALA & $\mathrm{CA}$ & 63 & ALA & $\mathrm{C}$ & -3.641 \\
\hline 64 & GLU & $\mathrm{CA}$ & 64 & GLU & $\mathrm{C}$ & 3.267 \\
\hline 65 & ALA & $\mathrm{CA}$ & 65 & ALA & $\mathrm{C}$ & -1.189 \\
\hline 66 & VAL & $\mathrm{CA}$ & 66 & VAL & $\mathrm{C}$ & -4.916 \\
\hline 67 & ARG & $\mathrm{CA}$ & 67 & ARG & $\mathrm{C}$ & -4.333 \\
\hline 68 & LYS & $\mathrm{CA}$ & 68 & LYS & $\mathrm{C}$ & 1.372 \\
\hline 69 & ARG & $\mathrm{CA}$ & 69 & ARG & $\mathrm{C}$ & -1.871 \\
\hline 70 & APN & $\mathrm{CA}$ & 70 & APN & $\mathrm{C}$ & 2.475 \\
\hline 71 & ASP & $\mathrm{CA}$ & 71 & ASP & $\mathrm{C}$ & 0.367 \\
\hline 72 & LEU & $\mathrm{CA}$ & 72 & LEU & $\mathrm{C}$ & 0.732 \\
\hline 73 & LEU & $\mathrm{CA}$ & 73 & LEU & $\mathrm{C}$ & -6.924 \\
\hline 74 & PHE & $\mathrm{CA}$ & 74 & PHE & $\mathrm{C}$ & 4.530 \\
\hline 77 & VAL & $\mathrm{CA}$ & 77 & VAL & $\mathrm{C}$ & 2.571 \\
\hline 79 & ASP & $\mathrm{CA}$ & 79 & ASP & $\mathrm{C}$ & -5.637 \\
\hline 81 & GLN & $\mathrm{CA}$ & 81 & GLN & $\mathrm{C}$ & -2.988 \\
\hline 82 & SER & $\mathrm{CA}$ & 82 & SER & $\mathrm{C}$ & -0.767 \\
\hline 83 & LEU & $\mathrm{CA}$ & 83 & LEU & $\mathrm{C}$ & 1.164 \\
\hline 85 & TYR & $\mathrm{CA}$ & 85 & TYR & $\mathrm{C}$ & 0.023 \\
\hline 86 & SER & $\mathrm{CA}$ & 86 & SER & $\mathrm{C}$ & -3.298 \\
\hline 90 & ALA & $\mathrm{CA}$ & 90 & ALA & $\mathrm{C}$ & 7.086 \\
\hline 91 & GLN & $\mathrm{CA}$ & 91 & GLN & $\mathrm{C}$ & -1.244 \\
\hline 92 & ARG & $\mathrm{CA}$ & 92 & ARG & $\mathrm{C}$ & 0.217 \\
\hline 94 & GLY & $\mathrm{CA}$ & 94 & GLY & $\mathrm{C}$ & 2.992 \\
\hline 101 & ASP & $\mathrm{CA}$ & 101 & ASP & $\mathrm{C}$ & -2.307 \\
\hline 102 & ILE & $\mathrm{CA}$ & 102 & ILE & $\mathrm{C}$ & 51.908 \\
\hline 103 & LEU & $\mathrm{CA}$ & 103 & LEU & $\mathrm{C}$ & 2.348 \\
\hline 104 & LYS & $\mathrm{CA}$ & 104 & LYS & $\mathrm{C}$ & -1.997 \\
\hline 105 & ALA & $\mathrm{CA}$ & 105 & ALA & $\mathrm{C}$ & -3.310 \\
\hline 106 & LEU & $\mathrm{CA}$ & 106 & LEU & $\mathrm{C}$ & -0.756 \\
\hline 107 & APN & $\mathrm{CA}$ & 107 & APN & $\mathrm{C}$ & 3.349 \\
\hline 108 & GLY & $\mathrm{CA}$ & 108 & GLY & $\mathrm{C}$ & -1.693 \\
\hline 109 & GLU & $\mathrm{CA}$ & 109 & GLU & $\mathrm{C}$ & -2.021 \\
\hline 110 & GLU & $\mathrm{CA}$ & 110 & GLU & $\mathrm{C}$ & 4.691 \\
\hline 111 & APN & $\mathrm{CA}$ & 111 & $\mathrm{APN}$ & $\mathrm{C}$ & -0.212 \\
\hline 113 & ALA & $\mathrm{CA}$ & 113 & ALA & $\mathrm{C}$ & -4.221 \\
\hline 114 & ILE & $\mathrm{CA}$ & 114 & ILE & $\mathrm{C}$ & -0.802 \\
\hline
\end{tabular}


Table B.3 - continued from previous page

\begin{tabular}{|c|c|c|c|c|c|c|}
\hline Res: Number1 & Res: name1 & Atom1 & Res: Number2 & Res: name2 & Atom2 & D \\
\hline 120 & $\mathrm{ALA}$ & $\mathrm{CA}$ & 120 & ALA & $\mathrm{C}$ & 1.901 \\
\hline 122 & ALA & $\mathrm{CA}$ & 122 & ALA & $\mathrm{C}$ & -0.460 \\
\hline 124 & ARG & $\mathrm{CA}$ & 124 & ARG & $\mathrm{C}$ & -3.375 \\
\hline 125 & VAL & $\mathrm{CA}$ & 125 & VAL & $\mathrm{C}$ & -1.010 \\
\hline 126 & PHE & $\mathrm{CA}$ & 126 & PHE & $\mathrm{C}$ & -1.275 \\
\hline 130 & TYR & $\mathrm{CA}$ & 130 & TYR & $\mathrm{C}$ & 4.437 \\
\hline 131 & ASP & $\mathrm{CA}$ & 131 & ASP & $\mathrm{C}$ & 4.063 \\
\hline 132 & GLU & $\mathrm{CA}$ & 132 & GLU & $\mathrm{C}$ & -0.263 \\
\hline 133 & $\mathrm{APN}$ & $\mathrm{CA}$ & 133 & $\mathrm{APN}$ & $\mathrm{C}$ & -1.012 \\
\hline 135 & LYS & $\mathrm{CA}$ & 135 & LYS & $\mathrm{C}$ & 1.766 \\
\hline 137 & ILE & $\mathrm{CA}$ & 137 & ILE & $\mathrm{C}$ & -1.675 \\
\hline 138 & GLY & $\mathrm{CA}$ & 138 & GLY & $\mathrm{C}$ & -4.745 \\
\hline 139 & VAL & $\mathrm{CA}$ & 139 & VAL & $\mathrm{C}$ & 0.584 \\
\hline 140 & VAL & $\mathrm{CA}$ & 140 & VAL & $\mathrm{C}$ & -5.503 \\
\hline 141 & ALA & $\mathrm{CA}$ & 141 & $\mathrm{ALA}$ & $\mathrm{C}$ & -7.929 \\
\hline 142 & ILE & $\mathrm{CA}$ & 142 & ILE & $\mathrm{C}$ & -1.596 \\
\hline 143 & GLY & $\mathrm{CA}$ & 143 & GLY & $\mathrm{C}$ & 2.066 \\
\hline 144 & LEU & $\mathrm{CA}$ & 144 & LEU & $\mathrm{C}$ & 0.871 \\
\hline 148 & ARG & $\mathrm{CA}$ & 148 & $\mathrm{ARG}$ & $\mathrm{C}$ & -0.034 \\
\hline 150 & THR & $\mathrm{CA}$ & 150 & THR & $\mathrm{C}$ & 1.342 \\
\hline 151 & GLN & $\mathrm{CA}$ & 151 & GLN & $\mathrm{C}$ & 0.621 \\
\hline 152 & GLN & $\mathrm{CA}$ & 152 & GLN & $\mathrm{C}$ & -0.630 \\
\hline 153 & ILE & $\mathrm{CA}$ & 153 & ILE & $\mathrm{C}$ & -1.318 \\
\hline 154 & $\mathrm{APN}$ & $\mathrm{CA}$ & 154 & $\mathrm{APN}$ & $\mathrm{C}$ & -0.776 \\
\hline 155 & ASP & $\mathrm{CA}$ & 155 & ASP & $\mathrm{C}$ & -0.395 \\
\hline 156 & SER & $\mathrm{CA}$ & 156 & SER & $\mathrm{C}$ & -0.362 \\
\hline 23 & $\mathrm{ASP}$ & $\mathrm{CA}$ & 23 & ASP & $\mathrm{HA}$ & -1.992 \\
\hline 24 & MET & $\mathrm{CA}$ & 24 & MET & $\mathrm{HA}$ & -16.375 \\
\hline 25 & THR & $\mathrm{CA}$ & 25 & THR & $\mathrm{HA}$ & -25.682 \\
\hline 26 & ARG & $\mathrm{CA}$ & 26 & ARG & $\mathrm{HA}$ & -9.467 \\
\hline 27 & ASP & $\mathrm{CA}$ & 27 & ASP & $\mathrm{HA}$ & -14.372 \\
\hline 28 & GLY & $\mathrm{CA}$ & 28 & GLY & $\mathrm{HA}$ & -6.572 \\
\hline 31 & $\mathrm{APN}$ & $\mathrm{CA}$ & 31 & $\mathrm{APN}$ & $\mathrm{HA}$ & -21.858 \\
\hline 34 & LEU & $\mathrm{CA}$ & 34 & LEU & $\mathrm{HA}$ & -10.503 \\
\hline 35 & ALA & $\mathrm{CA}$ & 35 & ALA & $\mathrm{HA}$ & -28.208 \\
\hline 36 & VAL & $\mathrm{CA}$ & 36 & VAL & $\mathrm{HA}$ & -1.588 \\
\hline 37 & ALA & $\mathrm{CA}$ & 37 & $\mathrm{ALA}$ & $\mathrm{HA}$ & -6.806 \\
\hline 38 & ARG & $\mathrm{CA}$ & 38 & ARG & $\mathrm{HA}$ & -13.595 \\
\hline 40 & LEU & $\mathrm{CA}$ & 40 & LEU & $\mathrm{HA}$ & 10.515 \\
\hline 41 & ALA & $\mathrm{CA}$ & 41 & ALA & $\mathrm{HA}$ & -4.692 \\
\hline 42 & ASP & $\mathrm{CA}$ & 42 & ASP & HA & -23.383 \\
\hline 45 & GLU & $\mathrm{CA}$ & 45 & GLU & $\mathrm{HA}$ & -22.265 \\
\hline
\end{tabular}


Table B.3 - continued from previous page

\begin{tabular}{|c|c|c|c|c|c|c|}
\hline Res: Number1 & Res: name1 & Atom1 & Res: Number2 & Res: name2 & Atom2 & D \\
\hline 47 & ARG & $\mathrm{CA}$ & 47 & ARG & HA & 23.448 \\
\hline 48 & GLN & $\mathrm{CA}$ & 48 & GLN & HA & -35.860 \\
\hline 50 & LEU & $\mathrm{CA}$ & 50 & LEU & HA & 20.066 \\
\hline 51 & GLN & $\mathrm{CA}$ & 51 & GLN & HA & 9.372 \\
\hline 52 & LYS & $\mathrm{CA}$ & 52 & LYS & HA & -39.761 \\
\hline 58 & GLY & $\mathrm{CA}$ & 58 & GLY & HA & 33.644 \\
\hline 59 & ILE & $\mathrm{CA}$ & 59 & ILE & HA & -22.077 \\
\hline 60 & GLN & $\mathrm{CA}$ & 60 & GLN & HA & -7.230 \\
\hline 61 & ALA & $\mathrm{CA}$ & 61 & ALA & HA & -14.769 \\
\hline 62 & ILE & $\mathrm{CA}$ & 62 & ILE & HA & 22.705 \\
\hline 63 & ALA & $\mathrm{CA}$ & 63 & ALA & HA & 4.796 \\
\hline 64 & GLU & $\mathrm{CA}$ & 64 & GLU & HA & -11.438 \\
\hline 65 & ALA & $\mathrm{CA}$ & 65 & ALA & HA & -9.486 \\
\hline 66 & VAL & $\mathrm{CA}$ & 66 & VAL & HA & 28.202 \\
\hline 67 & ARG & $\mathrm{CA}$ & 67 & ARG & HA & 0.779 \\
\hline 68 & LYS & $\mathrm{CA}$ & 68 & LYS & HA & -1.144 \\
\hline 69 & ARG & $\mathrm{CA}$ & 69 & ARG & $\mathrm{HA}$ & -15.202 \\
\hline 70 & APN & $\mathrm{CA}$ & 70 & $\mathrm{APN}$ & HA & 7.626 \\
\hline 71 & ASP & $\mathrm{CA}$ & 71 & ASP & HA & -2.654 \\
\hline 72 & LEU & $\mathrm{CA}$ & 72 & LEU & HA & -15.433 \\
\hline 73 & LEU & $\mathrm{CA}$ & 73 & LEU & HA & -11.133 \\
\hline 74 & PHE & $\mathrm{CA}$ & 74 & PHE & HA & -35.971 \\
\hline 77 & VAL & $\mathrm{CA}$ & 77 & VAL & HA & -18.338 \\
\hline 79 & ASP & $\mathrm{CA}$ & 79 & ASP & HA & 33.309 \\
\hline 81 & GLN & $\mathrm{CA}$ & 81 & GLN & HA & 11.727 \\
\hline 82 & SER & $\mathrm{CA}$ & 82 & SER & HA & 14.098 \\
\hline 83 & LEU & $\mathrm{CA}$ & 83 & LEU & HA & 20.346 \\
\hline 85 & TYR & $\mathrm{CA}$ & 85 & TYR & HA & -11.966 \\
\hline 86 & SER & $\mathrm{CA}$ & 86 & SER & HA & -1.462 \\
\hline 90 & ALA & $\mathrm{CA}$ & 90 & ALA & HA & 13.911 \\
\hline 91 & GLN & $\mathrm{CA}$ & 91 & GLN & $\mathrm{HA}$ & -27.534 \\
\hline 92 & ARG & $\mathrm{CA}$ & 92 & ARG & HA & 31.162 \\
\hline 94 & GLY & $\mathrm{CA}$ & 94 & GLY & HA & -11.802 \\
\hline 103 & LEU & $\mathrm{CA}$ & 103 & LEU & HA & -39.398 \\
\hline 104 & LYS & $\mathrm{CA}$ & 104 & LYS & HA & -7.715 \\
\hline 105 & ALA & $\mathrm{CA}$ & 105 & ALA & HA & 15.062 \\
\hline 106 & LEU & $\mathrm{CA}$ & 106 & LEU & HA & 4.954 \\
\hline 108 & GLY & $\mathrm{CA}$ & 108 & GLY & HA & -4.875 \\
\hline 109 & GLU & $\mathrm{CA}$ & 109 & GLU & HA & -3.847 \\
\hline 110 & GLU & $\mathrm{CA}$ & 110 & GLU & HA & 3.242 \\
\hline 111 & APN & $\mathrm{CA}$ & 111 & APN & HA & -8.163 \\
\hline 113 & ALA & $\mathrm{CA}$ & 113 & ALA & HA & -10.839 \\
\hline
\end{tabular}


Table B.3 - continued from previous page

\begin{tabular}{|c|c|c|c|c|c|c|}
\hline Res: Number1 & Res: name1 & Atom1 & Res: Number2 & Res: name2 & Atom2 & D \\
\hline 114 & ILE & $\mathrm{CA}$ & 114 & ILE & $\mathrm{HA}$ & 24.258 \\
\hline 120 & ALA & $\mathrm{CA}$ & 120 & ALA & HA & 30.245 \\
\hline 122 & ALA & $\mathrm{CA}$ & 122 & ALA & HA & -15.533 \\
\hline 124 & $\mathrm{ARG}$ & $\mathrm{CA}$ & 124 & $\mathrm{ARG}$ & $\mathrm{HA}$ & 23.059 \\
\hline 125 & VAL & $\mathrm{CA}$ & 125 & VAL & $\mathrm{HA}$ & 12.949 \\
\hline 130 & TYR & $\mathrm{CA}$ & 130 & TYR & HA & 30.152 \\
\hline 131 & ASP & $\mathrm{CA}$ & 131 & ASP & HA & -3.947 \\
\hline 132 & GLU & $\mathrm{CA}$ & 132 & GLU & $\mathrm{HA}$ & -28.927 \\
\hline 133 & $\mathrm{APN}$ & $\mathrm{CA}$ & 133 & $\mathrm{APN}$ & HA & 35.768 \\
\hline 135 & LYS & $\mathrm{CA}$ & 135 & LYS & $\mathrm{HA}$ & 1.344 \\
\hline 137 & ILE & $\mathrm{CA}$ & 137 & ILE & $\mathrm{HA}$ & 8.414 \\
\hline 138 & GLY & $\mathrm{CA}$ & 138 & GLY & $\mathrm{HA}$ & 25.908 \\
\hline 140 & VAL & $\mathrm{CA}$ & 140 & VAL & HA & 5.031 \\
\hline 141 & ALA & $\mathrm{CA}$ & 141 & ALA & $\mathrm{HA}$ & -15.067 \\
\hline 142 & ILE & $\mathrm{CA}$ & 142 & ILE & $\mathrm{HA}$ & -7.068 \\
\hline 143 & GLY & $\mathrm{CA}$ & 143 & GLY & $\mathrm{HA}$ & 20.430 \\
\hline 148 & ARG & $\mathrm{CA}$ & 148 & ARG & $\mathrm{HA}$ & -23.074 \\
\hline 150 & THR & $\mathrm{CA}$ & 150 & THR & $\mathrm{HA}$ & -0.293 \\
\hline 151 & GLN & $\mathrm{CA}$ & 151 & GLN & $\mathrm{HA}$ & -5.697 \\
\hline 152 & GLN & $\mathrm{CA}$ & 152 & GLN & $\mathrm{HA}$ & -4.922 \\
\hline 153 & ILE & $\mathrm{CA}$ & 153 & ILE & $\mathrm{HA}$ & 9.942 \\
\hline 154 & $\mathrm{APN}$ & $\mathrm{CA}$ & 154 & $\mathrm{APN}$ & $\mathrm{HA}$ & -0.997 \\
\hline 155 & $\mathrm{ASP}$ & $\mathrm{CA}$ & 155 & $\mathrm{ASP}$ & $\mathrm{HA}$ & -0.381 \\
\hline 156 & SER & $\mathrm{CA}$ & 156 & SER & $\mathrm{HA}$ & -3.383 \\
\hline
\end{tabular}

Table B.4: Residual dipolar couplings measured for Ubiquitine in Otting phase

\begin{tabular}{|c|c|c|c|c|c|c|}
\hline Res: Number 1 & Res: name1 & Atom1 & Res: Number2 & Res: name2 & Atom2 & $\mathbf{D}$ \\
\hline 3 & ILE & $\mathrm{HN}$ & 3 & ILE & $\mathrm{N}$ & 2.987 \\
\hline 4 & PHE & $\mathrm{HN}$ & 4 & PHE & $\mathrm{N}$ & 2.739 \\
\hline 5 & VAL & $\mathrm{HN}$ & 5 & VAL & $\mathrm{N}$ & 1.794 \\
\hline 6 & LYS & $\mathrm{HN}$ & 6 & LYS & $\mathrm{N}$ & 2.311 \\
\hline 7 & THR & $\mathrm{HN}$ & 7 & THR & $\mathrm{N}$ & -2.102 \\
\hline 8 & LEU & $\mathrm{HN}$ & 8 & LEU & $\mathrm{N}$ & 1.744 \\
\hline 10 & GLY & $\mathrm{HN}$ & 10 & GLY & $\mathrm{N}$ & 1.635 \\
\hline 11 & LYS & $\mathrm{HN}$ & 11 & LYS & $\mathrm{N}$ & -6.481 \\
\hline 12 & THR & $\mathrm{HN}$ & 12 & THR & $\mathrm{N}$ & 0.310 \\
\hline 13 & ILE & $\mathrm{HN}$ & 13 & ILE & $\mathrm{N}$ & 0.076 \\
\hline 14 & THR & $\mathrm{HN}$ & 14 & THR & $\mathrm{N}$ & 2.885 \\
\hline 15 & $\mathrm{LEU}$ & $\mathrm{HN}$ & 15 & LEU & $\mathrm{N}$ & 3.678 \\
\hline
\end{tabular}


Table B.4 - continued from previous page

\begin{tabular}{|c|c|c|c|c|c|c|}
\hline Res: Number1 & Res: name1 & Atom1 & Res: Number2 & Res: name2 & Atom2 & $\mathbf{D}$ \\
\hline 16 & GLU & $\mathrm{HN}$ & 16 & GLU & $\mathrm{N}$ & -0.570 \\
\hline 17 & VAL & $\mathrm{HN}$ & 17 & VAL & $\mathrm{N}$ & -1.804 \\
\hline 18 & GLU & $\mathrm{HN}$ & 18 & GLU & $\mathrm{N}$ & -7.763 \\
\hline 20 & SER & $\mathrm{HN}$ & 20 & SER & $\mathrm{N}$ & -3.474 \\
\hline 21 & ASP & $\mathrm{HN}$ & 21 & ASP & $\mathrm{N}$ & -1.734 \\
\hline 22 & THR & $\mathrm{HN}$ & 22 & THR & $\mathrm{N}$ & 5.953 \\
\hline 23 & ILE & $\mathrm{HN}$ & 23 & ILE & $\mathrm{N}$ & 2.506 \\
\hline 25 & APN & $\mathrm{HN}$ & 25 & $\mathrm{APN}$ & $\mathrm{N}$ & 1.278 \\
\hline 26 & VAL & $\mathrm{HN}$ & 26 & VAL & $\mathrm{N}$ & 2.015 \\
\hline 27 & LYS & $\mathrm{HN}$ & 27 & LYS & $\mathrm{N}$ & 2.420 \\
\hline 28 & ALA & $\mathrm{HN}$ & 28 & ALA & $\mathrm{N}$ & 2.000 \\
\hline 29 & LYS & $\mathrm{HN}$ & 29 & LYS & $\mathrm{N}$ & 2.551 \\
\hline 30 & THR & $\mathrm{HN}$ & 30 & THR & $\mathrm{N}$ & 1.777 \\
\hline 31 & GLN & HN & 31 & GLN & $\mathrm{N}$ & 2.870 \\
\hline 32 & ASP & $\mathrm{HN}$ & 32 & ASP & $\mathrm{N}$ & 1.493 \\
\hline 33 & LYS & $\mathrm{HN}$ & 33 & LYS & $\mathrm{N}$ & 2.092 \\
\hline 34 & GLU & $\mathrm{HN}$ & 34 & GLU & $\mathrm{N}$ & 2.579 \\
\hline 35 & GLY & HN & 35 & GLY & $\mathrm{N}$ & -1.577 \\
\hline 36 & ILE & HN & 36 & ILE & $\mathrm{N}$ & -7.862 \\
\hline 39 & ASP & HN & 39 & ASP & $\mathrm{N}$ & 4.810 \\
\hline 40 & GLN & $\mathrm{HN}$ & 40 & GLN & $\mathrm{N}$ & -0.011 \\
\hline 41 & GLN & $\mathrm{HN}$ & 41 & GLN & $\mathrm{N}$ & 6.743 \\
\hline 42 & ARG & HN & 42 & ARG & $\mathrm{N}$ & 3.941 \\
\hline 43 & LEU & $\mathrm{HN}$ & 43 & LEU & $\mathrm{N}$ & 3.421 \\
\hline 44 & ILE & HN & 44 & ILE & $\mathrm{N}$ & 2.577 \\
\hline 45 & PHE & $\mathrm{HN}$ & 45 & PHE & $\mathrm{N}$ & -1.870 \\
\hline 46 & ALA & HN & 46 & ALA & $\mathrm{N}$ & 0.263 \\
\hline 47 & GLY & HN & 47 & GLY & $\mathrm{N}$ & 2.258 \\
\hline 49 & GLN & HN & 49 & GLN & $\mathrm{N}$ & -1.110 \\
\hline 50 & LEU & $\mathrm{HN}$ & 50 & LEU & $\mathrm{N}$ & 2.277 \\
\hline 51 & GLU & HN & 51 & GLU & $\mathrm{N}$ & -3.101 \\
\hline 52 & ASP & HN & 52 & ASP & $\mathrm{N}$ & -6.940 \\
\hline 54 & ARG & HN & 54 & ARG & $\mathrm{N}$ & 0.358 \\
\hline 55 & THR & HN & 55 & THR & $\mathrm{N}$ & 2.420 \\
\hline 56 & LEU & HN & 56 & LEU & $\mathrm{N}$ & 5.443 \\
\hline 57 & SER & HN & 57 & SER & $\mathrm{N}$ & 6.892 \\
\hline 58 & ASP & HN & 58 & ASP & $\mathrm{N}$ & 3.056 \\
\hline 59 & TYR & HN & 59 & TYR & $\mathrm{N}$ & 3.019 \\
\hline 60 & $\mathrm{APN}$ & $\mathrm{HN}$ & 60 & $\mathrm{APN}$ & $\mathrm{N}$ & 6.967 \\
\hline 61 & ILE & $\mathrm{HN}$ & 61 & ILE & $\mathrm{N}$ & 3.106 \\
\hline 62 & GLN & HN & 62 & GLN & $\mathrm{N}$ & 4.501 \\
\hline 63 & LYS & HN & 63 & LYS & $\mathrm{N}$ & -1.799 \\
\hline
\end{tabular}


Table B.4 - continued from previous page

\begin{tabular}{|c|c|c|c|c|c|c|}
\hline Res: Number1 & Res: name1 & Atom1 & Res: Number2 & Res: name2 & Atom2 & D \\
\hline 64 & GLU & $\mathrm{HN}$ & 64 & GLU & $\mathrm{N}$ & 5.003 \\
\hline 65 & SER & $\mathrm{HN}$ & 65 & SER & $\mathrm{N}$ & 6.272 \\
\hline 67 & LEU & $\mathrm{HN}$ & 67 & LEU & $\mathrm{N}$ & 2.036 \\
\hline 68 & HIS & $\mathrm{HN}$ & 68 & HIS & $\mathrm{N}$ & 1.821 \\
\hline 69 & LEU & $\mathrm{HN}$ & 69 & LEU & $\mathrm{N}$ & 0.880 \\
\hline 70 & VAL & $\mathrm{HN}$ & 70 & VAL & $\mathrm{N}$ & 5.007 \\
\hline 71 & LEU & $\mathrm{HN}$ & 71 & LEU & $\mathrm{N}$ & 5.932 \\
\hline 72 & ARG & $\mathrm{HN}$ & 72 & ARG & $\mathrm{N}$ & 3.477 \\
\hline 74 & ARG & $\mathrm{HN}$ & 74 & ARG & $\mathrm{N}$ & 2.760 \\
\hline 2 & GLN & $\mathrm{C}$ & 3 & ILE & $\mathrm{N}$ & -1.053 \\
\hline 3 & ILE & $\mathrm{C}$ & 4 & PHE & $\mathrm{N}$ & 0.497 \\
\hline 4 & PHE & $\mathrm{C}$ & 5 & VAL & $\mathrm{N}$ & -0.330 \\
\hline 5 & VAL & $\mathrm{C}$ & 6 & LYS & $\mathrm{N}$ & 0.344 \\
\hline 6 & LYS & $\mathrm{C}$ & 7 & THR & $\mathrm{N}$ & 0.202 \\
\hline 7 & THR & $\mathrm{C}$ & 8 & LEU & $\mathrm{N}$ & -0.844 \\
\hline 9 & THR & $\mathrm{C}$ & 10 & GLY & $\mathrm{N}$ & -0.222 \\
\hline 10 & GLY & $\mathrm{C}$ & 11 & LYS & $\mathrm{N}$ & 0.509 \\
\hline 11 & LYS & $\mathrm{C}$ & 12 & THR & $\mathrm{N}$ & -0.851 \\
\hline 12 & THR & $\mathrm{C}$ & 13 & ILE & $\mathrm{N}$ & 0.432 \\
\hline 13 & ILE & $\mathrm{C}$ & 14 & THR & $\mathrm{N}$ & -0.254 \\
\hline 14 & THR & $\mathrm{C}$ & 15 & LEU & $\mathrm{N}$ & 0.208 \\
\hline 15 & LEU & $\mathrm{C}$ & 16 & GLU & $\mathrm{N}$ & 0.788 \\
\hline 16 & GLU & $\mathrm{C}$ & 17 & VAL & $\mathrm{N}$ & 0.182 \\
\hline 17 & VAL & $\mathrm{C}$ & 18 & GLU & $\mathrm{N}$ & 0.345 \\
\hline 19 & PRO & $\mathrm{C}$ & 20 & SER & $\mathrm{N}$ & 0.785 \\
\hline 20 & SER & $\mathrm{C}$ & 21 & ASP & $\mathrm{N}$ & 0.416 \\
\hline 21 & ASP & $\mathrm{C}$ & 22 & THR & $\mathrm{N}$ & -0.645 \\
\hline 22 & THR & $\mathrm{C}$ & 23 & ILE & $\mathrm{N}$ & -0.062 \\
\hline 24 & GLU & $\mathrm{C}$ & 25 & ASN & $\mathrm{N}$ & -0.805 \\
\hline 25 & ASN & $\mathrm{C}$ & 26 & VAL & $\mathrm{N}$ & 0.691 \\
\hline 26 & VAL & $\mathrm{C}$ & 27 & LYS & $\mathrm{N}$ & -0.968 \\
\hline 27 & LYS & $\mathrm{C}$ & 28 & ALA & $\mathrm{N}$ & 0.683 \\
\hline 28 & ALA & $\mathrm{C}$ & 29 & LYS & $\mathrm{N}$ & -0.674 \\
\hline 29 & LYS & $\mathrm{C}$ & 30 & THR & $\mathrm{N}$ & 0.115 \\
\hline 30 & THR & $\mathrm{C}$ & 31 & GLN & $\mathrm{N}$ & 0.069 \\
\hline 31 & GLN & $\mathrm{C}$ & 32 & ASP & $\mathrm{N}$ & -0.533 \\
\hline 32 & ASP & $\mathrm{C}$ & 33 & LYS & $\mathrm{N}$ & 0.510 \\
\hline 33 & LYS & $\mathrm{C}$ & 34 & PHE & $\mathrm{N}$ & -0.457 \\
\hline 34 & PHE & $\mathrm{C}$ & 35 & GLY & $\mathrm{N}$ & 0.672 \\
\hline 35 & GLY & $\mathrm{C}$ & 36 & ILE & $\mathrm{N}$ & 0.381 \\
\hline 38 & PRO & $\mathrm{C}$ & 39 & ASP & $\mathrm{N}$ & -0.933 \\
\hline 39 & ASP & $\mathrm{C}$ & 40 & GLN & $\mathrm{N}$ & 0.175 \\
\hline
\end{tabular}


Table B.4 - continued from previous page

\begin{tabular}{|c|c|c|c|c|c|c|}
\hline Res: Number1 & Res: name1 & Atom1 & Res: Number2 & Res: name2 & Atom2 & $\mathbf{D}$ \\
\hline 40 & GLN & $\mathrm{C}$ & 41 & GLN & $\mathrm{N}$ & -0.568 \\
\hline 41 & GLN & $\mathrm{C}$ & 42 & ARG & $\mathrm{N}$ & 0.717 \\
\hline 42 & ARG & $\mathrm{C}$ & 43 & LEU & $\mathrm{N}$ & -0.126 \\
\hline 43 & LEU & $\mathrm{C}$ & 44 & ILE & $\mathrm{N}$ & 0.303 \\
\hline 44 & ILE & $\mathrm{C}$ & 45 & PHE & $\mathrm{N}$ & 0.135 \\
\hline 45 & PHE & $\mathrm{C}$ & 46 & ALA & $\mathrm{N}$ & 0.392 \\
\hline 46 & ALA & $\mathrm{C}$ & 47 & GLY & $\mathrm{N}$ & -0.615 \\
\hline 47 & GLY & $\mathrm{C}$ & 48 & LYS & $\mathrm{N}$ & 0.631 \\
\hline 48 & LYS & $\mathrm{C}$ & 49 & GLN & $\mathrm{N}$ & -0.760 \\
\hline 49 & GLN & $\mathrm{C}$ & 50 & LEU & $\mathrm{N}$ & 0.235 \\
\hline 50 & LEU & $\mathrm{C}$ & 51 & GLU & $\mathrm{N}$ & 0.466 \\
\hline 51 & GLU & $\mathrm{C}$ & 52 & ASP & $\mathrm{N}$ & -0.184 \\
\hline 53 & GLY & $\mathrm{C}$ & 54 & ARG & $\mathrm{N}$ & 0.575 \\
\hline 54 & ARG & $\mathrm{C}$ & 55 & THR & $\mathrm{N}$ & -0.969 \\
\hline 55 & THR & $\mathrm{C}$ & 56 & LEU & $\mathrm{N}$ & -0.250 \\
\hline 56 & LEU & $\mathrm{C}$ & 57 & SER & $\mathrm{N}$ & 0.214 \\
\hline 57 & SER & $\mathrm{C}$ & 58 & ASP & $\mathrm{N}$ & -0.724 \\
\hline 58 & ASP & $\mathrm{C}$ & 59 & TYR & $\mathrm{N}$ & 0.271 \\
\hline 59 & TYR & $\mathrm{C}$ & 60 & APN & $\mathrm{N}$ & -0.539 \\
\hline 60 & APN & $\mathrm{C}$ & 61 & ILE & $\mathrm{N}$ & 0.239 \\
\hline 61 & ILE & $\mathrm{C}$ & 62 & GLN & $\mathrm{N}$ & -0.045 \\
\hline 62 & GLN & $\mathrm{C}$ & 63 & LYS & $\mathrm{N}$ & -0.798 \\
\hline 63 & LYS & $\mathrm{C}$ & 64 & GLU & $\mathrm{N}$ & -0.016 \\
\hline 64 & GLU & $\mathrm{C}$ & 65 & SER & $\mathrm{N}$ & -0.235 \\
\hline 66 & THR & $\mathrm{C}$ & 67 & LEU & $\mathrm{N}$ & 0.052 \\
\hline 67 & LEU & $\mathrm{C}$ & 68 & HIS & $\mathrm{N}$ & -0.858 \\
\hline 68 & HIS & $\mathrm{C}$ & 69 & LEU & $\mathrm{N}$ & 0.073 \\
\hline 69 & LEU & $\mathrm{C}$ & 70 & VAL & $\mathrm{N}$ & -0.598 \\
\hline 70 & VAL & $\mathrm{C}$ & 71 & LEU & $\mathrm{N}$ & -0.428 \\
\hline 71 & LEU & $\mathrm{C}$ & 72 & ARG & $\mathrm{N}$ & 0.343 \\
\hline 72 & ARG & $\mathrm{C}$ & 73 & LEU & $\mathrm{N}$ & -0.069 \\
\hline 75 & GLY & $\mathrm{C}$ & 76 & GLY & $\mathrm{N}$ & -0.308 \\
\hline 1 & MET & $\mathrm{CA}$ & 1 & MET & $\mathrm{C}$ & 0.300 \\
\hline 2 & GLN & $\mathrm{CA}$ & 2 & GLN & $\mathrm{C}$ & 0.266 \\
\hline 3 & ILE & $\mathrm{CA}$ & 3 & ILE & $\mathrm{C}$ & 0.538 \\
\hline 4 & PHE & $\mathrm{CA}$ & 4 & PHE & $\mathrm{C}$ & -2.016 \\
\hline 5 & VAL & $\mathrm{CA}$ & 5 & VAL & $\mathrm{C}$ & 1.135 \\
\hline 6 & LYS & $\mathrm{CA}$ & 6 & LYS & $\mathrm{C}$ & -2.363 \\
\hline 7 & THR & $\mathrm{CA}$ & 7 & THR & $\mathrm{C}$ & 1.965 \\
\hline 8 & LEU & $\mathrm{CA}$ & 8 & LEU & $\mathrm{C}$ & 0.728 \\
\hline 9 & THR & $\mathrm{CA}$ & 9 & THR & $\mathrm{C}$ & -2.232 \\
\hline 10 & GLY & $\mathrm{CA}$ & 10 & GLY & $\mathrm{C}$ & 0.749 \\
\hline
\end{tabular}


Table B.4 - continued from previous page

\begin{tabular}{|c|c|c|c|c|c|c|}
\hline Res: Number1 & Res: name1 & Atom1 & Res: Number2 & Res: name2 & Atom2 & $\mathbf{D}$ \\
\hline 11 & LYS & $\mathrm{CA}$ & 11 & LYS & $\mathrm{C}$ & 1.065 \\
\hline 12 & THR & $\mathrm{CA}$ & 12 & THR & $\mathrm{C}$ & 1.844 \\
\hline 13 & ILE & $\mathrm{CA}$ & 13 & ILE & $\mathrm{C}$ & -0.195 \\
\hline 14 & THR & $\mathrm{CA}$ & 14 & THR & $\mathrm{C}$ & 1.641 \\
\hline 15 & LEU & $\mathrm{CA}$ & 15 & LEU & $\mathrm{C}$ & -0.997 \\
\hline 16 & GLU & $\mathrm{CA}$ & 16 & GLU & $\mathrm{C}$ & 0.552 \\
\hline 17 & VAL & $\mathrm{CA}$ & 17 & VAL & $\mathrm{C}$ & -0.054 \\
\hline 19 & PRO & $\mathrm{CA}$ & 19 & PRO & $\mathrm{C}$ & -0.253 \\
\hline 20 & SER & $\mathrm{CA}$ & 20 & SER & $\mathrm{C}$ & -0.916 \\
\hline 21 & ASP & $\mathrm{CA}$ & 21 & ASP & $\mathrm{C}$ & -0.498 \\
\hline 22 & THR & $\mathrm{CA}$ & 22 & THR & $\mathrm{C}$ & 0.571 \\
\hline 24 & GLU & $\mathrm{CA}$ & 24 & GLU & $\mathrm{C}$ & -0.955 \\
\hline 25 & ASN & $\mathrm{CA}$ & 25 & $\mathrm{ASN}$ & $\mathrm{C}$ & 1.198 \\
\hline 26 & VAL & $\mathrm{CA}$ & 26 & VAL & $\mathrm{C}$ & -1.366 \\
\hline 27 & LYS & $\mathrm{CA}$ & 27 & LYS & $\mathrm{C}$ & 1.140 \\
\hline 28 & ALA & $\mathrm{CA}$ & 28 & ALA & $\mathrm{C}$ & -1.350 \\
\hline 29 & LYS & $\mathrm{CA}$ & 29 & LYS & $\mathrm{C}$ & 1.245 \\
\hline 30 & ILE & $\mathrm{CA}$ & 30 & ILE & $\mathrm{C}$ & -0.283 \\
\hline 31 & GLN & $\mathrm{CA}$ & 31 & GLN & $\mathrm{C}$ & 0.647 \\
\hline 32 & ASP & $\mathrm{CA}$ & 32 & ASP & $\mathrm{C}$ & 0.446 \\
\hline 34 & GLU & $\mathrm{CA}$ & 34 & GLU & $\mathrm{C}$ & -2.371 \\
\hline 35 & GLY & $\mathrm{CA}$ & 35 & GLY & $\mathrm{C}$ & -0.449 \\
\hline 38 & PRO & $\mathrm{CA}$ & 38 & PRO & $\mathrm{C}$ & -0.198 \\
\hline 39 & ASP & $\mathrm{CA}$ & 39 & ASP & $\mathrm{C}$ & 2.411 \\
\hline 40 & GLN & $\mathrm{CA}$ & 40 & GLN & $\mathrm{C}$ & -0.894 \\
\hline 41 & GLN & $\mathrm{CA}$ & 41 & GLN & $\mathrm{C}$ & 0.167 \\
\hline 42 & $\mathrm{ARG}$ & $\mathrm{CA}$ & 42 & $\mathrm{ARG}$ & $\mathrm{C}$ & -2.423 \\
\hline 43 & LEU & $\mathrm{CA}$ & 43 & LEU & $\mathrm{C}$ & 1.252 \\
\hline 44 & ILE & $\mathrm{CA}$ & 44 & ILE & $\mathrm{C}$ & -1.881 \\
\hline 45 & PHE & $\mathrm{CA}$ & 45 & PHE & $\mathrm{C}$ & 0.619 \\
\hline 46 & ALA & $\mathrm{CA}$ & 46 & ALA & $\mathrm{C}$ & -2.375 \\
\hline 47 & GLY & $\mathrm{CA}$ & 47 & GLY & $\mathrm{C}$ & -0.252 \\
\hline 49 & GLN & $\mathrm{CA}$ & 49 & GLN & $\mathrm{C}$ & 1.090 \\
\hline 50 & LEU & $\mathrm{CA}$ & 50 & LEU & $\mathrm{C}$ & -0.571 \\
\hline 51 & GLU & $\mathrm{CA}$ & 51 & GLU & $\mathrm{C}$ & 0.743 \\
\hline 53 & GLY & $\mathrm{CA}$ & 53 & GLY & $\mathrm{C}$ & -1.362 \\
\hline 54 & ARG & $\mathrm{CA}$ & 54 & ARG & $\mathrm{C}$ & -1.221 \\
\hline 55 & THR & $\mathrm{CA}$ & 55 & THR & $\mathrm{C}$ & 1.073 \\
\hline 56 & LEU & $\mathrm{CA}$ & 56 & LEU & $\mathrm{C}$ & -0.944 \\
\hline 58 & ASP & $\mathrm{CA}$ & 58 & ASP & $\mathrm{C}$ & 1.915 \\
\hline 60 & $\mathrm{ASN}$ & $\mathrm{CA}$ & 60 & $\mathrm{ASN}$ & $\mathrm{C}$ & 0.514 \\
\hline 61 & ILE & $\mathrm{CA}$ & 61 & ILE & $\mathrm{C}$ & -1.242 \\
\hline
\end{tabular}


Table B.4 - continued from previous page

\begin{tabular}{|c|c|c|c|c|c|c|}
\hline Res: Number1 & Res: name1 & Atom1 & Res: Number2 & Res: name2 & Atom2 & $\mathbf{D}$ \\
\hline 62 & GLN & $\mathrm{CA}$ & 62 & GLN & $\mathrm{C}$ & 1.043 \\
\hline 63 & LYS & $\mathrm{CA}$ & 63 & LYS & $\mathrm{C}$ & 1.568 \\
\hline 64 & GLU & $\mathrm{CA}$ & 64 & GLU & $\mathrm{C}$ & -0.037 \\
\hline 67 & LEU & $\mathrm{CA}$ & 67 & $\mathrm{LEU}$ & $\mathrm{C}$ & 0.592 \\
\hline 69 & LEU & $\mathrm{CA}$ & 69 & LEU & $\mathrm{C}$ & -0.414 \\
\hline 70 & VAL & $\mathrm{CA}$ & 70 & VAL & $\mathrm{C}$ & 0.242 \\
\hline 71 & LEU & $\mathrm{CA}$ & 71 & LEU & $\mathrm{C}$ & -1.991 \\
\hline 72 & ARG & $\mathrm{CA}$ & 72 & ARG & $\mathrm{C}$ & -0.276 \\
\hline 74 & ARG & $\mathrm{CA}$ & 74 & ARG & $\mathrm{C}$ & -1.069 \\
\hline 2 & GLN & $\mathrm{CA}$ & 2 & GLN & HA & -1.938 \\
\hline 3 & ILE & $\mathrm{CA}$ & 3 & ILE & HA & 0.761 \\
\hline 4 & PHE & $\mathrm{CA}$ & 4 & PHE & HA & 10.574 \\
\hline 5 & VAL & $\mathrm{CA}$ & 5 & VAL & HA & 10.108 \\
\hline 6 & LYS & $\mathrm{CA}$ & 6 & LYS & HA & -0.207 \\
\hline 7 & THR & $\mathrm{CA}$ & 7 & THR & HA & 2.327 \\
\hline 8 & LEU & $\mathrm{CA}$ & 8 & LEU & HA & 1.712 \\
\hline 9 & THR & $\mathrm{CA}$ & 9 & THR & HA & 7.423 \\
\hline 10 & GLY & $\mathrm{CA}$ & 10 & GLY & HA & 17.939 \\
\hline 11 & LYS & $\mathrm{CA}$ & 11 & LYS & HA & -5.744 \\
\hline 12 & THR & $\mathrm{CA}$ & 12 & THR & HA & -10.440 \\
\hline 13 & ILE & $\mathrm{CA}$ & 13 & ILE & HA & 2.252 \\
\hline 14 & THR & $\mathrm{CA}$ & 14 & THR & HA & 10.185 \\
\hline 15 & LEU & $\mathrm{CA}$ & 15 & LEU & HA & 9.290 \\
\hline 16 & GLU & $\mathrm{CA}$ & 16 & GLU & $\mathrm{HA}$ & 5.156 \\
\hline 17 & VAL & $\mathrm{CA}$ & 17 & VAL & HA & 8.181 \\
\hline 19 & PRO & $\mathrm{CA}$ & 19 & PRO & HA & 11.370 \\
\hline 20 & SER & $\mathrm{CA}$ & 20 & SER & HA & 1.758 \\
\hline 21 & ASP & $\mathrm{CA}$ & 21 & ASP & HA & 17.148 \\
\hline 25 & ASN & $\mathrm{CA}$ & 25 & ASN & HA & 8.169 \\
\hline 26 & VAL & $\mathrm{CA}$ & 26 & VAL & HA & -12.486 \\
\hline 27 & LYS & $\mathrm{CA}$ & 27 & LYS & HA & 13.326 \\
\hline 28 & ALA & $\mathrm{CA}$ & 28 & ALA & HA & -20.446 \\
\hline 29 & LYS & $\mathrm{CA}$ & 29 & LYS & HA & 10.793 \\
\hline 30 & ILE & $\mathrm{CA}$ & 30 & ILE & HA & -8.764 \\
\hline 31 & GLN & $\mathrm{CA}$ & 31 & GLN & HA & 5.350 \\
\hline 32 & ASP & $\mathrm{CA}$ & 32 & ASP & HA & -0.250 \\
\hline 34 & GLU & $\mathrm{CA}$ & 34 & GLU & HA & 3.817 \\
\hline 35 & GLY & $\mathrm{CA}$ & 35 & GLY & HA & 24.092 \\
\hline 38 & PRO & $\mathrm{CA}$ & 38 & PRO & HA & -7.371 \\
\hline 39 & ASP & $\mathrm{CA}$ & 39 & ASP & HA & 2.435 \\
\hline 40 & GLN & $\mathrm{CA}$ & 40 & GLN & HA & -9.823 \\
\hline 41 & GLN & $\mathrm{CA}$ & 41 & GLN & HA & 14.735 \\
\hline
\end{tabular}


Table B.4 - continued from previous page

\begin{tabular}{|c|c|c|c|c|c|c|}
\hline Res: Number1 & Res: name1 & Atom1 & Res: Number2 & Res: name2 & Atom2 & D \\
\hline 42 & ARG & $\mathrm{CA}$ & 42 & ARG & $\mathrm{HA}$ & 19.047 \\
\hline 43 & LEU & $\mathrm{CA}$ & 43 & LEU & $\mathrm{HA}$ & 8.906 \\
\hline 44 & ILE & $\mathrm{CA}$ & 44 & ILE & $\mathrm{HA}$ & 1.254 \\
\hline 45 & PHE & $\mathrm{CA}$ & 45 & PHE & $\mathrm{HA}$ & 4.671 \\
\hline 46 & ALA & $\mathrm{CA}$ & 46 & ALA & $\mathrm{HA}$ & 14.170 \\
\hline 47 & GLY & $\mathrm{CA}$ & 47 & GLY & $\mathrm{HA}$ & 12.662 \\
\hline 48 & LYS & $\mathrm{CA}$ & 48 & LYS & $\mathrm{HA}$ & -11.402 \\
\hline 49 & GLN & $\mathrm{CA}$ & 49 & GLN & $\mathrm{HA}$ & -0.511 \\
\hline 50 & LEU & $\mathrm{CA}$ & 50 & LEU & $\mathrm{HA}$ & -0.798 \\
\hline 51 & GLU & $\mathrm{CA}$ & 51 & GLU & $\mathrm{HA}$ & -12.923 \\
\hline 53 & GLY & $\mathrm{CA}$ & 53 & GLY & $\mathrm{HA}$ & 8.942 \\
\hline 54 & $\mathrm{ARG}$ & $\mathrm{CA}$ & 54 & ARG & $\mathrm{HA}$ & 7.065 \\
\hline 55 & THR & $\mathrm{CA}$ & 55 & THR & $\mathrm{HA}$ & 4.232 \\
\hline 56 & LEU & $\mathrm{CA}$ & 56 & LEU & $\mathrm{HA}$ & 1.702 \\
\hline 57 & SER & $\mathrm{CA}$ & 57 & SER & $\mathrm{HA}$ & -1.862 \\
\hline 58 & ASP & $\mathrm{CA}$ & 58 & ASP & $\mathrm{HA}$ & 1.291 \\
\hline 59 & TYR & $\mathrm{CA}$ & 59 & TYR & $\mathrm{HA}$ & 3.033 \\
\hline 60 & $\mathrm{ASN}$ & $\mathrm{CA}$ & 60 & $\mathrm{ASN}$ & $\mathrm{HA}$ & 2.074 \\
\hline 61 & ILE & $\mathrm{CA}$ & 61 & ILE & $\mathrm{HA}$ & 20.333 \\
\hline 62 & GLN & $\mathrm{CA}$ & 62 & GLN & $\mathrm{HA}$ & 15.636 \\
\hline 63 & LYS & $\mathrm{CA}$ & 63 & LYS & $\mathrm{HA}$ & 0.779 \\
\hline 64 & GLU & $\mathrm{CA}$ & 64 & GLU & $\mathrm{HA}$ & 3.271 \\
\hline 65 & SER & $\mathrm{CA}$ & 65 & SER & $\mathrm{HA}$ & 18.062 \\
\hline 66 & THR & $\mathrm{CA}$ & 66 & THR & $\mathrm{HA}$ & 10.475 \\
\hline 67 & LEU & $\mathrm{CA}$ & 67 & LEU & $\mathrm{HA}$ & 6.716 \\
\hline 69 & LEU & $\mathrm{CA}$ & 69 & LEU & $\mathrm{HA}$ & 5.977 \\
\hline 70 & VAL & $\mathrm{CA}$ & 70 & VAL & $\mathrm{HA}$ & 11.118 \\
\hline 71 & LEU & $\mathrm{CA}$ & 71 & LEU & $\mathrm{HA}$ & 14.458 \\
\hline 72 & $\mathrm{ARG}$ & $\mathrm{CA}$ & 72 & $\mathrm{ARG}$ & $\mathrm{HA}$ & 13.446 \\
\hline 73 & LEU & $\mathrm{CA}$ & 73 & LEU & $\mathrm{HA}$ & 6.784 \\
\hline 74 & ARG & $\mathrm{CA}$ & 74 & ARG & HA & 7.838 \\
\hline 75 & GLY & $\mathrm{CA}$ & 75 & GLY & $\mathrm{HA}$ & 10.529 \\
\hline
\end{tabular}

Table B.5: Residual dipolar couplings measured for citrate bound form of CitAP in phages

\begin{tabular}{|c|c|c|c|c|c|c|}
\hline Res: Number1 & Res: name1 & Atom1 & Res: Number2 & Res: name2 & Atom2 & $\mathbf{D}$ \\
\hline 3 & ILE & $\mathrm{H}$ & 3 & ILE & $\mathrm{N}$ & 0.584 \\
\hline 4 & THR & $\mathrm{H}$ & 4 & THR & $\mathrm{N}$ & -1.763 \\
\hline 6 & GLU & $\mathrm{H}$ & 6 & GLU & $\mathrm{N}$ & 2.450 \\
\hline 7 & $\mathrm{ARG}$ & $\mathrm{H}$ & 7 & $\mathrm{ARG}$ & $\mathrm{N}$ & -0.618 \\
\hline
\end{tabular}


Table B.5 - continued from previous page

\begin{tabular}{|c|c|c|c|c|c|c|}
\hline Res: Number1 & Res: name1 & Atom1 & Res: Number2 & Res: name2 & Atom2 & $\mathbf{D}$ \\
\hline 9 & HIS & $\mathrm{H}$ & 9 & HIS & $\mathrm{N}$ & 3.238 \\
\hline 13 & GLY & $\mathrm{H}$ & 13 & GLY & $\mathrm{N}$ & 5.361 \\
\hline 14 & GLN & $\mathrm{H}$ & 14 & GLN & $\mathrm{N}$ & 1.070 \\
\hline 21 & MET & $\mathrm{H}$ & 21 & MET & $\mathrm{N}$ & 2.672 \\
\hline 25 & ALA & $\mathrm{H}$ & 25 & ALA & $\mathrm{N}$ & -0.422 \\
\hline 26 & MET & $\mathrm{H}$ & 26 & MET & $\mathrm{N}$ & -8.741 \\
\hline 29 & LEU & $\mathrm{H}$ & 29 & LEU & $\mathrm{N}$ & 6.818 \\
\hline 30 & VAL & $\mathrm{H}$ & 30 & VAL & $\mathrm{N}$ & 0.889 \\
\hline 31 & GLU & $\mathrm{H}$ & 31 & GLU & $\mathrm{N}$ & 0.361 \\
\hline 32 & ALA & $\mathrm{H}$ & 32 & ALA & $\mathrm{N}$ & 3.582 \\
\hline 33 & VAL & $\mathrm{H}$ & 33 & VAL & $\mathrm{N}$ & 4.160 \\
\hline 34 & GLN & $\mathrm{H}$ & 34 & GLN & $\mathrm{N}$ & 0.858 \\
\hline 35 & LYS & $\mathrm{H}$ & 35 & LYS & $\mathrm{N}$ & 0.562 \\
\hline 36 & ARG & $\mathrm{H}$ & 36 & ARG & $\mathrm{N}$ & 8.836 \\
\hline 37 & ASP & $\mathrm{H}$ & 37 & ASP & $\mathrm{N}$ & 3.856 \\
\hline 38 & LEU & $\mathrm{H}$ & 38 & LEU & $\mathrm{N}$ & 4.581 \\
\hline 39 & ALA & $\mathrm{H}$ & 39 & ALA & $\mathrm{N}$ & 2.103 \\
\hline 40 & ARG & $\mathrm{H}$ & 40 & ARG & $\mathrm{N}$ & -0.600 \\
\hline 41 & ILE & $\mathrm{H}$ & 41 & ILE & $\mathrm{N}$ & 2.867 \\
\hline 42 & LYS & $\mathrm{H}$ & 42 & LYS & $\mathrm{N}$ & 5.602 \\
\hline 43 & ALA & $\mathrm{H}$ & 43 & ALA & $\mathrm{N}$ & 0.696 \\
\hline 45 & ILE & $\mathrm{H}$ & 45 & ILE & $\mathrm{N}$ & 3.331 \\
\hline 56 & TYR & $\mathrm{H}$ & 56 & TYR & $\mathrm{N}$ & 7.518 \\
\hline 59 & VAL & $\mathrm{H}$ & 59 & VAL & $\mathrm{N}$ & 3.405 \\
\hline 60 & GLY & $\mathrm{H}$ & 60 & GLY & $\mathrm{N}$ & 4.796 \\
\hline 61 & ASP & $\mathrm{H}$ & 61 & ASP & $\mathrm{N}$ & -5.918 \\
\hline 62 & ALA & $\mathrm{H}$ & 62 & ALA & $\mathrm{N}$ & 3.451 \\
\hline 63 & SER & $\mathrm{H}$ & 63 & SER & $\mathrm{N}$ & 6.668 \\
\hline 64 & GLY & $\mathrm{H}$ & 64 & GLY & $\mathrm{N}$ & -5.512 \\
\hline 65 & GLN & $\mathrm{H}$ & 65 & GLN & $\mathrm{N}$ & -9.921 \\
\hline 66 & ARG & $\mathrm{H}$ & 66 & ARG & $\mathrm{N}$ & -6.680 \\
\hline 68 & TYR & $\mathrm{H}$ & 68 & TYR & $\mathrm{N}$ & 7.935 \\
\hline 69 & HIS & $\mathrm{H}$ & 69 & HIS & $\mathrm{N}$ & 9.256 \\
\hline 70 & VAL & $\mathrm{H}$ & 70 & VAL & $\mathrm{N}$ & 11.106 \\
\hline 73 & ASP & $\mathrm{H}$ & 73 & ASP & $\mathrm{N}$ & -3.705 \\
\hline 75 & ILE & $\mathrm{H}$ & 75 & ILE & $\mathrm{N}$ & 1.163 \\
\hline 76 & GLY & $\mathrm{H}$ & 76 & GLY & $\mathrm{N}$ & 0.704 \\
\hline 77 & LYS & $\mathrm{H}$ & 77 & LYS & $\mathrm{N}$ & -1.498 \\
\hline 78 & SER & $\mathrm{H}$ & 78 & SER & $\mathrm{N}$ & -2.658 \\
\hline 81 & GLY & $\mathrm{H}$ & 81 & GLY & $\mathrm{N}$ & -1.790 \\
\hline 83 & ASP & $\mathrm{H}$ & 83 & ASP & $\mathrm{N}$ & 2.420 \\
\hline 85 & ASP & $\mathrm{H}$ & 85 & ASP & $\mathrm{N}$ & -5.986 \\
\hline
\end{tabular}


Table B.5 - continued from previous page

\begin{tabular}{|c|c|c|c|c|c|c|}
\hline Res: Number1 & Res: name1 & Atom1 & Res: Number2 & Res: name2 & Atom2 & D \\
\hline 86 & GLU & $\mathrm{H}$ & 86 & GLU & $\mathrm{N}$ & -8.760 \\
\hline 87 & ALA & $\mathrm{H}$ & 87 & ALA & $\mathrm{N}$ & -10.412 \\
\hline 88 & LEU & $\mathrm{H}$ & 88 & LEU & $\mathrm{N}$ & -8.587 \\
\hline 92 & LYS & $\mathrm{H}$ & 92 & LYS & $\mathrm{N}$ & -1.988 \\
\hline 93 & SER & $\mathrm{H}$ & 93 & SER & $\mathrm{N}$ & 4.620 \\
\hline 94 & TYR & $\mathrm{H}$ & 94 & TYR & $\mathrm{N}$ & 10.087 \\
\hline 96 & SER & $\mathrm{H}$ & 96 & SER & $\mathrm{N}$ & 6.719 \\
\hline 109 & LYS & $\mathrm{H}$ & 109 & LYS & $\mathrm{N}$ & 11.118 \\
\hline 110 & SER & $\mathrm{H}$ & 110 & SER & $\mathrm{N}$ & 7.454 \\
\hline 114 & ASP & $\mathrm{H}$ & 114 & ASP & $\mathrm{N}$ & 0.392 \\
\hline 115 & ALA & $\mathrm{H}$ & 115 & ALA & $\mathrm{N}$ & 10.445 \\
\hline 117 & GLY & $\mathrm{H}$ & 117 & GLY & $\mathrm{N}$ & 3.891 \\
\hline 119 & VAL & $\mathrm{H}$ & 119 & VAL & $\mathrm{N}$ & 1.904 \\
\hline 120 & ILE & $\mathrm{H}$ & 120 & ILE & $\mathrm{N}$ & 1.010 \\
\hline 121 & GLY & $\mathrm{H}$ & 121 & GLY & $\mathrm{N}$ & 8.121 \\
\hline 122 & ILE & $\mathrm{H}$ & 122 & ILE & $\mathrm{N}$ & 6.566 \\
\hline 123 & VAL & $\mathrm{H}$ & 123 & VAL & $\mathrm{N}$ & 5.976 \\
\hline 127 & TYR & $\mathrm{H}$ & 127 & TYR & $\mathrm{N}$ & 4.012 \\
\hline 128 & THR & $\mathrm{H}$ & 128 & THR & $\mathrm{N}$ & 5.460 \\
\hline 129 & ILE & $\mathrm{H}$ & 129 & ILE & $\mathrm{N}$ & -0.205 \\
\hline 130 & GLU & $\mathrm{H}$ & 130 & GLU & $\mathrm{N}$ & 2.096 \\
\hline 131 & GLN & $\mathrm{H}$ & 131 & GLN & $\mathrm{N}$ & -0.308 \\
\hline 132 & LEU & $\mathrm{H}$ & 132 & LEU & $\mathrm{N}$ & 0.361 \\
\hline 133 & GLU & $\mathrm{H}$ & 133 & GLU & $\mathrm{N}$ & 2.116 \\
\hline
\end{tabular}

Table B.6: Residual dipolar couplings measured for citrate free form of CitAP in phages

\begin{tabular}{|c|c|c|c|c|c|c|}
\hline Res: Number1 & Res: name1 & Atom1 & Res: Number2 & Res: name2 & Atom2 & D \\
\hline 18 & ILE & $\mathrm{H}$ & 18 & ILE & $\mathrm{N}$ & -10.411 \\
\hline 22 & GLN & $\mathrm{H}$ & 22 & GLN & $\mathrm{N}$ & -12.835 \\
\hline 24 & SER & $\mathrm{H}$ & 24 & SER & $\mathrm{N}$ & 2.122 \\
\hline 25 & ALA & $\mathrm{H}$ & 25 & ALA & $\mathrm{N}$ & -9.721 \\
\hline 26 & MET & $\mathrm{H}$ & 26 & MET & $\mathrm{N}$ & -15.662 \\
\hline 28 & GLU & $\mathrm{H}$ & 28 & GLU & $\mathrm{N}$ & 0.839 \\
\hline 29 & LEU & $\mathrm{H}$ & 29 & LEU & $\mathrm{N}$ & 11.105 \\
\hline 30 & VAL & $\mathrm{H}$ & 30 & VAL & $\mathrm{N}$ & 0.391 \\
\hline 31 & GLU & $\mathrm{H}$ & 31 & GLU & $\mathrm{N}$ & 0.997 \\
\hline 32 & ALA & $\mathrm{H}$ & 32 & ALA & $\mathrm{N}$ & 4.118 \\
\hline 33 & VAL & $\mathrm{H}$ & 33 & VAL & $\mathrm{N}$ & 7.505 \\
\hline 34 & GLN & $\mathrm{H}$ & 34 & GLN & $\mathrm{N}$ & 0.602 \\
\hline
\end{tabular}


Table B.6 - continued from previous page

\begin{tabular}{|c|c|c|c|c|c|c|}
\hline Res: Number1 & Res: name1 & Atom1 & Res: Number2 & Res: name2 & Atom2 & D \\
\hline 35 & LYS & $\mathrm{H}$ & 35 & LYS & $\mathrm{N}$ & 2.608 \\
\hline 37 & ASP & $\mathrm{H}$ & 37 & ASP & $\mathrm{N}$ & 11.477 \\
\hline 38 & LEU & $\mathrm{H}$ & 38 & LEU & $\mathrm{N}$ & 6.496 \\
\hline 39 & ALA & $\mathrm{H}$ & 39 & ALA & $\mathrm{N}$ & -4.833 \\
\hline 40 & ARG & $\mathrm{H}$ & 40 & ARG & $\mathrm{N}$ & -1.974 \\
\hline 41 & ILE & $\mathrm{H}$ & 41 & ILE & $\mathrm{N}$ & -1.458 \\
\hline 42 & LYS & $\mathrm{H}$ & 42 & LYS & $\mathrm{N}$ & -2.527 \\
\hline 43 & ALA & $\mathrm{H}$ & 43 & ALA & $\mathrm{N}$ & 0.884 \\
\hline 44 & LEU & $\mathrm{H}$ & 44 & LEU & $\mathrm{N}$ & -3.026 \\
\hline 61 & ASP & $\mathrm{H}$ & 61 & ASP & $\mathrm{N}$ & -5.673 \\
\hline 63 & SER & $\mathrm{H}$ & 63 & SER & $\mathrm{N}$ & 15.812 \\
\hline 112 & ILE & $\mathrm{H}$ & 112 & ILE & $\mathrm{N}$ & -2.552 \\
\hline 114 & ASP & $\mathrm{H}$ & 114 & ASP & $\mathrm{N}$ & 1.370 \\
\hline 115 & ALA & $\mathrm{H}$ & 115 & ALA & $\mathrm{N}$ & 18.573 \\
\hline 117 & GLY & $\mathrm{H}$ & 117 & GLY & $\mathrm{N}$ & 7.779 \\
\hline 118 & LYS & $\mathrm{H}$ & 118 & LYS & $\mathrm{N}$ & -2.091 \\
\hline 119 & VAL & $\mathrm{H}$ & 119 & VAL & $\mathrm{N}$ & 9.551 \\
\hline 120 & ILE & $\mathrm{H}$ & 120 & ILE & $\mathrm{N}$ & -3.127 \\
\hline 121 & GLY & $\mathrm{H}$ & 121 & GLY & $\mathrm{N}$ & 13.372 \\
\hline 122 & ILE & $\mathrm{H}$ & 122 & ILE & $\mathrm{N}$ & 16.623 \\
\hline 131 & GLN & $\mathrm{H}$ & 131 & GLN & $\mathrm{N}$ & 2.057 \\
\hline 132 & LEU & $\mathrm{H}$ & 132 & LEU & $\mathrm{N}$ & 3.035 \\
\hline 17 & LEU & $\mathrm{C}$ & 18 & ILE & $\mathrm{N}$ & -0.889 \\
\hline 19 & GLN & $\mathrm{C}$ & 20 & ALA & $\mathrm{N}$ & -0.371 \\
\hline 21 & MET & $\mathrm{C}$ & 22 & GLN & $\mathrm{N}$ & -0.131 \\
\hline 23 & ILE & $\mathrm{C}$ & 24 & SER & $\mathrm{N}$ & 0.710 \\
\hline 24 & SER & $\mathrm{C}$ & 25 & ALA & $\mathrm{N}$ & -0.243 \\
\hline 25 & ALA & $\mathrm{C}$ & 26 & MET & $\mathrm{N}$ & 0.508 \\
\hline 27 & PRO & $\mathrm{C}$ & 28 & GLU & $\mathrm{N}$ & 0.081 \\
\hline 28 & GLU & $\mathrm{C}$ & 29 & LEU & $\mathrm{N}$ & 0.129 \\
\hline 29 & LEU & $\mathrm{C}$ & 30 & VAL & $\mathrm{N}$ & 0.128 \\
\hline 30 & VAL & $\mathrm{C}$ & 31 & GLU & $\mathrm{N}$ & 0.367 \\
\hline 31 & GLU & $\mathrm{C}$ & 32 & ALA & $\mathrm{N}$ & -1.570 \\
\hline 32 & ALA & $\mathrm{C}$ & 33 & VAL & $\mathrm{N}$ & 1.899 \\
\hline 33 & VAL & $\mathrm{C}$ & 34 & GLN & $\mathrm{N}$ & -1.769 \\
\hline 34 & GLN & $\mathrm{C}$ & 35 & LYS & $\mathrm{N}$ & 0.755 \\
\hline 35 & LYS & $\mathrm{C}$ & 36 & ARG & $\mathrm{N}$ & -0.275 \\
\hline 36 & ARG & $\mathrm{C}$ & 37 & ASP & $\mathrm{N}$ & -0.426 \\
\hline 37 & ASP & $\mathrm{C}$ & 38 & LEU & $\mathrm{N}$ & -1.125 \\
\hline 38 & LEU & $\mathrm{C}$ & 39 & ALA & $\mathrm{N}$ & 1.612 \\
\hline 39 & ALA & $\mathrm{C}$ & 40 & ARG & $\mathrm{N}$ & -1.189 \\
\hline 40 & ARG & $\mathrm{C}$ & 41 & ILE & $\mathrm{N}$ & 0.242 \\
\hline
\end{tabular}


Table B.6 - continued from previous page

\begin{tabular}{|c|c|c|c|c|c|c|}
\hline Res: Number1 & Res: name1 & Atom1 & Res: Number2 & Res: name2 & Atom2 & D \\
\hline 41 & ILE & C & 42 & LYS & N & 0.262 \\
42 & LYS & C & 43 & ALA & N & -0.825 \\
43 & ALA & C & 44 & LEU & N & 0.941 \\
60 & GLY & C & 61 & ASP & N & 0.495 \\
61 & ASP & C & 62 & ALA & N & -1.803 \\
62 & ALA & C & 63 & SER & N & 1.150 \\
113 & GLN & C & 114 & ASP & N & -0.477 \\
114 & ASP & C & 115 & ALA & N & -0.430 \\
116 & THR & C & 117 & GLY & N & -1.592 \\
117 & GLY & C & 118 & LYS & N & 1.231 \\
118 & LYS & C & 119 & VAL & N & -1.306 \\
119 & VAL & C & 120 & ILE & N & 0.204 \\
120 & ILE & C & 121 & GLY & N & -0.069 \\
132 & LEU & C & 133 & GLU & N & -0.170 \\
\hline \hline
\end{tabular}




\section{Appendix C}

\section{Bruker Pulseprogramme and MATHEMATICA scripts}

Bruker pulse-programme for $\mathrm{CBCA}(\mathrm{CO}) \mathrm{NH}$ experiment used for simultaneous measurement of ${ }^{1} \mathbf{J}_{C \alpha H \alpha},{ }^{1} \mathbf{J}_{C \beta H \beta}$ and ${ }^{1} \mathbf{J}_{C \alpha C^{\prime}}$ couplings:

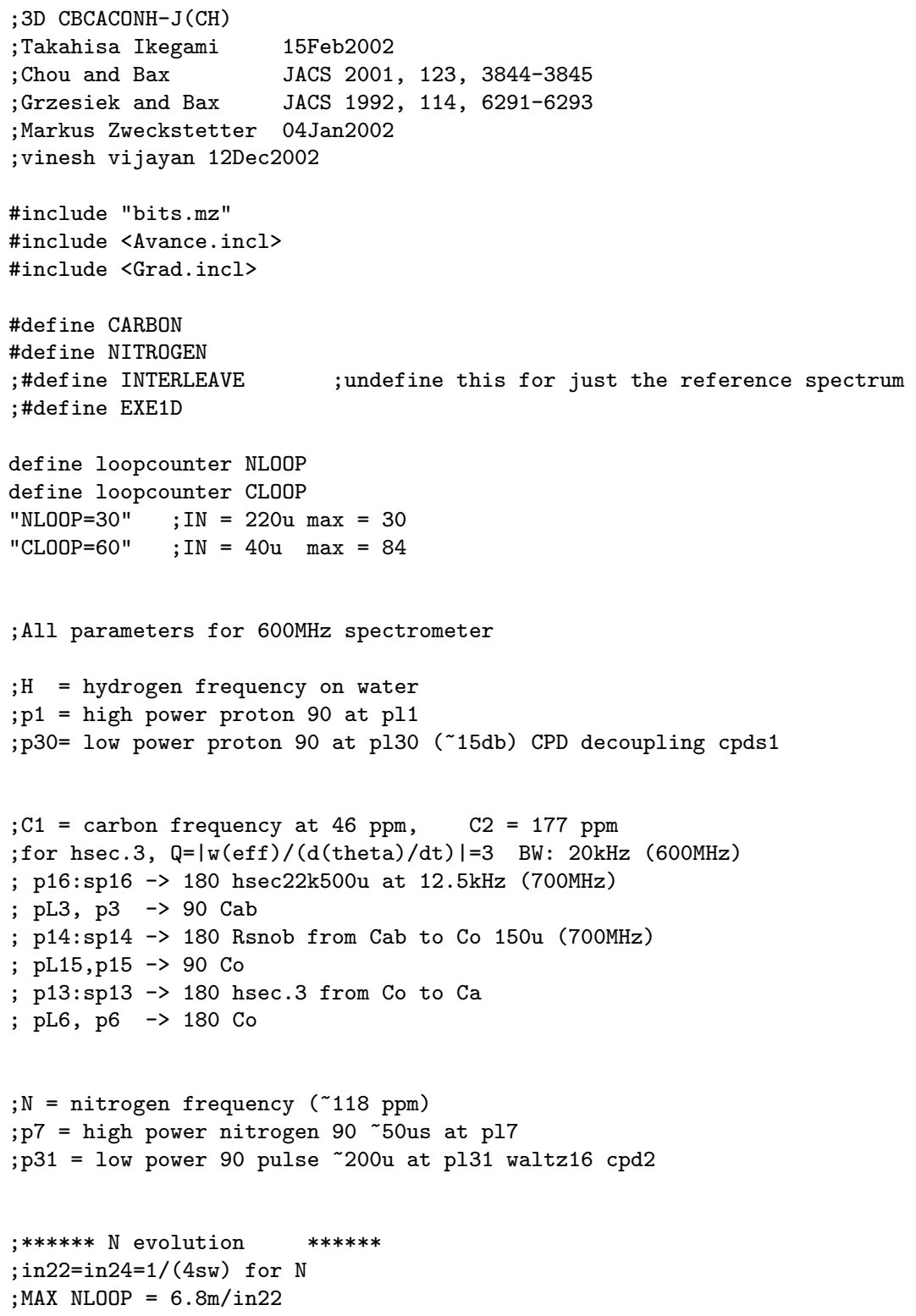




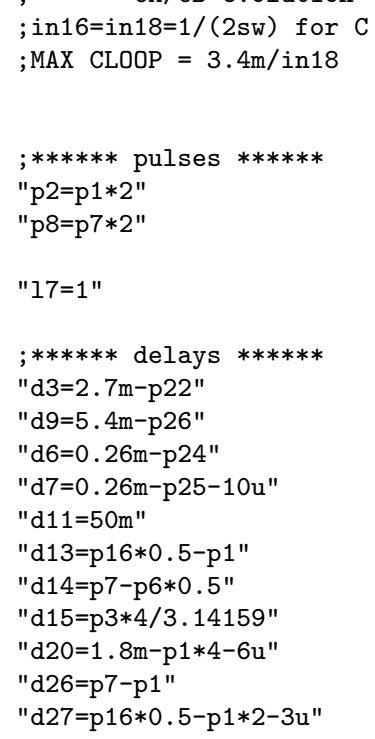


300u UNBLKGRAD

(p1 ph7):H

$10 \mathrm{u}$

2mp: gp1

$100 \mathrm{u}$

300u pl3: C1

;******* inept to $\mathrm{C}$ aliphatic $* * * * * * *$

(p3 ph4):C1

(p16:sp2 ph10):C1 (d27 p1 ph10 3u p2 ph11 3u p1 ph10):H

d15 $; \mathrm{p} 3 * 4 / \mathrm{Pi}$

d16 ;d16 increment delay

(p14:sp14 ph10):C1 ;sinc1.0 C' refocusing ( $150 \mathrm{u})$

$100 \mathrm{u}$

if " $17==1$ or 4 " goto $71 ; 1.93 \mathrm{~m}$ length of effective J-modulation

if "17==2" goto $72 \quad ; 3.73 \mathrm{~m} \quad \ldots$

71

$; 7.22 \mathrm{~m} \quad$.

$71600 u$

(p1 ph10 3u p2 ph11 3u p1 ph10):H

d20

$900 \mathrm{u}$

goto 74

$72 \quad 1.5 \mathrm{~m}$

(p1 ph10 3u p2 ph11 3u p1 ph10):H

d20

goto 74

$73 \quad 1.5 \mathrm{~m}$

d20

(p1 ph10 3u p2 ph11 3u p1 ph10):H

$74 \quad 5 u$

20u pl30:H

(p16:sp2 ph17):C1

d18

; d18 decrement delay

(p14:sp14 ph10):C1

;B.S. compensate

20u pl3: C1

(p3 ph10):C1

;******* inept to ca

$5 \mathrm{u}$

20u cpds $1: \mathrm{H}$

(p14:sp14 ph10):C1

; sinc compensation

(p16:sp2 ph10):C1

30u

d15

$3.4 \mathrm{~m}$

(p14:sp14 ph10):C1

$5 \mathrm{u}$

(p16:sp2 ph10):C1

$5 \mathrm{u}$

400u p13:C

(p3 ph10):C1

$5 \mathrm{u}$

20u do: $\mathrm{H}$

1. 4mp:gp2

$100 u$

100u fq2: C1

100u pl30:H

80u pl15:C1

20u cpds $1: \mathrm{H}$

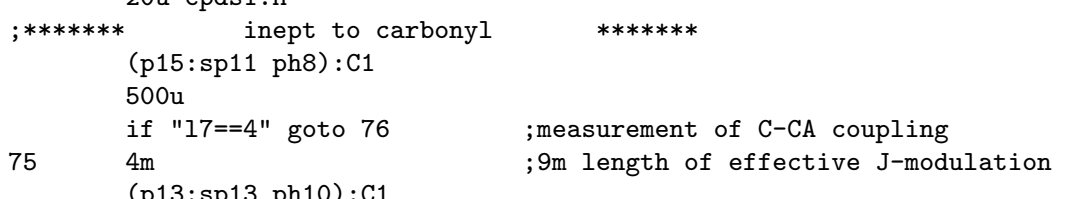

; hsec.3 compensation

; sinc on $\mathrm{C}^{\prime}$

; 500 u hsec. 3 on $\mathrm{Ca}$

$;->$ Co

;9m length of effective J-modulation 


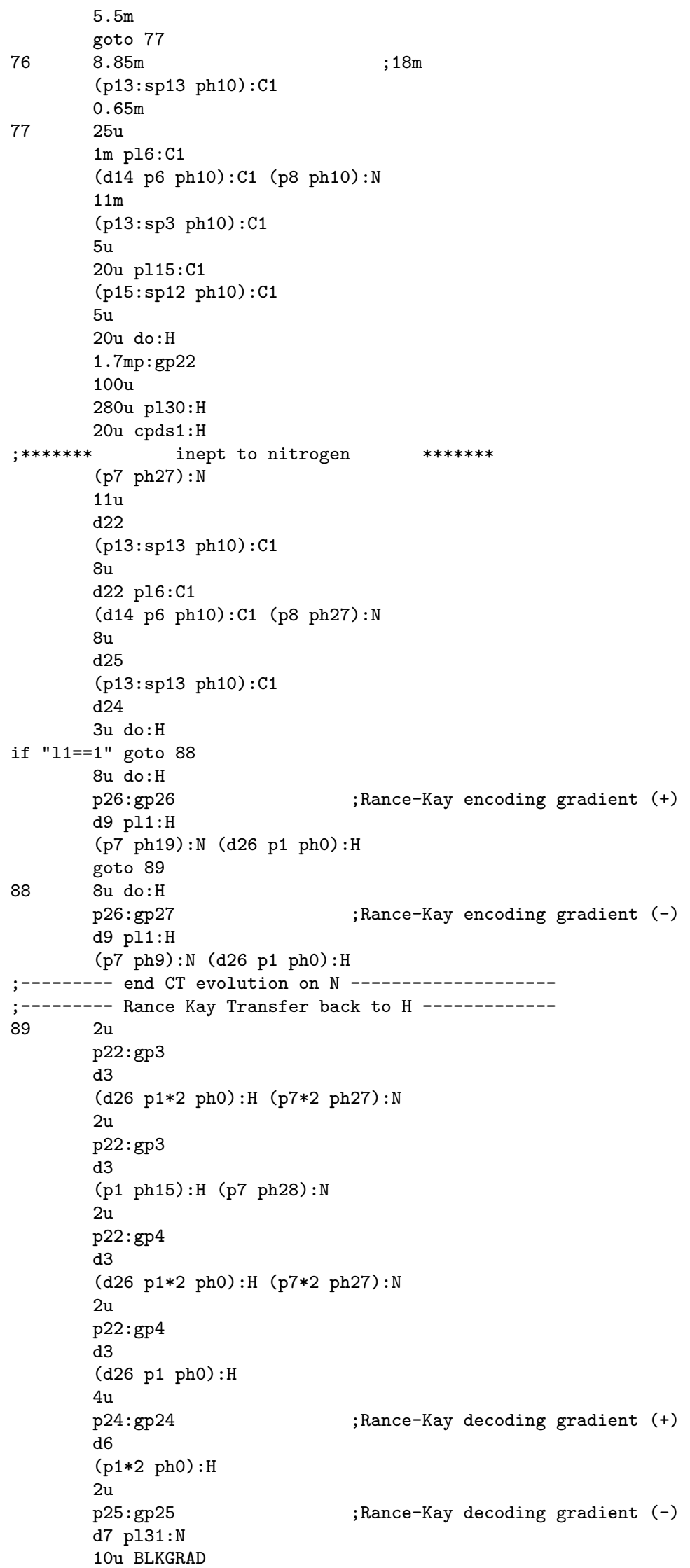




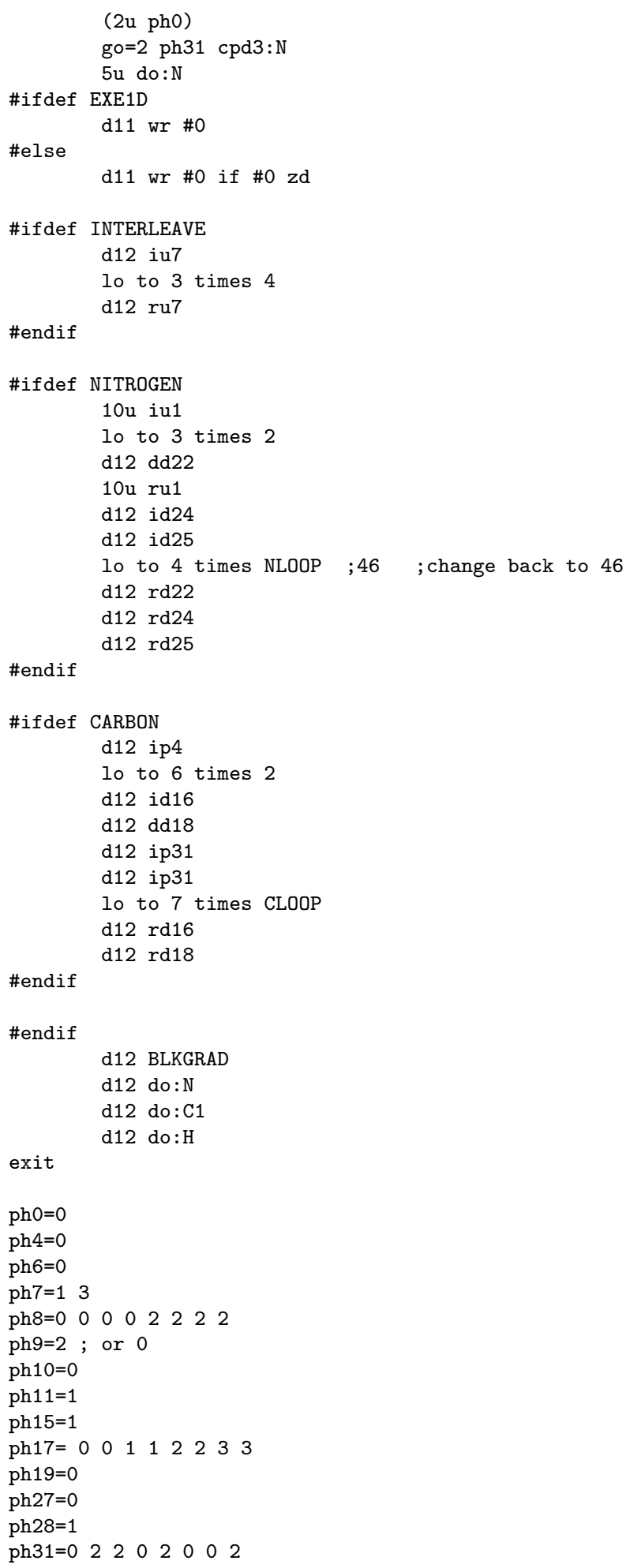

Bruker pulse-programme for TROSY-HNCO experiment for simultaneous measurement of ${ }^{1} \mathbf{J}_{C^{\prime} N}$ and ${ }^{1} \mathbf{J}_{N H}$ couplings. 
\#include <Avance.incl>

\#include <Grad.incl>

;3D TROSY-HNCOJ

; Modified from the original pulse scheme by vivi, for including shorter NCo transfer delay for the 3rd interleaved experiment. ;3D TROSY-HNCOJ

; NOTE: C' on C1; CA on C1 using shaped pulses with offset

; ----------------------

;--RF Pulses---------------

$; \mathrm{p} 1=90 \mathrm{deg}$ (10us) $1 \mathrm{H}$ pulse Qpl1

$; \mathrm{p} 2=90 \mathrm{deg}$ @pl2 1H; should be $\sim 1 \mathrm{~ms}$

$; \mathrm{p} 3=90$ hard on $C^{\prime}$

;p6 $=$ sp0 90 sinc1.0 on $C^{\prime}(\sim 100$ us at $800 \mathrm{MHz})$, center lobe $\operatorname{sinx} / \mathrm{x}$

$; \mathrm{p} 7=90 \mathrm{deg}(\sim 50 \mathrm{us}) 15 \mathrm{~N}$ pulse @pl7

; 8 8 $=$ sp3 sinc1.0 on $\mathrm{CA}(\sim 100$ us at $800 \mathrm{MHz})$

;p16= sp2 $180 \mathrm{hsec} .3$ on C' (1000 us at $800 \mathrm{MHz})$, hyperbolic secant

;--Gradients---------------

$\begin{array}{ll}; p 21=1 \mathrm{~m}, \text { gp1 }=5 \% \mathrm{x} & \text { sine. } 50 \\ ; p 20=1 \mathrm{~m}, \text { gp0 }=30 \% \mathrm{y} & \text { sine. } 50 \\ ; p 20=1 \mathrm{~m}, \text { gp2 }=30 \% \mathrm{x} & \text { sine. } 50 \\ ; p 21=1 \mathrm{~m}, \text { gp3 }=30 \% \mathrm{z} & \text { sine. } 50\end{array}$

;--Options----------------

;--Options-

\#define NITRO

\#define CARBON

\#define INTERLEAVE

;--Evolutions--

define loopcounter NLOOP

define loopcounter CLOOP

"NLOOP=36"

; ; ; "CLOOP=32"

$; \max =62$ for $500 \mathrm{u}$ increment in $15 \mathrm{~N}$ dim

"CLOOP=18"

; sine bell shaped gradient

;number of complex C' increment

$; 15 \mathrm{~N}$ evolution in20=in21=in22=in24 Total evolution $=4 *$ in $20 *$ NLOOP

;C' evolution ino Total evolution $=2 *$ in $0 *$ CLOOP

; --Delays

"d0=in $0 * 0.5-p 8 * 0.5-p 6 * 0.54 "$

"d3=2.5m-p21"

;90,-180 phase correction in C' dim

"d4=2.6m-p21-p2"

; "d5=16.6m-p2-40u"

;"d6=33.1m-p2-40u"

"d7=d3-p2-20u"

"d11=50m"

"d16=p16"

"d19=in $21 * 0.444 "$

"d20=7.5m+p16*0.5"

;fudge delay for zero ph1 in $15 \mathrm{~N}$ dim

"d21=7.5m"

"d22=7.5m"

"d23=d20-p16-5u"

"d24=2u"

"d26=p7-p1"

"d27=p16*0.5-p7"

"d28=d24*2"

"d29=p8*0.5-p7"

1 


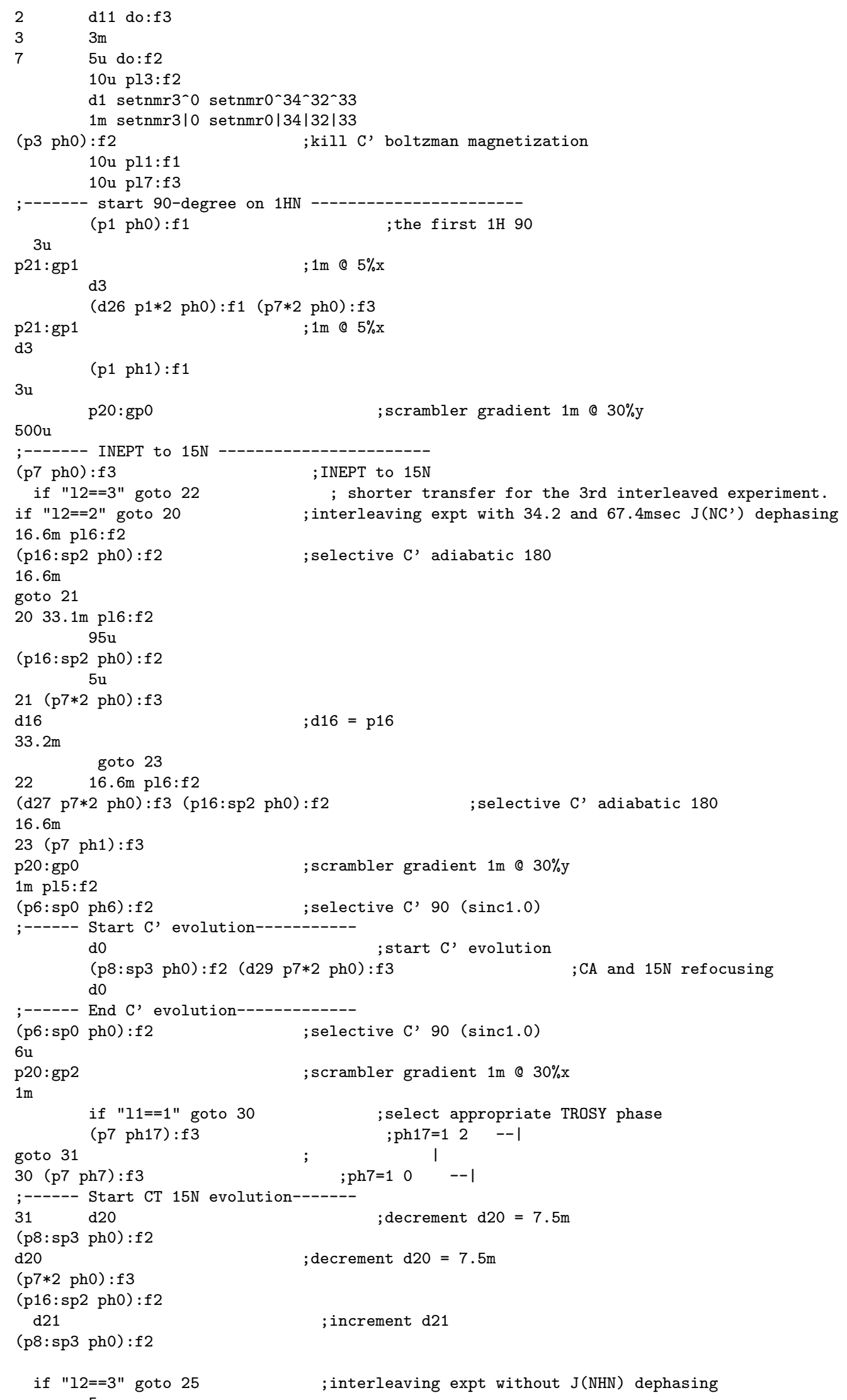




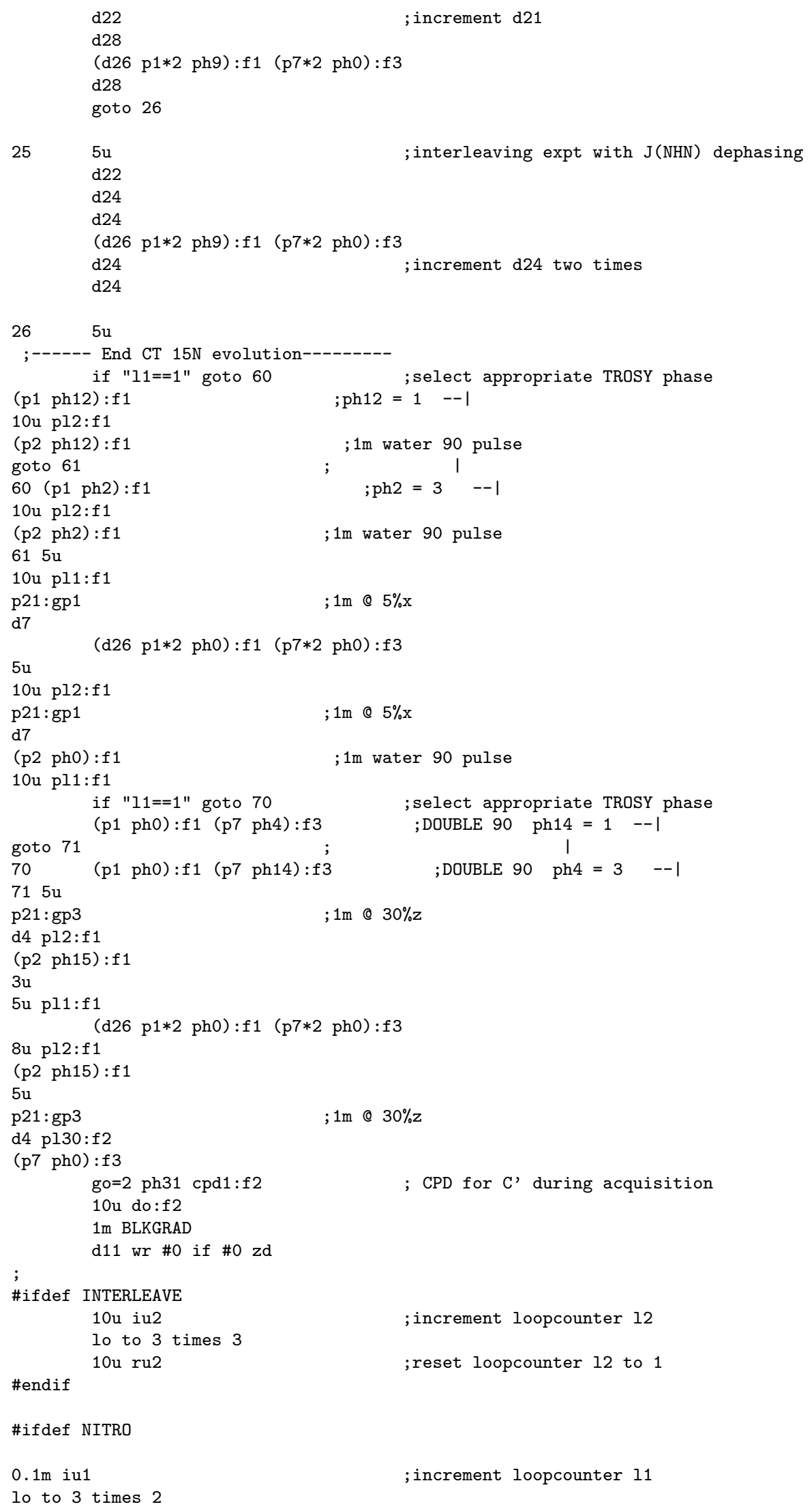




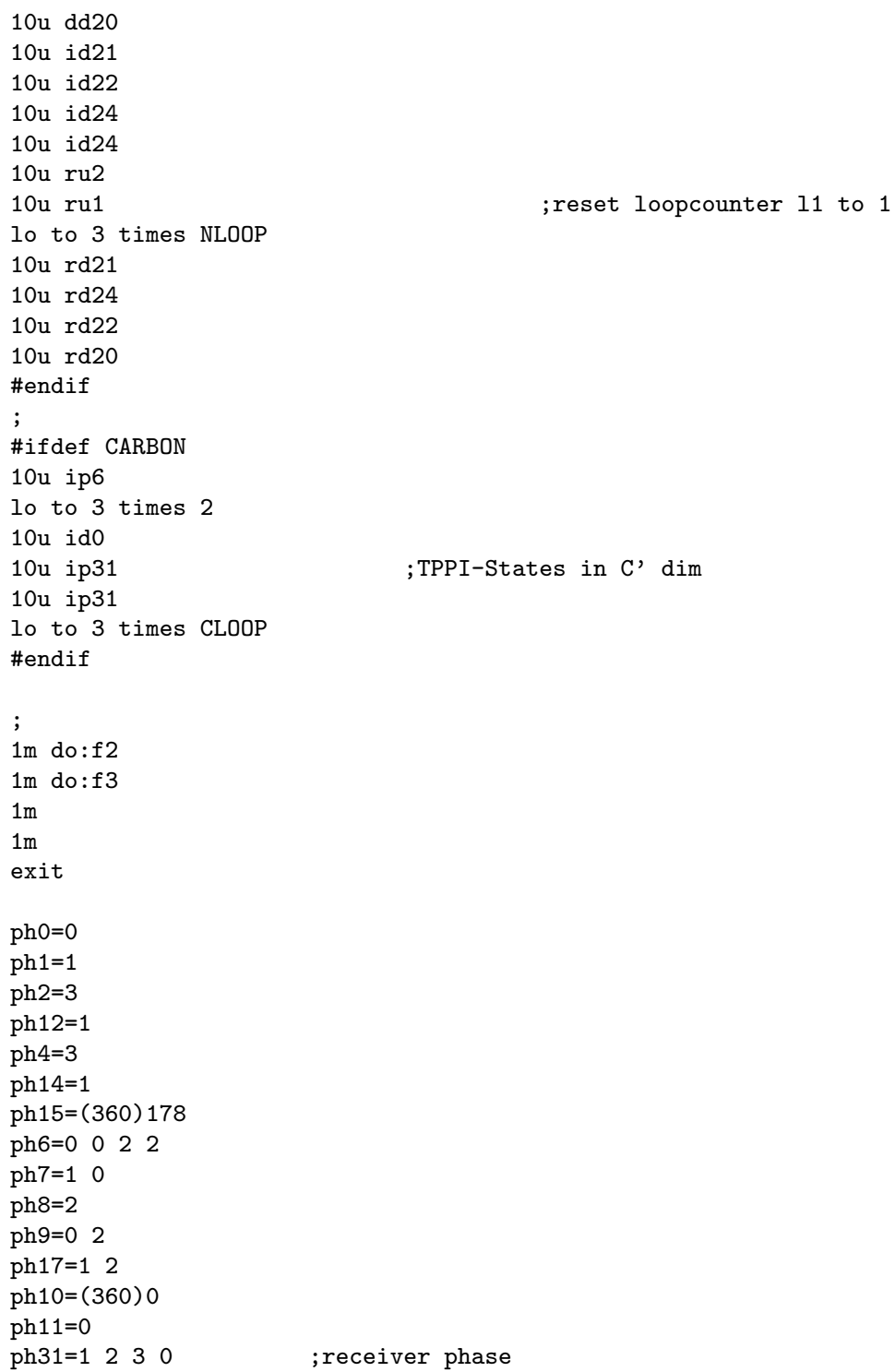

\section{C.1 MATHEMATICA scripts}

MATHEMATICA scripts for fitting RDCs to a structure.

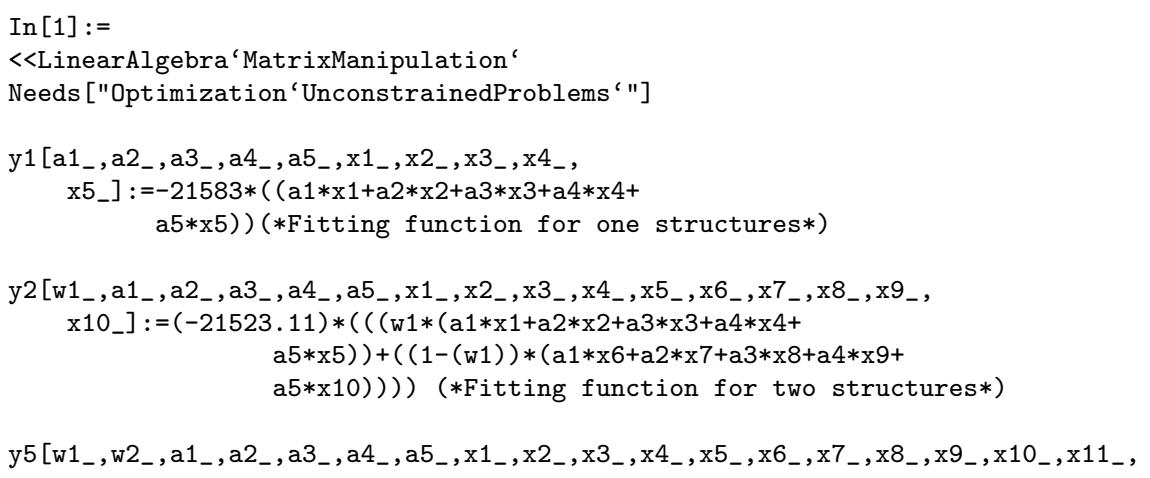




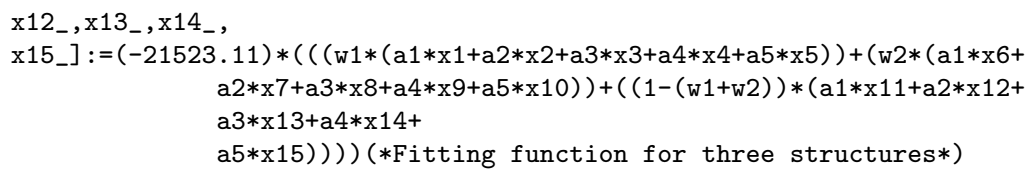

$(* * * * * * * * * * * * * * * * * * * * * * * 5 * * * * * * * * * * * * * * * * * * * * * * * * * * * * * * * * * * *))$

(*an example of fitting procedure for three structures*)

erfunc5 [w1 $1_{-}, \mathrm{w} 2_{-}, \mathrm{a} 1_{-}, \mathrm{a} 2_{-}, \mathrm{a} 3_{-}, \mathrm{a} 4_{-}, \mathrm{a} 5_{-}$,

datum_] : = (y5[w1, w2 $, \mathrm{a} 1, \mathrm{a} 2, \mathrm{a} 3, \mathrm{a} 4, \mathrm{a} 5, \operatorname{datum}[[1]], \operatorname{datum}[[2]], \operatorname{datum}[[3]]$, $\operatorname{datum}[[4]], \operatorname{datum}[[5]]$, datum $[[6]]$, datum $[[7]], \operatorname{datum}[[8]]$, datum $[[9]]$, $\operatorname{datum}[[10]], \operatorname{datum}[[11]], \operatorname{datum}[[12]], \operatorname{datum}[[13]], \operatorname{datum}[[14]]$, datum $[[15]]]-\operatorname{datum}[[16]])^{\wedge} 2$ (*error function for three structures*)

$\operatorname{In}[30]:=$

chisq5=Map [erfunc5 [w1, w2, a1, a2, a3, a4, a5,\#]\&, data ] ;

soln5=NMinimize [\{Apply [Plus, chisq5], 1>w1>0\&\&1>w2>0\}, \{w1, w2, a1, a2, a3, a4, a5\}] (*fitting function for three structures*)

Out $[31]=$

$\{534.878,\{\mathrm{a} 1->-0.000344807, \mathrm{a} 2->0.000159985, \mathrm{a3}->]-0.000718622$,

$\mathrm{a} 4->0.000152141, \mathrm{a} 5->]-0.000126468, \mathrm{w} 1->0.184418, \mathrm{w} 2->0\}$.

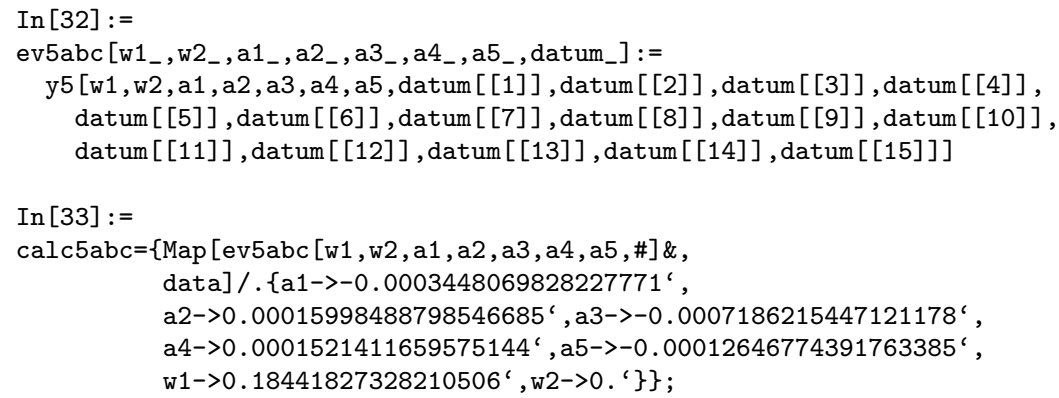

R5abc=Correlation $[\mathrm{Flatten}[\mathrm{ex}]$,

Flatten[calc5abc]] (*correlation function for three structures*)

Out $[34]=$

0.936602

Sqrt [Apply [Plus, (Flatten [ex]-Flatten [calc5abc]) ^2]/64]/

Sqrt [Apply[Plus,Flatten[ex] 2]/64] (*quality factor for three structures*)

Out $[35]=$

0.348577

MATHEMATICA scripts for fitting intensity ratios to obtain $\tau(\mathbf{c})$

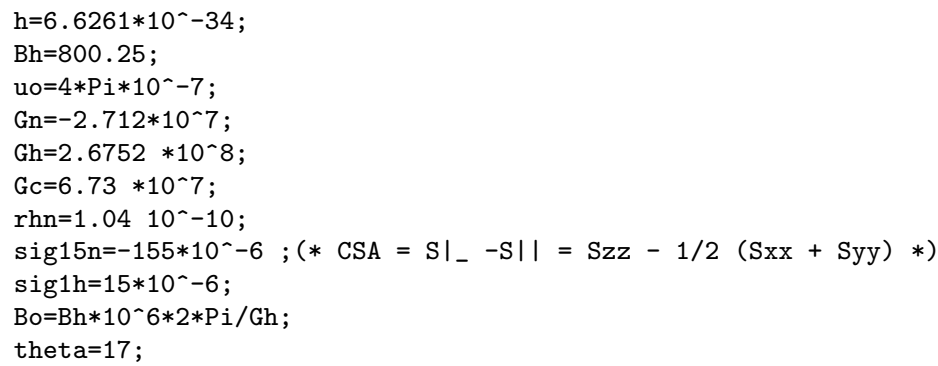


$<$ Statistics 'NonlinearFit'

$\mathrm{p}=(\mathrm{uo} * \mathrm{Gn} * \mathrm{Gh} * \mathrm{~h}) /\left(16 * \mathrm{Pi}^{\wedge} 2 * 2^{\wedge}(0.5) * \mathrm{rhn} ` 3\right)$

-24048 .

$\mathrm{d}=(\mathrm{Gn} * \mathrm{Bo} * \operatorname{sig} 15 \mathrm{n}) /\left(3 * 2^{\sim}(0.5)\right)$

18622.3

eta $=-2 * \mathrm{p} * \mathrm{~d} *((3 \operatorname{Cos}[$ theta* $\mathrm{Pi} / 180.] \wedge 2-1) / 2) * 4 * 0.4$

$1.2493077844773753 * 10^{\wedge} 9$

f3=Import ["C: \Vinesh \Desktop \Data1.dat", "Table"] ;

r1=Take [Flatten $[\mathrm{f} 3],\{2,200,4\}]$;

t1 $=$ PadLeft $\left[\{\}, 50,\left\{32 * 10^{-}-3\right\}\right]$;

11=Transpose [Partition [Join [t1,r1] , 50]] ;

r2=Take $[$ Flatten $[f 3],\{4,200,4\}]$;

id=Take $[F l a t t e n[f 3],\{1,200,4\}]$;

12=Transpose [Partition [Join [t2,r2] , 50]] ;

data1=Transpose [Partition [Join $[11,12], 50]]$;

For $[i=1, i \backslash[$ LessEqual] Part [Dimensions [data1], 1$], i=i+1, d x=\operatorname{Part}$ [data1, $i]$; $\mathrm{dy}=\operatorname{Part}[\mathrm{dx}, 2]-\operatorname{Part}[\mathrm{dx}, 1] ; \mathrm{Tc}=\operatorname{ArcTanh}[\operatorname{Part}[\mathrm{dy}, 2]] /(\operatorname{eta} * \operatorname{Part}[\mathrm{dy}, 1] * 0.8)$; Print [Tc] ]

$(* * * * * * * * * * * * * * * \quad * * * * * * * * * * * * * * * * * * * * * * * * * * * * * * * * *)$ 



\section{Lebenslauf}

\author{
Name \\ Geburtsdatum \\ Geburtsort \\ Staatsangehörigkeit \\ Religion \\ Familienstand \\ Vinesh Vijayan \\ 16.Dezember 1977 \\ Vaikom,Kerala (Indien) \\ Malayalam (Indien) \\ Hindu \\ Verheiratet
}

\section{Ausbildung}

1994-1996

1996-1999

1996-1999

2000-2002

2001

2002

2002

2002

2002-2007

2002-2007 Indien tayam, Indien dungsbehörde N. Chandrakumar dungsbehörde

Master in Chemie nungstest qualifiziert (Prof.Griesinger) in Göttingen

Gymnasium aus der Mahatma Gandhi Universität in Kottayam,

Bachelor in Chemie an der Mahatma Gandhi Universität in Kot-

Stipendium für studentische Exzellenz der staatlichen Bil-

Anfertigung einer Master-Arbeit am Indien Institut für Technologie Madras in Chennai, Indien

Thema der Master-Arbeit: "Selective Heteronuclear CrossPolarization in Scalar Coupled Spin Systems"; Betreuer: Prof. Dr.

Stipendium für studentische Exzellenz der staatlichen Bil-

Rat der wissenschaftlichen Industrieforschung - Nationaler Eig-

Graduierte Eignungsprüfung in der Technik qualifiziert

Wissenschaftlicher Mitarbeiter am Institut für Biophysikalische Chemie, Abteilung NMR basierte Strukturbiologie

Anfertigung der vorliegenden Doktorarbeit unter Prof. Dr. Christian Griesinger;

Thema der Doktorarbeit: "NMR Structural Studies on the Periplasmic Domain of CitA and DcuS".

Göttingen, den 21.03.2007 